\title{
MONODROMY OF HYPERGEOMETRIC FUNCTIONS AND NON-LATTICE INTEGRAL MONODROMY
}

\author{
by P. DELIGNE and G. D. MOSTOW (*)
}

\section{r. Introduction}

The hypergeometric series

$$
\mathrm{F}(a, b ; c ; x)=\sum_{n=0} \frac{(a, n)(b, n)}{(c, n)} \frac{x^{n}}{n !}, \quad|x|<\mathrm{I}
$$

where $(a, n)=\prod_{i=0}^{n-1}(a+i)$, defined for $c$ not an integer $\leq 0$, was first introduced by Euler in $177^{8}$ as a solution of the hypergeometric differential equation

$$
x(\mathrm{I}-x) y^{\prime \prime}+(c-(a+b+\mathrm{I}) x) y^{\prime}-a b y=0 .
$$

$\mathrm{F}(a, b ; c ; x)$ represents the unique solution of (2) which is holomorphic at $x=0$ and takes the value $\mathrm{I}$ at $x=0$. If neither $b$ nor $c-b$ is an integer $\leq 0$, Euler knew the integral representation

$$
\frac{\Gamma(b) \Gamma(c-b)}{\Gamma(c)} \mathrm{F}(a, b ; c ; x)=\int_{0}^{1} z^{b-1}(1-z)^{c-b-1}(\mathrm{I}-z x)^{-a} d z .
$$

Replacing $z$ by $u^{-1}$, (3) also has the form

$$
\int_{1}^{\infty} u^{a-c}(u-1)^{c-b-1}(u-x)^{-a} d u .
$$

If we integrate instead from $g$ to $h$ with $g$ and $h$ in $\{0, \mathrm{I}, \infty, x\}$ we get other solutions of (2), a fact discovered independently by Hermite [9], Pochhammer [ 17 ], and Schäfii [19]. Even when the integral diverges, it yields solutions of (2) when taken as its Hadamard " finite part", provided that the integrand does not have a pole of integral order at $g$ or $h$.

The hypergeometric equation (2) is the unique second order linear differential equation with regular singularities, singular only at $0,1, \infty$ with exponents $(0,1-c)$, $(0, c-a-b),(a, b)$ respectively. "Exponents $\left(\alpha, \alpha^{\prime}\right)$ at a singularity $p$ " means that suitable linearly independent linear combinations of the branches of a solution have the

(*) Supported in part by NSF Grant MCS-8203604. 
form $(x-p)^{\alpha} f_{1}(x), \quad(x-p)^{\alpha^{\prime}} f_{2}(x)$ with $f_{i}$ holomorphic around $p$; if $\alpha-\alpha^{\prime} \in \mathbf{Z}$ an additional logarithmic term is allowed. In 1857 , Riemann proved that the solutions of (2) are the only multivalued functions with exactly two linearly independent branches, branching only at $\mathrm{o}, \mathrm{I}, \infty$ with exponents as above (cf. [18]). Riemann's proof assumed that none of the exponent differences at a singularity was an integer and proceeded by first computing global monodromy. The above characterization of the hypergeometric equation is the basis of Fuchs' proof of Riemann's theorem (cf. [8]).

In his seminal paper [20], which seeks to determine the values $a, b, c$ for which the hypergeometric function is an algebraic function of $x$, Schwarz considered the map $x \mapsto w_{2}(x) / w_{1}(x)$ where $w_{1}$ and $w_{2}$ form a base of $\mathrm{W}$, the two dimensional linear space of all solutions of (2). Let $\hat{Q}$ denote the universal covering space of $\mathrm{Q}:=\mathbf{P}^{1}(\mathbf{G})-\{0, \mathbf{1}, \infty\}$. The map

$$
w: x \mapsto w_{2}(x) / w_{1}(x)
$$

is a multivalued map from $Q$ to the projective space $P\left(W^{*}\right)$ of lines in the dual space $W^{*}$ of $\mathrm{W}$, i.e. $w$ may be construed as a single-valued map

$$
\hat{w}: \hat{\mathrm{Q}} \rightarrow \mathrm{P}\left(\mathrm{W}^{*}\right) \text {. }
$$

The fundamental group $\pi_{1}(\mathrm{Q})$ acts on $\mathrm{P}\left(\mathrm{W}^{*}\right)$ (" monodromy action ") and the map $\hat{w}$ is $\pi_{1}(\mathrm{M})$-equivariant. Let $\Gamma$ denote the image of $\pi_{1}(\mathrm{Q})$ via the monodromy action. For Schwarz's original problem, the question reduces to "when is $\Gamma$ finite?" Schwarz also solved the problem: when can (4) be inverted to provide a univalued map from an open domain to $Q$ ? When this happens $\Gamma$ has a fundamental domain for its action on either (i) $\mathrm{P}\left(\mathrm{W}^{*}\right)$, (ii) $\mathrm{P}\left(\mathrm{W}^{*}\right)$ minus a point, or (iii) a disc in $\mathrm{P}\left(\mathrm{W}^{*}\right)$, and is consequently discrete in $\mathrm{PGL}_{2}(\mathbf{G})$.

It is case (iii) of this latter question which Picard generalized in [ $16 b]$ to a two variable analogue of the hypergeometric function. In the more general $d$ variable case, this function is best defined by its integral representation

$$
\mathrm{F}\left(x_{2}, \ldots, x_{d+1}\right)=\int_{1}^{\infty} u^{-\mu_{0}}(u-1)^{-\mu_{1}} \prod_{2}^{d+1}\left(u-x_{i}\right)^{-\mu_{i}} d u
$$

Let $\mu_{\infty}$ be the order of the pole of the integrand at $\infty$. When the sum is extended over all the $\mu$ 's, one has $\Sigma \mu_{i}=2$. In this introduction, we assume that none of the $\mu_{i}$ is an integer.

The function $\mathrm{G}$ obtained from $\mathrm{F}$ by holding fixed all variables but one was investigated by Pochhammer [ $\mathrm{I} 7]$. He formed the $(d+\mathrm{I})$-order linear differential equation satisfied by $G$ and characterized its solutions as the multivalued functions with exactly $d+$ I linearly independent branches and ramification of a prescribed type. At each finite ramification point, $d$ branches of $\mathrm{G}$ are holomorphic, and at $\infty$ the same holds true after multiplying $G$ by a suitable power of the variable. 
The function $\mathrm{F}$ defined by $\left(\mathbf{3}^{\prime \prime}\right)$ has a power series expansion

$$
\frac{\Gamma\left(2-\mu_{\infty}-\mu_{1}\right)}{\Gamma\left(1-\mu_{\infty}\right) \Gamma\left(1-\mu_{1}\right)} \mathrm{F}=\Sigma \frac{\prod_{2}^{d+1}\left(\mu_{i}, n_{i}\right) \cdot\left(1-\mu_{\infty}, \Sigma n_{i}\right)}{\left(2-\mu_{\infty}-\mu_{1}, \Sigma n_{i}\right)} \prod_{2}^{d+1} \frac{x_{i}^{n_{i}}}{n_{i} !} .
$$

For $d=2$, this series has been investigated by Appell [ $\mathrm{I} b]$ who showed that it satisfies a system of linear partial differential equations expressing each second derivative of $\mathrm{F}$ in terms of its first derivatives. In [ $\mathrm{I} 6 \mathrm{a}$ ] Picard characterized the solutions of this system as the multivalued functions of two variables $x, y$ with exactly three linearly independent branches and with ramification of a prescribed type along the seven lines $x$ or $y=0, \mathrm{I}, \infty, x=y$. The function $\mathrm{F}$ is the only solution holomorphic at $(0,0)$.

For $d$ arbitrary, the series ( $\left.\mathrm{I}^{\prime}\right)$ has been investigated by Lauricella [12], and Terada [22] obtained results parallel to those of Picard [ $[6 a]$.

The existence of differential or partial differential equations of Fuchsian type as above is to be expected if one considers $\left(3^{\prime \prime}\right)$ as a period integral. The equations arc satisfied not only by $\mathrm{F}$ but also by any integral of the same integrand taken from $g$ to $h$ where $g$ and $h$ are in $\left\{\infty, 0, \mathrm{I}, x_{2}, \ldots, x_{d+1}\right\}$. There are $d+\mathrm{I}$ linearly independent such integrals, and, following Schwarz, it is natural to take them as the projective coordinates of a point in projective the $d$-space $\mathbf{P}^{d}$. This yields a map

$$
\hat{w}: \hat{Q} \rightarrow \mathbf{P}^{d}
$$

where $\hat{Q}$ is the universal covering of the space $Q \subset\left(\mathbf{P}^{1}\right)^{d}$ defined as

$$
\mathrm{Q}=\left\{\left(x_{i}\right) \mid x_{i} \neq 0,1, \infty \text { and } x_{i} \neq x_{j} \text { for } i \neq j\right\} \text {. }
$$

The action of $\pi_{1}(Q)$ on $\mathbf{P}^{d}$ is called the monodromy action. The map $\left(4^{\prime}\right)$ is $\pi_{1}(\mathbf{Q})$ equivariant. Let $\Gamma$ denote the image of $\pi_{1}(Q)$ in $\operatorname{PGL}(d+1, \mathbf{C})$. In $[16 a]$ and $[16 b]$ Picard gives a criterion for the multivalued map $w: \mathbf{Q} \rightarrow \mathbf{P}^{d}(d=2)$ defined by $\left(4^{\prime}\right)$ to invert as a univalued map from a ball in $\mathbf{P}^{d}$ to a partial compactification of $\mathbf{Q}$. When this happens, $\Gamma$ is discrete in a $\operatorname{PU}(\mathrm{I}, d)$ subgroup of $\operatorname{PGL}(d+\mathrm{I}, \mathbf{C})$.

As pointed out in [ $14 a]$ the proof of discreteness of $\Gamma$ that Picard sketches in [ $16 a]$ leads to an obstacle and is inadequate. Our first objective in this paper is to give a correct proof for $d=2$ and also for general $d$. In order to carry this out in modern concepts, we nced only deal with rational $\mu_{i}$ and multivalued integrands on $\mathbf{P}^{1}$ which are single valued on a finite ramified covering of $\mathbf{P} 1$. However, in order to provide a framework for dealing with arbitrary parameters $\mu$, we have introduced local systems on $\mathbf{P}^{1}-\left\{\infty, 0, \mathrm{I}, x_{2}, \ldots, x_{d+1}\right\}$. This necessitates an $a b$ ovo development of holomorphic cohomology in order to validate the corresponding Hodge decomposition. In the end, this viewpoint does have the advantage of directness.

The two main theorems of this paper are Theorems (II.4) and (I2.II). The first says in effect: If $0<\mu_{i}<\mathrm{I}$ for all $i(0 \leq i \leq d+1$ or $i=\infty)$ and $\left(\mathrm{I}-\mu_{i}-\mu_{j}\right)^{-1}$ is an integer for all $i \neq j$ such that $\mu_{i}+\mu_{j}<\mathrm{r}$, then the mono- 
dromy group of the hypergeometric function $\left(3^{\prime \prime}\right)$ is a lattice in the projective unitary group $\mathrm{PU}(\mathrm{I}, d)$, i.e. is discrete and of finite covolume.

Theorem (I2.II) combined with (12.9) says in effect: For each non-arithmetic lattice arising in Theorem (I I .4), there is an algebraic family defined over $\mathbf{Q}$ of algebraic curves $X$ whose monodromy group is a subgroup of Aut $\mathrm{H}^{\mathbf{1}}\left(\mathrm{X}_{0}, \mathbf{Z}\right)$ which is not of finite covolume in its Zariski-closure.

The example 15 of $\S(14.3)$ corresponds for instance to the family of curves, depending on the parameters $x, y$, with equation

$$
v^{12}=u^{6}(u-\mathrm{I})^{5}(u-x)^{5}(u-y)^{4}
$$

(a cyclic covering of order I $_{2}$ of $\mathbf{P}^{\mathbf{1}}$ ).

The integrality condition that $\left(I-\mu_{i}-\mu_{j}\right)^{-1}$ be an integer ensures that the key map of this paper is etale in codimension one. A model situation in which integrality is used in such a way is the following. Let $\mathrm{D}$ be the unit disc, $\mathrm{D}^{*}:=\mathrm{D}-\{\mathrm{o}\}$, and $\alpha=r / s$ a rational number $>0$, written as a reduced fraction. Let $D^{* \sim}$ be the finite covering of $D^{*}$ on which the multivalued function $z \mapsto z^{\alpha}$ is defined. We complete it to the ramified covering $\mathrm{D}^{\sim}=\mathrm{D}^{* \sim} \cup\{0\}$ of $\mathrm{D}$. The multivalued function $z^{1 / s}$ on $\mathrm{D}^{*}$ " is " a uniformizing parameter for $\mathrm{D}^{\sim}$ at o. The map $z \mapsto z^{\alpha}$ from $\mathrm{D}^{\sim}$ to $\mathbf{C}$ is etale at o (i.e. etale in codimension I) if and only if $\alpha^{-1}$ is an integer. Indeed $z^{\alpha}=\left(z^{1 / s}\right)^{r}$. For a description of how this enters the proof, we refer to the comments after (3. I I).

The lists in Section I4 provide examples of non-arithmetic lattices in $\mathrm{PU}(\mathrm{I}, d)$ for $d=2,3$-both cocompact and non-cocompact for $d=2$. For $d>5$, Theorem (II.4) yields no lattice at all, by ( 14.2$)$.

The case $d=\mathrm{I}$ is treated in $(12.5 \cdot 5)$ and (12.6.3). The lattices arising from Theorem (I I.4) in this case are the triangle groups $[p, q, r]$ generated by rotations through double the angles with centers the vertex of a geodesic triangle in the Poincaré disc, with angles $\pi / p, \pi / q, \pi / r$ when $p, q, r$ are positive integers (or $\infty$ ) satisfying $\frac{\mathrm{I}}{p}+\frac{\mathrm{I}}{q}+\frac{\mathrm{I}}{r}<\mathrm{I}$. These lattices are described in Fricke-Klein [6] and are infinite in number.

However, the number of arithmetic lattices arising is finite. An explicit list can be deduced from results of Takeuchi on arithmetic triangle groups [2 I].

In the final Section I5, we make some comments about the list of Io2 solutions of R. Levavasseur in [ ${ }^{1} 3$ ] to Picard's integrality conditions for discreteness in the case $d=2$.

In the paper " Generalized Picard lattices arising from half-integral conditions", printed after this one, the second named author is able to relax the integrality condition on $\left(1-\mu_{i}-\mu_{j}\right)^{-1}$ and obtains lattices for $d \leq 9\left[\mathrm{I}_{4} b\right]$. 


\section{TABLE OF CONTENTS}

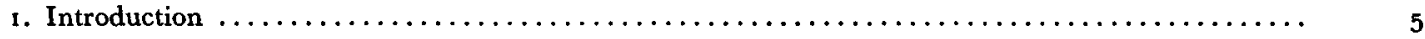

2. Cohomology of a rank one local system on a punctured complex line $\ldots \ldots \ldots \ldots \ldots \ldots \ldots$ 10

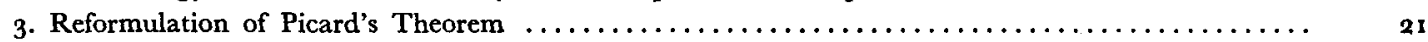

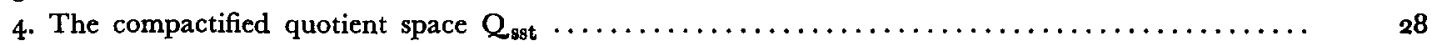

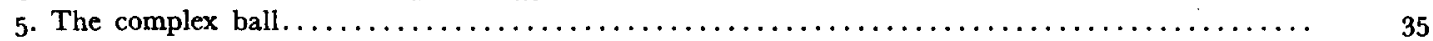

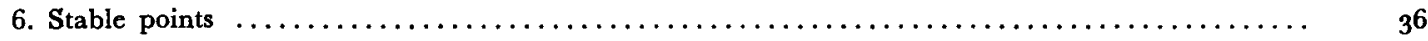

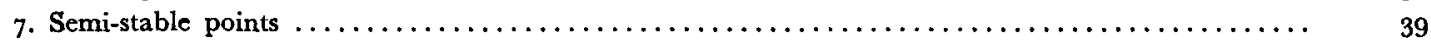

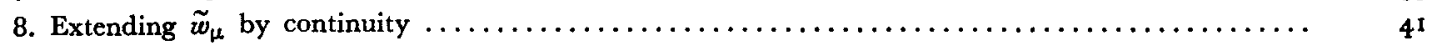

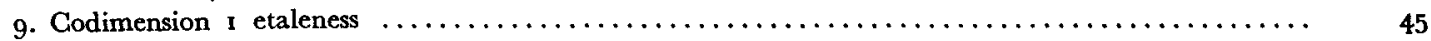

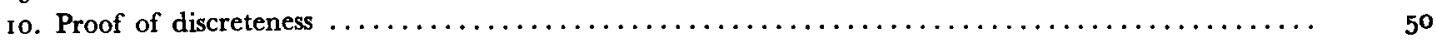

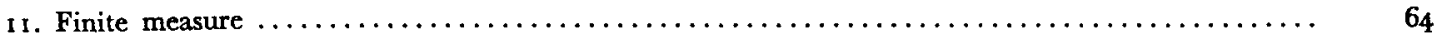

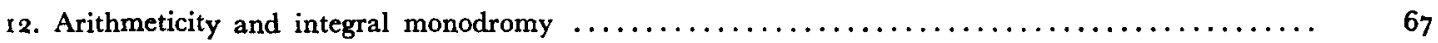

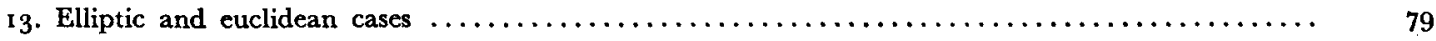

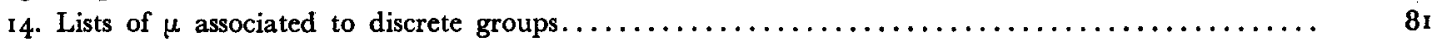

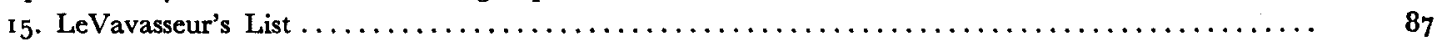

\section{Notations}

\begin{tabular}{|c|c|}
\hline Symbol & defined first in \\
\hline $\mathbf{P}$ & (2.1) \\
\hline $\mathbf{N}$ & $(2.1)$ \\
\hline $\mathbf{S}$ & (2.1) \\
\hline$\alpha$ & $(\mathbf{2} . \mathbf{I})$ \\
\hline $\mathbf{L}$ & $(2.1)(3.1)$ \\
\hline $\mathbf{L}^{V}$ & $(\mathbf{2} \cdot \mathbf{2})$ \\
\hline$\Omega^{*}(\mathbf{L})$ & $(2.7)$ \\
\hline $\mathrm{O}(\mathrm{L})$ & $(2.7)$ \\
\hline$\psi_{0}$ & $(2,18)$ \\
\hline$()$, & $(2.18)$ \\
\hline $\mathrm{H}^{1,0}$ & $(2.18)$ \\
\hline $\mathbf{M}$ & $(3 \cdot 1)$ \\
\hline $\mathbf{P}_{\mathbf{M}}$ & $(3 \cdot I)$ \\
\hline $\mathrm{L}_{m}$ & $(3 \cdot I)$ \\
\hline $\mathbf{B}(\alpha)_{M}$ & $(3 \cdot 2)$ \\
\hline$w_{\mu}$ & $(3 \cdot 4)$ \\
\hline G & $(3 \cdot 7)$ \\
\hline $\mathbf{Q}$ & $(3 \cdot 7)$ \\
\hline$\tilde{\mathbf{Q}}$ & $(3.8)$ \\
\hline $\mathbf{B}(\alpha)_{\mathbf{M}}^{+}$ & $(3 \cdot 10)$ \\
\hline $\mathrm{B}(\alpha)_{0}^{+}$ & $(3.10)$ \\
\hline$\theta$ & $(3.10)(3.15)$ \\
\hline$\theta^{\prime}$ & $(3 \cdot 15)$ \\
\hline INT & $(3.11)$ \\
\hline$\mu$ & $(4 \cdot 1)$ \\
\hline$Q_{\text {st }}, Q_{\text {sst }}$ & $(4 \cdot 1)$ \\
\hline$S(I), S(2)$ & $(7 \cdot 1)$ \\
\hline$\tilde{\mathbf{Q}}_{\mathrm{st}}, \tilde{\mathbf{Q}}_{\mathrm{sst}}$ & $(8.6)$ \\
\hline
\end{tabular}




\section{Cohomology of a rank one local system on a punctured projective line}

(2.1) Let us start with the following data:

$\mathbf{P}$ : a complex projective line;

$\mathrm{N}:$ an integer $>\mathrm{o}$;

$\mathrm{S}:$ a set of $\mathrm{N}$ points of $\mathrm{P}$;

$\alpha=\left(\alpha_{s}\right)_{s \in \mathrm{S}}$ : a family of complex numbers indexed by $\mathrm{S}$, satisfying the condition $\Pi \alpha_{s}=1$.

We will be mainly interested in the case when $N \geq 3$ and none of the $\alpha_{a}$ is $I$.

If a base point $o \in \mathbf{P}-\mathrm{S}$ is given, the functor "fibre at $o$ " is an equivalence of the category of complex local systems (= flat vector bundles) on $P$... $S$ with that of complex vector spaces provided with an action of $\pi_{1}(\mathbf{P}-\mathrm{S}, 0)$. In particular, rank one local systems correspond to homomorphisms $H_{1}(P-S)=\pi_{1}(P-S, o)^{a b} \rightarrow C^{*}$. The group $H_{1}$ is generated by small positive loops $\gamma_{8}$ around each $s \in S$, with the relation $\Sigma_{\gamma_{1}}=0$ as the only relation. Up to isomorphism, there is hence a unique one dimensional complex local system $L$ on $P$ - $-S$ such that the monodromy of $L$ around each $s \in S$ is multiplication by $\alpha_{s}$, or, as we will say, of monodromy $\alpha$. By definition, if $\ell_{0}$ is in the fibre of $\mathrm{L}$ at a point $x$ near $s \in \mathrm{S}$, and if we let $x$ turn counter clockwise once around $s$, and push $\ell_{0}$ horizontally along the path of $x$, then when coming back, $\ell_{0}$ becomes $\alpha_{1} l_{0}$. In other words, in term of a local coordinate $z$ centered at $s$, and of a multivalued section $e(z)$ of $\mathrm{L}$ defined in a neighborhood of $s$, one has

$$
\left.e(\exp (2 \pi i u) \cdot z)\right|_{u=1}=\left.\alpha_{s} \cdot e(\operatorname{cxp}(2 \pi i u) \cdot z)\right|_{u=0} .
$$

The complex local system $L$ has automorphisms: $\operatorname{Aut}(\mathrm{L})=\mathbf{C}^{*}$, with $v \in \mathbf{C}^{*}$ corresponding to multiplication by the scalar $v$. Because of them, even though the isomorphism class of $\mathrm{L}$ is uniquely detcrmined by the $x_{8}, \mathrm{~L}$ is not determined up to unique isomorphism.

Let us fix one L. In (2.2)-(2, I0), we will review some of the descriptions of the cohomology of $\mathrm{P}-\mathrm{S}$ with coefficients in $\mathrm{L}$.

(2.2) Combinatorial description. -- Let us fix a triangulation $\mathscr{E}$ of $\mathrm{P}-\mathrm{S}$. One can then identify $\mathrm{H}^{*}(\mathrm{P}-\mathrm{S}, \mathrm{L})$ with the cohomology of the complex of L-valued cochains of $\mathscr{E}$ : cochains $c$ for which the value of $c$ on an oriented simplex $\sigma$ is a horizontal section of $\mathrm{L}$ on $\sigma$ (thus $c(\sigma) \in \mathrm{H}^{0}(\sigma, \mathrm{L})$ ). To make sense of the formula $(d c)(\sigma)=c(b \sigma)$, one uses that a horizontal section of $\mathrm{L}$ on a face of $\sigma$ extends uniquely as a horizontal section on $\sigma$. This complex is the dual of the complex of chains of $\mathscr{E}$ with coefficients in the dual local system $L^{v}$; chains $C$ which are finite sums $\Sigma_{c_{\sigma}} \cdot \sigma$ with $c_{\sigma} \in \mathrm{H}^{0}\left(\sigma, \mathrm{L}^{\vee}\right)$ and $\sigma$ an oriented simplex of $\mathscr{E}$. The sign rule $\langle d c, \mathrm{C}\rangle=\langle c, b \mathrm{C}\rangle$ is however unusual for duality of complexes. We write $H_{*}\left(P-S, L^{\vee}\right)$ for the corresponding homology. 
The cohomology with compact support $\mathrm{H}_{c}^{*}(\mathbf{P}-\mathrm{S}, \mathrm{L})$ is the cohomology of the complex of compactly supported L-valued cochains of $\mathscr{E}$. The dual complex is the complex of locally finite chains with coefficient in $L^{\vee}$ : chains $\mathrm{C}$ which are possibly infinite "sums" $\Sigma c_{\sigma} . \sigma$. We write $\mathrm{H}^{\prime \prime}\left(\mathrm{P}-\mathrm{S}, \mathrm{L}^{v}\right)$ for the corresponding homology.

Let $\mathrm{X}$ be obtained from $\mathrm{P}$ by deleting a small open disc around each $s \in \mathrm{S}$. "Small" means "small enough". What matters here is that the closure of the discs be disjoint. A homotopy argument shows that $\left(\mathrm{X}^{0}\right.$ denoting $\left.\mathrm{X}-\partial \mathrm{X}\right)$

and that

$$
\mathrm{H}^{*}(\mathrm{P}-\mathrm{S}, \mathrm{L}) \stackrel{\sim}{\rightarrow} \mathrm{H}^{*}(\mathrm{X}, \mathrm{L}) \stackrel{\sim}{\rightarrow} \mathrm{H}^{*}\left(\mathrm{X}^{0}, \mathrm{~L}\right)
$$

To compute cohomology, one can hence use a triangulation of $\mathrm{X}$, instead of one of $\mathrm{P}-\mathrm{S}$. As $\mathrm{X}$ is compact, the triangulation is finite, and this combinatorial description makes clear that the Euler-Poincare characteristic

$$
\begin{aligned}
\chi(\mathrm{P}-\mathrm{S}, \mathrm{L}):=\Sigma(-\mathrm{I})^{i} \operatorname{dim} \mathrm{H}^{i}(\mathrm{P}-\mathrm{S}, \mathrm{L}) \\
\quad\left(\operatorname{resp.} \chi_{c}(\mathrm{P}-\mathrm{S}, \mathrm{L}):=\Sigma(-\mathrm{I})^{i} \operatorname{dim} \mathrm{H}_{c}^{i}(\mathrm{P}-\mathrm{S}, \mathrm{L})\right)
\end{aligned}
$$

is independent of $\mathrm{L}$. Each $\mathrm{H}^{i}$ (resp. $\mathrm{H}_{c}^{i}$ ) is indeed expressed as the $i$-th cohomology group of a finite dimensional chain complex $\mathrm{K}$, and $\operatorname{dim} \mathrm{K}^{i}$ is independent of $\mathrm{L}$. In the case of cohomology with compact support, the additivity of $\chi_{c}$ (deduced from the long exact sequence $\left.\ldots \rightarrow \mathrm{H}_{c}^{\mathrm{i}}(\mathrm{P}-\mathrm{S}, \mathbf{C}) \rightarrow \mathrm{H}^{\mathrm{i}}(\mathrm{P}, \mathbf{C}) \rightarrow \mathrm{H}^{\mathrm{i}}(\mathrm{S}, \mathbf{C}) \rightarrow \ldots\right)$ gives (2.2.x)

$$
\chi_{c}(\mathrm{P}-\mathrm{S}, \mathrm{L})=\chi_{c}(\mathrm{P}-\mathrm{S}, \mathbf{C})=\chi_{c}(\mathrm{P})-\chi_{c}(\mathrm{~S})=2-\mathrm{N} \text {. }
$$

For an algebraic variety $\mathrm{Y}$, one always has $\chi_{c}(\mathrm{Y})=\chi(\mathrm{Y})$. In the case at hand $(\mathrm{Y}=\mathrm{P}-\mathrm{S})$; this can be deduced from Poincaré duality. One gets

$$
\chi(\mathrm{P}-\mathrm{S}, \mathrm{L})=2-\mathrm{N} \text {. }
$$

(2.3) De Rham $\left(\mathrm{G}^{\infty}\right)$ description. - $\mathrm{H}^{*}(\mathrm{P}-\mathrm{S}, \mathrm{L})$ is the cohomology of the de Rham complex of L-valued $\mathrm{C}^{\infty}$ differential forms on $P-S$, and $H_{c}(P-S, L)$ that of the subcomplex of compactly supported forms. If the triangulation $\mathscr{E}$ of (2.2) is smooth, integration: $\omega \mapsto c_{\omega}(\sigma)=\int_{\sigma} \omega$ is defined. It is a map of complexes from the de Rham complex to the cochain complex (both with or without support condition), inducing an isomorphism on cohomology.

The Poincare duality pairing obtained by integration on $\mathrm{P}-\mathrm{S}: \alpha, \beta \mapsto \int_{\mathrm{P}-\mathrm{B}} \alpha \wedge \beta$ induces a perfect pairing

$$
\mathrm{H}^{i}(\mathrm{P}-\mathrm{S}, \mathrm{L}) \otimes \mathrm{H}_{c}^{2-i}\left(\mathrm{P}-\mathrm{S}, \mathrm{L}^{\vee}\right) \stackrel{\sim}{\rightarrow} \mathbf{C},
$$

the composite of cup product with value in $\mathrm{H}_{c}^{2}(\mathrm{P}-\mathrm{S}, \mathbf{C})$, and of the trace, or integration, map : $\mathrm{H}_{c}^{2}(P-S, C) \stackrel{\sim}{\rightarrow}$.

If at least one of the $\alpha_{z}$ is not $\mathrm{I}$, there can be no global horizontal section: $\mathrm{H}^{0}(\mathrm{P}-\mathrm{S}, \mathrm{L})=\mathrm{o}$, and a fortiori none with compact support: $\mathrm{H}_{c}^{0}(\mathrm{P}-\mathrm{S}, \mathrm{L})=\mathrm{o}$ 
(for this, $\mathrm{S} \neq \emptyset$ suffices). The same applies to the dual $\mathrm{L}^{\vee}$ of $\mathrm{L}$, with monodromy $\alpha^{-1}$, and Poincaré duality gives vanishing for $\mathrm{H}^{2}$ and $\mathrm{H}_{\mathfrak{c}}^{2}$. The Euler-Poincaré characteristic being known $((2.2 .1)$ and $(2.2 .2))$, we get

Proposition (2.3.1). - If $\alpha_{s} \neq \mathrm{I}$ for at least one $s \in \mathrm{S}$, then $\mathrm{H}^{\mathrm{i}}(\mathrm{P}-\mathrm{S}, \mathrm{L})$ and $\mathrm{H}_{c}^{i}(\mathrm{P}-\mathrm{S}, \mathrm{L})$ vanish for $i \neq \mathrm{I}$, and

$$
\operatorname{dim} \mathrm{H}^{1}(\mathbf{P}-\mathrm{S}, \mathrm{L})=\operatorname{dim} \mathrm{H}_{c}^{1}(\mathbf{P}-\mathrm{S}, \mathrm{L})==\mathrm{N}-2 .
$$

Currents (2.4). - Instead of using the $\mathrm{C}^{\infty}$ de Rham complex, consisting of forms written in local coordinates as $\sum_{1} f_{1} d x_{i_{1}} \wedge \ldots \wedge d x_{i_{k}} . e$, with $e$ an horizontal section of $\mathrm{L}$ and $f_{1}$ a $\mathbf{G}^{\infty}$-function, one can as well use the complex of currents, where $f_{1}$ is allowed to be a generalized function (distribution). This complex can be used to express Poincaré duality as a cap-product isomorphism between homology and cohomology: for $C_{\text {a }} L^{\vee}$-valued chain, there is a unique current $(G)$ such that $\int_{C} \omega=\int(C) \wedge \omega$, one has $d(\mathrm{G})=(-\mathrm{I})^{\operatorname{deg} c} b \mathrm{C}$, and the map $\mathrm{G} \mapsto(\mathrm{C})$ provides isomorphisms $\mathrm{H}_{i}(\mathbf{P}-\mathrm{S}, \mathrm{L}) \stackrel{\sim}{\rightarrow} \mathrm{H}_{c}^{2-i}(\mathrm{P}-\mathrm{S}, \mathrm{L})$ and $\mathrm{H}_{i}^{\ell}(\mathrm{P}-\mathrm{S}, \mathrm{L}) \stackrel{\sim}{\rightarrow} \mathrm{H}^{2-i}(\mathrm{P}-\mathrm{S}, \mathrm{L})$.

It is often more convenient to use currents than the chains of a fixed triangulation. If $\beta$ is a rectifiable proper map from an open, semi-open or closed interval $I$ to $P-S$, and for $e \in \mathrm{H}\left(\mathrm{I}, \beta^{*} \mathrm{~L}^{\vee}\right)$, we let $(e . \beta)$ be the $\mathrm{L}^{\mathrm{V}}$-valued current for which

$$
\int(e . \beta) \wedge \omega=\int_{I}\left\langle e, \beta^{*} \omega\right\rangle \text {. }
$$

If $\beta:[0, I] \rightarrow P$ maps 0 and $I$ to $S$, and $] 0, I\left[\right.$ to $P-S$, then for $e \in \mathrm{H}^{0}(] 0, I\left[, \beta^{*} L^{\vee}\right)$, e. $\beta$ is a cycle and as such defines an homology class in $H_{1}^{\prime \prime}\left(P-S, L^{\vee}\right)$. We will use such cycles to construct convenient bases of $H_{1}^{\ell f}\left(P-S, L^{\vee}\right)$.

(2.5) Assume the following is given:

a) a partition of $S$ into two subsets $S_{1}$ and $S_{2}$;

b) trees $T_{1}$ and $T_{2}$ (a tree is a contractible $\mathrm{CW}$ complex of dimension $\leq_{1}$ ), and an embedding $\beta: T_{1} \amalg T_{2} \hookrightarrow P$, mapping the set of vertices of $T_{1}$ (resp. $T_{2}$ ) onto $S_{1}$ (resp. $\mathrm{S}_{2}$ );

c) for each (open) edge $a$ of $T_{1}$ or $T_{2}$, an orientation of $a$, and $\ell(a) \in \mathrm{H}^{0}\left(a, \beta^{*} \mathrm{~L}^{\vee}\right)$.

For each edge $a, \ell(a) \cdot \beta \mid a$ is then a locally finite cycle on $\mathrm{P}-\mathrm{S}$, with cocffcient in $\mathrm{L}$.

Proposition (2.5.1). - If $\prod_{s \in \mathrm{S}_{\mathbf{1}}} \alpha_{s} \neq \mathrm{I}$, the elements $\ell(a) \cdot \beta \mid a$, for $a$ an edge of $\mathrm{T}_{\mathbf{1}}$ or $\mathrm{T}_{2}$, form a basis of $\mathrm{H}_{1}^{\prime \prime}\left(\mathrm{P}-\mathrm{S}, \mathrm{L}^{\vee}\right)$.

Proof. - Let $\mathrm{T}_{i}^{\prime}$ be the disjoint union of the (open) cdges of $\mathrm{T}_{i}$. We have $(\mathrm{P}-\mathrm{S})-\beta\left(\mathrm{T}_{1}^{\prime} \amalg \mathrm{T}_{2}^{\prime}\right)=\mathrm{P}-\beta\left(\mathrm{T}_{1} \amalg \mathrm{T}_{2}\right)$, hence a long exact sequence

$$
\begin{aligned}
\ldots \rightarrow \mathrm{H}_{i}^{\prime \prime}\left(\mathrm{T}_{1}^{\prime} \amalg \mathrm{T}_{2}^{\prime}, \beta^{*} \mathrm{~L}^{\vee}\right) \stackrel{\beta}{\rightarrow} \mathrm{H}_{i}^{\ell \prime}(\mathrm{P}-\mathrm{S}, \mathrm{L}) & \\
& \rightarrow \mathrm{H}_{i}^{\ell \prime}\left(\mathrm{P}-\beta\left(\mathrm{T}_{1} \amalg \mathrm{T}_{2}\right), \mathrm{L}^{\vee}\right) \rightarrow \ldots
\end{aligned}
$$


The group $\mathrm{H}_{1}^{\prime \prime}\left(\mathrm{T}_{1}^{\prime} \amalg \mathrm{T}_{2}^{\prime}, \beta^{*} \mathrm{~L}^{\vee}\right)$ has as basis the $\ell(a) . a$. It remains to be proved that $\mathrm{H}_{*}^{\ell f}\left(\mathrm{P}-\beta\left(\mathrm{T}_{1} \amalg \mathrm{T}_{2}\right), \mathrm{L}\right)=\mathrm{o}$.

The space $P-\beta\left(T_{1}\right)-\beta\left(T_{2}\right)$ is the complement of two disjoint trees in $S^{2}$. It is essentially an annulus. The monodromy of " turning around $T_{1}$ " is the product of the $\alpha_{s}\left(s \in \mathrm{S}_{1}\right)$. In $\mathrm{P}-\mathrm{S}$, a loop around $\mathrm{T}_{1}$ can indeed be homotoped to a product of loops around each $s \in \mathrm{S}_{1}$ :
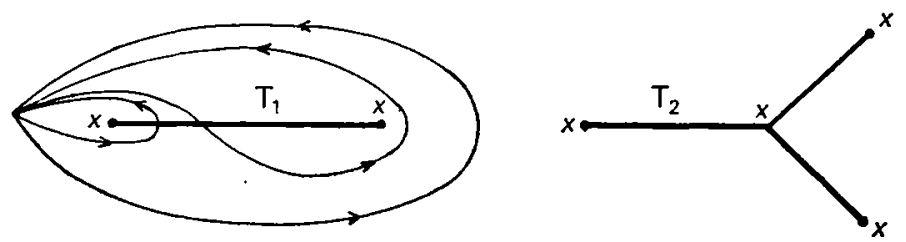

The group $H_{i}^{\prime \prime}\left(P-\beta\left(T_{1} \cup T_{2}\right), L^{\vee}\right)$ is dual to $H_{c}^{i}\left(P-\beta\left(T_{1} \cup T_{2}\right), L\right)$, which is the Poincaré dual of $H^{2-i}\left(P-\beta\left(T_{1} \cup T_{2}\right), L^{\vee}\right)$. One can homotope $P-\beta\left(T_{1} \cup T_{2}\right)$ to $S^{1}$, and the group becomes $\mathrm{H}^{2-i}\left(\mathrm{~S}^{1}, \mathrm{~L}^{\vee}\right)$, for $\mathrm{L}^{\vee}$ a non trivial local system on $\mathrm{S}^{1}$. It remains to use the

Lemma (2.5.2). - If $\mathrm{L}$ is a non trivial rank one local system on $\mathrm{S}^{1}$, then $\mathrm{H}^{*}\left(\mathrm{~S}^{1}, \mathrm{~L}\right)=0$.

The $\mathrm{H}^{0}$ is o by non triviality, and $\chi\left(\mathrm{S}^{1}, \mathbf{L}\right)=\chi\left(\mathrm{S}^{1}\right)=0$.

Remark. - As $\mathrm{T}_{1} \amalg \mathrm{T}_{2}$ has $\mathrm{N}-2$ edges, (2.5.2) reproves that

$$
\operatorname{dim} \mathrm{H}_{c}^{1}(\mathrm{P}-\mathrm{S}, \mathrm{L})=\mathrm{N}-2
$$

when the $\alpha_{s}$ are not all I.

(2.6) Sheaf cohomology. - We will always identify a local system L with its sheaf of locally constant sections. Let $j$ denote the inclusion of $\mathbf{P}-\mathrm{S}$ in $\mathrm{P}$. Then, $\mathrm{H}_{c}^{*}(\mathrm{P}-\mathrm{S}, \mathrm{L})$ is the cohomology of $\mathrm{P}$ with coefficients in $j_{1} \mathrm{~L}$, the extension of $\mathrm{L}$ by 0 . It is the hypercohomology on $\mathrm{P}$ of any complex of sheaves $\mathrm{K}$ with $\mathscr{H}^{0}(\mathrm{~K})=j_{1} \mathrm{~L}$ and $\mathscr{H}^{i}(\mathrm{~K})=\mathrm{o}$ for $i \neq 0$. On the other hand, if $\mathrm{L}^{*}$ is any resolution of $\mathrm{L}$ whose components are acyclic for $j_{*}\left(\mathrm{R}^{q} j_{*} \mathrm{~L}^{k}=\mathrm{o}\right.$ for $\left.q>0\right)$, then $\mathrm{H}^{*}(\mathrm{P}-\mathrm{S}, \mathrm{L})$ is the hypercohomology on $\mathrm{P}$ of $j_{*} \mathrm{~L}^{*}$. To prove it, one reduces to the case where $\mathrm{L}^{*}$ (and hence $j_{*} \mathrm{~L}^{*}$ ) is soft, one has $\Gamma\left(\mathrm{P}-\mathrm{S}, \mathrm{L}^{*}\right)=\Gamma\left(\mathrm{P}, j * \mathrm{~L}^{*}\right)$, and

$$
\mathrm{H}^{*}(\mathrm{P}-\mathrm{S}, \mathrm{L}):=\mathrm{H}^{*} \Gamma\left(\mathrm{P}-\mathrm{S}, \mathrm{L}^{*}\right)=\mathrm{H}^{*} \Gamma\left(\mathrm{P}, j_{*} \mathrm{~L}^{*}\right)=: \mathbf{H}^{*}\left(\mathrm{P}, j_{*} \mathrm{~L}^{*}\right) \text {. }
$$

Proposition (2.6.I). - If all $\alpha_{\mathrm{s}}$ are different from $\mathrm{I}$, then

$$
\mathrm{H}_{c}^{*}(\mathbf{P}-\mathrm{S}, \mathrm{L}) \stackrel{\sim}{\rightarrow} \mathrm{H}^{*}(\mathbf{P}-\mathrm{S}, \mathrm{L}) \text {. }
$$

Proof. - Let $\mathrm{L}^{*}$ be a soft resolution of $\mathrm{L}$, for instance the $\mathrm{L}$-valued $\mathrm{G}^{\infty}$ de $\mathrm{Rham}$ complex of sheaves. It suffices to check that $j_{*} \mathrm{~L}^{*}$ is a resolution of $j_{l} \mathrm{~L}$, and this results from the local fact that for $\mathrm{D}$ any small disc around $s \in \mathrm{S}$, one has

$$
\mathrm{H}^{*} \Gamma\left(\mathrm{D}-s, \mathrm{~L}^{*}\right)=\mathrm{H}^{*}(\mathrm{D}-s, \mathrm{~L})=\mathrm{o} \text {. }
$$

To check this vanishing, one can replace $\mathrm{D}-s$ by a homotopic $\mathrm{S}^{\mathbf{1}}$ and apply (2.5.2). 
From the point of view of homology, this implies that a locally finite I-cycle with coefficient in $\mathrm{L}^{\vee}$ is homologous to a compactly supported one. Here is a drawing of how it can be done

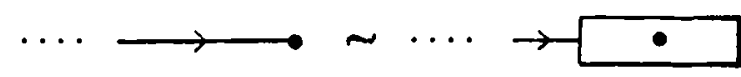

This is reminiscent of the arguments used in Whittaker and Watson (12.22) (Hankel's expression of $\Gamma(z)$ as a contour integral) or (12.43) [24].

(2.7) The de Rham (holomorphic) description. - The holomorphic L-valued de Rham complex $\Omega^{*}(\mathrm{~L}): \mathcal{O}(\mathrm{L}) \rightarrow \Omega^{1}(\mathrm{~L})$ is a resolution of $\mathrm{L}$ on $\mathrm{P}-\mathrm{S}$. One can hence interpret $\mathrm{H}^{*}(\mathrm{P}-\mathrm{S}, \mathrm{L})$ as the hypercohomology on $\mathrm{P}-\mathrm{S}$ of $\Omega^{*}(\mathrm{~L})$. As $\mathrm{S} \neq \varnothing$, $\mathbf{P}-\mathrm{S}$ is Stein, hence $\mathrm{H}^{q}\left(\mathbf{P}-\mathrm{S}, \Omega^{p}(\mathrm{~L})\right)=0$ for $q>0$, and this gives

$$
H^{*}(P-S, L)=H^{*} \Gamma\left(P-S, \Omega^{*}(L)\right) \text {. }
$$

Similarly, $\mathrm{R}^{q} j \Omega^{p}(\mathrm{~L})=\mathrm{o}$ for $q>0$ hence

$$
\mathrm{H}^{*}(\mathrm{P}-\mathrm{S}, \mathrm{L})=\mathbf{H}^{*}\left(\mathrm{P}, j, \Omega^{*}(\mathrm{~L})\right)
$$

(hypercohomology of $\mathrm{P}$, with coefficients in the complex $j, \Omega^{*}(L)$; see (2.6)).

After the preliminaries (2.8), (2.9), we will show how to replace $j_{.} \Omega^{*}(\mathrm{~L})$ by smaller complexes of sheaves.

(2.8) We will often describe a section of $\mathcal{O}(\mathrm{L})$ on a connected open set $\mathrm{U}$ as the product of a multivalued function with a multivalued section of $L$. This has the following meaning: $U$ should be provided with a base point $o$, and a multivalued section of a sheaf $\mathscr{F}(\mathcal{O}$, or $\mathrm{L}, \ldots)$ is a section of the pull back of $\mathscr{F}$ on the universal coverings $(\hat{\mathrm{U}}, o)$ of $(\mathrm{U}, o)$. Products are taken on $\hat{\mathrm{U}}$. A multivalued section, which is the pull-back of a section on $\mathrm{U}$, is then identified with that section. The role of the basc point can be played by a contractible subset of $F$ of $U$. A section of $L$ at $o$ extends uniquely to a multivalued section and will be denoted by the same symbol. A multivalued section of $\mathcal{O}$ is determined by its germ at 0 , and will be denoted by the same symbol. As multivalued sections of $\mathcal{O}$, we will mainly use products $\Pi g_{i}^{\mu_{i}}$. When $g$ is not real and negative, the principal determination of $g^{\mu}$ is $\exp (2 \pi i \mu \log g)$, with $|\operatorname{Im} \log g|<\pi i$. As a rule, $g_{i}^{\mu}$ will denote the multivalued function whose germ at the base contractible set $\mathrm{F}$ is the principal determination when $g_{i}$ is not real and negative on F. When the determination used is irrelevant, we will not take the trouble of specifying which one we mean.

(2.9) Fix $s \in \mathrm{S}$ and let $z$ be a local coordinate, which we take to be an isomorphism, carrying $s$ to o, of a small neighborhood D of $s$ with a disc in $\mathbf{C}$ centered at 0 . If $\mu \in \mathbf{C}$ is such that $\alpha_{s}=\exp (2 \pi i \mu)$, the monodromy of $z^{-\mu}$ around $s$ is the inverse of that of a horizontal section of $\mathrm{L}$. Any section $u$ of $\mathcal{O}(\mathrm{L})$ (resp. $\Omega^{1}(\mathrm{~L})$ ) on $\mathrm{D}^{*}$ can hence be written $u=z^{-\mu} . e . f$ (resp. $u=z^{-\mu} . e . f . d z$ ) with $e$ a non zero multi- 
valued section of $\mathrm{L}$ and $f$ a holomorphic function on $\mathrm{D}^{*}$. We define $u$ to be meromorphic at $s$ if $f$ is, and we define its valuation by

$$
v_{s}(u)=v_{s}(f)-\mu \text {. }
$$

These definitions are independent of the choices of local coordinate and of $\mu$.

(2.10) The de Rham (meromorphic at S) description. - Let us write $j_{*}^{m} \Omega^{*}(\mathrm{~L})$ for the subcomplex of $\Omega^{*}(\mathrm{~L})$ of meromorphic forms. A local computation around each $s \in \mathrm{S}$ shows that the inclusion of $j_{*}^{m} \Omega^{*}(\mathrm{~L})$ in $j_{*} \Omega^{*}(\mathrm{~L})$ induces an isomorphism on the cohomology sheaves. This implies that

$$
\text { (2. 10. I) } \quad \mathbf{H}^{*}\left(\mathrm{P}, j_{*}^{m} \Omega^{*}(\mathrm{~L})\right) \stackrel{\sim}{\rightarrow} \mathbf{H}^{*}\left(\mathrm{P}, j_{*} \Omega^{*}(\mathrm{~L})\right)=\mathrm{H}^{*}(\mathrm{P}-\mathrm{S}, \mathrm{L}) \text {. }
$$

It follows from (2.1I) below that $j_{*}^{m} \Omega^{p}(\mathrm{~L})$ is an inductive limit of line bundles whose degrees tends to $\infty$. From this one concludes first that $\mathrm{H}^{q}\left(\mathbf{P}, j_{*}^{m} \Omega^{p}(\mathrm{~L})\right)=0$ for $q>0$, and then that $\mathbf{H}^{*}\left(\mathrm{P}, j_{*}^{m} \Omega^{*}(\mathrm{~L})\right)$ is simply $\mathrm{H}^{*} \Gamma\left(\mathrm{P}, j_{*}^{m} \Omega^{*}(\mathbf{L})\right)$, the cohomology of the complex of L-valued forms holomorphic on $\mathrm{P}-\mathrm{S}$ and meromorphic at each $s \in \mathrm{S}:$

$$
\mathrm{H}^{*}(\mathrm{P}-\mathrm{S}, \mathrm{L})=\mathrm{H}^{*} \Gamma\left(\mathrm{P}, j_{*}^{m} \Omega^{*}(\mathrm{~L})\right)
$$

(2.1x) Let $\left(\mu_{s}\right)_{s \in \mathrm{s}}$ be a family of complex numbers, such that $\exp \left(2 \pi i \mu_{s}\right)=\alpha_{\mathrm{s}}$. We define the line bundle $\mathcal{O}\left(\Sigma \mu_{s} s\right)(\mathrm{L})$ as the subsheaf of $j_{*}^{m} \mathcal{O}(\mathrm{L})$ whose local holomorphic sections are the local sections $u$ of $j_{*}^{m} \mathcal{O}(\mathrm{L})$ such that for $s \in \mathrm{S}$, the integer $v_{s}(u)+\mu_{s}$ is $\geq 0$ (in short: $v_{s}(u) \geq-\mu_{s}$ ). With the local coordinate notation (2.9), $z^{-\mu_{s} . e}$ determines an invertible section of $\mathcal{O}\left(\Sigma \mu_{s} s\right)(\mathrm{L})$ near $s \in \mathrm{S}$. The degree of a meromorphic section $u$ of a line bundle $\mathscr{L}$ at a point $x$ is the order of the zero (or minus the order of the pole) of $u$ at $x$; for $z$ a local coordinate centered at $x$, it is the supremum of the integers $n$ such that $z^{-n} u$ is a holomorphic section of $\mathscr{L}$ at $x$. The degree of $\mathscr{L}$ is the sum of the degrees at all points of any non zero meromorphic section of $\mathscr{L}$. If $u$ is a meromorphic section of $\mathcal{O}\left(\Sigma \mu_{s} s\right)(\mathrm{L})$ and $x \in \mathrm{S}$, one has

$$
\operatorname{deg}_{x}(u)=v_{x}(u)+\mu_{x} \text {. }
$$

If onc defines $\mu_{x}=0$ for $x \in \mathrm{P}-\mathrm{S}$, the same holds at any $x \in \mathrm{P}$.

Proposition (2.11.1). - The line bundle $\mathcal{O}\left(\Sigma_{\mu_{s}} s\right)(\mathrm{L})$ is of degree $\Sigma_{\mu_{s}}$.

Proof. - We may assume $\mathbf{P}=\mathbf{P}^{1}$ and $\infty \notin \mathrm{S}$. Let $e$ be a multivalued horizontal section of $\mathrm{L}$. The product

$$
u=\prod_{s \in \mathrm{S}}(z-s)^{-\mu_{s}, e}
$$

is an invertible section of $\mathscr{O}\left(\Sigma \mu_{s} . s\right)(\mathrm{L})$ on the affine line. At $\infty, u$ is meromorphic, of valuation $\Sigma \mu_{s}$. The degree of $\mathcal{O}\left(\Sigma \mu_{s} s\right)(\mathrm{L})$, equal to the sum at all $x \in \mathrm{P}$ of the degree of any non zero meromorphic section, is hence $\Sigma \mu_{s}$. 
We define $\Omega^{1}\left(\Sigma \mu_{8} . s\right)(\mathrm{L}):=\mathcal{O}\left(\Sigma \mu_{8} . s\right)(\mathrm{L}) \otimes \Omega^{1}$. It results from (2.I I) that (2.II.I) $\operatorname{deg} \Omega^{1}\left(\Sigma \mu_{1}, s\right)(\mathrm{L})=-2+\Sigma \mu_{i}$.

In particular, if $\Sigma \mu_{i}=2$, the line bundle $\Omega^{1}\left(\Sigma \mu_{3} . s\right)(L)$ is of degree o, hence isomorphic to $\mathcal{O}$, the trivial line bundle.

Corollary (2.12). - If $\Sigma \mu_{s}=2$, there is up to a constant factor one and only one nori zero form $\omega \in \Gamma\left(P, j_{*}^{m} \Omega^{1}(\mathrm{~L})\right)$ whose valuation at $s \in \mathrm{S}$ is $\geq-\mu_{\mathrm{s}}$. One has $v_{s}(\omega)=-\mu_{\mathrm{s}}$ and $\omega$ is invertible on $\mathrm{P}-\mathrm{S}$.

For $\mathbf{P}=\mathbf{P}^{1}, \infty \notin \mathrm{S}$, and $e$ a multivalued horizontal section of $\mathrm{L}$, one has (up

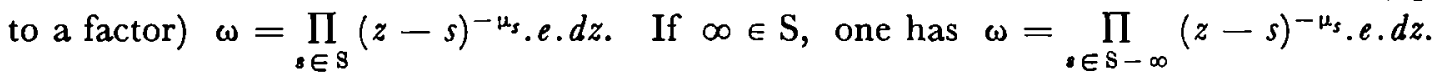

Proposition (2.13). - Assume that $\Sigma_{\mu_{s}}=2, \mathrm{~N} \geq 3$, and that none of the $\alpha_{8}$ is $\mathrm{I}$ (i.e. that none of the $\mu_{s}$ is an integer). Then the cohomology class of the form $\omega$ of (2.12) is not zero.

Proof. - One has $\Sigma v_{s}(\omega)=\Sigma-\mu_{s}=-2 \geq I-N$. The proposition hence results from the

Proposition (2.14). - Assume that none of the $\alpha_{\mathrm{s}}$ is $\mathrm{I}$. If a non zero form $\omega$ is such that $\Sigma v_{s}(\omega) \geq \mathrm{I}-\mathrm{N}$, its cohomology class is not zero.

Proof. - By (2.10.2), it suffices to verify that the equation $\omega=d u$ has no solution $u \in \Gamma\left(\mathrm{P}, j_{*}^{m} \mathcal{O}(\mathrm{L})\right)$.

For any local section $u$ of $j_{\bullet}^{m} \mathcal{O}(\mathrm{L})$ near $s$, one has

$$
v_{s}(d u) \geq v_{s}(u)-\mathrm{I},
$$

with equality if $v_{s}(u)$ is not $o$, or if $v_{s}(d u)$ is not a positive (meaning $\geq 0$ ) integer. The integer case being excluded, a solution $u$ would be a section of $\mathcal{O}\left(-\Sigma\left(v_{s}(\omega)+\mathrm{I}\right) s\right)(\mathbf{L})$, a line bundle of degree $-\Sigma\left(v_{s}(\omega)+1\right) \leq-1$, a contradiction.

(2.15) Here is how (2.13), (2.14) has to be modified for integral $\mu$ 's. As the result will not be needed, except for some historical comments in $\S$ I $_{5}$, we will be sketchy.

(2.15.1) When some $\alpha_{s}$ are $\mathrm{I},(2.6 . \mathrm{I})$ is not true, one has to distinguish between $\mathrm{H}_{c}^{1}$ and $H^{1}$, and even to introduce some intermediate groups: for $S^{\prime} C S$, the family $\Phi\left(S^{\prime}\right)$ of subsets of $\mathbf{P}-\mathrm{S}$ closed in $\mathbf{P}-\mathrm{S}^{\prime}$ is a family of supports, and $\mathrm{H}_{\Phi\left(\mathrm{S}^{\prime}\right)}^{*}(\mathbf{P}-\mathrm{S}, \mathrm{L})$ is defined. For $j^{\prime}$ the inclusion of $\mathbf{P}-\mathrm{S}$ into $\mathbf{P}-\mathrm{S}^{\prime}$, it is $\mathrm{H}^{*}\left(\mathbf{P}-\mathrm{S}^{\prime}, j_{1}^{\prime} \mathrm{L}\right)$. For $\mathrm{T} \subset \mathrm{S}$, define $\mathrm{T}(\mathrm{I}):=\left\{s \in \mathrm{T} \mid \alpha_{\mathrm{s}}=\mathrm{I}\right\}$. The proof of (2.6.I) shows that

$$
\mathrm{H}_{\Phi\left(\mathrm{S}^{\prime}(1)\right)}^{*}(\mathrm{P}-\mathrm{S}, \mathrm{L}) \stackrel{\sim}{\rightarrow} \mathrm{H}_{\Phi_{\left(\mathbf{S}^{\prime}\right)}}^{*}(\mathrm{P}-\mathrm{S}, \mathrm{L}) \text {. }
$$

These groups are also the hypercohomology groups, on $\mathrm{P}$, of the subcomplex of $j_{*}^{m} \Omega^{*}(\mathrm{~L})$ consisting of the $u$ in $j_{*}^{m}(\mathrm{~L})$ and of the $\omega$ in $j_{*}^{m} \Omega^{1}(\mathrm{~L})$ such that $v_{s}(\omega) \geq 0$ 
and $v_{s}(u)>0$ for $s \in\left(S-S^{\prime}\right)(1)$. If the $\mu_{s}$ are such that $\alpha_{s}=\exp \left(2 \pi i \mu_{s}\right)$, and that $\mathrm{S}^{\prime}(\mathrm{I})$ is the set of $s$ for which $\mu_{\mathrm{s}}$ is integral $>0$, the groups $\mathrm{H}_{\Phi\left(\mathrm{S}^{\prime}\right)}(\mathrm{P}-\mathrm{S}, \mathrm{L})$ are also the hypercohomology groups of the sub-complex $\mathcal{O}\left(\Sigma\left(\mu_{\mathrm{s}}-\mathrm{I}\right) s\right)(\mathrm{L}) \rightarrow \Omega^{1}\left(\Sigma_{\mathrm{s}} s\right)(\mathrm{L})$ of $j_{*}^{m} \Omega^{*}(\mathrm{~L})$.

(2.15.2) If $\omega \in \Gamma\left(\mathrm{P}, j^{m} \Omega^{1}(\mathrm{~L})\right)$, and if all $s$ with $\alpha_{s}=1$ and $v_{s}(\omega)<0$ are in $S^{\prime}$, then $\omega$ defines a cohomology class in $H_{\Phi\left(S^{\prime}\right)}^{1}(P-S, L)$. If in addition $S^{\prime}$ contains none of the $s$ for which simultaneously $a_{s}=\mathrm{I}$ and $v_{s}(\omega) \geq 0$, and if further $\Sigma v_{s}(\omega) \geq \mathrm{I}-\mathrm{N}$ (which amounts to $\omega$ having at most $\mathrm{N}-3$ zeros, counted with multiplicities, on $\mathrm{P}-\mathrm{S}$ ), the proof of (2.14) still shows that the class of $\omega$ in $H_{\Phi\left(S^{\prime}\right)}^{1}(P-S, L)$ is not zero. This applies to the form $\omega$ of (2.12), with $S^{\prime}=S$, provided $N \geq 3$ and $\mu_{s}>0$ whenever $\mu_{\varepsilon} \in \mathbf{Z}$.

Remark (2.16). - Let us assume that none of the $\alpha_{\mathrm{s}}$ is I. If $\omega$ is an holomorphic section of $\Omega^{1}(L)$ on $P-S$, by (2.6.I), the class $[\omega]$ of $\omega$ in $H^{1}(P-S, L)$ is the image of a unique class, again denoted $[\omega]$, in $\mathrm{H}_{c}^{1}(\mathbf{P}-\mathrm{S}, \mathrm{L})$. For any locally finite cycle or current $\mathbf{C}$ with coefficient in $\mathrm{L}^{\vee},\langle[\mathrm{G}],[\omega]\rangle$ is defined: $\mathrm{H}_{1}^{\ell f}\left(\mathbf{P}-\mathrm{S}, \mathrm{L}^{\vee}\right)$ is indeed paired with $\mathrm{H}_{c}^{1}(\mathbf{P}-\mathrm{S}, \mathrm{L})$. We want to prove that

$$
\langle[\mathrm{C}],[\omega]\rangle=\int_{\mathrm{C}} \omega \text {. }
$$

This is not completely obvious, especially since the integral may be divergent. The problem is rather to understand which value the cohomological formalism attaches to the integral.

Ist answer: One replaces $\mathbf{C}$ by a finite homologous cycle $\mathbf{C}^{\prime}$, as pictured in (2.6), and $\langle[\mathrm{C}],[\omega]\rangle=\int_{\mathrm{C}^{\prime}} \omega$.

2nd answer: If $\mathrm{D}_{s}$ is a small open disk around $s \in \mathrm{S}$, one has $\mathrm{H}^{*}(\mathrm{D}-s, \mathrm{~L})=0$ (cf. (2.6)). On $\mathrm{D}_{s}^{*}:=\mathrm{D}-s$, $\omega$ is hence of the form $d u_{s}$, for a unique section $u_{s}$ of $\mathcal{O}(\mathrm{L})$ on $\mathrm{D}_{\varepsilon}^{*}$. Let $\varphi_{s}$ be a compactly supported $\mathrm{G}^{\infty}$ function on $\mathrm{D}_{s}$, equal to $\mathrm{I}$ in a neighborhood of $s$. The $\mathrm{C}^{\infty}$ I-form $\omega-\Sigma d\left(\varphi_{s} u_{s}\right)$ is cohomologous to $\omega$ and is compactly supported. One has hence

$$
\langle[\mathrm{C}],[\omega]\rangle=\int_{\mathrm{C}} \omega-\Sigma d\left(\varphi_{s} u_{s}\right) .
$$

It is sometimes more convenient to take for $\varphi$ the (discontinuous) characteristic function of $\mathrm{D}_{s}$. The current $\omega-\Sigma d\left(\varphi_{s} u_{s}\right)$ is again compactly supported and cohomologous to $\omega$.

These formulae can also be used to define the integral of $\omega$ on a locally finite chain or current, which is a cycle only near each $s \in \mathrm{S}$. Given for instance a path $\beta:[0,1] \rightarrow P$, 
with $\beta\left(\left[0, \mathrm{I}[)\right.\right.$ in $\mathrm{P}-\mathrm{S}$ and $\beta(\mathrm{I})=s \in \mathrm{S}$, and given $e \in \mathrm{H}^{0}\left(\left[0, \mathrm{I}\left[, \beta^{*} \mathrm{~L}^{\vee}\right)\right.\right.$, the formula for the "finite part" $\int_{e . \beta} \omega-d\left(\varphi, u_{s}\right)$ can be rewritten

$$
\mathrm{P} f \int_{e . \beta} \omega=\int_{0}^{1-c}\left\langle e, \beta^{*} \omega\right\rangle-\left\langle e(a t \mathrm{I}-\varepsilon), \beta^{*} u_{\theta}(\text { at } \mathrm{I}-\varepsilon)\right\rangle .
$$

For a meromorphic $\omega$, this agrees with Hadamard's " finite part" of a divergent integral.

Example (2.17). - We take $\mathbf{P}=\mathbf{P}^{1}, \mathrm{~S}=\{0,1, \infty\}$ and we assume that none of the $\mu_{s}$ is an integer, and that $\Sigma \mu_{s}=2$. For $\lambda_{s}=I-\mu_{s}$, this means $\Sigma \lambda_{s}=I$. We normalize $\mathrm{L}$ by fixing a section $e$ of it on ]o, I [, and we let $e^{\vee}$ be the dual base of $\mathrm{L}^{\vee}$ on $] \mathrm{o}, \mathrm{I}\left[\right.$. Take $\omega=z^{\lambda_{0}-1}(1-z)^{\lambda_{1}-1} . e d z$; the " principal" determination of $z^{\lambda_{0}-1}$ and of $(\mathrm{I}-z)^{\lambda_{1}-1}$ on $] \mathrm{o}, \mathrm{I}[$ is used (cf. (2.8)). By (2.5.I), the homology $\mathrm{H}_{1}^{\prime \prime}\left(\mathrm{P}-\mathrm{S}, \mathrm{L}^{\vee}\right)$ is one-dimensional, generated by the current $e^{\vee}$.], $\mathrm{I}[$. The content of (2.13) here is that

$$
\mathrm{Pf} \int_{0}^{1} z^{\lambda_{0}-1}(1-z)^{\lambda_{1}-1} d z \neq 0
$$

The generalization (2.15) of (2.13) can be used to extend this to the case when $\lambda_{0}$ and $\lambda_{1}$, if integers, are $>0$, and $\lambda_{\infty}=1-\lambda_{0}-\lambda_{1}$, if an integer, is $\leq 0$.

Of course, (2.17.1) is easy to deduce from the formula for the B-function in terms of $\Gamma$-functions.

(2. 18) We now assume that each $\alpha_{s}$ is of absolute value $I$, and not equal to $\mathrm{I}$. There is then a horizontal positive Hermitian structure $($,$) on L. Let us choose one.$ One can view $($, ) as a perfect pairing between $\mathrm{L}$ and the complex conjugate local system $\overrightarrow{\mathrm{L}}$. As such, it induces a perfect pairing

$$
\psi_{0}: \mathrm{H}_{c}^{1}(\mathbf{P}-\mathrm{S}, \mathrm{L}) \times_{\mathrm{c}} \mathrm{H}_{c}^{1}(\mathrm{P}-\mathrm{S}, \overline{\mathrm{L}}) \rightarrow \mathrm{H}_{c}^{2}(\mathrm{P}-\mathrm{S}, \mathbf{C})=\mathbf{C}
$$

$((2.3),(2.6 .1))$. The vector space $\mathrm{H}_{c}^{1}(\mathrm{P}-\mathrm{S}, \overline{\mathrm{L}})$ is the complex conjugate of $\mathrm{H}_{c}^{1}(\mathbf{P}-\mathrm{S}, \mathrm{L})$, and $\psi_{0}(u, \bar{v})$ defines a non degenerate skew-Hermitian form $\psi$ on $\mathbf{H}_{c}^{1}(\mathbf{P}-\mathrm{S}, \mathrm{L})$. Anticommutativity of the cup product shows that

$$
\psi_{0}(u, \bar{v})^{-}=-\psi_{0}(v, \bar{u}) \text {, i.c. }
$$

(2.18.1) $\quad \psi(u, v)^{-}=-\psi(v, u)$.

Writing $(u, v):=\frac{-1}{2 \pi i} \psi(u, v)=\frac{-1}{2 \pi i} \psi_{0}(u, \bar{v})$, we have a Hermitian form

(2.18.2) $\quad(u, v)^{-}=(v, u)$.

A section $\omega$ of $j_{*}^{m} \Omega^{1}(\mathrm{~L})$ is said to be of the first kind if $v_{s}(\omega)>-1$ for each $s \in \mathrm{S}$. Those are the forms for which the integral $\int_{P-8} \omega \wedge \bar{\omega}$ is convergent. The integrand is defined as follows: if, locally, $\omega_{i}=e_{i} \cdot \beta_{i}\left(i=1,2, \beta_{i}\right.$ a 1 -form, $e_{i}$ a section of $\left.\mathrm{L}\right)$, then $\omega_{1} \wedge \bar{\omega}_{2}$ is the $(\mathrm{I}, \mathrm{I})$-form $\left(e_{1}, e_{2}\right) \beta_{1} \wedge \beta_{2}$. 
We define $\mathrm{H}^{1,0}(\mathrm{P}-\mathrm{S}, \mathrm{L})$ as the vector space of forms of the first kind in $\Gamma\left(P, j_{*}^{m} \Omega^{1}(L)\right)$, and $H^{0,1}(P-S, L)$ as the complex conjugate of $H^{1,0}(P-S, \bar{L})$. It can be viewed as the space of antiholomorphic $L$-valued forms, whose complex conjugate is of the first kind. Such forms also define cohomology classes.

Proposition (2.19). - If $\omega_{1}$ and $\omega_{2}$ are each either in $\mathrm{H}^{1,0}(\mathrm{P}-\mathrm{S}, \mathrm{L})$ or in $\mathrm{H}^{0,1}(\mathrm{P}-\mathrm{S}, \mathrm{L})$, one has, for the corresponding cohomology classes $\left[\omega_{1}\right]$ and $\left[\omega_{2}\right]$,

$$
\psi\left(\left[\omega_{1}\right],\left[\omega_{2}\right]\right)=\int_{P-B} \omega_{1} \wedge \bar{\omega}_{2} .
$$

Let us assume, for definiteness, that $\omega_{1}$ is in $H^{1,0}(P \ldots-S, L)$. Choose $D_{a},\left(u_{s}\right)_{\imath \in S}$, as in (2.12), and let $\varphi_{8}$ be the characteristic function of $D_{8}$. It follows from (2.12) that the class of $\omega_{1}$ in $H_{c}^{1}(P-S, L)$ is also the class of the compactly supported current $\omega_{1}-\Sigma d\left(\varphi_{s} u_{s}\right)$. Hence (2.3)

$$
\begin{aligned}
\psi\left(\left[\omega_{1}\right],\left[\omega_{2}\right]\right)=\psi_{0}\left(\left[\omega_{1}\right],\left[\bar{\omega}_{2}\right]\right)=\int_{\mathrm{P}-8} & \left(\omega_{1}-\Sigma d\left(\varphi_{s} u_{s}\right)\right) \wedge \bar{\omega}_{2} \\
& =\int_{\mathrm{P}-U \mathrm{D}_{s}} \omega_{1} \wedge \bar{\omega}_{2}+\Sigma \int_{\partial \mathrm{D}_{s}} u_{\mathrm{s}} \bar{\omega}_{2} .
\end{aligned}
$$

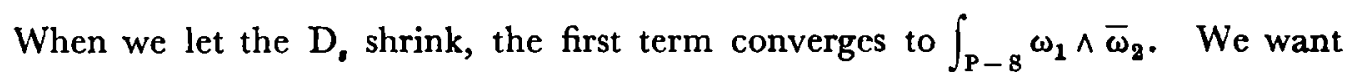
to show that all others tend to 0 . When the radius $r$ of $\mathrm{D}$, tends to zero, the size of $u_{8} \bar{\omega}_{2}$ on $\partial \mathrm{D}_{8}$ is $\mathrm{O}\left\langle r^{v_{s}\left(u_{s}\right)+v_{s}\left(\omega_{2}\right)}\right)$. One has $v_{8}\left(u_{s}\right)=v_{8}\left(\omega_{1}\right)+\mathrm{I}$. The forms $\omega_{1}$ and $\omega_{2}$ being of the first kind, the size is $O\left(r^{\beta}\right)$, with $\beta>-I$. On the other hand, the length of $\partial \mathrm{D}_{\mathrm{s}}$ is $\mathrm{O}(r)$; the boundary term is hence $\mathrm{O}\left(r^{\beta+1}\right)$ and tends to zero.

Proposition (2.20). - Under the assumption (2. I8) that $\left|\alpha_{\mathrm{a}}\right|=\mathrm{I}, \alpha_{\mathrm{s}} \neq \mathrm{I}$ for $s \in \mathrm{S}$, the natural map

$$
\mathrm{H}^{1,0}(\mathrm{P}-\mathrm{S}, \mathrm{L}) \oplus \mathrm{H}^{0,1}(\mathrm{P}-\mathrm{S}, \mathrm{L}) \rightarrow \mathrm{H}^{1}(\mathrm{P}-\mathrm{S}, \mathrm{L})=\mathrm{H}_{c}^{1}(\mathrm{P}-\mathrm{S}, \mathrm{L})
$$

is an isomorphism. The Hermitian form $($,$) is positive definite on \mathrm{H}^{1,0}$, negative definite on $\mathrm{H}^{0,1}$, and the decomposition is orthogonal.

If $\omega$ is in $\mathrm{H}^{1,0}(\mathrm{P}-\mathrm{S}, \mathrm{L}) \quad\left(\right.$ resp. $\left.\mathrm{H}^{0,1}(\mathrm{P}-\mathrm{S}, \mathrm{L})\right)$, the integrand $\frac{-\mathrm{I}}{2 \pi i} \omega \wedge \bar{\omega}$ is $\geq 0$ (resp. $\leq 0$ ), and vanishes only for $\omega=0$. If $\omega_{1}$ and $\omega_{2}$ are one in $H^{1,0}$ and the other in $H^{0,1}$, the integrand $\frac{I}{2 \pi i} \omega_{1} \wedge \bar{\omega}_{2}$ vanishes. This implies that $H^{1,0} \oplus \mathbf{H}^{0,1}$ injects into $\mathrm{H}^{i}(\mathbf{P}-\mathrm{S}, \mathrm{L})$. It remains only to prove the surjectivity of $\mathrm{H}^{1,0} \oplus \mathrm{H}^{0,1} \rightarrow \mathrm{H}^{1}$.

We will check surjectivity by counting dimensions. Let $\mu_{s}$ be the number between $\mathrm{o}$ and $\mathrm{I}$ such that $\alpha_{\mathrm{s}}=\exp \left(2 \pi i u_{\mathrm{s}}\right)$. From the definition, it follows that $\mathrm{H}^{1,0}(\mathrm{P}-\mathrm{S}, \mathrm{L})=\Gamma\left(\mathrm{P}, \Omega^{1}\left(\Sigma \mu_{s} . s\right)(\mathrm{L})\right)$. By $(2.8), \quad \operatorname{deg} \Omega^{1}\left(\Sigma \mu_{z} . s\right)(\mathrm{L})=-2+\Sigma \mu_{\mathrm{s}}$. It is an integer $>-2$ and hence

(2.20.I) $\operatorname{dim} \mathrm{H}^{1,0}(\mathrm{P}-\mathrm{S}, \mathrm{L})=\operatorname{deg} \Omega^{1}\left(\Sigma \mu_{8} . s\right)(\mathrm{L})+\mathrm{I}=-\mathrm{I}+\Sigma_{\mu_{8}}$. 
Let us apply this to $\overline{\mathrm{L}}$. One has to replace $\alpha_{s}$ by $\bar{\alpha}_{s}=\alpha_{s}^{-1}$ and $\mu_{s}$ by $\mathrm{I}-\mu_{s}$. One gets (2.20.2)

$$
\operatorname{dim} \mathrm{H}^{0,1}(\mathbf{P}-\mathrm{S}, \mathrm{L})=-\mathbf{I}+\Sigma\left(\mathbf{I}-\mu_{s}\right) .
$$

The sum of the dimensions is $-2+\Sigma_{I}=\mathrm{N}-2$, as required $(2 \cdot 3 \cdot \mathrm{I})$.

Corollary (2.2x). - Notation being as above $\left(\alpha_{s}=\exp \left(2 \pi i \mu_{s}\right)\right.$ with $\left.0<\mu_{s}<1\right)$, the signature of the Hermitian form (, ) on $\mathrm{H}^{1}(\mathrm{P}-\mathrm{S}, \mathrm{L})$ is $\left(\Sigma \mu_{\mathrm{s}}-\mathrm{I}, \Sigma\left(\mathrm{I}-\mu_{\mathrm{s}}\right)-\mathrm{I}\right)$.

Remark (2.22). - (2.20) is a special case of Hodge theory for the cohomology of a curve with values in a polarized variation of Hodge structures: a local system, provided with an horizontal positive Hermitian structure, can be vicwed as a polarized variation of type $(0,0)$. The artificial counting argument above cnabled us to shortcut the general theory, for which the reader may consult S. Zucker [25].

(2.23) In our applications, the $a_{s}$ will be roots of unity. When this is the case, (2.20) can also be deduced from the Hodge theory of suitable coverings of $P$. Let $X$ be an irreducible abelian covering of $P$, with covering group $G$, ramified only at $S$. If, for definiteness, we take $\mathbf{P}=\mathbf{P}^{1}$ and $\infty \in \mathrm{S}$, this means that the function field $\mathbf{C}(\mathrm{X})$ of $\mathrm{X}$ is a subextension of the extension $\mathbf{C}\left(\mathbf{P}^{1}\right)\left((z-s)^{1 / d} \in \mathbb{S}-\{\infty\}\right)$ of $\mathbf{C}(z)=\mathbf{C}\left(\mathbf{P}^{1}\right)$, for suitable $d$.

Let $\pi$ denote the projection of $X$ onto $P$. The Galois group $G=\operatorname{Aut}(X / P)$ acts (by transport of structure) on $\pi_{*} \mathbf{C}$ (by abuse of notation, we also denote by $\mathbf{G}$ the constant sheaf with fiber $\mathbf{C}$ ), and at a point $z \notin S$, the representation of $G$ on $\left(\pi_{*} \mathbf{C}\right)_{z}=\mathrm{H}^{0}\left(\pi^{-1}(z), \mathbf{C}\right)$ is a regular representation of $\mathrm{G}$. For each character $\chi$ of $\mathrm{G}$, let $L_{x}$ be the subsheaf of $\pi_{*} \mathbf{C}$ on which $G$ acts by $\chi_{\text {. One has }}$

$$
\pi_{*} \mathbf{C}=\bigoplus_{x} \mathrm{~L}_{\mathbf{x}}
$$

and, outside of $\mathrm{S}, \mathrm{L}_{x}$ is a rank one local system.

Let $g_{s} \in \mathrm{G}$ be the natural generator of the inertial (= decomposition) group at $s$ : if $x(t) \quad(0 \leq t \leq \mathrm{I})$ is a path in $\mathrm{X}$, such that $\pi x(t)$ in $\mathrm{P}$ stays near $s$ in $\mathrm{P}-\mathrm{S}$, and turn once around $s$, then $x(\mathrm{I})=g_{8} x(0)$. Let $d_{s}$ be the order of $g_{8}$. If $t$ is a local coordinate centered at $s$, near $s, \mathrm{X}$ is a sum of copies of the Riemann surface of $t^{1 / d_{s}}$.

If $\chi\left(g_{s}\right)=\mathrm{I}, \mathrm{L}_{\chi}$ is a local system at $s$. If $\chi\left(g_{s}\right) \neq \mathrm{I}$, the fibre of $\mathrm{L}_{\chi}$ at $s$ is zero, and the monodromy of $\mathrm{L}_{\chi}$ around $s$ is the $d_{s}$-th root of unity $\chi\left(g_{s}\right)$.

If we take $\mathrm{X}$ to be the largest covering such that $\mathrm{G}$ is killed by $d, \mathrm{G}$ is the abelian group gencrated by the $g_{s}$, with the only relations

$$
g_{s}^{d}=\mathrm{I} \text { and } \Pi g_{s}=\mathrm{I}: \mathrm{G}=(\mathbf{Z} / d)^{\mathrm{s}} /(\mathbf{Z} / d) \text {. }
$$

If $\mathbf{P}=\mathbf{P}^{1}$ and $\infty \in \mathrm{S}, \mathrm{X}$ corresponds to the extension $\mathbf{C}\left(\mathbf{P}^{1}\right)\left((z-s)_{s \in S-\{\infty\}}^{1 / d}\right)$ of $\mathbf{C}\left(\mathbf{P}^{1}\right)$. This shows that any system of roots of unity $\left(\alpha_{s}\right)$, with $\Pi \alpha_{s}=\mathrm{I}$, is of the form $\left(\chi\left(g_{1}\right)\right)_{s \in \mathrm{s}}$ for suitable $\mathrm{X}$ and $\chi$. 
Let $\mathrm{H}^{*}(\mathrm{X}, \mathbf{C})_{X}$ be the subspace of $\mathrm{H}^{*}(\mathrm{X}, \mathbf{C})$ on which $\mathrm{G}$ acts (by transport of structure) as $\chi$. The isomorphism

$$
\mathrm{H}^{*}(\mathrm{X} ; \mathbf{C})=\mathrm{H}^{*}\left(\mathbf{P}, \pi_{*} \mathbf{C}\right)
$$

is compatible with the action of $\mathrm{G}$, hence induces isomorphisms

$$
\mathrm{H}^{*}(\mathrm{X}, \mathbf{G})_{x}=\mathrm{H}^{*}\left(\mathbf{P}, \mathrm{L}_{x}\right) \text {. }
$$

On $\mathbf{P}-\mathrm{S}$, the sheaf $\mathcal{O}\left(\mathrm{L}_{\chi}\right)$ is the direct factor $\left(\pi_{*} \mathcal{O}\right)_{x}$ of $\pi_{*} \mathcal{O}$ consisting of the holomorphic functions on X such that $f\left(g^{-1} x\right)=\chi(g) f(x)$. Similarly, $\Omega^{1}\left(\mathrm{~L}_{\chi}\right)=\left(\pi_{*} \Omega^{1}\right)_{x}$. Let $j^{\prime}$ be the inclusion of $\mathrm{X}-\pi^{-1}(\mathrm{~S})$ in $\mathrm{X}$. On $\mathrm{P}$, one has again

$$
j_{*}^{m}\left(\mathrm{~L}_{\chi}\right)=\left(\pi_{*} j_{*}^{\prime m} \mathcal{O}\right)_{\chi} \quad \text { and }
$$

$$
j_{*}^{m} \Omega^{1}\left(\mathrm{~L}_{x}\right)=\left(\pi_{*} j_{*}^{m} \Omega^{1}\right)_{x} \text {. }
$$

Let us assume $\chi\left(g_{s}\right) \neq \mathrm{I}$ for $s \in \mathrm{S}$, so that $\mathrm{H}^{*}\left(\mathrm{P}, \mathrm{L}_{\chi}\right)=\mathrm{H}_{c}^{*}(\mathrm{P}-\mathrm{S}, \mathrm{L})$. Differential forms of the first kind on $\mathrm{P}-\mathrm{S}$ correspond then, by $(2.23 \cdot 3)$, to the differential forms of the first kind on $\mathrm{X}$, on which $\mathrm{G}$ acts by $\chi$ :

$$
\mathrm{H}^{1,0}\left(\mathrm{P}-\mathrm{S}, \mathrm{L}_{\chi}\right)=\Gamma\left(\mathrm{X}, \Omega^{1}\right)_{\chi}=\mathrm{H}^{1,0}(\mathrm{X})_{\chi},
$$

and $(2.20)$ is the trace on $\mathrm{H}^{1}\left(\mathrm{P}-\mathrm{S}, \mathrm{L}_{\mathrm{X}}\right)=\mathrm{H}^{1}(\mathrm{X}, \mathbf{C})_{x}$ of the Hodge decomposition of $\mathrm{H}^{\mathbf{1}}(\mathrm{X}, \mathbf{G})$.

\section{Reformulation of Picard's theorem}

(3. I) We now let the punctures $s$ move, while the monodromy remains the same. The starting data will be:

$P$ : a complex projective line;

$\mathrm{N}:$ an integer, $\geq 3$;

$\mathrm{S}$ : a set with $\mathrm{N}$ elements, for instance $[\mathrm{I}, \mathrm{N}] \subset \mathbf{N}$;

$\alpha=\left(\alpha_{s}\right)_{s \in \mathrm{S}}$ : a family of complex numbers indexed by $\mathrm{S}$, satisfying $\Pi \alpha_{s}=\mathrm{I}$, and such that none of the $\alpha_{s}$ is I.

We will be mainly interested in the case $\left|\alpha_{s}\right|=\mathrm{I}$.

For any space $X$ mapping to $P^{S}$, we will write $P_{X}$ for the pull back on $X$ of the universal punctured line

$$
\mathrm{P}_{\mathrm{P}^{s}}=\left\{(p, m) \in \mathrm{P} \times \mathrm{P}^{\mathrm{S}} \mid p \notin m(\mathrm{~S})\right\} .
$$

and $\pi$ for the projection $P_{X} \rightarrow X$. Suppose a group $H$ acts frecly on $X$, with quotient $Y$, and that a lifting of the action to $P_{X}$ is given. We will then write $P_{Y}$ for $P_{X} / H$ and $\pi$ for the projection $P_{Y} \rightarrow Y$. The H-equivariant fiber space $P_{X}$ over $X$ is then the pull back of $\mathrm{P}_{\mathrm{Y}}$ over $\mathrm{Y}$.

Let $\mathrm{M} \subset \mathrm{P}^{\mathrm{S}}$ be the space of injective maps $m: \mathrm{S} \rightarrow \mathrm{P}$. Then,

$$
\mathrm{P}_{\mathbf{M}}=\{(p, m) \in \mathbf{P} \times \mathrm{M} \mid p \notin m(\mathrm{~S})\}
$$


is a topologically locally trivial fiber space above $M$. On each fiber $\mathbf{P}_{m}=\mathbf{P}-m(\mathbf{S})$, there is a rank one local system with monodromy $\alpha$, i.e. with monodromy $\alpha_{\text {, }}$ around $m(s)$, for $s \in \mathrm{S}$. However, this local system not being unique up to unique isomorphism, one cannot conclude that on $P_{M}$ there is a unique rank one local system $L$ such that

(3.I.1) For $m$ in $M$, the local system $L_{m}$ induced by $L$ on $P_{m}$ has monodromy $\alpha$. If $L$ satisfies (3.I.I), all local systems satisfying (3.I.I) are of the form $L \otimes \pi^{*} T$, for $\mathrm{T}$ a rank one local system $\mathrm{M}$. We will see in (3.12), (3.13) that there is an L satisfying (3.1. I). If $\mathrm{N}$ were 2, the analogous existence assertion would be false for $\alpha_{8} \neq \pm 1$.

(3.2) Supposc L satisfies (3.1.I). The projection $\pi$ being topologically locally trivial, the $\mathrm{H}^{1}\left(\mathbf{P}_{m}, \mathrm{~L}_{m}\right)\left(=\mathrm{H}_{c}^{1}\left(\mathrm{P}_{m}, \mathrm{~L}_{m}\right)\right.$ by (2.6.I)) organize themselves into a local system $\mathrm{R}^{1} \pi_{*} \mathrm{~L}\left(=\mathrm{R}^{1} \pi_{l} \mathrm{~L}\right)$ on $\mathrm{M}$.

We will be interested mainly in the corresponding flat projective space bundle $\mathrm{B}(\alpha)_{\mathrm{M}}:=\mathrm{PR}^{1} \pi_{*} \mathrm{~L}$, the fiber space with fiber at $m \in \mathrm{M}$ the projective space $\mathrm{PH}^{1}\left(\mathrm{P}_{m}, \mathrm{~L}_{m}\right):=\left(\mathrm{H}^{1}\left(\mathrm{P}_{m}, \mathrm{~L}_{m}\right)-\{0\}\right) / \mathbf{C}^{*}$, and with flat structure, that deduced from $\mathrm{R}^{1} \pi_{*} \mathrm{~L}$. For any vector space $\mathrm{V}$ and non-zero element $v \in \mathrm{V}$, we will denote by $\mathrm{PV}$ the projective space of one-dimensional subspaces of $\mathrm{V}$ and by $\mathrm{Pr}$ the image of 0 in PV.

If $\mathrm{L}^{\prime}=\mathrm{L} \otimes \pi^{*} \mathrm{~T}$ is another local system satisfying (3.I.I),

$$
R^{1} \pi_{*} L^{\prime}=\left(R^{1} \pi_{*} L\right) \otimes T,
$$

and $\mathrm{PR}^{1} \pi_{*} L^{\prime}$ is canonically isomorphic to $\mathrm{PR}^{1} \pi_{*} L$, i.e. the flat projective bundle $B(\alpha)_{M}$ depends only on $\alpha$. Another explanation of the same fact: the automorphisms of a local system $\mathrm{L}_{m}$ on $\mathrm{P}_{m}$ with monodromy $\alpha$ act trivially on $\mathrm{PH}^{1}\left(\mathrm{P}_{m}, \mathrm{~L}_{m}\right)$. Hence, although $\mathrm{L}_{m}$ is unique up to only a non unique isomorphism, $\mathrm{PH}^{1}\left(\mathrm{P}_{m}, \mathrm{~L}_{m}\right)$ is defined up to unique isomorphism.

(3.3) Locally on $M$, the existence of $L$ satisfying (3.1.I) poses no problem: if $\mathrm{U}$ is a contractible neighborhood of $m \in \mathbf{M}$, a local system $\mathrm{L}_{m}$ on $\mathbf{P}_{m}$ with monodromy $\alpha$ extends uniquely to a local system $L_{U}$ on $\pi^{-1}(U)$ and this extension has, fiber by fiber, monodromy $\alpha$. The flat projective space bundle $\mathrm{PR}^{1} \pi_{*} \mathrm{~L}_{\mathrm{U}}$ on $\mathrm{U}$ is independent (up to unique isomorphism) of the choice of $\mathrm{L}_{U}$; hence for variable $\mathrm{U}$, they glue into a flat projective space bundle on the whole of $M$. This enables us to define $B(\alpha)_{M}$ without having to assume the existence of a global $\mathrm{L}$.

For $o \in M$, the flat structure of $B(\alpha)_{M}$ defines an action of $\pi_{1}(M, o)$ by projective transformations on the fibre $B(\alpha)_{0}$. A choice of $L$ enables one to lift this projective representation of $\pi_{1}(M, o)$ on $\operatorname{PH}^{1}\left(P_{o}, L_{o}\right)$ to a linear representation on $\mathbf{H}^{1}\left(\mathbf{P}_{o}, L_{o}\right)$.

(3.4) Fix a system of complex numbers $\left(\mu_{s}\right)_{s \in \mathbb{B}}$ such that $\alpha_{s}=\exp \left(2 \pi i \mu_{s}\right)$, and that $\Sigma \mu_{s}=2$. For each $m \in \mathrm{M}$, there is then up to a factor a unique non-zero section $\omega$ of $\Omega^{1}\left(L_{m}\right)$ on $P_{m}$, meromorphic on $P$, and such that $v_{s}(\omega) \geq-\mu_{s}(s \in S)$ 
(2.12). Its cohomology class is not trivial (2.13). This construction hence defines a section $w_{\mu}(m)$ of the projection $\mathrm{B}(\alpha)_{M} \rightarrow \mathrm{M}$.

The flat structure of the projective space bundle $B(\alpha)$ induces a structure of holomorphic projective space bundle. With respect to it, we have:

\section{Lemma (3.5). - The section $w_{\mu}$ is holomorphic.}

Proof. - The question is local on M. Fix $x \in \mathrm{M}$, and choose on the fibre $\mathrm{P}_{\mathrm{M}}$ a system of cycles $C_{i}$ with coefficient in $L^{\vee}$ giving rise to a basis of $H_{1}\left(P_{m}, L_{m}^{V}\right)$. They have support in some compact $\mathrm{K}$ and, for $\mathrm{U}$ a contractible neighborhood of $m$, small enough so that $\mathrm{K} \times \mathrm{U} \subset \mathrm{P}_{\mathrm{U}}, \mathrm{L} \mid \mathrm{K} \times \mathrm{U}$ is isomorphic to the pull back of $\mathrm{L} \mid \mathrm{K}$ by a unique isomorphism which is the identity above $m$. This enables us, for $m^{\prime} \in \mathrm{U}$, to consider $\mathrm{C}_{i}$ as a cycle with coefficients in $\mathrm{L}^{\vee}$ on $\mathrm{P}_{m^{\prime}}$; and each linear form $\int_{\mathrm{C}_{i}}$ on the $\mathrm{H}^{1}\left(\mathrm{P}_{m^{\prime}}, \mathrm{L}_{m^{\prime}}\right) \quad\left(m^{\prime} \in \mathrm{U}\right)$ is a horizontal linear form on $\mathrm{R}^{1} \pi_{*} \mathrm{~L}$. This provides a horizontal system of projective coordinates on $\mathrm{PR}^{1} \pi_{*} \mathrm{~L}$.

Fix a coordinate $z: \mathbf{P} \rightarrow \mathbf{P}^{1}$, such that, for $m^{\prime}$ in a possibly smaller neighborhood $\mathrm{U}$ of $m, z^{-1}(\infty) \varepsilon: \mathrm{P}_{m^{\prime}}$, and let us trivialize $\mathrm{L}$ along $z^{-1}(\infty)$ by a section $e$. We can takc (cf. (2.12))

$$
\omega=\Pi\left(z-z\left(m^{\prime}(s)\right)\right)^{-u_{s}} \cdot d z . e,
$$

and the projective coordinates of $\omega_{\mu}\left(m^{\prime}\right)$ are the

$$
\int_{\mathfrak{C}_{i}} \Pi\left(z-z\left(m^{\prime}(s)\right)\right)^{-u_{s}} \cdot d z . e .
$$

This is clearly holomorphic in $m^{\prime}$.

Remark (3.6). - A more general method to get horizontal linear forms on $\mathrm{R}^{1} \pi_{*} \mathrm{~L}$ is to start with a $\mathrm{C}^{\infty}$-trivialization of $\left(\mathrm{P}_{\mathrm{U}}, \mathrm{L}\right)$ giving $\varphi_{m^{\prime}}: \mathrm{P}_{m} \stackrel{\sim}{\rightarrow} \mathrm{P}_{m^{\prime}}, \quad \mathrm{L}_{m} \stackrel{\sim}{\rightarrow} \varphi_{m^{\prime}}^{*} \mathrm{~L}_{m^{\prime}}$, and with $L^{\vee}$-valued cycle or current $C$ on $P_{m}$, and to take $\int_{\varphi_{m^{\prime}}(C)}$. For instance, if $\mathrm{C}$ is a path from $m(s)$ to $m(t)$, together with a section $e$ of $\mathrm{L}^{\vee}$ on it, one deforms it with $m^{\prime}$ so that $\mathrm{G}\left(m^{\prime}\right)$ remains a path from $m^{\prime}(s)$ to $m^{\prime}(t)$.

(3.7) The group $G$ of automorphisms of $P$ is isomorphic to $P G L(2)$. Its action on $P$ induces an action on $M \subset P^{S}$, on the space $P_{M}$, and on the flat projective bundle $B(\alpha)_{M}$ on $M$. The section $\omega_{\mu}$ is preserved. The action on $M$ is free. Let $Q=M / G$. The fiber bundle $P_{M}$, the flat bundle $B(\alpha)_{M}$ and $w_{\mu}$ being equivariant, they descend to a fiber bundle $\mathrm{P}_{Q}$, a flat projective space bundle $\mathrm{B}(\alpha)_{Q}$ and a section $w_{\mu}$ of $B(\alpha)_{Q} \rightarrow Q$.

For $\mathbf{P}=: \mathbf{P}^{1}$, and for $a, b, c$ distinct in $\mathrm{S}$, let $\mathrm{M}_{0} \subset \mathrm{M}$ be the space of $m$ such that $m(a)=0, \quad m(b)=1, \quad m(c)=\infty$. One has $\operatorname{PGL}(2) \times \mathrm{M}_{0} \rightarrow \mathrm{M}$. The quotient map hence induces an isomorphism $\mathrm{M}_{0} \stackrel{\sim}{\rightarrow} \mathrm{Q}$, and via this isomorphism, $\mathrm{B}(\alpha)_{Q}$ and $w_{\mu}$ can be identified with the restriction of $\mathrm{B}(\alpha)_{M}$ and $w_{\mu}$ to $\mathrm{M}_{0}$. 
(3.8) Fix a base point $o \in Q$. The section $w_{\mu}$ can then be viewed as a multivalued map from $Q$ to $B(\alpha)_{0}$. More precisely, let $\widetilde{Q}$ be the covering of $Q$ corresponding to the kernel of the monodromy action $\rho$ of $\pi_{1}(Q, o)$ on $B(\alpha)_{0}$, i.e. let $\widetilde{Q}$ be the quotient by $\operatorname{Ker}(\rho)$ of the universal covering of $(Q, 0)$. The pull back of $B(\alpha)_{Q}$ on $\widetilde{Q}$ is $\widetilde{\mathrm{Q}} \times \mathrm{B}(\alpha)_{o}$, and $w_{\mu}$ becomes a $\pi_{1}(\mathrm{Q}, o)$-equivariant map $\widetilde{w}_{\mu}$ from $\widetilde{\mathrm{Q}}$ to $\mathrm{B}(\alpha)_{0}$.

Proposition (3.9). - $\widetilde{w}_{\mu}: \widetilde{\mathrm{Q}} \rightarrow \mathrm{B}(\alpha)_{o}$ is étale.

Proof. - We may take $\mathbf{P}=\mathbf{P}^{1}$ and replace $Q$ by $\mathbf{M}_{0}$, as in (3.7). The problem is local. Take $m \in \mathrm{M}_{0}$, let $e$ be a trivialization of $\mathrm{L}$-near $m$ - on $\mathbf{R}^{+}$near $\infty$. We may take

$$
\omega=\prod_{m(s) \neq \infty}(z-m(s))^{-u_{s}} \cdot d z . e
$$

(cf. (2.8)). Choose cycles $\mathrm{G}_{i}$ as in $(3 \cdot 5 \cdot 4)$. Differentiating in $m$, one has

$$
d_{\mathrm{M}_{0}} \int_{\mathrm{C}_{i}} \omega=\int_{\mathrm{C}_{i}} d_{\mathrm{M}_{0}} \omega \text {, }
$$

with

$$
d_{\mathbb{M}_{0}} \omega=\sum_{, \neq a, b, c} \frac{\mu_{s} d m(s)}{z-m(s)} . \omega .
$$

The spaces $\widetilde{Q}$ and $B(\alpha)_{0}$ have the same dimension $N-3$. The map $\widetilde{w}_{\mu}$ is hence étale at $m$ if and only if $d\left(\widetilde{w}_{\mu}\right)$ is injective, i.e. if and only if for no tangent vector $v \neq 0$ at $m$ is the family $\left(\partial_{v} \int_{C_{i}} \omega\right)_{i}$ proportional to the family of integrals $\left(\int_{C_{i}} \omega\right)_{i}$, i.e. if and only if for no $v$ the cohomology class of $\partial_{v} \omega$ is proportional to that of $\omega$. This means that the cohomology class of any non-trivial linear combination

$$
\eta=a \omega+\sum_{s \neq a, b, c} \frac{b_{s} \omega}{z-m(s)}
$$

should be non-trivial. If $b_{s} \neq 0, \eta$ is of valuation exactly $v_{s}(\omega)-I$ at $s$, and hence $\eta \neq 0$. If $b_{s}=0$, the valuation is $\geq v_{s}$. This shows that $\eta \neq 0$ and that

$$
\sum_{s \in \mathrm{S}} v_{s}(\eta) \geq \sum_{s \in \mathrm{S}} v_{s}(\omega)-(\mathrm{N}-3)=-2-(\mathrm{N}-3)=\mathrm{I}-\mathrm{N} \text {, }
$$

so that the non vanishing of the class of $\eta$ results from (2.14).

(3.10) We will now assume that $\left|\alpha_{s}\right|=I$ and that the numbers $\mu_{8}$ defined by $\alpha_{s}=\exp \left(2 \pi i \mu_{s}\right), \quad 0<\mu<\mathrm{I}$, satisfy $\Sigma_{\mu_{s}}=2$.

Locally on $\mathrm{M}, \mathrm{L}$ admits a horizontal positive definite Hermitian form ( , ), unique up to a positive real factor $(2.18,3 \cdot 3)$. It induces on $R^{1} \pi_{*} L$ a horizontal Hermitian form $()=,\frac{-1}{2 \pi i} \psi(2.18)$, of signature $(\mathrm{I}, \mathrm{N}-3)(2.2 \mathrm{I})$. The vectors on which $($,$) is positive define a horizontal family of complex balls B^{+}(\alpha)_{M} \subset B(\alpha)_{M}$, and the section $w_{\mu}$ is in $\mathrm{B}^{+}(\alpha)_{\mathrm{M}}(2.20)$ (for the meaning of the term "complex ball", see $\S 5$ ). 
The family $\mathrm{B}^{+}(\alpha)_{M}$ is equivariant, hence descends to $\mathrm{B}^{+}(\alpha)_{Q} \subset \mathrm{B}(\alpha)_{Q}$. The étale map of $(3.9)$ is a map

$$
\mathrm{Q} \rightarrow \mathrm{B}^{+}(\alpha)_{0},
$$

equivariant with respect to the action of $\pi_{1}(Q, o)$ on $B^{+}(\alpha)_{0}$. This action is a morphism

$$
\pi_{1}(\mathrm{Q}, \mathrm{o}) \rightarrow \mathrm{PU}\left(\mathrm{H}^{1}\left(\mathrm{P}_{o}, \mathrm{~L}_{o}\right), \frac{-\mathrm{I}}{2 \pi i} \psi\right) .
$$

Notation being as above, our main result is the

\section{Theorem (3.1I). - Assume that}

(INT) The numbers $\mu_{s}$ defined by $\alpha_{s}=\exp \left(2 \pi_{i} \mu_{s}\right), 0<\mu_{s}<\mathrm{I}$, satisfy $\Sigma_{\mu_{s}}=2$ and, for all $s \neq t$ in $\mathrm{S}$ such that $\mu_{s}+\mu_{t}<\mathrm{I},\left(\mathrm{I}-\mu_{s}-\mu_{t}\right)^{-1}$ is an integer.

Then, the image $\Gamma$ of $(3 \cdot 10.2)$ is a lattice in the projective unitary group

$$
\operatorname{PU}\left(\mathrm{H}^{1}\left(\mathbf{P}_{o}, \mathrm{~L}_{o}\right), \frac{-\mathrm{I}}{2 \pi i} \psi\right) \simeq \mathrm{PU}(\mathrm{r}, \mathrm{N}-3) \text {. }
$$

The theorem will be proved in section II, where it is restated as theorem (II.4). The strategy of the proof is presented in the next paragraphs.

In $\S 4$, we define a partial compactification $Q_{8 t}$ of $Q$. We also define a compactification $Q_{s s t}$, with $Q_{s s t} \supset Q_{s t} \supset Q$ and $Q_{s t}-Q_{s t}$ finite. In $\S 8$, we recall a construction of R. H. Fox to define the completion $\widetilde{Q}_{s t}$ (resp. $\widetilde{Q}_{s 8 t}$ ) of $\widetilde{Q}$ over $Q_{s t}$ (resp. $Q_{\text {sat }}$ ). When the condition (INT) is satisfied, one can show that each point $y$ of $Q_{B t}$ admits open neighborhoods $U$ such that the inverse image of $U$ in $\widetilde{Q}$ is a disjoint sum of finite coverings of $U \cap Q$. The completion $\widetilde{Q}_{\text {ot }}$ is then a normal analytic space; it is the normal ramified covering of $Q_{\mathrm{Bt}}$ extending $\widetilde{Q}$.

The results of $\S 6$ allow us, in $\S 8$, to extend $\widetilde{w}_{\mu}$ to a map from $\widetilde{Q}_{\mathrm{Bt}}$ to $\mathrm{B}^{+-}(\alpha)_{0}$. We will again write $\widetilde{w}_{\mu}$ for the extended map. In $\S 9$, we show that the condition INT is tantamount to requiring this extended map to be étale in codimension one. Since the projection $\widetilde{Q}_{s t} \rightarrow Q_{s t}$ is locally (on $\widetilde{Q}_{s t}$ ) finite to one, and $\widetilde{Q}_{s t}$ is a normal analytic space, it follows from $\S 6$ that the extended map $\widetilde{w}_{\mu}: \widetilde{Q}_{s t} \rightarrow \mathbf{B}^{+}(\alpha)_{0}$ is locally finite to onc. By the purity of the branch locus theorem, if $\widetilde{w}_{\mu}$ is étale in codimension one, it is then étale everywhere. Actually, we give two proofs of this fact in $\S 10$, the second proof not requiring the theorem on purity of branch locus. Additional work, relying on the compactness of $Q_{\mathrm{sst}}$, shows that $\widetilde{w}_{\mu}: \widetilde{\mathbf{Q}}_{\mathrm{st}} \rightarrow \mathrm{B}^{+}(\alpha)_{o}$ is a topological covering map. The ball being simply connected, it is an isomorphism. In other words, the inverse of the multivalued map $w_{j}$ is a single valued map $w_{j^{-1}}^{-1}: \mathrm{B}^{+}(x)_{0} \rightarrow \mathrm{Q}_{\mathrm{Bt}}$.

The homcomorphism $\widetilde{w}_{\mu}$ transforms the fibers of the projection $\widetilde{Q}_{s t} \rightarrow Q_{s t}$ into the orbits of $\Gamma$. Those orbits are hence discrete, and $\Gamma$ is a discrete subgroup of the Lie group $\mathrm{PU}\left(1, N^{-} 3\right)$ of isometries of $B^{+}(\alpha)_{0}$. When $Q_{\mathrm{st}}=Q_{\mathrm{st}}$, the 
quotient $\mathrm{B}^{+}(\alpha)_{0} / \Gamma \simeq \mathrm{Q}_{\mathrm{Bt}}$ is compact, and $\Gamma$ is cocompact. In the general case, a local analysis near the cusps $p \in Q_{\text {s8t }}-Q_{\text {st }}$ shows that it has finite covolume.

For $\mathrm{N}=3$, the group $\mathrm{PU}(\mathrm{I}, \mathrm{o})$ is trivial and the statement (3.1I) uninteresting. For $N \geqslant 4$, it is not at once clcar that $\left(\mathrm{I}-\mu_{i}-\mu_{j}\right)^{-1}$ must bc an integer for all $i \neq j$ under condition INT (cf. (I4.2.I)). It is easy to see however that condition INT implies that all $\mu_{s}$ are rational. We show this, even though it is not used in our proof.

Lemma (3.12). - - Assume that card $\mathrm{S} \geqslant 4$. If positive real numbers $\left(\nu_{s}\right)_{s \in \mathrm{s}}$ sum up to 2 and if for each of the pairs of elements $s \neq t$ of $S$ for which $v_{s}+v_{b} \leqslant \mathrm{I}, v_{s}+v_{t}$ is rational, then all $\nu_{\mathrm{s}}$ are rational.

We may and shall assume that $S$ is the set of integer from I to $N, N \geqslant 4$, and that $v_{1} \leqslant \ldots \leqslant v_{x}$.

Case I. $-v_{1}+v_{\mathrm{N}} \geqslant \mathrm{I}$. For any distinct $i, j<\mathrm{N}$ one can find $k<\mathrm{N}$ distinct from $i$ and $j$. One has $v_{k}+v_{\mathrm{N}} \geqslant v_{1}+v_{\mathrm{N}} \geqslant \mathrm{I}$, hence

$$
v_{i}+v_{j} \leqslant 2-\left(v_{k}+v_{\mathrm{N}}\right) \leqslant I
$$

is rational. If three numbers $a, b, c$, are such that $a+b, b+c$ and $c+a$ are rational, they are rational. Applying this to the $v_{i}(i<N)$, we find that they are rational. So is $v_{N}$, because $\Sigma v_{i}=2$.

Case 2. $-v_{1}+v_{N} \leqslant \mathrm{I}$. In this case, for any $i \neq \mathrm{I}, v_{1}+v_{i} \leqslant v_{1}+v_{\mathrm{N}} \leqslant \mathrm{I}$ is rational. Summing, one concludes that $(N-2) v_{1}+\Sigma v_{i}=(N-2) v_{1}+2$ is rational: $v_{1}$ is rational, and so are the $v_{i}(i \neq 1)$.

(3.13) Next, we explain how local systems $L$ with the prescribed monodromy $\alpha$ in the fiber direction can be constructed globally above $M$ on $P_{M}$. Fix thrce distinct elements $a, b, c \in \mathrm{S}$. For each $m$ in $\mathbf{M}$, there is then a unique isomorphism $\mathbf{P} \rightarrow \mathbf{P}^{1}$ mapping $m(a), m(b), m(c)$ respectively to 0 , I, $\infty$. Let $z: \mathbf{P}_{\mathbf{M}} \rightarrow \mathbf{P}^{1}$ be the resulting map. When no ambiguity results, we will write $z(x)$ for $z(x, m)$. Let $\mathrm{A}: \mathbf{M} \rightarrow \mathbf{R}^{+}$ be a large enough continuous function. What is needed is that $[\mathrm{A}(m), \infty[$ be disjoint from $z(m(\mathrm{~S}))$. For any $\mathrm{U} \subset \mathrm{M}$, let $\mathrm{I}_{\mathrm{U}}$ denote the locus of $(x, m) \in \pi^{-1}(\mathrm{U})$ such that $z(x, m) \in\left[\mathrm{A}(m), \infty\left[\right.\right.$. If $\mathrm{U} \subset \mathrm{M}$ is contractible, then $\mathrm{I}_{\mathrm{U}} \subset \mathrm{P}_{\mathrm{M}}$ is too. For $\mathrm{U} \subset \mathrm{M}$ contractible, if $\mathrm{L}$ is a local system on $\pi^{-1}(\mathrm{U})$ with the prescribed monodromy $\alpha$ (cf. (3.3)), then $\mathrm{L}_{\mathrm{U}}$ admits an horizontal section $e_{\mathrm{U}} \neq 0$ on $\mathrm{I}_{\mathrm{U}}$. The pair $\left(\mathrm{L}_{\mathrm{U}}, e_{\mathrm{U}}\right)$ is unique up to unique isomorphism, hence there is no problem in glueing. One gets a global local system $\mathrm{L}$ on $\mathrm{P}_{\mathrm{M}}$, provided with an horizontal section $e$ on $\mathrm{I}_{\mathbf{M}}$.

Let $\mathrm{M}_{0}=\{m \in \mathrm{M} \mid m(a)=0, m(b)=\mathrm{I}, m(c)=\infty\}$ as in (3.7). The product decomposition $\mathrm{PGL}(2) \times \mathrm{M}_{0} \underset{\sim}{\sim} \mathrm{M}$ induces an isomorphism $\mathrm{PGL}(2) \times \mathrm{P}_{\mathrm{M}_{0}} \stackrel{\sim}{\rightarrow} \mathrm{P}_{\mathrm{M}}$; and if one takes $A(m)$ constant on the PGL(2) orbits, so that $I_{M}$ is the pullback of $I_{M_{0}}$, the system $\left(\mathrm{L}_{M}, e\right)$ is the pull back of its restriction to $\mathrm{P}_{M_{\bullet}}$. 
(3.14) We give two other descriptions of the same $L$. Fix numbers $\mu_{s}$ such that $\alpha_{s}=\exp \left(2 \pi i \mu_{s}\right)$. Let $L_{\mu}$ be the subsheaf of $\mathcal{O}$ (cf. $\left.\S 2\right)$ consisting of those functions which are constant multiples of $\prod_{s \neq c}(z-z(m(s)))^{\mu_{s}}$. This function is multivalued but the ratio of two determinations is a constant, so that the definition of $L_{\mu}$ makes sense. If $\mu^{\prime}$ is another choice of $\mu$, with $\alpha_{s}=\exp \left(2 \pi i \mu_{s}^{\prime}\right)$, the multiplication by the univalued function $\prod_{s \neq c}(z-z(m(s)))^{\mu_{s}^{\prime}-\mu_{s}}$ is an isomorphism of $\mathrm{L}_{\mu}$ with $\mathrm{L}_{\mu^{\prime}}$.

The principal determination of the multivalued function $(z-z(m(s)))^{\mu_{s}}$ on $\mathrm{I}_{\mathrm{MI}}$ is defined as $\exp \left(\mu_{s} \log (z-z(m(s)))\right)$ with $|\arg \log (z-z(m(s)))|<\pi i$ for $z$ real and large enough. This provides a section of $L_{\mu}$ on $I_{M}$. One easily checks that $L_{\mu}$ has the monodromy $\alpha$ on each fibre.

An identical but more algebraic description of $L_{\mu}$ is: the local system of horizontal sections of $\mathcal{O}$, provided with the connection $\nabla_{\mu}$ for which

$$
f^{-1} \nabla_{\mu} f=\frac{d f}{f}-\sum_{s \neq c} \frac{\mu_{s}(d z-d z(m(s)))}{z-z(m(s))} .
$$

(3.15) We close section 3 with some remarks about liftings of the projective representation

$$
\theta: \pi_{1}(\mathrm{Q}, 0) \rightarrow \mathrm{PU}\left(\mathrm{H}^{\mathrm{1}}\left(\mathrm{P}_{0}, \mathrm{~L}_{0}\right), \frac{-\mathrm{I}}{2 \pi i} \psi\right)
$$

to a linear representation.

As in (3.7) and (3.13), fix thrce elements $a, b, c$ in $\mathrm{S}$ and denote by $\mathrm{M}_{0}$ the subset of $\mathrm{M}$ with $m(a)=0, m(b)=\mathrm{I}$ and $m(c)=\infty$. Let $\mathrm{M}(c)$ denote the subset of $m \in \mathrm{M}$ with $m(c)=\infty$, and let $\mathbf{B}$ denote the stabilizer of $\infty$ in $\operatorname{PGL}(2)$. One has $\mathrm{M}_{0} \stackrel{\sim}{\rightarrow}$ and

$$
\mathrm{B} \times \mathrm{M}_{0} \stackrel{\sim}{\rightarrow} \mathrm{M}(c), \quad \mathrm{PGL}(2) \times \mathrm{M}_{0} \stackrel{\sim}{\rightarrow} \mathrm{M} .
$$

As is well known,

and

$$
\pi_{1}(\mathrm{PGL}(2))=\pi_{1}(\mathrm{PU}(2))=\pi_{1}(\mathrm{SU}(2) / \pm \mathrm{I})=\mathbf{Z} / 2
$$

so that

(3.15.1)

$$
\pi_{\mathbf{1}}(\mathbf{B})=\pi_{1}\left(\mathbf{C} \times \mathbf{C}^{*}\right)=\mathbf{Z} \text {, }
$$

$$
\pi_{1}(\mathrm{M}) \approx \pi_{1}(\mathrm{Q}) \times \mathbf{Z} / \mathbf{2},
$$$$
\pi_{1}(\mathrm{M}(c)) \approx \pi_{1}(\mathrm{Q}) \times \mathbf{Z} .
$$

Both those isomorphisms depend on the choices of $a, b, c$.

For $\mathrm{X}$ any of $\mathrm{Q}, \mathrm{M}(c)$ or $\mathrm{M}$, each choice of a local system $\mathrm{L}$ on $\mathrm{P}_{\mathrm{X}}$ with fiberwise monodromy $a$ leads to a lifting

$$
\theta^{\prime}: \pi_{1}(\mathrm{X}, o) \rightarrow \mathrm{GL}\left(\mathrm{H}^{1}\left(\mathrm{P}_{o}, \mathrm{~L}_{0}\right)\right)
$$


of $\theta$. If $L$ carries a unitary structure, $\theta^{\prime}$ has values in $U\left(H^{1}\left(P_{o}, L_{o}\right), \frac{-I}{2 \pi i} \psi\right)$. For instance, the $L$ of (3.12) carries a unique unitary structure for which $e$ is of length one, and its restriction to $\mathrm{M}_{0} \stackrel{\sim}{\rightarrow} Q$ provides a lifting $\theta^{\prime}: \pi_{1}(Q, o) \rightarrow \mathrm{U}\left(\mathrm{H}^{1}\left(\mathrm{P}_{0}, \mathrm{~L}_{0}\right), \frac{-1}{2 \pi i} \psi\right)$. The local system $L$ itself provides a lifting $\theta^{\prime}: \pi_{1}(M, o) \rightarrow U\left(H^{1}\left(P_{o}, L_{o}\right), \frac{-1}{2 \pi i}\right)$ which is trivial on the second factor of the decomposition (3.15.I).

Let $\mathrm{L}(c)$ denote the local system on $\mathrm{P}_{\mathbf{M}(c)}$ which is given by the subsheaf of $\mathcal{O}$ consisting of those functions which are constant multiples of

$$
\prod_{s \neq c}(z-m(s))^{\mu_{s}}
$$

Here, $z$ is a fixed coordinate on $\mathbf{P}$ composed with $\mathrm{P} \times \mathrm{M}(c) \rightarrow \mathrm{P}$ and not as in (3. I2) and (3. I3). The local system $\mathrm{L}(c)$ provides a lifting $\theta^{\prime}: \pi_{1}(\mathrm{M}(c)) \rightarrow \mathrm{U}\left(\mathrm{H}^{1}\left(\mathrm{P}_{o}, \mathrm{~L}_{o}\right), \frac{-\mathrm{I}}{2 \pi i} \psi\right)$
of $\theta$.

Inasmuch as $M(c)$ is the space of distinct $(N-I)$-uples of points in the plane $(N=$ card $S), \pi_{1}(\mathbf{M}(c))$ is by definition the colored braid group on $(N-1)$ strands. For any $s, t \in \mathrm{S}-c$, let $\gamma_{s t}$ in $\pi_{1}(\mathrm{M}(c))$ be a path in which $t$ comes near $s$, makes a positive turn around $s$ and comes back to its original position. The colored braid group is generated by such elements. The lifting $\theta^{\prime}$ provided by $\mathrm{L}(c)$ has the virtue that each $\theta^{\prime}\left(\gamma_{s, l}\right)$ is a pseudo-reflection (i.e. $\theta^{\prime}\left(\gamma_{s, t}\right)-1$ is of rank one)-cf. (9.1), (12.3).

\section{The compactified quotient space $Q_{\text {sst }}$}

(4.0) As in section 3 , we fix a complex projective line $P$, an integer $N \geqslant 3$ and a finite set $\mathrm{S}$ with $\mathrm{N}$ elements. We further fix a family $\mu=\left(\mu_{s}\right)_{s \in \mathrm{S}}$ of real numbers with $0<\mu_{s}<I$ and

$$
\Sigma \mu_{s}=2 \text {. }
$$

The complex numbers $\alpha_{s}:=\exp \left(2 \pi i \mu_{s}\right)$ satisfy $\left|\alpha_{s}\right|=\mathrm{I}, \alpha_{s} \neq \mathrm{I}$ and $\Pi \alpha_{8}=\mathrm{I}$.

As in section $3, \mathrm{M} \subset \mathrm{P}^{\mathrm{s}}$ is the space of injective maps $y: \mathrm{M} \rightarrow \mathrm{P}$. We denote by PGL(2) the group of automorphisms of $P$.

These conventions will hold throughout this section as well as in sections 6 to 12 . In this section, from (4.2) on, $P$ will be the standard projective line $\mathbf{P}:=\mathbf{P}^{1}=\mathbf{C} \cup\{\infty\}$ and $\mathrm{M}$ the space of S-uples of distinct points of the Riemann sphere. This is no loss of generality.

(4. I) The group PGL(2) operates diagonally on $\mathrm{P}^{\mathrm{s}}$. We shall define a compactification of the quotient space $Q:=P G L(2) \backslash M$. The definition is taken from D. Mum- 
ford's theory of quotient varieties of reductive groups (cf. (4.6)). The account here is self-contained.

A point $y \in \mathrm{P}^{\mathrm{S}}$ is called $\mu$-stable (resp. semi-stable) if and only if for all $z \in \mathrm{P}$, (4.I.I)

$$
\sum_{y(s)=z} \mu_{s}<1 \quad(\text { resp. } \leq 1)
$$

The set of all $\mu$-stable points (resp. $\mu$-semi-stable points) is denoted

$$
\mathbf{M}_{\mathrm{st}} \text { (resp. } \mathrm{M}_{\mathrm{sst}} \text { ) }
$$

and we set $M_{\text {ousp }}=M_{\text {sit }}-M_{\text {st }}$.

For each partition $\left\{\mathrm{S}_{1}, \mathrm{~S}_{2}\right\}$ of $\mathrm{S}$ with $\sum_{s \in \mathrm{B}_{i}} \mu_{s}=\mathrm{I} \quad(i=\mathrm{r}, 2)$, the points $y$ in $\mathrm{P}^{\mathrm{s}}$ for which $y\left(\mathbf{S}_{1}\right) \cap y\left(\mathbf{S}_{2}\right)=\varnothing$ and $y$ constant on $\mathrm{S}_{1}$ or $\mathbf{S}_{2}$ are in $\mathbf{M}_{\text {ousp }}$. All points in $\mathrm{M}_{\text {cusp }}$ are obtained in this way, each from a unique partition.

On $M_{s s t}$ we define a relation $\mathscr{R}$ via

$$
y \equiv y^{\prime}(\mathscr{R}) \text { if and only if either }
$$

a) $y, y^{\prime} \in \mathrm{M}_{\mathrm{at}}$ and $y^{\prime} \in \mathrm{PGL}(2) y$ or

b) $y, y^{\prime} \in \mathrm{M}_{\text {cusp }}$ and the partitions of $\mathrm{S}$ corresponding to $y$ and $y^{\prime}$ coincide.

It is clear that $\mathscr{R}$ is an equivalence relation. Set

$$
Q_{\text {sst }}=M_{s s t} / \mathscr{R}, \quad Q_{\text {st }}=M_{s t} / \mathscr{R}, \quad Q_{\text {ousp }}=M_{\text {cusp }} / \mathscr{R}
$$

each with its quotient topology. The clements of $Q_{\text {cusp }}$ are uniquely determined by their partitions. Thus $Q_{\text {ousp }}$ is a finite set.

Example (4.2). - The cross-ratio $c\left(z_{1}, z_{2}, z_{3}, z_{4}\right)=\frac{z_{1}-z_{3}}{z_{3}-z_{2}}: \frac{z_{1}-z_{4}}{z_{4}-z_{2}}$ of four points in $\mathbf{P}:=\mathbf{P}^{1}$ is defined and is a continuous map into $\mathbf{P}^{1}$ as long as no three of $z_{1}$, $z_{2}, z_{3}, z_{4}$ are equal. When two of the $z_{i}$ are equal, the cross-ratio takes one of the values $0, I, \infty$, the value depending only on the partition of $\{1,2,3,4\}$ consisting of the subset $\{i, j\}$ with $z_{i}=z_{j}$ and its complement. Suppose $S=\{1,2,3,4\}$ and $\mu_{s}=\frac{I}{2}$ for all $s \in \mathrm{S}$. One can show that the cross-ratio $\operatorname{map} \mathbf{M}_{s s t} \rightarrow \mathbf{P}^{1}$ descends to $Q_{\text {sst }}$ and yields a homeomorphism onto the projective line.

(4.3) Let $\mathrm{M}^{\prime}$ be the space of $y \in \mathbf{P}^{\mathrm{s}}$ such that $y(\mathrm{~S})$ has at least three distinct points, and let $Q^{\prime}=\operatorname{PGL}(2) \backslash M^{\prime}$ be the corresponding quotient orbit space. Fix $a, b, c$ in $\mathrm{S}$, let $\mathrm{U}$ be the set of all $y \in \mathrm{M}^{\prime}$ taking distinct values on $a, b, c$, and let $\mathrm{U}_{\mathbf{0}}$ be the subset of all $y \in \mathrm{M}$ for which $y(a)=0, y(b)=\mathrm{I}, y(c)=\infty$. Then $\mathrm{U}$ is in $\mathrm{M}$, stable under the action of $\operatorname{PGL}(2)$, and $\operatorname{PGL}(2) \times \mathrm{U}_{0} \stackrel{\sim}{\rightarrow} \mathrm{U}$. Hence

$$
\mathrm{U}_{0} \stackrel{\sim}{\rightarrow} \mathrm{PGL}(2) \backslash \mathrm{U} \hookrightarrow \mathrm{Q}^{\prime} \text {. }
$$

As one varies $a, b, c$, the images of $\mathrm{U}_{0}$ in $Q^{\prime}$ cover $Q^{\prime}$. Consequently $Q^{\prime}$ is a manifold. However, this manifold is not Hausdorff if card $S>3$. For card $S=4$ (cf. (4.2)) 
one gets the projective line with $o, I$ and $\infty$ doubled. One infers from (4.I.I) that $M_{s t} \subset M^{\prime}$. The quotient $Q_{s t}$ being open in $Q^{\prime}$ is a manifold. The following Lemma implies that it is Hausdorff.

Lemma (4 3). - $\mathrm{Q}_{\mathrm{Bst}}$ is Hausdorff.

Proof. - Let $\mathbf{F}$ denote the set of injective maps of $\{1,2,3,4\}$ into S. For any $f \in \mathrm{F}$, let $\mathrm{M}_{f}$ denote the set of all $y \in \mathrm{M}_{88 \mathrm{t}}$ such that $\operatorname{card}(y f)^{-1}(z) \leq 2$ for all $z \in \mathbf{P}$ and additionally, if $y \in \mathrm{M}_{\text {cusp }}, \operatorname{card} f^{-1}\left(\mathrm{~S}_{i}\right)=2$ for $i=1,2$ where $\left\{\mathrm{S}_{1}, \mathrm{~S}_{2}\right\}$ is the partition of $\mathrm{S}$ corresponding to $y$. The additional condition guarantees that $\mathrm{M}_{f} \cap \mathrm{M}_{\text {cusp }}$ as well as $\mathbf{M}_{f} \cap \mathbf{M}_{\mathrm{st}}$ is $\mathscr{R}$-saturated.

For any $f$ in $\mathbf{F}$, define $c_{f}: \mathbf{M}_{l} \rightarrow \mathbf{P}$ via the cross-ratio:

$$
c_{f}(y)=c(y f(\mathrm{I}), y f(2), y f(3), y f(4)) \text {; }
$$

the function $c_{f}$ is constant on $\mathscr{R}$-equivalence classes of the $\mathscr{R}$-saturated set $\mathrm{M}_{f}$. Inasmuch as $c_{f}$ is continuous on $\mathrm{M}_{f}$, it descends to a continuous function on $\mathrm{M}_{f} / \mathscr{R}$. To prove the lemma, it suffices to prove:

(4.3. I) For any $y \neq y^{\prime} \bmod \mathscr{R}$, there is an $f \in \mathrm{F}$ such that $y$ and $y^{\prime}$ are in $\mathbf{M}_{j}$, and $c_{i}(y) \neq c_{f}\left(y^{\prime}\right)$.

One is free to replace $y$ and $y^{\prime}$ by $y_{1}$ and $y_{1}^{\prime}$ with $y \equiv y_{1}(\mathscr{R}), y^{\prime} \equiv y_{1}^{\prime}(\mathscr{R})$.

To cach $y$ in $\mathrm{M}_{\mathrm{8st}}$ we attach a partition of $\mathrm{S}$ as follows: for $y \in \mathrm{M}_{\text {ousp }}$, attach the partition defined in $(4 . \mathrm{I})$; for $y \in \mathrm{M}_{\mathrm{st}}$, attach the partition $\mathrm{T}=\left\{\mathrm{C}_{1}, \ldots, \mathrm{C}_{\mathrm{r}}\right\}$ such that $y(s)=y(t)$ if and only if $s$ and $t$ lie in the same coset of T. If $y \equiv y_{1}(\mathscr{R})$, the same partition is attached to $y$ and $y_{1}$. In the proof of $(4 \cdot 3 \cdot 1)$, we may and shall assume that if $y$ or $y^{\prime}$ is in $\mathbf{M}_{\text {cusp }}$, then it is constant on the cosets of its partition.

We first treat the case that $y$ and $y^{\prime}$ are in $\mathrm{M}_{\text {cusp }}$. Let $\left\{\mathrm{S}_{1}, \mathrm{~S}_{2}\right\}$ and $\left\{\mathrm{S}_{1}^{\prime}, \mathrm{S}_{2}^{\prime}\right\}$ be their corresponding partitions of $S$. Since $y \neq y^{\prime} \bmod \mathscr{R}, S_{i} \cap S_{j}^{\prime}$ is not empty for $i, j \in\{\mathrm{I}, 2\}$; otherwise, if say $\mathrm{S}_{1} \cap \mathrm{S}_{2}^{\prime}=\varnothing$, we would have $\mathrm{S}_{1} \subset \mathrm{S}_{1}^{\prime}, \mathrm{S}_{1} \neq \mathrm{S}_{1}^{\prime}$ and

$$
\sum_{s \in S_{i}-S_{1}} \mu_{s}=0
$$

contradicting $0<\mu_{s}$ for each $s \in \mathrm{S}$. Choose $f$ so that $\operatorname{card} f^{-1}\left(\mathrm{~S}_{i} \cap \mathrm{S}_{j}\right)=\mathrm{I}$ for each choice of $i, j \in\{1,2\}$. Then for suitable choice of $f$ we have

$$
\begin{aligned}
& y f(1), y f(2), y f(3), y f(4)=a, a, b, b, \\
& y^{\prime} f(1), y^{\prime} f(2), y^{\prime} f(3), y^{\prime} f(4)=a^{\prime}, b^{\prime}, a^{\prime}, b^{\prime},
\end{aligned}
$$

with $a \neq b, a^{\prime} \neq b^{\prime}$. Hence

$$
c_{f}(y)=\mathrm{I}, \quad c_{f}\left(y^{\prime}\right)=\mathrm{o} .
$$

Suppose next that $y \in \mathrm{M}_{\text {cusp }}$ and $y^{\prime} \in \mathrm{M}_{\mathrm{st}}$. Let $\mathrm{T}=\{\mathrm{C}\}$ be the partition of $\mathrm{S}$ corresponding to $y^{\prime}$ by (4.2). Each coset in the partition of $\mathrm{S}$ corresponding to $y$ must meet at least two distinct cosets of $\mathrm{T}$. We can thus choose an $f \in \mathrm{F}$ so that 


$$
\begin{aligned}
& y f(\mathbf{1}), y f(2), y f(3), y f(4)=a, a, b, b, \\
& y^{\prime} f(\mathrm{I}), y^{\prime} f(2), y^{\prime} f(3), y^{\prime} f(4)=a^{\prime}, b^{\prime}, c, d,
\end{aligned}
$$

with $a \neq b, a^{\prime} \neq b^{\prime}$, and $c \neq d$. It follows at once that $y$ and $y^{\prime}$ are in $\mathrm{M}_{f}, c_{f}(y)=\mathrm{I}$, $c_{f}\left(y^{\prime}\right) \neq \mathrm{r}$.

It remains only to consider the case where $y$ and $y^{\prime}$ are in $M_{s t}$; let $T$ and $T^{\prime}$ be the corresponding partitions of $\mathbf{S}$. If $\mathbf{T}=\mathbf{T}^{\prime}$, then for any $f \in \mathbf{F}, y \in \mathbf{M}_{f}$ if and only if $y^{\prime} \in \mathrm{M}_{f}$. Replacing $y^{\prime}$ by an clement in its PGL(2)-orbit, we can assume that there are three distinct cosets $\mathrm{C}_{1}, \mathrm{C}_{2}, \mathrm{C}_{3}$ of $\mathrm{T}$ with $y(s)=y^{\prime}(s)$ for $s \in \mathrm{C}_{1} \cup \mathrm{C}_{2} \cup \mathrm{C}_{3}$. If $c_{f}(y)=c_{f}\left(y^{\prime}\right)$ for all $f$ with $y \in \mathrm{M}_{1}$, then clearly $y=y^{\prime}$. One is thus reduced to the case where $T \neq T^{\prime}$. Then there is a $C_{1} \in T$ and $C_{1}^{\prime} \in T^{\prime}$ such that $G_{1} \cap C_{1}^{\prime}$ is not empty and $\mathrm{C}_{1} \neq \mathrm{C}_{1}^{\prime}$.

Either $\mathbf{G}_{1}-\mathrm{C}_{1}^{\prime}$ or $\mathrm{C}_{1}^{\prime}-\mathrm{C}_{1}$ is not empty-say $\mathrm{C}_{1}^{\prime}-\mathrm{C}_{1} \neq \varnothing$. Choose $\mathrm{C}_{2} \in \mathbf{T}$ so that $\mathrm{C}_{1}^{\prime} \cap \mathrm{C}_{2} \neq \varnothing$. Choose $\mathrm{C}_{2}^{\prime} \in \mathrm{T}^{\prime}$ with $\mathrm{C}_{2}^{\prime} \neq \mathrm{C}_{1}^{\prime}$. Next choose $\mathrm{G}_{i} \in \mathrm{T}$ so that $\mathrm{C}_{2}^{\prime} \cap \mathrm{C}_{i} \neq \varnothing$. Then $\mathrm{C}_{1}^{\prime} \cup \mathrm{C}_{i} \neq \mathrm{S}$; otherwise, since $y^{\prime}$ and $y$ are in $\mathrm{M}_{\mathrm{st}}$,

$$
2 \leq \sum_{s \in \mathrm{C}_{i}^{\prime}} \mu_{s}+\sum_{s \in \mathrm{C}_{i}} \mu_{s}<\mathrm{I}+\mathrm{I} \text {. }
$$

Choose $f:\{1,2,3,4\} \rightarrow \mathrm{S}$ with

$$
f(\mathrm{I}) \in \mathrm{C}_{1}^{\prime} \cap \mathrm{C}_{1}, \quad f(2) \in \mathrm{G}_{1}^{\prime} \cap \mathrm{C}_{2}, \quad f(3) \in \mathrm{G}_{2}^{\prime} \cap \mathrm{G}_{i}, \quad f(4) \notin \mathrm{C}_{1}^{\prime} \cup \mathrm{G}_{i} .
$$

Any such $f$ is injective, and

$$
\begin{aligned}
& y f(\mathrm{r}), y f(2), y f(3), y f(4)=a, b, c, d, \quad \text { with } a \neq b, c \neq d \\
& y^{\prime} f(\mathrm{I}), y^{\prime} f(2), y^{\prime} f(3), y^{\prime} f(4)=a^{\prime}, a^{\prime}, b^{\prime}, c^{\prime} \quad \text { with } a^{\prime} \neq b^{\prime}, a^{\prime} \neq c^{\prime} .
\end{aligned}
$$

Hence $c_{f}\left(y^{\prime}\right)=\mathrm{I}, c_{y}(y) \neq \mathrm{I}$. This complctes the proof:

Lemma (4.4.r). - Fix a Riemannian metric on $\mathbf{P}$. Let $\mathscr{G}$ be the set of all subsets $\mathrm{T}$ of $\mathrm{S}$ with $\sum_{s \in \mathbf{T}} \mu_{s}>\mathrm{I}$. For $y \in \mathbf{P}^{\mathrm{S}}$, define

$$
d(y)=\inf _{\mathrm{T} \in \boldsymbol{\sigma}} \operatorname{diam} y(\mathrm{~T})
$$

and

$$
a(y)=\sup _{g \in \mathrm{P}(\mathrm{L}(2)} d(g y) \text {. }
$$

Then there is $a>0$ such that $a(y) \geq a$ for all $y \in \mathbf{M}_{\mathrm{sqt}}$.

Proof. - - The validity of the lemma is independent of the chosen metric. We will use the Fubini metric (for which $\mathbf{P}$ is isometric to the sphere of radius $I$ ) and prove the lemma with $a=\pi / 4$.

If $y \in \mathrm{M}_{\text {eusp }}$ corresponds to the partition $\left\{\mathrm{S}_{1}, \mathrm{~S}_{2}\right\}$ of $\mathrm{S}$, with $y$ constant on $\mathrm{S}_{1}$, let us take $g$ to fix $y\left(\mathrm{~S}_{1}\right)$ and the antipodal point $u$ of $\mathrm{P}$ and to carry the complement of the $\varepsilon$-neighborhood of $y\left(\mathrm{~S}_{1}\right)$ into the $\varepsilon$-neighborhood of $u$. Taking $\varepsilon \rightarrow 0$, one gets $d(y)=\pi$. 
For any $\varepsilon$, there is a compact $\mathrm{K} \subset \mathrm{PGL}(2)$ such that for $g \notin \mathrm{K}, g$ maps the complement of the $\varepsilon$-neighborhood of some point $u$ into the $\varepsilon$-neighborhood of some point $v$. If $y \in \mathrm{M}_{\mathrm{st}}$ and if $\varepsilon$ is small enough, then, for all $u,\{s \in \mathrm{S} \mid \operatorname{dist}(\mathcal{y}(s), u)>\varepsilon\}$ is in $\tilde{F}$. This implies $d(g y) \rightarrow 0$ for $g \rightarrow \infty$ in PGL(2). The sup defining $a(y)$ is hence attained. Fix $y \in \mathrm{M}_{\mathrm{at}}$ with $a(y)=d(y)$. We have to prove $d(y) \geq a=\pi / 4$.

Suppose to the contrary that $d(y)<a$. Fix $\mathrm{T}_{1} \in \mathscr{E}$ with $\operatorname{diam} y\left(\mathrm{~T}_{1}\right)<a$ and $t \in \mathrm{T}_{1}$. For any $\mathrm{T}_{1}, \mathrm{~T}_{2}$ in $\mathscr{E}, \mathrm{T}_{1} \cap \mathrm{T}_{2} \neq \varnothing$. If $\mathrm{S}^{\prime}$ is the union of all $\mathrm{T} \in \mathscr{G}$ with $\operatorname{diam} y(\mathrm{~T})<a, y\left(\mathrm{~S}^{\prime}\right)$ is hence contained in a half-sphere of radius $r<2 a=\pi / 2$ with center $y(t)$. In particular, $y\left(\mathrm{~S}^{\prime}\right)$ is contained in an open halfsphere. Identify $\mathrm{P}$ with the Riemann sphere $\sigma \cup \infty$, with the unit disc the halfsphere, in such a way that $y\left(\mathbf{S}^{\prime}\right)$ lies in $\mathrm{D}=\{z|| z \mid \leq b\}, \quad b<\mathrm{I}$. Take for $g$ the multiplication by $\mathrm{I}+\varepsilon, \varepsilon>\mathbf{0}$. For $\varepsilon$ small enough (relative to $b$ ), distances in $\mathrm{D}$ are increased by $g$. For any $\mathrm{T} \in \mathbb{E}$ with $\operatorname{diam} y(T)<a$, one hence has $\operatorname{diam} g y(\mathrm{~T})>\operatorname{diam} y(\mathrm{~T})$. For $\mathrm{T} \in \mathscr{E}$ with $\operatorname{diam} y(\mathrm{~T}) \geq a, \operatorname{diam} y(\mathrm{~T})$ can decrease, but not much for $\varepsilon$ small, so that $d(g y)>d(y)$. This contradicts the choice of $y$.

Lemma (4.4.2). - $Q_{88 \mathrm{t}}$ is compact.

Fix $a>0$ as in $(4 \cdot 4 \cdot 1)$, and define $M^{\prime} \subset \mathbf{P}^{s}$ to be the set of $y$ such that $\operatorname{diam} y(\mathrm{~T}) \geq a$ for each $\mathrm{T} \subset \mathrm{S}$ with $\sum_{a \in \mathrm{T}} \mu_{s}>\mathrm{I}$. The subset $\mathrm{M}^{\prime}$ of $\mathbf{P}^{\mathrm{S}}$ is closed, hence compact. It is contained in $M_{8 \mathrm{st}}$ and, by (4.4.I), it maps onto $Q_{8 \mathrm{st}}$. Compactness follows.

(4.5) By contrast with $Q_{\mathrm{st}}$ which is a manifold, the points of $Q_{\text {ousp }}$ may be singular.

Let $\psi$ denote the natural projection of $M_{s s t}$ onto $Q_{8 s t}$. Let $\left\{S_{1}, S_{1}^{\prime}\right\}$ be the partition corresponding to a point $y_{*} \in \mathrm{M}_{\text {ousp }}$. We shall describe a neighborhood of $\psi\left(y_{*}\right)$ in $Q_{* s t}$. We can assume that $y_{*}(s)=0$ for all $s \in \mathrm{S}_{1}$ and $y_{*}(s)=\infty$ for all $s \in \mathrm{S}_{1}^{\prime}$. Fix elements $a \in \mathrm{S}_{1}$ and $b \in \mathrm{S}_{1}^{\prime}$, and define $\mathrm{V}$ as the set of all $y \in \mathrm{M}_{\mathrm{sst}}$ such that $y(a)=0, y(b)=\infty$ and

$$
\sup _{s \in \mathrm{S}_{1}}|y(s)|<\inf _{\boldsymbol{s} \in \mathrm{S}_{\mathbf{i}}}|y(s)| \text {. }
$$

Then, $\dot{\psi}(\mathrm{V})$ is an open ncighborhood of $\dot{\psi}\left(y_{*}\right)$ in $Q_{\mathrm{sgt}}$ and is the quotient of $\mathrm{V}$ by the equivalence relation on $\mathrm{V}$ induced by $\mathscr{R}$. The punctured neighborhood $\psi(\mathrm{V})-\psi\left(y_{0}\right)$ is the quotient of $\mathrm{V}-\psi^{-1} \psi\left(y_{*}\right)$ by the stabilizer in $\mathrm{PGL}(2)$ of the points 0 and $\infty$ : $\psi(V)-\psi\left(y_{*}\right) \cong\left(\mathrm{V}-\dot{\psi}^{-1} \dot{\psi}\left(y_{*}\right)\right) / \mathbf{C}^{*}$.

Set $\mathrm{S}_{2}=\mathrm{S}_{1}-\{a\}, \mathrm{S}_{2}^{\prime}=\mathrm{S}_{1}^{\prime}-\{b\}, \quad m=\operatorname{card}\left(\mathrm{S}_{2}\right), m^{\prime}=\operatorname{card}\left(\mathrm{S}_{2}^{\prime}\right)$ and consider the composite map

$$
\begin{aligned}
& \beta: \mathrm{V} \rightarrow \mathbf{C}^{\mathbf{S}_{\mathbf{s}}} \times \mathbf{C}^{\mathrm{S}_{\mathbf{1}}^{\prime}}=\mathbf{C}^{m} \times \mathbf{C}^{m^{\prime}} \rightarrow \mathbf{C}^{m m^{\prime}}
\end{aligned}
$$

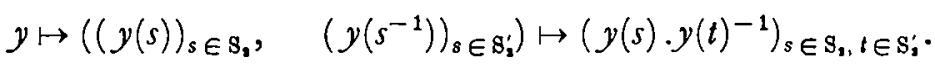


The map $\beta$ descends to an embedding of $\psi(V)$ inro $\mathbf{C}^{m m^{\prime}}$, whose image is the intersection of the cone over the Veronese embedding of $\mathbf{P}^{m-1} \times \mathbf{P}^{m^{\prime}-1}$ in $\mathbf{P}^{m m^{\prime}-1}$ with the polydisc of radius I $\left(\left|x_{s, l}\right|<\mathrm{I}\right)$.

If $m=\mathrm{I}$ or $m^{\prime}=\mathrm{I}$, the Veronese embedding is an isomorphism with $\mathbf{P}^{m m^{\prime}-1}$. As a consequence

(4.5.I) If $\operatorname{card}\left(S_{1}\right)=2$ or $\operatorname{card}\left(S_{1}^{\prime}\right)=2$, the cusp point with partition $\left\{S_{1}, S_{1}^{\prime}\right\}$ is non singular.

One can define an algebraic structure on $Q_{88 t}$ by using as a chart at a point $\operatorname{PGL}(2) y$ of $Q_{s t}$ the orbit space

$$
\operatorname{PGL}(2) \backslash\left\{y^{\prime} \in \mathrm{M}_{\mathrm{st}} \mid y^{\prime}(i) \neq y^{\prime}(j) \quad \text { if } y(i) \neq y(j)\right\}
$$

and at a cusp point with partition $\left\{\mathrm{S}_{1}, \mathrm{~S}_{1}^{\prime}\right\}$ the Zariski-open subset of the Veronesc cone of $\mathbf{P}^{m-1} \times \mathbf{P}^{m^{\prime}-1}$ in $\mathbf{C}^{m m^{\prime}}$ given by

$$
x_{s t}=y(s) b y(t) \neq \mathrm{I}, \quad \text { for all } s \in \mathrm{S}_{1}, t \in \mathrm{S}_{1}^{\prime} \text {. }
$$

Example I. - $\mathrm{S}=\{\mathrm{I}, 2,3,4,5\}, \mu_{s}=\frac{2}{5}$ for all $s \in \mathrm{S}$.

Here there are no cusp points: $Q_{8 s t}=Q_{s t}=\operatorname{PGL}(2) \backslash M_{\mathrm{st}}$. Each pair $s, t$ in $\mathrm{S}$ defines the diagonal line $\mathrm{L}_{s, t}: y(s)==y(t)$ in $\mathrm{M}_{\mathrm{st}}$ and under the projection to $\mathrm{Q}_{\mathrm{st}}$, one gets 10 lines. The map of $\mathrm{M}_{\mathrm{st}}$ to $\left(\mathbf{P}^{1}\right)^{2}$ given by

$$
\left(y_{1}, y_{2}, y_{3}, y_{4}, y_{5}\right) \mapsto\left(\infty, o, \mathrm{I}, c\left(y_{1}, y_{2}, y_{3}, y_{4}\right)^{-1}, c\left(y_{1}, y_{2}, y_{3}, y_{5}\right)^{-1}\right)
$$

(where $c$ denotes cross-ratio) descends to a map $p$ of $Q_{\text {st }}$ which is biregular except at $\mathbf{L}_{02}=\rho^{-1}(0,0), \mathbf{L}_{01}=p^{-1}(1, I), L_{12}=p^{-1}(\infty, \infty)$. Herc, $Q_{\text {st }}$ is a blowup of $\mathbf{P}^{1} \times \mathbf{P}^{1}$. Each of the curves $p^{-1}(0,0), p^{-1}(\mathrm{I}, \mathrm{I}), p^{-1}(\infty, \infty)$ are exceptional, that is, each has selfintersection - I. Inasmuch as the set of lines $\left\{\mathrm{L}_{i j}, i \neq j, i, j \in \mathrm{S}\right\}$ are permuted transitively by permutation of coordinates in $\mathrm{M}_{\mathrm{st}}$, thcir images in $\mathbf{Q}_{\mathrm{st}}$ are also permuted transitively and thus each of the ro lines is exceptional. The ro lines of $Q_{\text {st }}$ consist of $p^{-1}(0,0), p^{-1}(I, I), p^{-1}(\infty, \infty)$, and the $p$-lifts of the seven lines on $\mathbf{P}^{1} \times \mathbf{P}^{1}$ :

$$
x=\left\{\begin{array}{l}
0 \\
1, \\
\infty
\end{array}=\left\{\begin{array}{l}
0 \\
\mathrm{I}, \\
\infty
\end{array} \quad x=y\right.\right.
$$

where $x=c\left(y_{1}, y_{2}, y_{3}, y_{4}\right)^{-1}, y=c\left(y_{1}, y_{2}, y_{3}, y_{4}\right)^{-1}$.

Example 2. - $\mathrm{S}=\{\mathrm{I}, 2,3,4,5\}, \mu=\left(\frac{\mathrm{I}}{3}, \frac{\mathrm{I}}{3}, \frac{\mathrm{I}}{3}, \frac{\mathrm{I}}{3}, \frac{2}{3}\right)$.

Here there are four points in $Q_{\text {ousp }}$ corresponding to the partitions $\left\{S_{i}, S_{i}^{\prime}\right\}$ where $\mathrm{S}_{i}=\{i, 5\}, \quad \mathrm{I} \leq i \leq 4 . \quad Q_{\text {8st }}$ is a non-singular manifold by $(4 \cdot 5 \cdot 3)$. This $Q_{\text {88t }}$ can be obtained from the $Q_{\text {sst }}$ of Example 1 by blowing down the four lines $L_{i 5}$ $(\mathrm{I} \leq i \leq 4)$. It can be obtained from $\mathbf{P}^{1} \times \mathbf{P}^{1}$ by blowing up the point $(\infty, \infty)$ and then blowing down the lines $x=\infty$ and $y=\infty$. The resulting space is $\mathbf{P}_{2}$. 
(4.6) We conclude this section by relating our $Q_{\text {sot }}$ to Mumford's when all the $\mu_{s}$ are rational.

Let $\mathcal{O}(n)$ be the line bundle on $\mathbf{P}$ of degree $n$, and let $\Omega_{\mathrm{p}}^{1}$ be the canonical line bundle on $\mathbf{P}$; i.e. $\Omega_{\mathrm{p}}^{1} \cong \mathcal{O}(-2)$. For any line bundle $\mathscr{L}$ we write $\check{\mathscr{L}}$ for the dual line bundle and $\mathscr{L}^{\otimes n}=(\check{\mathscr{L}})^{\otimes(-n)}$ for $n \leq$ o.

Let $\mathrm{D}$ be the common denominator for $\left\{\mu_{s}, s \in \mathrm{S}\right\}$. On $\mathbf{P}^{S}$ define the line bundle

$$
\mathscr{L}_{\mu}=\sum_{s \in \mathrm{S}} \mathcal{O}\left(2 \mathrm{D} \mu_{s}\right)=\bigotimes_{s \in \mathrm{S}} \operatorname{pr}_{s}^{*}\left(\left(\Omega_{\mathrm{P}}^{1}\right)^{\otimes\left(-\mathrm{D} \mu_{s}\right)}\right) \text {. }
$$

It admits a PGL(2)-action coming from the action on the tangent bundle $\check{\Omega}_{\mathrm{p}}^{1} \cong \mathcal{O}(2)$. The stable and semi-stable points of $\mathbf{P}^{\$}$ defined in (4.I) are the same as those defined in Mumford's "Geometric Invariant Theory" for the action of PGL(2) on the linear space of holomorphic sections of $\mathscr{L}_{\mu} ; Q_{\text {s8t }}$ is the underlying topological space of Mumford's "quotient variety" for $\mathrm{PGL}(2) \backslash \mathbf{P}^{\mathrm{S}}\left[{ }_{15}\right]$.

\section{The complex ball}

(5. I) Let $\mathrm{V}$ be a complex vector space, and (, ) be a Hermitian form on V, of signature $(\mathrm{I}, \operatorname{dim} \mathrm{V}-\mathrm{I})$. The complex ball $\mathrm{B}^{+}$in $\mathbf{P}(\mathrm{V}):=\{\mathrm{I}$-dimensional subspaces of $\mathrm{V}\}$ corresponding to $($,$) is the set of lines in \mathrm{V}$ which arcspanned by a vector $v$ with $(v, v)>0$. The form $($,$) is determined, up to a positive real factor, by \mathbf{B}^{+}$. The closure $\overline{\mathbf{B}}^{+}$of $\mathbf{B}^{+}$ in $\mathbf{P}(\mathrm{V})$ is the set of lines spanned by a non zero vector $v$ such that $(v, v) \geq 0$.

(5.2) The hyperbolic angle $\theta \in \mathbf{R}^{+}$between lines $\ell_{1}, \ell_{2} \in \mathrm{B}^{+}$, spanned by vectors $v_{1}, v_{2}$, is defined by

$$
\left|\left(v_{1}, v_{2}\right)\right|=\cosh (\theta)\left(v_{1}, v_{2}\right)^{1 / 2}\left(v_{2}, v_{2}\right)^{1 / 2} .
$$

It remains unchanged when $($,$) is replaced by a positive multiple.$

The angle $\theta\left(\ell_{1}, \ell_{2}\right)$ is a Riemannian distance on $\mathrm{B}^{+}$. It is invariant by the action of the unitary group $\mathrm{U}(\mathrm{V})$ on $\mathrm{B}^{+}$and renders $\mathrm{B}^{+}$a Hermitian symmetric space.

By continuity we extend the function $\theta\left(\ell_{1},-\right)$ to a function $\theta\left(\ell_{1},-\right)$ from $\overline{\mathbf{B}}^{+}$to $\mathbf{R}^{+} \cup\{\infty\}: \theta\left(\ell_{1}, \ell\right)=\infty$ for $\ell \in \partial \mathbf{B}^{+}$. on $\overline{\mathrm{B}}^{+}$by

(5.3) Fix $\ell \in \partial \mathrm{B}^{\vdash}$. For $v$ non zero in $\ell$, we define a "distance from $\ell$ " function

$$
\begin{array}{ll}
d_{v}\left(\ell_{1}\right)=\left|\left(v, v_{1}\right)\right| /\left(v_{1}, v_{1}\right)^{1 / 2} & \text { for } \ell_{1} \in \mathrm{B}^{+}, \text {any } v_{1} \neq 0 \text { in } \ell_{1} \\
d_{v}(\ell)=0, \quad d_{v}\left(\ell^{\prime}\right)=\infty & \text { for } \ell^{\prime} \in \partial \mathrm{B}^{+}, \ell^{\prime} \neq \ell .
\end{array}
$$

The function $\mathrm{d}_{v}$ is continuous and $>0$ on $\overline{\mathrm{B}}^{+}-\{\ell\}$. One has $d_{\lambda v}\left(\ell_{1}\right)=|\lambda| d_{v}\left(\ell_{1}\right)$, and the family of functions $d_{v}(v \in \ell-\{o\})$ is stable by the stabilizer of $\ell$ in $\mathrm{U}(\mathrm{V})$.

(5.4) On $\overline{\mathrm{B}}^{+}$, we will use the coarsest topology for which the functions $d_{v}$ and $\theta\left(\ell_{1},\right)$ are continuous. A fundamental system of neighborhoods of $\ell \in \mathrm{B}^{+}$(resp. of $\ell \in \partial \mathrm{B}^{+}-$on 
which we choose $v \neq 0)$ consists of the $V_{\varepsilon}(\varepsilon>0)$ defined by $V_{\varepsilon}=\left\{\left(\ell_{1} \in B^{+} \mid \theta\left(\ell, \ell_{1}\right)<\varepsilon\right\}\right.$ (resp. $\mathrm{V}_{z}=\left\{\ell_{1} \mid \ell_{1}=\ell\right.$ or $\ell_{1} \in \mathrm{B}^{+}$and $\left.d_{v}\left(\ell_{1}\right)<\varepsilon\right\}$ ).

This topology is finer than the one induced by the topology of $\mathbf{P}(\mathrm{V})$. It induces the usual topology on $\mathrm{B}^{+}$, and the discrete one on $\partial \mathrm{B}^{+}$. It is respected by $\mathrm{U}(\mathrm{V})$.

Proposition (5.5). - Fix $\ell \in \partial \mathrm{B}^{+}$and let $\Delta$ be a discrete subgroup of the projectioe unitary group $\mathrm{PU}(\mathrm{V})$ stabilizing $\ell$ and respecting $a$ " distance to $\ell$ " function $d_{\mathrm{v}}$. If $\ell$ has a neighborhood $\mathrm{V}$ (in the topology 5.4) such that $\mathrm{V} / \Delta$ is compact, the volume of $\left(\mathrm{V} \cap \mathrm{B}^{+}\right) / \Delta$ is finite.

The subgroup of $\mathrm{PU}(\mathrm{V})$ respecting $\ell$ and $d_{v}$ is the isomorphic image of the stabilizer $\mathrm{H}$ of $v$ in $\mathrm{U}(\mathrm{V})$. It is an extension of the unitary group $\mathrm{U}\left(v^{1} / v\right)$ by the unipotent subgroup $\mathrm{N}$ of $\mathrm{H}$ consisting of the $n \in \mathrm{U}(\mathrm{V})$ respecting the filtration $\mathrm{V} \supset v^{\perp} \supset \mathrm{C} v \supset$ o and acting trivially on the successive quotients. The extension is split: $\mathrm{H}$ and $\mathrm{N}$ act transitively on each horocycle $d_{v}\left(\ell_{1}\right)=\mathrm{G}$ and the stabilizer of $\ell_{1}$ is a lifting of $\mathrm{U}^{\top}\left(v^{\perp} / v\right)$.

Fix an isotropic vector $v^{\prime}$ such that $\left(v, v^{\prime}\right)=\mathrm{I}$, and let $v_{1}=v+2 v^{\prime}$. The line $\ell_{1}$ spanned by $v_{1}$ is in $\mathrm{B}^{+}$on the horocycle $d_{v}=1$. Let $\mathrm{V}^{\prime}$ be spanned by $v$ and $v^{\prime}$, and let $V^{\prime \prime}$ be the orthogonal complement $\left(\approx v^{\perp} / v\right)$. Any $h \in \mathrm{H}$ fixing $\ell_{1}$ fixes each point of $V^{\prime}$; hence the stabilizer of $\ell_{1}$ in $H$ is $U\left(V^{\prime \prime}\right) \subset U(V)$. Let $A \subset U\left(V^{\prime}\right) \subset U(V)$ be the group of unitary transformations $a(\lambda): v \mapsto \lambda^{-1} v, v^{\prime} \mapsto \lambda v^{\prime}\left(\lambda \in \mathbf{R}^{+}\right)$and fixing each point of $\mathrm{V}^{\prime \prime}$. It normalizes $\mathrm{H}$ : one has $d_{v} \circ a(\lambda)=d_{a(\lambda)^{-\mathrm{o}_{v}}}=\lambda d_{v^{*}}$ Let $\mathrm{A}^{\mathrm{R}}=\{a(\lambda) \mid \lambda \leq \mathrm{R}\}$. The map $g \mapsto g \ell_{1}$ maps isomorphically $\mathrm{A}^{\mathrm{R}} \mathrm{H} / \mathrm{U}\left(\mathrm{V}^{\prime \prime}\right)=\mathrm{A}^{\mathrm{R}} \mathrm{N}$ onto $\left\{\ell_{2} \in \mathrm{B}^{+} \mid d_{v}\left(\ell_{2}\right) \leq \mathrm{R}\right\}$. The horocycles are closed in $\bar{B}^{+}$(for the topology 5.4). The compactness assumption implies that for $R$ small enough, $\left\{\ell_{2} \mid d_{v}\left(\ell_{2}\right)=R\right\} / \Delta$ is compact. One has

$$
\Delta \backslash a(\mathrm{R}) \mathrm{H} / \mathrm{U}\left(\mathrm{V}^{\prime \prime}\right) \stackrel{\sim}{\rightarrow}\left\{\ell_{2} \mid d_{v}\left(\ell_{2}\right)=\mathrm{R}\right\} / \Delta
$$

and $\Delta$ is hence cocompact in $H$. It remains to evaluate the volume of

$$
\Delta \backslash \mathrm{A}^{\mathrm{R}} \mathrm{H} / \mathrm{U}\left(\mathrm{V}^{\prime \prime}\right) \stackrel{\sim}{\rightarrow}\left\{\ell_{2} \in \mathrm{B} \mid d_{v}\left(\ell_{2}\right) \leq \mathrm{R}\right\} / \Delta
$$

for a volume element on $A^{R} H / U\left(V^{\prime \prime}\right)$ which is $A H$-invariant, hence a multiple of the image of $d a$.dh on $\mathrm{A}^{\mathrm{R}} \mathrm{H}$. It is

$$
\int_{\Delta \backslash \Lambda^{\mathbb{R}} \mathrm{H}} d a \cdot d h=\int_{\lambda \leq \mathrm{K}} \frac{d \lambda}{\lambda} \int_{\Delta \backslash a^{\prime}(\lambda) \mathrm{H}} d h
$$

and

$$
\begin{aligned}
\int_{\Delta \backslash a(\lambda) \mathrm{H}} d h=\int_{a(\lambda)^{-1} \Delta a(\lambda) \backslash \mathrm{HI}} d h=\int_{\Delta \backslash \mathrm{H}} d\left(a(\lambda)^{-1} h a(\lambda)\right) \\
\quad=\mid \operatorname{det}\left(\operatorname{Int} a(\lambda)^{-1}, \text { Lie H }\right) \mid \int_{\Delta \backslash \mathrm{H}} d h .
\end{aligned}
$$

This leads us to evaluate $\int_{\lambda \leq K} \operatorname{det}\left(\operatorname{Int} a(\lambda)^{-1}\right.$, Lic H). One checks that the character $\operatorname{det}\left(\right.$ Int $a(\lambda)^{-1}$, Lie $\left.\mathbf{H}\right)$ of $\lambda \in \mathbf{R}^{+*}$ tends to zero for $\lambda \rightarrow 0$, and the intcgral is hence finite.

The computation above is of course exactly the one used to prove that Siegel domains have finite volume ([2 $b]$, Lemma I.9). 


\section{Stable points}

(6. I) Notation and assumptions (4.0) are in force in this section as well as the assumption $N \geqslant 4$. Our aim is to investigate the asymptotic behaviour of the section $\omega_{\mu}$ of $\mathrm{B}(\boldsymbol{x})_{\mathrm{M}}^{+}$, for $y \in \mathrm{M}$ converging to a stable point $m \in \mathrm{M}_{\mathrm{st}}$.

Fix $m \in \mathrm{M}_{s t}$. To make a local study of $\omega_{\mu}$ near $m$, it will be convenient to identify $\mathbf{P}$ with $\mathbf{P}^{1}$, in such a way that $\infty \notin m(\mathrm{~S})$. Let $\mathrm{U}$ be an open neighborhood of $m$ in $\mathrm{M}_{\mathrm{st}}$, such that $\infty \notin y(\mathrm{~S})$ for $y \in \mathrm{U}$. On the inverse image $\mathrm{P}_{\mathrm{U}}$ of $\mathrm{U}$ in $\mathrm{P}_{\mathrm{p}^{\mathrm{s}}}$ (3.I), therc is a rank one local system, trivialized at $\infty$ by a section $e$ over $\{\infty\} \times U$, and with monodromy $\alpha$ on $\mathrm{P}_{y}$ for $y \in \mathrm{U} \cap \mathrm{M}$. A model of it is: the local system of constant multiples of $\prod_{s \in \mathrm{S}}(z-y(s))^{\mu_{s}}$; the $\mu_{s}$ summing up to an integer, there is no ramification at $\infty$. A trivialisation for large positive real $z$ is provided by the principal determination of $\prod_{\delta \in \mathrm{S}}(z-y(s))^{\mu_{s}}$; it extends by continuity to a trivialisation $e$ at $\infty$. We normalize the Hermitian form $($,$) on \mathrm{L}$ so that $(e, e)=\mathrm{I}$.

For a general $y \in \mathrm{U}$, the monodromy of $\mathrm{L}_{y}$ on $\mathrm{P}_{y}$ around $p \in y(\mathrm{~S})$ is $\prod_{y(s)=p} \alpha_{s}=\exp \left(2 \pi i \sum_{y(s)=p} \mu_{s}\right)$. The stability of $y$ ensures that $o<\sum_{y(s)=p} \mu_{s}<$ I, so that this local monodromy is never trivial.

Fiber by fiber, we take as section $\omega$ of $\Omega^{1}\left(\Sigma \mu_{s} . s\right)(\mathrm{L})$

$$
\omega_{y}=\prod_{s \in \mathrm{S}}(z-y(s))^{-\mu_{s}} . e . d z \text {. }
$$

On $\mathrm{U} \cap \mathrm{M}$, it depends holomorphically on $y$. The assumption of stability of $y$ amounts to saying that this form is of the first kind.

(6.2) The sheaf $R^{1} \pi ! L_{U}$, for $\pi$ the projection $P_{U} \rightarrow U$, is no longer a local system if $m \notin \mathrm{M}$. Its fiber at $m$ is $\mathrm{H}_{c}^{1}\left(\mathrm{P}_{m}, \mathrm{~L}_{m}\right)\left(\stackrel{\sim}{\rightarrow} \mathrm{H}^{1}\left(\mathrm{P}_{m}, \mathrm{~L}_{m}\right)\right.$ by $\left.(2.6 .1)\right)$. For each $h \in \mathrm{H}_{c}^{1}\left(\mathrm{P}_{m}, \mathrm{~L}_{m}\right)$, there is hence a unique germ $h^{b}$ of a section of $\mathrm{R}^{1} \pi_{1} \mathrm{~L}_{\mathrm{U}}$ inducing $h$ on $\mathrm{P}_{m}$. The vector space $\mathrm{H}_{c}^{1}\left(\mathrm{P}_{m}, \mathrm{~L}_{m}\right)$ being finite dimensional, this provides us on $\mathrm{U}$ suitably small, with a map

$$
\text { (constant sheaf } \left.\mathrm{H}_{c}^{1}\left(\mathrm{P}_{m}, \mathrm{~L}_{m}\right)\right) \rightarrow \mathrm{R}^{1} \pi_{!} \mathrm{L}_{\mathrm{U}} \text {. }
$$

This map is compatible with the non degenerate Hermitian form ( , ), and hence injective. Compatibility can be checked on the description given below. In particular, each $h \in \mathrm{H}_{c}^{1}\left(\mathrm{P}_{m}, \mathrm{~L}_{m}\right)$ defines a horizontal section $h^{b}$ of $\mathrm{R}^{1} \pi_{1} \mathrm{I}_{4}$ : on $\mathrm{U} \cap \mathrm{M}$. Wc describe $h^{b}$, for $h$ the class of a $\mathrm{L}_{m}$-valued compactly supported $\mathrm{C}^{\infty}$ form $\eta$, whose support is contained in a connected compact $\mathrm{K}$ containing $\infty$, and for $\mathrm{U}$ connected and small enough for $\mathrm{K}$ to be disjoint from $y(\mathrm{~S})$ for $y \in \mathrm{U}$. On $\mathrm{K} \times \mathrm{U} \subset \mathrm{P}_{\mathrm{U}}$, L is uniquely isomorphic to $\mathrm{pr}_{1}^{*}\left(\mathrm{~L}_{m}\right)$, by an isomorphism which is the identity above $m$. It hencc make sense, in each fibre $\mathrm{P}_{y}$, to take the same form $\eta$; it defines $h_{y}^{b}$. 
(6.3) If we apply this to $\omega_{m}$, we get an horizontal section $\operatorname{cl}\left(\omega_{m}\right)^{b}$ of $R^{1} \pi_{1} L_{U}$ on $\mathrm{U} \cap \mathrm{M}$. As $\left(\omega_{m}, \omega_{m}\right)>0(2.20)$, the same positivity holds for $\operatorname{cl}\left(\omega_{m}\right)^{b}$, and, projecting from $R^{1} \pi_{1} L_{U}$ to $B(\alpha)_{M}$, we get a section $\operatorname{Pcl}\left(\omega_{m}\right)^{b}$ of $B(\alpha)_{M}^{+}$. We will compare it with the holomorphic section $w_{\mu}$, using fiber by fiber the metric $(5 \cdot 2)$.

Proposition (6.4). - $\theta\left(w_{\mu}, \operatorname{Pcl}\left(\omega_{m}\right)^{b}\right)$ tends to o for $y \in \mathrm{M}$ tending to $\mathrm{m}$.

Proof. - This will result at once from the two statements

(6.4.1 $\quad\left(\omega_{y}, \omega_{y}\right) \rightarrow\left(\omega_{m}, \omega_{m}\right) \quad$ for $y \rightarrow m$;

(6.4.2) $\quad\left(\omega_{y}, \operatorname{cl}\left(\omega_{m}\right)^{b}\right) \rightarrow\left(\omega_{m}, \omega_{m}\right)$ for $y \rightarrow m$.

If it made sense, we would like to say that $\omega_{y} \rightarrow \omega_{m}$ in the $\mathrm{L}^{2}$-norm, for $y \rightarrow m$, but $\omega_{y}$ and $\omega_{m}$ do not live in the same space, having different ramification. Let $K_{R}$ be the complement in $\mathbf{P}^{1}$ of discs of radius $\mathbf{R}$ around each point of $m(\mathbf{S})$. Onc assumes $R$ small enough for these discs to be disjoint; on a connected neighborhood $\mathrm{U}$ of $y$, small enough for $y(S)$ to stay disjoint from $K_{R}$, one can compare $\omega_{y} \mid K_{R}$ and $\omega_{m} \mid K_{R}$ : on $\mathrm{K}_{\mathrm{R}} \times \mathrm{U}, \mathrm{L} \sim \mathrm{pr}_{1}^{*} \mathrm{~L}_{m}$. There, one has a $\mathrm{C}^{\infty}$-convergence of $\omega_{y}$ to $\omega_{m}$. To prove (6.4. I), it hence suffices to obtain a bound $\varepsilon(R)$, uniform in $y$, close to $m$, for the $\mathrm{L}^{2}$-norm of the restriction of $\omega_{y}$ to a disk $D_{R}$ of radius $R$ around $p \in m(s)$, for any $s \in S$, this bound being such that $\varepsilon(R) \rightarrow 0$ for $R \rightarrow 0$.

We may assume $\mathrm{R}$ and $\mathrm{U}$ small enough so that $y(s) \notin \mathrm{D}_{2 \mathrm{R}}$ if $m(s) \neq p$. This ensures

$$
\Pi|z-y(s)|^{-\mu_{s}}<\text { constant. } \prod_{m(s)=p}|z-y(s)|^{-\mu_{s}},
$$

and the required estimate follows from the following lemma.

Lemma (6.5). - Let $\mathrm{D}$ be a disc of radius $\mathrm{R},\left(a_{i}\right)_{i \in \mathrm{I}}$ be a family of points of $\mathrm{D}$ and $\left(\mu_{i}\right)_{i \in \mathrm{I}}$ a family of real numbers, such that $0<\mu_{i}<_{\mathrm{I}}$ and $\Sigma \mu_{i}<\mathrm{I}$. Then, writing $z=x+i y$, one has

$$
\int_{\mathrm{D}} \Pi\left|z-a_{i}\right|^{-2 \mu_{i}} d x d y \leq \int_{|z|<\mathrm{R}}|z|^{-2 \Sigma \mu_{i}} d x d y=\frac{2 \pi \mathrm{R}^{2\left(1-\Sigma \mu_{i}\right)}}{2\left(\mathrm{I}-\Sigma \mu_{i}\right)}
$$

Proof. - We may and shall assume D centered at o. If the real numbers $\boldsymbol{p}_{i}$ are such that $I>\frac{1}{p_{i}}>\mu_{i}$ and $\Sigma \frac{I}{p_{i}}=I$, Holder's inequality gives

$$
\int_{\mathrm{D}} \Pi\left|z-a_{i}\right|^{-2 \mu_{i}} d x d y \leq \Pi\left[\int_{\mathrm{D}}\left|z-a_{i}\right|^{-2 \mu_{i} p_{i}} d x d y\right]^{1 / p_{i}}
$$

One has

$$
\int_{\mathrm{D}}\left|z-a_{i}\right|^{-2 \mu_{i} p_{i}} d x d y \leq \int_{\mathrm{D}}|z|^{-2 \mu_{i} p_{i}} d x d y=2 \pi \int_{0}^{\mathrm{R}} r^{1-2 \mu_{i} p_{i}} d x=2 \pi \frac{\mathrm{R}^{2-2 \mu_{i} p_{i}}}{2-2 \mu_{i} p_{i}}
$$


that the integral of $\left|z-a_{i}\right|^{-2 \mu_{i}} p_{i} d x d y$ is bigger when taken on the disc $\mathrm{D}_{\mathrm{R}}\left(a_{i}\right)$ of radius $R$ and center $a_{i}$ than when taken on $D_{R}(0)$ results from the fact that $\left|z-a_{i}\right|^{-2 i_{i} p_{i}}$ is bigger on $\mathrm{D}_{\mathrm{R}}\left(a_{i}\right)-\mathrm{D}_{\mathrm{R}}(\mathrm{o})$ than on $\mathrm{D}_{\mathrm{R}}(\mathrm{o})-\mathrm{D}_{\mathrm{R}}\left(a_{i}\right)$ : one has $\left|z-a_{i}\right| \leq \mathrm{R}$ on $\mathrm{D}_{\mathrm{R}}\left(a_{\mathrm{i}}\right)-\mathrm{D}_{\mathrm{R}}(\mathrm{o})$, and $\left|z-a_{i}\right| \geq \mathrm{R}$ on $\mathrm{D}_{\mathrm{R}}(\mathrm{o})-\mathrm{D}_{\mathrm{R}}\left(a_{\mathrm{i}}\right)$.

Using that $\Sigma_{\mathrm{I}} / p_{i}=\mathrm{I}$, we have

$$
\int_{\mathrm{D}} \Pi\left|z-a_{i}\right|^{-2 \mu_{i}} d x d y \leq \Pi\left[2 \pi \cdot \frac{\mathrm{R}^{2-2 i_{i} p_{i}}}{2-2 \mu_{i} p_{i}}\right]^{1 / p_{i}}=2 \pi \cdot \mathrm{R}^{2\left(1-\Sigma_{\mu_{i}}\right)} \cdot \mathrm{C}^{-1}
$$

with $\mathrm{G}=2 . \Pi\left(\mathrm{I}-\mu_{i} p_{i}\right)^{1 / p_{i}}$. Taking the $p_{i}$ such that the $\mu_{i} p_{i}$ are all equal, i.e. $p_{i}=\mu_{i}^{-1} \Sigma \mu_{i}$, one gets the result.

(6.6) Proof of (6.4.2). - For each $p \in m(\mathrm{~S})$, let $\mathrm{D}_{p}$ be a disc of radius $\mathrm{R}$ around $p$, $u_{p}$ the solution on $\mathrm{D}_{p}^{*}=\mathrm{D}_{p}-p$ of $\omega_{m}=d u$ and $\varphi_{p}$ the characteristic function of $\mathrm{D}_{p}$. The current with compact support $\omega_{m}-d \Sigma \varphi_{p} u_{p}$ can be used to compute $\operatorname{cl}\left(\omega_{m}\right)^{b}$. One gets

$$
\left(\omega_{y}, \operatorname{cl}\left(\omega_{m}\right)^{b}\right)=\frac{1}{2 \pi i} \int_{K_{\mathrm{B}}} \omega_{y} \wedge \bar{\omega}_{m}-\sum_{p} \frac{\mathrm{I}}{2 \pi i} \int_{2 \mathrm{D}_{p}}\left\langle\omega_{y}, u_{p}\right\rangle
$$

For $y \rightarrow m$, and $\mathrm{R} \rightarrow \mathrm{o}$ in such a way that the disc of radius $2 \mathrm{R}$ around $p$ contains all $y(s)$ for $m(s)=p$, the first integral converges to $\left(\omega_{m}, \omega_{m}\right)$, while the others cach are $\mathrm{O}\left(\mathrm{R}^{2 \mathrm{~B}}\right), \mathrm{B}=\mathrm{I}-\sum_{m(s)=p} \mu_{s}$. This concludes the proof.

(6.7) In the neighborhood of a stable point $y \in Q_{8 t}$, the projection of $M_{s t}$ to $\mathcal{Q}_{\text {st }}$ has a section. Taking a pull-back by such a section, we get the following:

Corollary (6.8). - For $y \in Q_{\mathrm{st}}$, there exists a neighborhood $\mathrm{V}$ of $y$ in $\mathrm{Q}_{\mathrm{st}}$ and a horizontal section $b_{y}$ of $\mathrm{B}(\alpha)$ on $\mathrm{Q} \cap \mathrm{V}$ such that $\theta\left(b_{y}\left(y^{\prime}\right), w_{\mu}\left(y^{\prime}\right)\right) \rightarrow$ o for $y^{\prime} \rightarrow y \quad\left(y^{\prime} \in Q\right)$.

For $y \in Q$, the corollary just tells once more that the section $w$ is continuous at $y$.

(6.9) A partition $\mathrm{T}$ of $\mathrm{S}$ is stable if for each $\mathrm{G} \in \mathrm{T}, \mu(\mathrm{T})_{\mathrm{C}}:=\sum_{s \in \mathrm{C}} \mu_{s}$ is $<\mathrm{I}$. Define $\alpha(\mathrm{T})_{\mathrm{C}}=\exp \left(2 \pi i \mu(\mathrm{T})_{\mathrm{C}}\right)=\prod_{\mathrm{s} \in \mathrm{C}} \alpha_{\mathrm{s}}$. For $\mathrm{T}$ stable, let $\mathrm{M}_{\mathrm{T}} \subset \mathrm{M}_{\mathrm{st}}$ bc the space of maps $y$ from $\mathrm{S}$ to $\mathrm{P}$, such that $y(s)=y(t)$ if and only if $s$ and $t$ arc in the same coset of $T$. It is the inverse image in $M_{s t}$ of a subspace $Q_{T}$ of $Q_{s t}$. The $M_{T}$ (resp. $Q_{T}$ ) for $T$ stable form a partition of $M_{s t}\left(\right.$ resp. $\left.Q_{s t}\right)$ and each $M_{T}$ (resp. $Q_{\mathrm{T}}$ ) can be identified with the analogue $M(T)$ of $M$ (resp. $Q(T)$ of $Q$ ) for $T, \mu(T)$, and its closure with the analogue $M_{s t}(T)$ (resp. $Q_{s t}(T)$ ) of $M_{s t}$ (resp. $Q_{s t}$ ).

Fix $m, \mathrm{U}, \mathrm{L}$ as in (6.I), with $m \in \mathrm{M}_{\mathrm{T}}$. On $\mathrm{M}_{\mathrm{T}} \cap \mathrm{U}$, the $\mathrm{H}_{c}^{1}\left(\mathrm{P}_{y}, \mathrm{~L}_{y}\right)$ form a local system. By (6.2.I), this local system, which is trivial as $U$ is small enough, extends as a trivial sublocal system of $\mathrm{R}^{1} \pi_{!} \mathrm{L}_{\mathrm{U}}$. The $\omega_{y}\left(y \in \mathrm{M}_{\mathrm{T}} \cap \mathrm{U}\right)$ define a non-zero holomorphic section of this local system on $\mathrm{M}_{\mathrm{T}} \cap \mathrm{U}$. The corresponding ray can be identified with the section $w_{\mu(T)}$ of $\mathbf{B}(\alpha(T))$ on $\mathrm{M}(\mathrm{T})$. 
On $\mathbf{Q}_{\mathrm{st}}$, this gives us in a neighborhood $\mathrm{V}$ of $y \in \mathrm{Q}_{\mathrm{T}}$ :

a) A horizontal sub-ball-bundle of $B(\alpha)$, which extends as a constant horizontal bundle on $V$. Its restriction to $Q_{T}$ can be identified with $B(\alpha(T))$ on $Q(T)$.

b) Via this identification, the horizontal section $b_{y}$ of $(6.8)$ is the horizontal section through $w_{\mu(T)}(y)$.

Proposition (6.10). - If $\mu$ satisfies condition INT of (3.1I): $0<\mu_{8}<1, \quad \Sigma \mu_{\mathrm{s}}=2$ and for all $s \neq t$ in $\mathrm{S}$ such that $\mu_{\mathrm{s}}+\mu_{t}<\mathrm{I}, \quad\left(\mathrm{I}-\mu_{s}-\mu_{t}\right)^{-1}$ is an integer, then any subset $\mathrm{C} \subset \mathrm{S}$ for which $\sum_{s \in \mathrm{C}} \mu_{s}<\mathrm{I}$ has at most three elements, and for any such subset with at least two elements, $\left(1-\sum_{s \in \mathrm{C}} \mu_{s}\right)^{-1}$ is an integer.

The condition INT implies that $\mu_{\mathrm{g}}+\mu_{t}$ is always $\geq I / 2$ for $s \neq \mathrm{t}$. Averaging over the pairs in $\mathrm{C} C \mathrm{~S}$, we get that for $\operatorname{card}(\mathrm{C}) \geq 2$, the moan of the $\mu_{\mathrm{s}}(s \in \mathbf{C})$ is $\geq 1 / 4$. From $\sum_{s \in C} \mu_{s}<_{1}$ there results card $(\mathrm{C})<4$.

The case card $(\mathrm{G})=2$ is trivial by INT. Suppose $\mathbf{G}=\{a, b, c\}$. The relations $\quad \mathrm{I}-\mu_{a}-\mu_{b}=\frac{\mathrm{I}}{n_{a b}}, \quad \mathrm{I}-\mu_{b}-\mu_{c}=\frac{\mathrm{I}}{n_{b c}}, \quad \mathrm{I}-\mu_{c}-\mu_{a}=\frac{\mathrm{I}}{n_{a c}} \quad$ sum up to $\mathrm{I} \cdot \mu_{a}-\mu_{b}-\mu_{c}=\frac{\mathrm{I}}{2}\left(\frac{\mathrm{I}}{n_{a b}}+\frac{\mathrm{I}}{n_{b c}}+\frac{\mathrm{I}}{n_{c a}}-\mathrm{I}\right)$, hence $\frac{\mathrm{I}}{n_{a b}}+\frac{\mathrm{I}}{n_{b c}}+\frac{\mathrm{I}}{n_{c a}}>\mathrm{I}$.

We now use the elementary fact that a triple of integers whose reciprocals have their sum strictly greater than I must be one of

$$
(2,2, n)(2,3,3)(2,3,4)(2,3,5) \text {. }
$$

The excesses of the sums over $\mathrm{I}$ are respectively

$$
\mathrm{I} / n, \mathrm{I} / 6, \mathrm{I} / \mathrm{1} 2, \mathrm{I} / 3 \mathrm{0} \text {. }
$$

It follows at once that $\left(I-\mu_{a}-\mu_{b}-\mu_{c}\right)^{-1}$ is an integer.

Corollary (6.1 $)$. - If $\mu$ satisfies INT and $\mathrm{T}$ is a stable partition of $\mathrm{S}$ (cf. (6.9)), the family $\mu(\mathrm{T})$ defined by

$$
\mu(\mathrm{T})_{\mathrm{C}}=\sum_{s \in \mathrm{C}} \mu_{s} \quad(\mathrm{C} \in \mathrm{T})
$$

again satisfies INT.

\section{Semi-stable points}

(7.r) The notation and assumptions (4.o) are in force in this section; we assume also $N \geqslant 4$. Let $(S(I), S(2))$ be a partition of $S$, such that $\sum_{s \in S(i)} \mu_{s}==I$. We want to investigate the asymptotic behavior on $Q$ of $\omega_{\mu}$, for $y$ converging to a point in $Q_{\text {oupp }}$ of type $(S(1), S(2))$. 
Fix $a \in \mathrm{S}(\mathrm{I})$ and $b \in \mathrm{S}(2)$, identify $\mathbf{P}$ with $\mathbf{P}=\mathbf{P}^{1}$, and let $\mathrm{W}$ be the space of $y \in \mathrm{P}^{\mathbb{8}}$ such that $y(a)=0, y(b)=\infty$, and

$$
\sup \{|y(s)| \mid s \in \mathrm{S}(\mathbf{I})\}<\inf \{|y(s)| \mid s \in \mathrm{S}(\mathbf{2})\}
$$

(as in section 4 where we wrote $\mathrm{V}$ instead of $\mathrm{W}$ ). We put $\mathrm{A}(y)=\sup \{|y(s)| \mid s \in \mathrm{S}(\mathrm{I})\}$, $\mathrm{B}(y)=\inf \{|y(s)| \mid s \in \mathrm{S}(2)\}, \quad \mathrm{C}(y)=\mathrm{A}(y) / \mathrm{B}(y)$. The quotient map $\pi: \mathrm{W} \rightarrow \mathrm{Q}_{8 s t}$ sends a point $y$ with $\mathrm{G}(y)=0$ to the semistable point $\mathrm{J}$ of type (S(I), $\mathrm{S}(2))$ and identifies $\left(\mathrm{W}-\pi^{-1}(\mathrm{~J})\right) / \mathbf{G}_{m}$ with a punctured neighborhood of $\mathrm{J}$. One has convergence of $\pi(y)$ to $\mathrm{J}$ if and only if $\mathrm{C}(y)$ converges to $\mathrm{o}$.

On $\mathrm{P}_{\mathrm{w}}$, there is, up to a unique isomorphism, a unique rank one local system $\mathrm{L}$, trivialized on the annulus $\mathrm{A}(y)<|z|<\mathrm{B}(y)$ by a section $e$, and which for $y \in \mathbf{M}$, has fiberwise monodromy $\propto$ (hence trivial monodromy around the annulus, as $\left.\prod_{s \in \mathbb{B}(i)} \alpha_{s}=1\right)$. A model of it is the local system of constant multiples of

$$
\prod_{s \in \mathbb{B}(1)}(z-y(s))^{\mu_{s}} \cdot \prod_{s \in \mathbb{B}(2)}\left(\mathrm{I}-\frac{z}{y(s)}\right)^{\mu_{s}} \text {. }
$$

We normalize the Hermitian form $($,$) on \mathrm{L}$ by $(e, e)=\mathrm{I}$.

For any $y \in \mathrm{W}$, fix $\mathrm{R}$ such that $\mathrm{A}(y)<\mathrm{R}<\mathrm{B}(y)$, and let $\omega_{\mathrm{J}}$ be the $\mathrm{L}$-valued homology class represented by the cycle $|z|=\mathrm{R}$ in $\mathrm{P}_{y}$, positively oriented and provided with $e$, the trivializing section of $\mathrm{L}$. It can also be viewed as a class in $\mathrm{H}_{c}^{1}\left(\mathbf{P}_{y}, \mathrm{~L}_{y}\right)$. For $y=j$, defined by $j(\mathrm{~S}(\mathrm{I}))=0, j(\mathrm{~S}(2))=\infty$, it is a gencrator of this $\mathrm{H}_{c}^{1}$. The $\omega_{\mathrm{J}}$ provide a horizontal section of $R^{1} \pi_{l} L$. Since $\left(\omega_{\mathrm{J}}, \omega_{\mathrm{J}}\right)=0$, the line $\left(\omega_{\mathrm{J}}\right)$ spanned by $\omega_{\mathrm{J}}$ is in the boundary of the ball $\mathrm{B}^{+}(\alpha)$, and the functions $d_{\omega_{\mathrm{J}}}$ of $(5 \cdot 3)$ provide a horizontal family of " distance to $\mathrm{P} \omega_{\mathrm{J}}$ " functions. We will use them to compare $\mathrm{P} \omega_{\mathrm{J}}$ with the holomorphic section $w_{\mu}$ of $\mathrm{B}(\alpha)^{+*}$.

Proposition (7.2). - For y variable in $\mathrm{W}$, if $\mathrm{G}(y)$ tends to 0 , the $d_{\omega_{J}}\left(w_{\mu}\right)$ tend to o.

Proof. - Fiber by fiber $w_{\mu}$ is the line spanned by the cohomology class of

$$
\omega_{y}=\prod_{s \in \mathbb{S}(1)}(z-y(s))^{-\mu_{s}} . \prod_{s \in \mathbb{B}(2)}\left(1-\frac{z}{y(s)}\right)^{-\mu_{s}} . d z . e .
$$

The form $\omega_{y}$, as a function of $y$, is invariant by the action of $\mathbf{G}_{m}$ on $(\mathrm{W}, \mathrm{L})$. It hence suffices to treat the case where $\mathrm{A}(y) \rightarrow 0, \mathrm{~B}(y) \rightarrow \infty$. In that case, the numerator of

$$
d_{\omega_{\mathrm{J}}}\left(w_{\mu}\right)=\left|\left(\omega_{\mathrm{J}}, \omega_{y}\right)\right| /\left(\omega_{y}, \omega_{y}\right)^{1 / 2}
$$

clearly tends to $\mathrm{r}$ : it is the absolute value of

$$
\frac{\mathrm{I}}{2 \pi i} \oint_{|z|=1} \prod_{s \in \mathrm{s}(1)}(z-y(s))^{-\mu_{s}} \cdot \prod_{s \in \mathrm{B}(2)}\left(\mathrm{I}-\frac{z}{y(s)}\right)^{-\mu_{s}} . d z \rightarrow \frac{\mathrm{I}}{2 \pi i} \oint \frac{d z}{z}=1 \text {. }
$$


We have to prove that $\left(\omega_{y}, \omega_{y}\right) \rightarrow \infty$. Indeed,

$$
\begin{aligned}
\left(\omega_{y}, \omega_{y}\right) \geq \frac{\mathrm{I}}{2 \pi} \int_{2 \mathrm{~A}(y)<|z|<\frac{1}{2} \mathrm{~B}(y)} \prod_{\mathbf{S}(1)}|z-y(s)|^{-2 \mu_{s}} \cdot \prod_{\mathbf{8}(2)}\left|\mathrm{I}-\frac{z}{y(s)}\right|^{-2 \mu_{s}} \cdot|d z \wedge d \bar{z}| \\
\sim \int_{2 \mathrm{~A}(y)}^{\frac{1}{2} \mathrm{~B}(y)} 2 \cdot \frac{d r}{r} \sim 2|\log \mathrm{C}(y)| .
\end{aligned}
$$

On Q, the proposition (7.2) has the following corollary:

Corollary (7.3). - For each semi-stable point y of $\mathbf{Q}_{\text {ousp }}$, there is on a neighborhood $\mathrm{V}$ of $y$ a horizontal family of functions $d$ on the balls $\mathrm{B}(\alpha)_{y^{\prime}}\left(y^{\prime} \in \mathrm{V} \cap \mathrm{Q}\right)$-each a " distance to a point of $\mathrm{\partial B}(\alpha)_{y^{\prime}}$ " function-such that $d\left(w_{\mu}\left(y^{\prime}\right)\right) \rightarrow 0$ for $y^{\prime} \rightarrow y$.

\section{Extending $\widetilde{w}_{\mu}$ by continuity}

Let $\mathrm{X}, \mathrm{Y}$ be complex algebraic varieties which are separated as algebraic varieties, i.e. which are Hausdorff in their complex topology.

(8. I) One knows that any quasi-finite map $f: \mathrm{X} \rightarrow \mathrm{Y}$-i.e. a map whose fibers are finite-admits a factorization $\mathrm{X} \stackrel{j}{\rightarrow} \overline{\mathrm{X}} \stackrel{i}{\rightarrow} \mathrm{Y}$, with $j$ an open embedding, and $\bar{f}$ a finite map (= quasi-finite and proper). If $\mathrm{X}$ and $\mathrm{Y}$ are connected and normal, of the same dimension, there is an unique such factorization for which $\overline{\mathrm{X}}$ is normal. One calls this $\overline{\mathrm{X}}$ the normalization of $\mathrm{Y}$ in $\mathrm{X}$.

For more general spaces, and for some maps with totally disconnected but possibly infinite fibers, a simple topological generalization of this construction has becn given by R. H. Fox [7].

The spaces $\mathrm{X}$ and $\mathrm{Y}$ are taken to be Hausdorff and locally connected. A continuous map $f: \mathrm{X} \rightarrow \mathrm{Y}$ is called a spread if, for any $x$ in $\mathrm{X}$, one obtains a fundamental system of neighborhoods of $x$ by taking, for each neighborhood $\mathrm{V}$ of $f(x)$, the connected component of $f^{-1}(\mathrm{~V})$ containing $x$. A spread $f$ is called complete if, for any $y \in \mathrm{Y}$,

$$
f^{-1}(y) \stackrel{\sim}{\rightarrow} \operatorname{proj} \lim \pi_{0}\left(f^{-1}(\mathrm{~V})\right),
$$

the projective limit being taken on the neighborhoods $\mathrm{V}$ of $y$.

Fox proves that cach spread $f: \mathrm{X} \rightarrow \mathrm{Y}$ can bc embedded in a complete spread $\bar{f}: \overline{\mathrm{X}} \rightarrow \mathrm{Y}$ with the following universal property: any commutative diagram of solid arrows

(8.1.1)

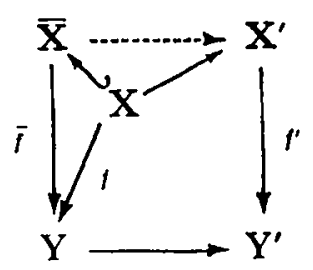


with $f^{\prime}$ a complete spread, can be completed as shown. For any $y \in \mathrm{Y}$, one has

$$
\bar{f}^{-1}(y) \stackrel{\sim}{\rightarrow} \text { proj } \lim \pi_{0}\left(f^{-1}(\mathrm{~V})\right),
$$

the projective limit being taken on the neighborhoods $\mathrm{V}$ of $y$. It is clear from the universal property that $\overline{\mathrm{X}}$ is unique up to unique isomorphism, and functorial. We will call it the completion of $\mathrm{X}$ over $\mathrm{Y}$. It contains $\mathrm{X}$ as a dense subspace, and the topology of $X$ is induced by that of $\bar{X}$. The space $\bar{X}$ is locally connected.

(8.2) We define a space $\mathrm{X}$ to be connected if it is non-empty and has no proper open and closed subset.

A map $f: \mathrm{X} \rightarrow \mathrm{Y}$ is called a covering map if each $y \in \mathrm{Y}$ has a neighborhood $\mathrm{V}$ for which there exists a discrete set $\mathbf{D}$ (possibly cmpty), and a commutative diagram

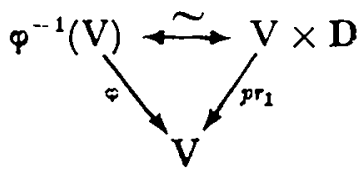

If $\mathrm{Y}$ is connected and $\mathrm{X}$ non-empty, such a map is onto.

A map $f: X \rightarrow Y$ is called a local homeomorphism at $x \in \mathrm{X}$ if $x$ has a neighborhood $\mathrm{U}$ which maps homeomorphically onto a neighborhood of $f(x)$. The map $f$ is called a local homeomorphism if this holds at all points $x \in \mathrm{X}$.

Assume that $\mathrm{X}$ is a connected covering space of a connected locally simply connected open subset $U$ of $Y$. The composed map $X \rightarrow U \hookrightarrow Y$ is then a spread. Assume further that each $y$ in $\mathrm{Y}$ has a fundamental system $\mathscr{V}_{y}$ of open neighborhoods such that

$$
\text { for } \mathrm{V} \text { in } \mathscr{V}_{y}, \mathrm{~V} \cap \mathrm{U} \text { is connected; }
$$

As $f$ is a local homeomorphism, $\mathrm{X}$ is open in $\overline{\mathrm{X}}$. As $\mathrm{X}$ is a covering of $\mathrm{U}$, one has further $\mathrm{X}=\bar{f}^{-1}(\mathrm{U})$.

Fix a base point $o \in U$. One knows that the functor $(g: \tilde{U} \rightarrow U) \mapsto g^{-1}(o)$ is an equivalence of the category of coverings of $U$ with that of discrete sets on which $\pi_{1}(U, 0)$ acts. In particular, the connectcd components of $\tilde{U}$ correspond to the orbits of $\pi_{1}(\mathrm{U}, o)$ on $g^{-1}(o)$. For $W$ open in $U$, connected and containing $o$, the same applies to $\mathrm{W}$ and the restriction functor $(g: \widetilde{U} \rightarrow U) \mapsto\left(g^{-1}(W) \rightarrow W\right)$ from coverings of $U$ to coverings of $W$ corresponds to the functor: restriction of the action of $\pi_{1}(U, 0)$ to $\pi_{1}(W, 0)$. In particular, if $\tilde{U}$ corresponds to $\pi_{1}(U, 0) / K, \pi_{0}\left(g^{-1} W\right)$ is $\pi_{1}(W, 0) \backslash \pi_{1}(U, 0) / K$.

This applies to X. If a lifting $\tilde{o}$ of $o$ is chosen in $X$, the map $\pi_{1}(U, o) \rightarrow f^{-1}(o): \sigma \mapsto \sigma . \widetilde{o}$ identifies $f^{-1}(o)$ with a suitable homogeneous space $\pi_{1}(\mathrm{U}, o) / \mathrm{K}$ of $\pi_{1}(\mathrm{U}, 0)$.

Fix $y \in \mathrm{Y}$, and a neighborhood $\mathrm{V} \in \mathscr{V}_{y}$. Let us assume at first that $o \in \mathrm{V}$. The decomposition group $\mathrm{D}_{\nu}$ is then defined as the image of $\pi_{1}(\mathrm{~V} \cap \mathrm{U}, 0)$ in $\pi_{1}(\mathrm{U}, 0)$ and one has

$$
\pi_{0}\left(f^{-1}(\mathrm{~V} \cap \mathrm{U})\right)=\mathrm{D}_{y} \backslash \pi_{1}(\mathrm{U}, o) / \mathrm{K}
$$


For $V^{\prime} \subset V$ in $\mathscr{V}_{y},(8.2 .1)$ and (8.2.2) imply that $\pi_{0}\left(f^{-1}\left(V^{\prime} \cap U\right)\right) \rightarrow \pi_{0}\left(f^{-1}(V \cap U)\right)$ hence

$$
\bar{f}^{-1}(y)=\mathrm{D}_{y} \backslash \pi_{1}(\mathrm{U}, o) / \mathrm{K} .
$$

For $\sigma \in \pi_{1}(\mathrm{U}, o) / \mathrm{K}, \sigma . \tilde{o}$ is a lifting of $o$ in the connected component $f^{-1}(\mathrm{~V})_{\circ}$ of $f^{-1}(\mathrm{~V})$ corresponding to the double coset $\mathrm{D}_{y} \sigma \mathrm{K}$. If we use it as a base point, the fibre at $o$ of the covering $f^{-1}(\mathrm{~V})_{\sigma}$ of $\mathrm{V} \cap \mathrm{U}$ is identified with $\mathrm{D}_{y} \sigma \mathrm{K} / \mathrm{K}=\mathrm{D}_{y} / \mathrm{D}_{y} \cap \sigma \mathrm{K} \sigma^{-1}$. To the component $f^{-1}(\mathrm{~V})_{\sigma}$ there corresponds a point $\bar{y}$ in $\bar{f}^{-1}(y)$. The $f^{-1}(\mathrm{~V})_{\sigma} \cap f^{-1}\left(\mathrm{~V}^{\prime}\right)$, for $\mathrm{V}^{\prime}$ a neighborhood of $y$, are the trace on $\mathrm{X}$ of a fundamental system of neighborhood of $\bar{y}$ in $\overline{\mathrm{X}}$.

For $o$ not necessarily in $\mathrm{V}$, one chooses $o^{\prime}$ in $\mathrm{V} \in \mathrm{U}$ and a path $p$ from $o$ to $o^{\prime}$ in $\mathrm{U}$. This path lifts as a path from $\widetilde{o}$ to some lifting $\widetilde{o}^{\prime}$ et $o^{\prime}$. It defines an isomorphism of $\pi_{1}(\mathrm{U}, 0)$ with $\pi_{1}\left(\mathrm{U}, o^{\prime}\right)$ via which the above constructions, which make sense for $o^{\prime}$, can be pushed back into $\pi_{1}(\mathrm{U}, o)$. For instance, the decomposition group $\mathrm{D}_{y} \subset \pi_{1}(\mathrm{U}, o)$ is defined as the image of the decomposition group $D_{y} \subset \pi_{1}\left(U, o^{\prime}\right)$. It depends on the path $p$. One still has $(8 \cdot 2 \cdot 3)$.

(8.2.4) A covering map $f: \mathrm{X} \rightarrow \mathrm{U}$ is normal if the $\pi_{1}(\mathrm{U}, 0)$ homogeneous space $f^{-1}(0)$ is isomorphic to a homogeneous spaces $\pi_{1}(\mathrm{U}, o) / \mathrm{K}$, with $\mathrm{K}$ a normal subgroup. If $f: \mathrm{X} \rightarrow \mathrm{U}$ is a normal covering map, the group $\pi_{1}(\mathrm{U}, 0)$ acts on $\mathrm{X}$ by deck-transformations, and this action extends to $\overline{\mathrm{X}}$ by functoriality. We leave to the reader to check that, under the conditions $(8.2 .1),(8.2 .2)$, one has

I) $\pi_{1}(\mathrm{U}, o) \backslash \overline{\mathrm{X}} \stackrel{\sim}{\rightarrow} \mathrm{Y}$;

2) for each $y \in \mathrm{Y}$, the stabilizer in $\pi_{1}(\mathrm{U}, o)$ of a point $\tilde{y}$ in $\bar{f}^{-1}(y)$ is the conjugate of the decomposition group $\mathrm{D}_{\boldsymbol{y}}$ determined by a path in $\mathrm{U}$ corresponding to $\tilde{y}$.

Remark. - Let $\mathrm{K}$ denote the kernel of the action of $\pi_{1}(\mathrm{U}, o)$ on $\mathrm{X}$. Then $\pi_{1}(\mathrm{U}, 0) / \mathrm{K}$ acts properly discontinuously on $\mathrm{X}$ but not necessarily on $\overline{\mathrm{X}}$.

(8.3) Let $w$ be a continuous function from $X$ to a topological space $B$. We assume $B$ regular, i.e. that any neighborhood of any point contains a closed neighborhood. The function $w$ then extends as a continuous function on $\bar{X}$ if and only if, for any $\bar{x} \in \overline{\mathrm{X}}$, $w(x)$ has a limit for $x \rightarrow \bar{x}$ in $\mathrm{X}$ (convergence of $w$ on the filter of traces on $\mathrm{X}$ of neighborhoods of $\bar{x}$ in $\overline{\mathrm{X}}$ ).

In the situation (8.2) (with $o \in \mathrm{V}$ for simplicity), one can treat $w$ as a multivalued function on $\mathrm{U}$. If $\bar{x}$ in $\bar{f}^{-1}(y)$ corresponds to the connected component $f^{-1}(\mathrm{~V})_{\sigma}$ of $f^{-1}(\mathrm{~V})$, the restriction of $w$ to $f^{-1}(\mathrm{~V})_{\sigma}$ is a multivalued function $w_{\sigma}$ on $\mathrm{V} \cap \mathrm{U}$. That $w(x)$ has a limit for $x \rightarrow \bar{x}$ means that there is $b$ in $\mathrm{B}$, and for each neighborhood $\mathrm{W}$ of $b$ a neighborhood $\mathrm{V}^{\prime}$ of $y$, such that all determinations of $w_{\sigma}(u)$, for $u \in \mathrm{V}^{\prime} \cap \mathrm{U}$, are in $\mathrm{W}$.

Example (8.4). - If we take for $\mathrm{Y}$ the unit disk $\mathrm{D} \subset \mathbf{C}$, for $\mathrm{U}$ the punctured unit disk $D^{*}$ and for $X$ the universal covering of $D^{*}$, the completion of $X$ over $D$ is deduced 
from $\mathrm{X}$ by adding onc point above $0: \overline{\mathrm{X}}=\mathrm{X} \cup\{0\}$. For $\lambda$ real $>0$, the function $z^{\lambda}$ has a limit for $z \rightarrow 0$ in $\mathrm{X}$, but it has no limit for non real $\lambda$ with $\mathscr{R} \lambda>0$. Similarly, $z . \log z$ has no limit.

(8.5) With the assumptions (8.2.I) and (8.2.2) on (Y,U), let $\mathscr{B}$ be the flat fiber space on $U$ defined by an action $\theta$ of $\pi_{1}(U, o)$ on a regular topological space $B$, and let $(\mathrm{X}, 0)$ be the smallest covering of $(\mathrm{U}, 0)$ on which $\mathscr{B}$ becomes trivial: one has $f^{-1}(0)=\pi_{1}(\mathrm{U}, 0) / \mathrm{Ker}(\theta)$. A continuous section $w$ of $\mathscr{B}$ defines by pull-back to $\mathrm{X}$ an equivariant continuous map from $\mathrm{X}$ to $\mathrm{B}$ (and conversely). We write $w$ for this map. When does it extend to $\overline{\mathrm{X}}$ ? Unravelling (8.3), we find that $\bar{w}$ has a limit for $x \rightarrow \bar{y}$ above $y$ if and only if there is a horizontal section $b$ of $\mathscr{B}$ over the trace on $U$ of a neighborhood $\mathrm{V} \in \mathscr{V}_{y}$ of $y$, toward which $w$ converges for $y^{\prime} \rightarrow y\left(y^{\prime} \in \mathrm{U}\right)$, in the following sense. For $y_{1} \in \mathrm{V} \cap \mathrm{U}$ and $\mathrm{W}_{1}$ a neighborhood of $b\left(y_{1}\right)$ in the fibre $\mathscr{B}_{y_{1}}$ of $\mathscr{B}$ at $y_{1}$, there is a neighborhood $\mathrm{V}^{\prime}$ of $y$ such that for any $y_{2} \in \mathrm{V}^{\prime} \cap \mathrm{U}$, and for $\mathrm{W}_{2}$ a neighborhood of $b\left(y_{2}\right)$ in the fiber of $\mathscr{B}$ at $y_{2}$, obtained by horizontal transport of $\mathrm{W}_{1}$ along any path in $\mathrm{V} \cap \mathrm{U}$ from $y_{1}$ to $y_{2}$, one has $w\left(y_{2}\right) \in \mathrm{W}_{2}$. This holds if $b$ has a system of horizontal neighborhoods $\mathrm{W}$ which are fiber arbitrarily small, such that $w\left(y_{1}\right)$ is in $\mathrm{W}$ for $y_{2}$ close enough to $y$. Of course, $b$ defines the limit of $w$.

In the rest of section 8 , the notation and assumptions $(4.0)$ as well as the assumption $\mathrm{N} \geqslant 4$ are in force.

(8.6) As in (3.8), fix a base point $o \in Q$, and let $\rho:(\widetilde{Q}, o) \rightarrow(Q, o)$ be the smallest covering of $(Q, o)$ on which the flat projective space bundle $B(\alpha)_{\mathbf{Q}}$ becomes trivial. For $Q \subset Y \subset Q_{\text {sat }}$ and $Y$ open in $Q_{\text {8st }}$, the composed map $\widetilde{Q} \rightarrow Y$ is a spread. We will write $\widetilde{Y}$ for the completion of $\widetilde{Q}$ over $Y$. The assumptions (8.2.I) and $(8.2 .2)$ are satisfied by $(Y, Q)$.

It follows from $(8.2 .4)$ that

$$
\begin{aligned}
& \pi_{1}(Q, 0) \text { acts on } \widetilde{Q}_{8 s t}, \\
& \pi_{1}(Q, 0) \backslash \widetilde{Q}_{B s t}=Q_{B s t} .
\end{aligned}
$$

Proposition (8.7). - The map $\widetilde{w}_{\mu}$ of (3.8) extends as a continuous map from $\widetilde{\mathrm{Q}}_{\mathrm{8st}}$ to the closed ball $\overline{\mathrm{B}(\alpha)_{0}^{+}}$, provided with the topology (5.4). The inverse image of $\mathrm{B}(\alpha)_{0}^{+}$consists of the stable points.

Proof. - We are in the situation envisioned in (8.5), if we take for $\mathscr{B}$ the flat fiber space with fiber $\overline{\mathrm{B}(\alpha)_{0}^{+}}$, completing $\mathrm{B}(\alpha)_{0}^{+}$. That the extendability criterion of $(8.6)$ applies is (6.8) for $y$ stable and (7.3) for $y$ semi-stable. Further, the limit occuring in criterion (8.5) lies in $\partial \mathrm{B}(\alpha)_{0}^{+}$if and only if $y$ is in $Q_{\text {eusp }}$.

The extended map $\widetilde{\mathrm{Q}}_{8 s t} \rightarrow \overline{\mathrm{B}(\alpha)}_{o}^{+}$is also denoted $\widetilde{w}_{\mu}$. It is $\pi_{1}(\mathrm{Q}, 0)$-equivariant. 
(8.8) For $T$ a stable partition of $S$ (cf. (6.9)), the system of the $Q_{T^{\prime}}$, for $T^{\prime}$ finer than $T$, is topologically constant along $Q_{T}$, and the inverse image $\widetilde{Q}_{T}$ of $Q_{T}$ in $\widetilde{Q}_{\text {st }}$ is hence a covering of $Q_{T}(\sim Q(T))$. By (6.9), on each connected component $\widetilde{Q}_{T}^{c}$ of $\widetilde{Q}_{T}$, the flat ball bundle $\mathrm{B}(\alpha(\mathrm{T}))^{+}$trivializes, and can be identified with a flat sub-ball bundle of the constant bundle $\mathrm{B}(\alpha)_{0}^{+}$, in such a way that

$$
\widetilde{w}_{\mu(T)}=\widetilde{w}_{\mu} \mid \widetilde{Q}_{T^{*}}^{e}
$$

From (3.9), we hence get:

Proposition (8.9). - For any component $\widetilde{\mathrm{Q}}_{\mathrm{T}}^{c}$ of $\widetilde{\mathrm{Q}}_{\mathrm{T}}, \widetilde{w}_{\mu} \mid \widetilde{\mathrm{Q}}_{\mathrm{T}}^{c}$ is an étale map from $\widetilde{\mathrm{Q}}_{\mathrm{T}}$ to a sub-ball of $\mathrm{B}(\alpha)_{0}^{+}$.

\section{Codimension I etaleness}

In this section, the notation and assumptions $(4.0)$ as well as the assumption $\mathrm{N} \geqslant 4$ are continued.

Let $\mathrm{T}$ be a stable partition of $\mathrm{S}$ (cf. (6.9)), with card $\mathrm{T}=$ card $\mathrm{S}-\mathrm{I}$; one coset of $\mathrm{T}$ has two elements and all others only one. Our aim in this section is to describe the flat bundle $B(\alpha)_{Q}$ over $Q$ (cf. (3.7)) and the holomorphic section $w_{\mu}$ in the neighborhood of a point in $Q_{\mathrm{T}} \subset \mathrm{Q}_{\mathrm{st}}$ (cf (6.9)).

(9.r) The subspace $Q_{T}$ of $Q_{\text {at }}$, defined in (6.9), is a locally closed purely one codimensional complex submanifold of the complex manifold $Q_{s t}$ and $Q \cup Q_{T}$ is open in $Q_{s t}$. The monodromy around $Q_{T}$ is the following conjugacy class in $\pi_{1}(Q)$.

Let $\varphi: D \rightarrow Q \cup Q_{T}$ be an embedding of the unit disc with $\varphi(D)$ transversal to $Q_{T}$ and $\varphi^{-1}\left(Q_{T}\right)=\{0\} ; \varphi$ induces an embedding of $D^{*}:=D-\{o\}$ in $Q$. The fundamental group $\pi_{1}\left(D^{*}\right)$ is canonically isomorphic to $Z$, generated by the loop $[0,1] \rightarrow D^{*}: t \mapsto z_{0} \cdot \exp (2 \pi i t)$. The decomposition group at $\varphi(0)$ is the image of $\pi_{1}\left(D^{*}\right)$ in $\pi_{1}(Q)$ and the monodromy around $Q_{T}$ is defined as the image of the positive generator of $\pi_{1}\left(D^{*}\right)$. Both are well defined up to conjugacy and independent of $\varphi$. Let us fix a base point $o$ in $Q$. The fundamental group $\pi_{1}(Q, o)$ acts on $B(\alpha)_{0}$. We will compute the action of the monodromy around $Q_{\mathrm{T}}$.

Let $\left\{s_{1}, s_{2}\right\}$ be the two element coset of $\mathrm{T}$ and choose additional elements $s_{3}, s_{4} \in \mathrm{S}$. We assume that $\mathbf{P}$ is the standard projective line $\mathbf{P}^{1}$, and we choose in it two distinct points $b, c \neq 0$. Let $\mathbf{M}_{0}$ be the space of injective maps $y: \mathbf{S} \rightarrow \mathbf{P}$ with $y\left(s_{1}\right)=0$, $y\left(s_{3}\right)=b, y\left(s_{4}\right)=c$. Let $\mathbf{M}_{0 \mathrm{~T}}$ be the space of maps $\mathbf{S} \rightarrow \mathbf{P}$ with $y(s)=y(t)$ if and only if $s$ and $t$ are in the same coset of $\mathrm{T}$, and $y\left(s_{1}\right)=y\left(s_{2}\right)=0, y\left(s_{3}\right)=b, y\left(s_{4}\right)=c$. The quotient map $M_{\text {st }} \rightarrow Q_{\text {st }}$ induces isomorphisms $\mathbf{M}_{0} \stackrel{\sim}{\rightarrow} Q, \quad M_{0 T} \stackrel{\sim}{\rightarrow} Q_{T}$ and $M_{0} \cup M_{0 T} \stackrel{\sim}{\rightarrow} Q \cup Q_{T}$. We will work on $M_{0} \cup M_{0 T}$, rather than on $Q \cup Q_{T}$. The monodromy around $Q_{T}$ is represented by the following loop in $M_{0}$ : it starts from a point $y_{0}$ with, for some $r, y_{0}\left(s_{2}\right)$ in the disc $\mathrm{D}_{r}$ of radius $r$ around o and the $y_{0}\left(s_{i}\right)(i \neq 1,2)$ 
outside of this disc; it is given by the map from $[0,1]$ to $\mathrm{M}_{0}, t \mapsto y_{t}$, with $y_{t}\left(s_{i}\right)$ constant for $i \neq 2$, and $y_{t}\left(s_{2}\right)=y_{0}\left(s_{2}\right) \cdot \exp (2 \pi i t)$.

Above this loop, one can construct on $\mathrm{P}_{\mathrm{M}_{\mathbf{a}}}$ a local system $\mathrm{L}$ with fiberwise monodromy $\alpha$ as follows: we fix a base point $d$ on $\mathrm{P}$, outside of $y_{0}(\mathrm{~S})$ and of $\mathrm{D}_{r}$, and take for $\mathrm{L}$ the local system trivialized on the constant section $d$ and with the required monodromy. The action of monodromy on $\mathrm{B}(\alpha)_{y_{0}}=\mathrm{PH}^{1}\left(\mathrm{P}_{y_{0}}, \mathrm{~L}\right)$ is deduced from the action of monodromy on $\mathrm{H}^{1}\left(\mathrm{P}_{y_{0}}, \mathrm{~L}\right)$.

Proposition (9.2). - In a suitable basis of $\mathrm{H}^{1}\left(\mathrm{P}_{y_{0}}, \mathrm{~L}\right)$, the above action of the monodromy around $\mathrm{Q}_{\mathrm{T}}$ is given by a diagonal matrix $\operatorname{diag}\left(\alpha_{1} \alpha_{2}, \mathrm{I}, \ldots, \mathrm{I}\right)$.

Proof. - Set $\mathrm{S}_{1}=\left\{s_{1}, s_{2}\right\}$ and $\mathrm{S}_{2}=\mathrm{S}-\mathrm{S}_{1}$. Let $\mathrm{T}_{1}, \mathrm{~T}_{2}$ be trees as in $(2.5)$ with the vertices of $T_{i}$ in $S_{i}(i=1,2)$ and let $\beta: T_{1} \cup T_{2} \rightarrow P$ be an embedding with $\beta \mid \mathrm{S}=y_{0}$. We may and shall assume that $\beta \mid \mathrm{T}_{1}$ is the straight line segment from $y_{0}\left(s_{1}\right)=0$ to $y_{0}\left(s_{2}\right)$ and that $\beta\left(\mathrm{T}_{2}\right)$ is outside of the disc $\mathrm{D}_{r}$. For each (open) cdgc $a$ of $T_{1}$ or $T_{2}$, we choose one of its orientations, and a non zero section $\ell(a)$ of $\beta^{*} \mathrm{~L}$ above it. As in (2.4), (2.5), each edge then defines a closed L-valued current $\ell(a) \beta(a)$, and the cohomology classes of those currents form a basis of $\mathrm{H}^{1}\left(\mathrm{P}_{v_{1}}, \mathrm{~L}\right)$. It is the basis we will use.

To transport our basis elements around the loop $y_{t}(0 \leq t \leq \mathrm{I})$ horizontally, it suffices to deform $\beta$ with $\beta_{t} \mid \mathrm{S}=y_{t}$, dragging along the $\ell(a)$. We will keep $\beta_{l} \mid \mathrm{T}_{2}$ fixed and take for $\beta_{t} \mid T_{1}$ the family of straight line segments from $y_{t}\left(s_{1}\right)=0$ to $y_{t}\left(s_{2}\right)=y_{0}\left(s_{2}\right) \cdot \exp (2 \pi i t)$. Outside of $\mathrm{D}_{r} \subset \mathbf{P}$, the fibers $\mathrm{P}_{y}$ and $\mathrm{L}$ remain constant, and the $\ell(a) \cdot \beta_{1} \mid a$ for $a$ an edge of $T_{2}$ are, as currents, independent of $t$. It follows that the monodromy is trivial on the $\ell(a) \cdot \beta \mid a$ for $a$ an edge of $T_{2}$. Let $a$ be the unique edge of $T_{1}$. When $\beta_{t}(a)(0 \leqslant t \leqslant \mathrm{I})$ has made a complete turn around $o, \ell(a)$ is multiplied by $\alpha_{1} \alpha_{2}$. Indeed, if $p_{t}(x):[0,1] \rightarrow \mathbf{P}$ is a path from the base point $d$ on $\mathbf{P}$ to a point $d_{l}$ on $\beta_{l}(a)$, and if this path deforms with $t$ so as to always avoid $\beta_{l}(S)$, one can take $\ell(a)$ to be obtained at $d_{l}$ by transporting along $p_{t}$ the trivialising section $e$ of $\mathrm{L}$ at $d$. As the following picture shows, $p_{1}$ can be chosen to differ from $p_{0}$ by a loop around $y_{0}\left(s_{1}\right)$ and $y_{0}\left(s_{2}\right)$. Around this loop, L has monodromy $\alpha_{1} \alpha_{2}$, and the claim follows.

$t=0:$

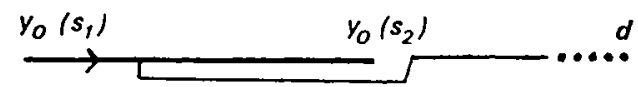

$t$ small:

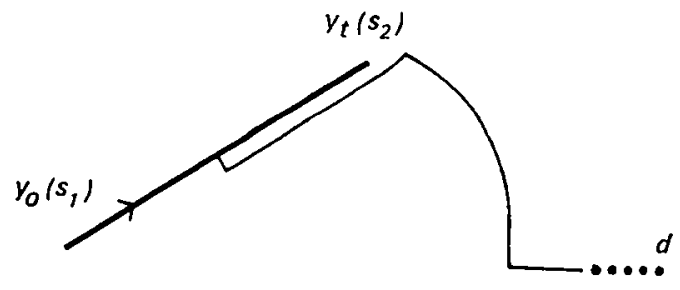


$t=\mathrm{I}:$

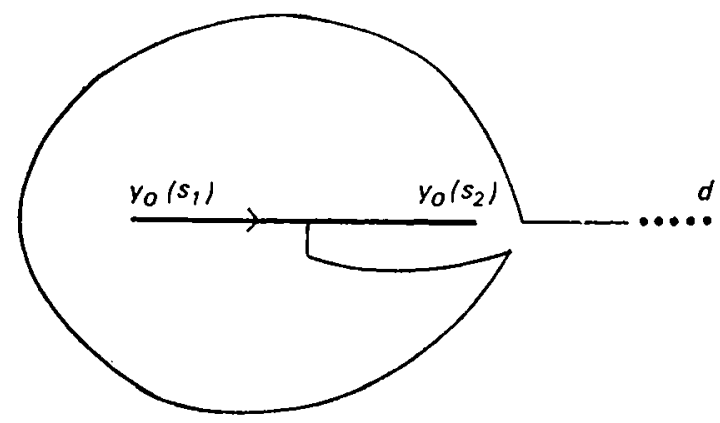

Remark (9.3). - In the computation (9.2), we have made use of the assumption $\mu_{1}+\mu_{2}<\mathrm{I}$ only in the weaker form $\alpha_{1} \alpha_{2} \neq \mathrm{I}$. In the case $\mu_{1}+\mu_{2}>\mathrm{I}$, the monodromy when $s_{2}$ turns around $s_{1}$ would be given by the same formula, but the basis $\mathbf{e}$ in which it holds would have $\left(e_{1}, e_{1}\right)>0$ instead of $\left(e_{1}, e_{1}\right)<0$ : it would be a pseudoreflection with respect to a hyperplane not meeting the ball $\mathrm{B}(\alpha)_{y_{0}}^{+}$.

More preciscly, one checks that if the section $\ell(a)$ of $\mathrm{L}$, for $a$ the only edge of $\mathrm{T}_{1}$, is chosen of length one: $(\ell(a), \ell(a))=\mathrm{I}$, then

$$
\left(e_{1}, e_{1}\right)=\frac{-\mathrm{I}}{2 \pi i} \cdot \frac{\mathrm{I}}{2} \cdot\left(\frac{\alpha_{1}+\mathrm{I}}{\alpha_{1}-\mathrm{I}}+\frac{\alpha_{2}+\mathrm{I}}{\alpha_{2}-\mathrm{I}}\right) .
$$

We will not necd this formula.

Remark (9-4). - For later computations, it will be more convenient to work with the basis of the dual space $\mathrm{H}_{1}^{\mathrm{L}}\left(\mathrm{P}-y_{0}(\mathrm{~S}), \mathrm{L}^{\mathrm{V}}\right)$ to $\mathrm{H}^{\mathbf{1}}\left(\mathrm{P}-y_{0}(\mathrm{~S}), \mathrm{L}\right)$ given by a choice of non zero sections $\ell^{\prime}(a)$ of $\beta^{*} L^{v}$ above the edges of $T_{1}$ or $T_{2}$. The same arguments as in (9.2), show that on such a basis of the dual space, monodromy around $Q_{T}$ acts by $\operatorname{diag}\left(\left(\alpha_{1} \alpha_{2}\right)^{-1}, \mathrm{I}, \ldots, \mathrm{I}\right)$.

(9.5) Fix $y \in Q_{\mathrm{T}}$. We will describe the section $w_{\mu}$ of $\mathbf{B}(\alpha)_{Q}$ near $y$. A neighborhood of $y$ in $Q \cup Q_{T}$ and local coordinates are chosen as follows. First, one replaces $\mathrm{Q} \cup \mathrm{Q}_{\mathrm{T}}$ by $\mathrm{M}_{0} \cup \mathrm{M}_{\mathrm{0T}}$, as in (9.I). It is convenient to choose $b$ and $c$, in the definition of $\mathrm{M}_{0}$, so that $\infty \notin y(\mathrm{~S})$ and that the discs of radius $\mathrm{I}$ around the points in $y(\mathrm{~S})$ are disjoint. We do so. The chosen neighborhood $U$ is then identified with the space of $m \in \mathrm{M}_{\mathbf{0}} \cup \mathrm{M}_{\mathbf{0 T}}$ such that $|m(s)-y(s)|<\mathrm{I}$. The chosen coordinates are the $m(s)-y(s) \quad\left(s \neq s_{1}, s_{3}, s_{4}\right)$. If $\mathrm{S}^{\prime}:=\mathrm{S}-\left\{s_{1}, s_{2}, s_{3}, s_{4}\right\}$, this system of coordinates identifies $\mathrm{U}$ with $\mathrm{D} \times \mathrm{D}^{\mathrm{s}^{\prime}}$, and $\mathrm{U} \cap \mathrm{M}_{\mathbf{0}}$ with $\mathrm{D}^{*} \times \mathrm{D}^{\mathrm{S}^{\prime}}$.

We choose $\mathrm{L}$ as in (9. I), using a base point $d$ on $\mathrm{P}$ at distance $>\mathrm{I}$ from $y(\mathrm{~S})$-for instance $d$ a large positive real number. In $\mathrm{U} \cap \mathrm{M}_{0}$, we choose a base point $m_{0}$-for instance with $m_{0}\left(s_{2}\right)=\frac{\mathrm{r}}{2}$ and $m_{0}(s)=y(s)$ for $s \neq s_{2}$. We view $w_{\mu}$ as a multivalued map from $\mathrm{U} \cap \mathrm{M}_{0}$ to $\mathrm{B}(\alpha)_{m_{0}}=\mathrm{PH}^{1}\left(\mathrm{P}-m_{0}(\mathrm{~S}), \mathrm{L}\right)$, and on $\mathrm{H}^{1}\left(\mathrm{P}-m_{0}(\mathrm{~S}), \mathrm{L}\right)$ we 
use the coordinates provided by a basis (9.4) of the dual space. The multivalued map $w_{\mu}$ then lifts to the multivalued map with values in $\mathbf{C}^{N-3}$ having as coordinates (up to multiplicative constants)

$$
\int_{0}^{m\left(s_{2}\right)} z^{-\mu_{1}}\left(z-m\left(s_{2}\right)\right)^{-\mu_{2}} \cdot \prod_{s \neq s_{1}, s_{2}}(z-m(s))^{-\mu_{s}} \cdot d z
$$

as first coordinate and as the other coordinates the integrals

$$
\int \Pi(z-m(s))^{-u_{s}} d z
$$

taken along a path remaining outside the disc $|z|<\mathrm{I}$ from one $m(s)$ to another $\left(s \neq s_{1}, s_{2}\right)$. When $m\left(s_{2}\right)$ turns once around $m\left(s_{1}\right)$, the first coordinate gets multiplied by $\left(\alpha_{1} \alpha_{2}\right)^{-1}=\exp \left(2 \pi i\left(1-\mu_{1}-\mu_{2}\right)\right)$ while the others regain their value $(9 \cdot 4)$. This allows us to write the first coordinate as $\left(m\left(s_{2}\right)-m\left(s_{1}\right)\right)^{1-\mu_{1}-\mu_{2}} \cdot I(m)$, with $I(m)$ an ordinary function on $\mathrm{U} \cap \mathrm{M}_{0}$. When $m$ tends to $m_{1} \in \mathrm{M}_{\mathrm{OT}}, \mathrm{I}(m)$ tends to the limit

$$
\prod_{s \neq s_{1}, s_{2}}(-m(s))^{-\mu_{s}} \cdot \int_{0}^{1} z^{-\mu_{1}}(z-\mathrm{I})^{-\mu_{2}} d z \text {, }
$$

which is non zero (2.1 7.I), hence $I(m)$ extends as a holomorphic function on $U$, invertible on $\mathrm{U} \cap \mathrm{M}_{\mathbf{0 T}}$. The other coordinates extend as holomorphic functions on $\mathrm{U}$, having as restriction to $\mathrm{U} \cap \mathrm{M}_{0 \mathrm{~T}}$ integrals

$$
\int z^{-\mu_{1}-\mu_{2}} \cdot \prod_{s \neq s_{1}, s_{2}}(z-m(s))^{-\mu_{s}} \cdot d z
$$

By (3.9) applied to $\mu_{T}$, these functions on $U \cap M_{0 T}$ are the projective coordinates of an etale map from $U \cap M_{0 T}$ to the projective $[(N-1)-3]$-space (cf. (8.9)).

(9.6) In suitable local coordinates, the multivalued map $w_{\mu}$ from $\mathrm{U} \cap \mathrm{M}_{0}$ to $\mathrm{B}(\alpha)_{m_{0}}$ has the form

$$
\left(z_{0}, \mathbf{z}\right) \mapsto\left(z_{0}^{1-\mu_{1}-\mu_{2}} \mathrm{I}\left(z_{0}, \mathbf{z}\right)\right), \mathrm{J}\left(z_{0}, \mathbf{z}\right) ;
$$

here, $\left(z_{0}, \mathbf{z}\right)$ are local coordinates on $\mathrm{U}$ coming from the product decomposition above, $\mathrm{U} \sim \mathrm{D} \times \mathrm{D}^{n}, \mathrm{U} \cap \mathrm{M}_{\mathbf{0}} \sim \mathrm{D}^{*} \times \mathrm{D}^{n}$; the local coordinates on the image of $\mathrm{U}$ in $\mathrm{B}(\alpha)_{m_{0}}$ are selected on an open set $\mathrm{V}$ with $\mathrm{V} \sim \mathrm{D} \times \mathrm{D}^{n}$ and $\{0\} \times \mathrm{D}^{n}$ lying in a hyperplane; and finally, $\mathrm{I}: \mathrm{D} \times \mathrm{D}^{n} \rightarrow \mathbf{C}$ and $\mathrm{J}: \mathrm{D} \times \mathrm{D}^{n} \rightarrow \mathrm{D}^{n}$ are holomorphic maps, with $\mathrm{I}(\mathbf{o}, \mathbf{z}) \neq 0$ and $\mathrm{J}(\mathrm{o}, \mathbf{z}): \mathrm{D}^{n} \rightarrow \mathrm{D}^{n}$ etale.

At a point of $\{0\} \times \mathrm{D}^{n} \subset \mathrm{D} \times \mathrm{D}^{n}$, the holomorphic functions $z_{0} . \mathrm{I}\left(z_{0}, \mathbf{z}\right)^{1 /\left(1-\mu_{1}-\mu_{2}\right)}$ and $\mathrm{J}\left(z_{0}, \mathbf{z}\right)_{i}(\mathbf{1} \leq i \leq n)$ form a system of coordinates. In those coordinates, the multivalued map $w_{\mu}$ is

$$
\left(z_{0}, z_{1}, \ldots, z_{n}\right) \rightarrow\left(z_{0}^{1-\mu_{1}-\mu_{2}}, z_{1}, \ldots, z_{n}\right) .
$$

Suppose $I-\mu_{1}-\mu_{2}$ is rational, set $I-\mu_{1}-\mu_{2}=\ell / k$, in reduced terms. If $\widetilde{\mathrm{D}}$ is another copy of the disc, with coordinate $u$, mapping to $\mathrm{D}$ by $u \mapsto u^{k}$, 
the pull back to $\widetilde{\mathrm{D}} \times \mathrm{D}^{n}$ of the multivalued map above is the holomorphic map $\left(u, z_{1}, \ldots, z_{n}\right) \mapsto\left(u^{\ell}, z_{1}, \ldots, z_{n}\right)$. This map is etale in a neighborhood of

$$
\{0\} \times \mathrm{D}^{n} \subset \tilde{\mathrm{D}} \times \mathrm{D}^{n}
$$

if and only if $\ell=I$, i.e. if and only if $\left(1-\mu_{1}-\mu_{2}\right)^{-1}$ is an integer.

(9.7) Let $\mathrm{Y}$ be a complex analytic manifold, $\mathscr{E}$ a family of disjoint closed complex analytic submanifolds purely of complex codimension $\mathrm{I}, \mathrm{Z}=\amalg\{\mathrm{H} ; \mathrm{H} \in \mathscr{G}\}$, and $\kappa: \mathscr{F} \rightarrow \mathbf{N}$ a positive integer valued function.

A continuous map $\psi: R \rightarrow Y$ is called a branched cover with branch locus $Z$ of order $\mathrm{K}$ if and only if:

1. $\psi$ induces a covering map from $\psi^{-1}(\mathrm{Y}-\mathrm{Z})$ to $\mathrm{Y}-\mathrm{Z}$.

2. For any $\mathrm{H} \in \mathscr{E}$, if we put $k:=\kappa(\mathrm{H})$, any $y \in \mathrm{H}$ admits a neighborhood $\mathrm{V}$ in $Y$ such that the restriction of $\psi$ to any connected component of $\psi^{-1}(V)$ is topologically equivalent to the map

$$
\mathrm{D}^{n} \rightarrow \mathrm{D}^{n}:\left(z_{1}, \ldots, z_{n}\right) \mapsto\left(z_{1}^{k}, z_{2}, \ldots, z_{n}\right) .
$$

A branched cover $\psi: R \rightarrow Y$ has a unique complex analytic manifold structure such that $\psi$ is holomorphic.

(9.7.1) Let $\psi: \mathrm{R} \rightarrow \mathrm{Y}$ be a branched cover with branch locus $\mathrm{Z}$. It follows from the definitions that $\psi$ is the completion of $\psi^{-1}(\mathrm{Y}--\mathrm{Z}) \rightarrow \mathrm{Y}$ over $\mathrm{Y}$. Moreover, given any commutative diagram

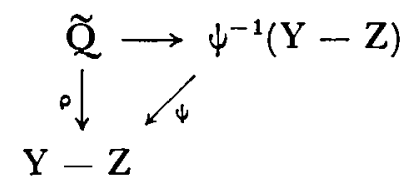

with $\rho$ and $\psi$ spreads, then there is a diagram

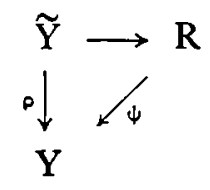

with $\rho$ and $\psi$ the completions of the spreads above, which is also commutative by the universal property of completions (8. I. I).

(9.8) Let $Q$ be an open set in a locally connected space $Y$. Let $\rho: \widetilde{Q} \rightarrow Q$ be a spread and denote by $p$ also the completion $\widetilde{Y} \rightarrow Y$ of $\rho$. Then for any open set $U$ in $Y$, the restriction of $\rho$ to a connected component $\rho^{-1}(U)^{c}$ of $\rho^{-1}(U)$ is the completion of the spread $p^{-1}(U)^{c} \cap p^{-1}(U \cap Q) \rightarrow U \cap Q$. 
(9.9) We now assume that the $\mu_{s}$ are rational (cf. (2. I2)). Let $\mathscr{F}_{1}$ denote the family of stable partitions $\mathrm{T}$ of $\mathrm{S}$ such that $\operatorname{card} \mathrm{T}=\operatorname{card} \mathrm{S}-\mathrm{I}$. For $\mathrm{T}$ in $\mathscr{E}_{1}$, and $\{s, t\}$ the coset of $\mathrm{T}$ with two elements, let $k_{\mathrm{T}}$ be the denominator of $1-\mu_{s}-\mu_{l}$. Set

$$
\begin{aligned}
\mathrm{Q}_{1} & =\mathrm{Q} \cup \underset{\mathrm{T} \in \mathscr{\sigma}_{1}}{\amalg} \mathrm{Q}_{\mathrm{T}}, \\
\overline{\mathrm{Q}}_{1} & =\text { completion of } \widetilde{Q} \text { over } \mathrm{Q}_{1}, \\
\kappa\left(\mathrm{Q}_{\mathrm{T}}\right) & =k_{\mathrm{T}} \text { for each } \mathrm{T} \in \mathscr{G}_{1} .
\end{aligned}
$$

Proposition (9.10). - The map $\widetilde{Q}_{1} \rightarrow Q_{1}$ is a branched cover with branch locus $\mathrm{U} \mathrm{Q}_{\mathrm{T}}$ of order $\kappa$.

By (9.8), the problem is local around each $y \in Q_{\mathrm{T}}$. The claim then results from (9.2) and the fact that the order in the projective unitary group of $\operatorname{diag}\left(\alpha_{b} \alpha_{l}, I, \ldots, I\right)$ is the denominator of $I-\mu_{s}-\mu_{i}$.

Proposition (9.1工). - The map $\widetilde{w}_{\mu}: \widetilde{\mathrm{Q}}_{1} \rightarrow \mathrm{B}^{+}(\alpha)_{0}$ is holomorphic.

For $\widetilde{w}_{\mu}$ is holomorphic on $\widetilde{Q}$ and is continuous on $\widetilde{Q}_{1}$ by Proposition (8.7). Since $\widetilde{Q}_{1}-\widetilde{Q}$ has $\mathbf{C}$-codimension $I$, the assertion follows from the theorem on removable singularities.

Proposition (9.12). - Assume that

INT $\quad 0<\mu_{s}<\mathrm{I}, \Sigma \mu_{\mathrm{s}}=2$ and for all $s, t \in \mathrm{S}$ distinct and with $\mu_{\mathrm{s}}+\mu_{\mathrm{t}}<\mathrm{I}$, $\left(\mathrm{I}-\mu_{\mathrm{s}}-\mu_{l}\right)^{-1}$ is an integer.

Then, $\widetilde{w}_{\mu}: \widetilde{\mathrm{Q}}_{1} \rightarrow \mathrm{B}^{+}(\alpha)_{0}$ is etale.

This follows from the local description (9.6).

\section{Proof of discreteness}

In this section, the notation and assumptions $(4.0)$ as well as the assumption $\mathrm{N} \geqslant 4$ are continued.

(10.1) We will give two proofs that when condition INT is satisfied, the image $\Gamma$ of $\pi_{1}(Q, 0)$ in $P U(1, N-2)$ is discrete.

The first is shorter, but relies on a detailed local analysis of analytic varieties and holds only when $Q_{s t}=Q_{s s t}$. The second is longer but more elementary-and eventually more powerful.

(10.2) First proof. We follow the strategy explained in (2.13). The key point in proving that the projection $\widetilde{Q}_{\mathrm{at}} \rightarrow \mathrm{Q}_{\mathrm{st}}$ is locally (on $\widetilde{Q}_{\mathrm{st}}$ ) finite-to-one is the following lemma. 
Lemma (ro.3). - Let $\mathrm{D}_{1}, \mathrm{D}_{2}$ and $\mathrm{D}_{3}$ be three distinct lines in $\mathbf{C}^{2}$ passing through 0 , and $n_{1}, n_{2}, n_{3}$ integers $>$ I such that $\frac{\mathrm{I}}{n_{1}}+\frac{\mathrm{I}}{n_{2}}+\frac{\mathrm{I}}{n_{3}}>\mathrm{I}$. Fix a base point $b \in \mathbf{C}^{2}-\left(\mathrm{D}_{1} \cup \mathrm{D}_{2} \cup \mathrm{D}_{3}\right)$, and consider the coverings of $\left(\mathbf{G}^{2}-\left(\mathrm{D}_{1} \cup \mathrm{D}_{2} \cup \mathrm{D}_{3}\right), b\right)$, with ramification index along $\mathrm{D}$ dividing $n_{i}(i=1,2,3)$ : the monodromy permutation of the sheets, when turning around $\mathrm{D}_{i}$, is of order dividing $n_{i}$. Then the universal such covering is a finite covering.

Proof. - There exists a spherical triangle $\Delta$ with angles $\frac{\pi}{n_{1}}, \frac{\pi}{n_{2}}$ and $\frac{\pi}{n_{3}}$. Let $\mathrm{W}$ be the Coxeter group generated by the reflections along its edges. The $w \Delta(w \in W)$ form a tessellation of the sphere, and hence

$$
|\mathrm{W}|=\operatorname{area}\left(\mathrm{S}^{2}\right) / \operatorname{area}(\Delta)=4\left(\frac{\mathrm{I}}{n_{1}}+\frac{\mathrm{I}}{n_{2}}+\frac{\mathrm{I}}{n_{3}}-\mathrm{I}\right)^{-1} \text {. }
$$

Let $\mathrm{W}^{+}$be the subgroup of index two in $\mathrm{W}$ consisting of the orientation preserving elements. If we identify the sphere with the Riemann sphere metrized by its Fubini metric, $\mathrm{W}^{+}$becomes a finite subgroup of $\mathrm{PU}(2)$, of order

$$
d=2\left(\frac{\mathrm{I}}{n_{1}}+\frac{\mathrm{I}}{n_{2}}+\frac{\mathrm{I}}{n_{3}}-\mathrm{I}\right)^{-1} .
$$

The quotient $\mathbf{P}^{1} / \mathrm{W}^{+}$is of genus $\mathbf{o}$ and hence is a projective line. Fix an isomorphism $\mathbf{P}^{1} / \mathbf{W}^{+} \sim \mathbf{P}^{1}$ and let $f: \mathbf{P}^{1} \rightarrow \mathbf{P}^{1} / \mathrm{W}^{+} \sim \mathbf{P}^{1}$ be the quotient map. The pull-back by $f$ of the line bundle $\mathcal{O}(-\mathrm{I})$ on $\mathbf{P}^{1}$ is of degree the negative of $d=\operatorname{deg}(f)=\left|\mathrm{W}^{+}\right|$. Fix an isomorphism $\mathcal{O}(-\mathrm{I})^{\otimes d} \stackrel{\sim}{\rightarrow} f^{*} \mathcal{O}(-\mathrm{I})$. The group $\mathrm{W}^{+}$acts naturally on $\left(\mathbf{P}^{1}, f^{*} \mathcal{O}(-\mathrm{I})\right) \sim\left(\mathbf{P}^{1}, \mathcal{O}(-\mathrm{I})^{\otimes d}\right)$. Let $\mathrm{H}$ be the group of all automorphisms $(h, \varepsilon)$ of $\left(\mathbf{P}^{1}, \mathcal{O}(-\mathrm{I})\right)$ with $\left(h, \varepsilon^{\otimes d}\right) \in \mathrm{W}^{+}$. It is a central extension of $\mathrm{W}^{+}$by $\mu_{d}$, the group of roots of unity of order dividing $d$. As a space, the line bundle $\mathcal{O}(-\mathrm{I})$, minus the o section, is $\mathbf{C}^{2}-\{o\}$, the bundle map being the natural projection $\mathbf{C}^{2}-\{o\} \rightarrow \mathbf{P}^{1}$. The action of $\mathbf{H}$ on $\mathbf{C}^{2}-\{0\}$ is induced by a linear action on $\mathbf{C}^{2}$ : the contragredient of its action on $\mathrm{H}^{0}\left(\mathbf{P}^{1}, \mathcal{O}(\mathrm{I})\right)$. The quotient map of the space $\left(\mathbf{P}^{1}, \mathcal{O}(-\mathrm{I})\right)$ to its $\mu_{d}$ orbits may be identified with a $d$-th power map of $\left(\mathbf{P}^{1}, \mathcal{O}(-\mathrm{I})\right)$ to $\left(\mathbf{P}^{1}, \mathcal{O}(-\mathrm{I})^{\otimes d}\right)$, and the quotient map $g: \mathbf{C}^{2}-o$ to its $\mathbf{H}$ orbits is the composite of the $d$-th power map to the total space of $f^{*} \mathcal{O}(-\mathrm{I})$ minus its zero section, followed by the quotient map to $\mathrm{W}^{+}$ orbits. Thus the quotient maps by $\mathrm{H}$ and $\mathrm{W}^{+}$give rise to a commutative diagram

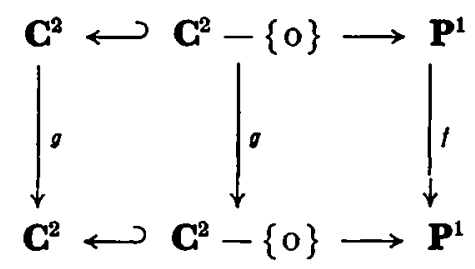


The stability groups in $\mathrm{W}^{+}$of the vertices $p_{1}, p_{2}, p_{3}$, of the original spherical triangle are cyclic of order $n_{1}, n_{2}, n_{3}$, and the points in the orbits of $p_{1}, p_{2}, p_{3}$ are the only points with a non trivial stabilizer (this results from the fact that the stabilizer in $W$ of any point of $\Delta$ is generated by the reflections along the edges passing through the point ([4] V (3.3) prop. I), or simply from the fact that $\Delta$ is a fundamental domain for $W([20]))$. Let $d_{i}=f\left(p_{i}\right)$ and $\mathrm{D}_{i} \subset \mathbf{C}^{2}$ be the corresponding line. One easily checks that $g$ ramifies only along $D_{1}, D_{2}, D_{3}$ and that at each point above a point of $D_{i}-\{0\}$, the ramification index is exactly $n_{i}$. If $X$ is a covering of $C^{2}-\left(D_{1} \cup D_{2} \cup D_{3}\right)$ as in (10.3), its pullback by $g$ can hence be extended to unbranched covering of $\mathbf{C}^{2}-\{0\}$. As $\mathbf{C}^{2}-\{0\}$ is simply connected, each connected component of this covering of $\mathbf{C}^{2}-\{0\}$ is trivial. This implies that the universal covering of type (10.3) is

$$
g^{-1}\left(\mathbf{C}^{2}-\left(\mathrm{D}_{1} \cup \mathrm{D}_{2} \cup \mathrm{D}_{3}\right)\right) \rightarrow \mathbf{C}^{2}-\left(\mathrm{D}_{1} \cup \mathrm{D}_{2} \cup \mathrm{D}_{3}\right)
$$

It is finite.

Remark (10.4). - (i) The proof showed that the universal covering (10.3) is of order $\left[2\left(\frac{\mathrm{I}}{n_{1}}+\frac{\mathrm{I}}{n_{2}}+\frac{\mathrm{I}}{n_{3}}-\mathrm{I}\right)^{-1}\right]^{2}$. It is a Galois covering, with group a central extension of $\mathrm{W}^{+}$by a cyclic group of order $\left|\mathrm{W}^{+}\right|$.

(ii) If $\mu_{1}+\mu_{2}=\mathrm{I}-\frac{\mathrm{I}}{n_{3}}, \mu_{2}+\mu_{3}=\mathrm{I}-\frac{\mathrm{I}}{n_{1}}$ and $\mu_{3}+\mu_{1}=\mathrm{I}-\frac{\mathrm{I}}{n_{2}}$, one has $\mathrm{I}-\mu_{1}-\mu_{2}-\mu_{3}=\frac{\mathrm{I}}{2}\left(\frac{\mathrm{I}}{n_{1}}+\frac{\mathrm{I}}{n_{2}}+\frac{\mathrm{I}}{n_{3}}-\mathrm{I}\right)$. The condition $\frac{\mathrm{I}}{n_{1}}+\frac{\mathrm{I}}{n_{2}}+\frac{\mathrm{I}}{n_{3}}>\mathrm{I}$ amounts to $\mu_{1}+\mu_{2}+\mu_{3}<I$, and when it holds $\left(I-\mu_{1}-\mu_{2}-\mu_{3}\right)^{-1}=\left|W^{+}\right|$is an integer, as observed in (6.10).

(iii) The fundamental group of $\mathbf{C}^{2}-\left(\mathrm{D}_{1} \cup \mathrm{D}_{2} \cup \mathrm{D}_{3}\right)$ is generated by three elements $\gamma_{1}, \gamma_{2}, \gamma_{3}\left(\gamma_{i}\right.$ conjugate to a small positive loop around $\left.D_{i}\right)$ with relations those expressing that $\gamma_{1} \gamma_{2} \gamma_{3}$ (conjugate to a small loop around o, on a general line D through 0 ) is central. To get $\mathrm{H}$, one imposes the additional relations $\gamma_{i}^{n_{i}}=1$.

(iv) In the commutative diagram

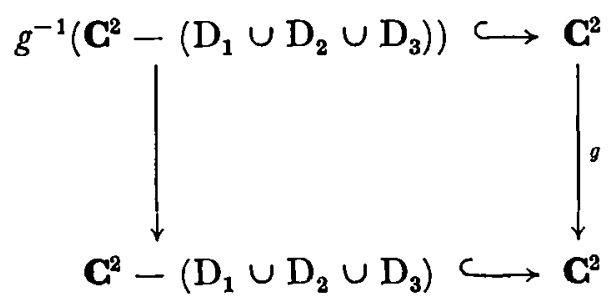

$\mathbf{C}^{2}$, at the upper right corner, is the completion of $g^{-1}\left(\mathbf{C}^{2}-\left(\mathbf{D}_{1} \cup \mathbf{D}_{2} \cup \mathrm{D}_{3}\right)\right)$ over $\mathbf{C}^{2}$-at points of $\mathbf{C}^{2}-\{0\}$ by $(9.7 . I)$ and at $\mathbf{o}$ by direct verification. Such a fact is generally true for a finite morphism $g$ of normal analytic spaces. The completion of $g^{-1}\left(\mathbf{C}^{2}-\left(\mathrm{D}_{1} \cup \mathrm{D}_{2} \cup \mathrm{D}_{3}\right)\right)$ over $\mathbf{C}^{2}$ thus acquires a structure of a normal analytic 
space-indeed of a non-singular analytic manifold; this structure is in fact the unique one for which the projection to $\mathbf{C}^{2}$ is analytic.

Remark (ro.5). - Let $\mathrm{J}=\mathrm{J}^{\prime} \sqcup \mathrm{J}^{\prime \prime} \cup \mathrm{J}^{\prime \prime \prime}$ be a finite index sct. For $j \in \mathrm{J}^{\prime}$, let $n_{1}(j), n_{2}(j), n_{3}(j)$ satisfy $\frac{\mathrm{I}}{n_{1}(j)}+\frac{\mathrm{I}}{n_{2}(j)}+\frac{\mathrm{I}}{n_{3}(j)}>\mathrm{I} ;$ for $j \in \mathrm{J}^{\prime \prime}$, let $n(j)$ be any
integer $\geq \mathrm{I}$. We define

$$
\begin{aligned}
& \mathrm{X}(j)=\mathbf{C}^{2}-\mathrm{D}_{1}-\mathrm{D}_{2}-\mathrm{D}_{3} \subset \mathbf{C}^{2} \quad \text { for } j \in \mathrm{J}^{\prime}, \\
& \mathbf{C}-\{0\} \quad C \mathbf{C}^{2} \text { for } j \in \mathrm{J}^{\prime \prime} \text {, } \\
& \mathbf{C} \text { for } j \in \mathrm{J}^{\prime \prime \prime} \text {. }
\end{aligned}
$$

The product $\mathrm{X}$ of the $\mathrm{X}(j)$ is the complement of a divisor in $\mathrm{Y}:=\left(\mathbf{C}^{2}\right)^{\mathbf{J}^{\prime}} \times \mathbf{C}^{\mathrm{J}^{\prime \prime}} \mathrm{I} \mathbf{J}^{\prime \prime \prime}$. The covering $\widetilde{\mathrm{X}}$ of $\mathrm{X}$, with ramification index along $\operatorname{pr}_{j}^{-1}\left(\mathrm{D}_{i}\right)$ dividing $n_{i}(j), \quad\left(j \in \mathrm{J}^{\prime}\right)$, with ramification index along $\operatorname{pr}_{j}^{-1}(\{o\})$ dividing $n(j)\left(j \in \mathrm{J}^{\prime \prime}\right)$, and universal with respect to those properties, is the product of the corresponding coverings of each $\mathrm{X}(j)$. Hence $\widetilde{X}$ is a finite covering of $X$. The completion $\tilde{Y}$ of $\tilde{X}$ over $\mathrm{Y}$ is similarly a product. By (10.4) (iv), $\widetilde{Y}$ has a unique structure of a normal (in fact non-singular) analytic space for which the map to $Y$ is analytic. If $\tilde{X}_{1}$ is a covering space of $X$ and a quotient of $\tilde{X}$, then $\widetilde{X}_{1}=\tilde{X} / G$ with $G$ a finite group, and the completion $\widetilde{Y}_{1}$ of $\widetilde{X}_{1}$ over $Y$ is $\widetilde{Y} / G$. It inherits the structure of a normal analytic space from $\widetilde{Y}$.

Similar remarks apply with $\mathrm{X}$ replaced by the trace on $\mathrm{X}$ of a ball around $\mathrm{o}$ in $\left(\mathbf{C}^{2}\right)^{\mathrm{J}^{\prime}} \times \mathbf{C}^{\mathrm{J}^{\prime \prime} \mathrm{L} \mathrm{J}^{\prime \prime \prime}}$.

Lemma (10.6). - If condition (INT) is satisfied, $\widetilde{\mathrm{Q}}_{\mathrm{st}}$ admits a structure of normal analytic space, such that each $y_{0} \in \mathcal{Q}_{\mathrm{st}}$ has an open neighborhood $\mathrm{U}$ whose inverse image in $\widetilde{\mathrm{Q}}_{\mathrm{st}}$ is a disjoint sum of finite ramified coverings of $\mathrm{U}$.

It suffices to show that each $y_{0} \in \widetilde{Q}_{s t}$ has an open neighborhood $\mathrm{U}$ whose inverse image in $\widetilde{Q}_{\text {st }}$ is a disjoint sum of finite (topological) coverings of $U \cap Q$ which, in suitable local coordinates at $y_{0}$, are of the type considered in (10.5). 'This we proceed to show, using the control over ramification in codimension I provided by $\S 9$.

Fix $y_{0} \in Q_{\text {at }}$, and let $\mathrm{T}$ be the corresponding stablc partition of $\mathrm{S}$. Fix $a, b, c$ in distinct cosets. One can identify a neighborhood of $y_{0}$ in $Q_{8 t}$ with a subspace in $P^{S}$ whose clements take prescribed values on $a, b, c$. We arrange these values so that $\infty \notin y_{0}(\mathrm{~S})$. Near $y_{0}$, we then have the following system of local coordinates, depending on the choice of a representative $d(\mathrm{G})$ in each coset $\mathrm{G}$ :

a) the $y(d(\mathrm{C}))-y_{0}(d(\mathrm{G}))$, for $\mathrm{C}$ not the coset of $a, b$ or $c$;

b) for each coset $\mathrm{C}$, the $y(e)-y(d(\mathrm{G}))$ with $e \in \mathrm{C}-\{d(\mathrm{G})\}$.

In terms of thesc coordinates, the condition for $y$ near $y_{0}$ to be in $\mathbf{Q}$ is: $a$ ) for $\mathbf{G}$ a coset with three elements, $(y(e)-y(d(\mathbf{C})))_{e \in \mathrm{C}-\{d(\mathrm{C})\}} \in \mathbf{C}^{2}$ is not on any of the lines $\left.z_{1}=0, \quad z_{2}=0, z_{1}=z_{2} ; b\right)$ for $\mathrm{C}$ a coset with two elements, $y(e)-y(d(\mathrm{C})) \neq 0$ 
( $e \in \mathrm{C}, e \neq d(\mathrm{G}))$. We recall that $\mathrm{C}$ has at most three elements (6. I0. I), and that if $\mathrm{C}$ has three elements $x, y, z$, the sum of the reciprocal of the integers $\left(1-\mu_{z}-\mu_{y}\right)^{-1}$, $\left(1-\mu_{y}-\mu_{s}\right)^{-1},\left(1-\mu_{z}-\mu_{x}\right)^{-1}$ is $>1(6.10)$.

For $U$ a suitable open neighborhood of $y$ in $Q_{s t}$, we can hence apply (10.5) to the covering of $U \cap Q$ induced by $\widetilde{Q}$. We find that this covering breaks up into a disjoint sum of finite covering of the type required.

Lemma (10.7). - If condition INT is satisfied, the map $\widetilde{w}_{\mu}: \widetilde{Q}_{\mathrm{at}} \rightarrow \mathrm{B}^{+}(\alpha)_{o}$ is etale.

The map $\widetilde{w}_{\mu}$ is holomorphic on $\widetilde{Q}$ by (3.5) and continuous on $\widetilde{Q}_{\text {Bt }}$ by (8.7). It is hence holomorphic on $\widetilde{Q}_{a t}$. By (6.9), it has an injective differential on the strata of a suitable analytic stratification. The fibers of $\widetilde{w}_{\mu}$ hence have no component of dimension $>0$ and $\widetilde{w}_{\mu}$ is locally finite-to-one.

We know from $\S 9$ that $\widetilde{w}_{\mu}$ is etale outside of a closed analytic subset of $\widetilde{Q}_{\text {at }}$ of complex codimension $>2$. By the purity of the branch locus theorem (the fact that the branch locus is always purely of codimension one), it follows that $\widetilde{w}_{\mu}$ is etale everywhere.

(ro.8) Let us now assume, in addition to INT, that $Q_{\mathrm{At}}=Q_{\text {sat }}$, i.e. that for no $S(I) \subset S$ is $\sum_{s \in \mathbb{B}(1)} \mu_{\mathbf{s}}=\mathrm{I}$. Choose a metric on $Q_{\mathbf{t a}}$. We provide $\widetilde{Q}_{\mathbf{s t}}$ with the metric for which $d(x, y)$ is the infimum of the lengths of paths from $x$ to $y$, the length being measured by its projection into $Q_{\text {tt }}$. What we need is a metric invariant by the action of $\pi_{1}(Q, 0)$; any such metric will do. The space $\widetilde{Q}_{s t}$ is locally compact, and the projection to $Q_{\text {at }}$ is open. Since $Q_{\text {at }}$ is assumed to be compact, there is a compact $K \subset \widetilde{Q}_{\text {ot }}$ mapping onto $Q_{\mathrm{st}}$. Since $\widetilde{w}_{\mu}$ is etale, there are numbers $r, \mathrm{R}>0$ such that for $k \in \mathrm{K}$, the restriction of $\widetilde{w}_{\mu}$ to the ball $\mathrm{B}_{r}(k)$ of radius $r$ around $k$ in $\widetilde{Q}_{s t}$ is an embedding, and (10.8.1) $\widetilde{w}_{\mu}\left(\mathrm{B}_{r}(k)\right) \supset \mathrm{B}_{\mathbf{R}}\left(\widetilde{w}_{\mu}(k)\right)$.

The $\pi_{1}(Q, o)$ translates of $\mathrm{K}$ cover $\widetilde{Q}_{\mathrm{st}}$. The map $\widetilde{w}_{\mu}$ being equivariant, and the action on the ball $\mathrm{B}^{+}(\alpha)_{0}$ being via isometries, (10.8.I) holds for any $k \in \widetilde{\mathrm{Q}}_{\text {ot }}$. The map $\widetilde{w}_{\mu}$ is hence a covering map. The ball being simply connected

$$
\widetilde{w}_{\mu}: \widetilde{Q}_{\mathrm{ot}} \cong \mathrm{B}^{+}(\alpha)_{o}
$$

is an isomorphism. This concludes the proof of (3.II) in the cocompact case $\left(Q_{\text {et }}=Q_{\text {st }}\right)$, following the strategy outlined there.

(ro.9) Our second proof will bypass lemma (10.6). The purity of the branch locus theorem can be deduced from the fact that, if $\mathrm{Y}$ is a complex submanifold of a complex manifold $\mathrm{X}$ of complex codimension $\geq 2$, and $\mathrm{B}$ a small ball around $y \in \mathrm{Y}$, then $\mathrm{B}-\mathrm{Y}$ is simply connected. It is the latter fact which we will use directly to prove $(10.7)$.

The reader who merely wants to get the idea of how we complete the proof when $Q_{\text {ot }} \neq Q_{\text {st }}$ can skip to Proposition (10.18. I). 
(10.10) Our second proof of (IO.I.I) comes after proving that $\widetilde{w}_{\mu}$ is etale on $\widetilde{Q}_{88 t}$; that in turn is proved in stages.

Set $\mathbf{N}=$ card $\mathrm{S}$. For $i$ between o and $\mathrm{N}-3$, set

$$
Q_{i}=\amalg Q_{T}
$$

where $T$ runs through all stable partitions of cardinality $\geqslant$ card $S-i$. Then $Q_{0}=Q$, $Q_{N-3}=Q_{s t}$. Set $Q_{N-2}=Q_{s s t}$. Let $\widetilde{Q}_{i}$ denote the completion of $\widetilde{Q} \rightarrow Q$ over $Q_{i}(i=-1, \ldots, N-2)$. We shall prove inductively that $\widetilde{w}_{\mu}$ is etale on $Q_{i}$.

Proposition (10. 17) below will be used repeatedly to show at each stage that as one adds on a submanifold, the extended map $\widetilde{w}_{\mu}$ remains etale. We lead up to it via some general topological remarks, especially Proposition (Io.I5. I) and Corollary (10.15.4).

Proposition (ro.II). - Let $\varphi: \mathrm{X} \rightarrow \mathrm{Y}$ be a continuous map with $\mathrm{Y}$ locally connected and X Hausdorff. Assume that each $y \in \mathrm{Y}$ admits a neighborhood V such that each $x \in \varphi^{-1}(\mathrm{~V})$ has an open neighborhood $\mathrm{U}$ with $\varphi(\mathrm{U}) \supset \mathrm{V}$ such that $\varphi$ induces a homeomorphism from $\mathrm{U}$ to $\varphi(\mathrm{U})$. Then $\varphi$ is a covering map.

We first prove the

Lemma (ro.II. I). - Let $\varphi: \mathrm{X} \rightarrow \mathrm{Y}$ be a continuous map with $\mathrm{Y}$ connected and $\mathrm{X}$ Hausdorff. Let $\mathrm{X}_{1}$ and $\mathrm{X}_{2}$ be open in $\mathrm{X}$ and such that $\varphi$ induces homeomorphisms from $\mathrm{X}_{i}$ to $\mathrm{Y}$, $i=1,2$. Then $\mathrm{X}_{1}=\mathrm{X}_{2}$ or $\mathrm{X}_{1} \cap \mathrm{X}_{2}=\varnothing$.

Let $s_{i}: \mathrm{Y} \rightarrow \mathrm{X}$ be the inverse of $\varphi \mid \mathrm{X}_{i}$. The set of $y \in \mathrm{Y}$ with $s_{1}(y)=s_{2}(y)$ is closed. It is also open, being the image of $\mathrm{X}_{1} \cap \mathrm{X}_{2}$ by $\varphi: \mathrm{X}_{1} \sim \mathrm{Y}$. It is hence the whole of $\mathrm{Y}$ or empty; i.e. $\mathrm{X}_{1}=\mathrm{X}_{2}$ or $\mathrm{X}_{1} \cap \mathrm{X}_{2}=\emptyset$.

Proof of (10.11). - For VCY, let $\varphi_{\mathrm{V}}$ be the map induced by $\varphi$ from $\varphi^{-1}(\mathrm{~V})$ to $\mathrm{V}$. We have to show that each $y \in \mathrm{Y}$ has a neighborhood $\mathrm{V}$ such that

$$
\left(\varphi^{-1}(\mathrm{~V}), \varphi_{\mathrm{V}}\right) \simeq\left(\mathrm{V} \times \mathrm{D}, \mathrm{pr}_{1}\right)
$$

with $\mathrm{D}$ discretc. Take $\mathrm{V}$ as in (10.1 I), open and connected. Replacing the $\mathrm{U}$ of (Io.I I) by $U \cap \varphi^{-1}(V)$, we find that each $x \in \varphi^{-1} \mathrm{~V}$ is contained in an open set $U$ such that $\varphi$ induces a homeomorphism from $U$ to $V$. By (IO.II.I) applied to $\varphi^{-1}(V) \rightarrow V$, they form a partition of $\varphi^{-1}(V)$ and the claim follows.

Proposition (10.12). - Let $\varphi:(\mathrm{X}, o) \rightarrow(\mathrm{Y}, p)$ be a continuous map of metric spaces, with $\mathrm{Y}-\{\boldsymbol{p}\}$ locally connected. We write $d$ for the metric on both $\mathrm{X}$ and $\mathrm{Y}$. Assume that there exists a neighborhood $\mathrm{U}$ of o such that for each neighborhood $\mathrm{V}$ of 0 , one has

a) there exists $\varepsilon>0$ such that for all $x \in \mathrm{U}-\mathrm{V}$, the open ball $\mathrm{B}_{\varepsilon}(x)$ maps homeomorphically onto an open set of $\mathrm{Y}$;

b) for all $\varepsilon>0$ there is $\eta>0$ such that for each $x \in U-V, \varphi B_{\varepsilon}(x) \supset B_{\eta} \varphi(x)$.

Then, there exist open neighborhoods $\mathrm{X}^{\prime}$ of 0 and $\mathrm{Y}^{\prime}$ of $p$ such that $\varphi: \mathrm{X}^{\prime}-\varphi^{-1}(p) \rightarrow \mathrm{Y}^{\prime}-p$ is a covering map. 
Roughly speaking, a) b) mean that on $U-\{0\}, \varphi$ is a local homeomorphism with some uniformity. The uniformity is allowed to get worse for $x \rightarrow 0$. It is not assumed that $o$ is isolated in $\varphi^{-1}(p)$. Under additional assumptions, this will be a consequence; see (10.13).

Proof. - Choose U as in (1o. I2). Select a neighborhood V of 0 and $\varepsilon(1)>0$ such that $\mathrm{B}_{\varepsilon(1)}(\mathrm{V}):=\{x \in \mathrm{X} \mid \exists v \in \mathrm{V} d(x, v)<\varepsilon(\mathrm{I})\}$ is contained in $\mathrm{U}$. If $\mathrm{B}_{2 \varepsilon}(0) \subset \mathrm{U}$, one can take $\varepsilon(\mathrm{I})=\varepsilon$ and $\mathrm{V}=\mathrm{B}_{\varepsilon}(0)$. By a) b) there is $\varepsilon$ and $\eta$ such that

${ }^{*}$ ) for all $x \in \mathrm{U}-\mathrm{V}, \varphi$ induces a homeomorphism of $\mathrm{B}_{\varepsilon}(x)$ with an open set of $\mathrm{Y}$ containing $\mathrm{B}_{2 \eta}(\varphi(x))$.

Set $\mathrm{Y}^{\prime}=\mathrm{B}_{\eta}(p)$ and take $\mathrm{X}^{\prime}$ to be the set of all $x$ with $\varphi(x) \in \mathrm{Y}^{\prime}$ such that either

(i) $x \in \mathrm{V}$; or

(ii) there is $x_{1} \in \mathrm{U}-\mathrm{V}$ with $d\left(x, x_{1}\right)<\varepsilon$, and $\varphi\left(x_{1}\right) \in \mathrm{Y}^{\prime}$.

In case (ii), $x \in \mathrm{B}_{\mathbf{z}}\left(x_{1}\right) \cap \varphi^{-1}\left(\mathrm{Y}^{\prime}\right) \subset \mathrm{X}^{\prime}$ and $\varphi$ induces a homeomorphism of $\mathrm{B}_{\varepsilon}\left(x_{1}\right) \cap \varphi^{-1}\left(\mathrm{Y}^{\prime}\right)$ with $\mathrm{Y}^{\prime}$. This results from $\left(^{*}\right)$.

Fix $y \in \mathrm{Y}^{\prime}-p$ and let $\mathrm{W}$ be a neighborhood of $y$ in $\mathrm{Y}^{\prime}-\{p\}$, disjoint from a neighborhood of $p$. Then $\varphi^{-1} \mathrm{~W}$ is disjoint from a neighborhood of $o$ and by $a$ ) $b$ ) for $\varepsilon(2)$ small enough there is $\eta(2)$ such that

(*) for all $x \in \mathrm{U}$ with $\varphi(x) \in \mathrm{W}$, $\varphi$ induces a homeomorphism from $\mathrm{B}_{\boldsymbol{z}(2)}(x)$ to an open subset of $\mathrm{Y}$ containing $\mathrm{B}_{2 n(2)}(\varphi(x))$.

We may and shall assume that $\varepsilon(2)<\varepsilon(1)$ and that $B_{n(2)}(y) \subset W$. Proposition (I0.I2) now follows from (IO.II) applied to $\mathrm{X}^{\prime}-\varphi^{-1}(p) \rightarrow \mathrm{Y}-\{p\}$ and from the

Lemma (10.12.1). - Each $x$ in $\mathrm{X}^{\prime} \cap \varphi^{-1}\left(\mathrm{~B}_{n(2)}(y)\right)$ has an open neighborhood $\mathrm{B}^{\prime}$ in $\mathrm{X}^{\prime}$ which maps homeomorphically onto an open subset of $\mathrm{Y}$ containing $\mathrm{B}_{\eta(2)}(y)$.

As $x \in \mathrm{X}^{\prime}$, one of the conditions (i) (ii) holds. In the first case $(x \in \mathrm{V})$, one has $\mathrm{B}_{\varepsilon(2)}(x) \subset \mathrm{U}$, and we claim that $\mathrm{B}_{\varepsilon(2)}(x) \cap \varphi^{-1}\left(\mathrm{Y}^{\prime}\right) \subset \mathrm{X}^{\prime}$. For if $x^{\prime} \in \mathrm{B}_{\varepsilon(2)}(x)$ and $\varphi\left(x^{\prime}\right) \in \mathrm{Y}^{\prime}$, then either $x^{\prime} \in \mathrm{X}$, by virtue of (i) or $x^{\prime} \in \mathrm{U}-\mathrm{V}$ and $x^{\prime} \in \mathrm{X}^{\prime}$ by virtue of (i) with $x_{1}=x^{\prime}$. In the first case, $B^{\prime}=B_{\varepsilon(2)}(x) \cap \varphi^{-1}\left(Y^{\prime}\right)$ and one uses (*). In the second case, take $\mathrm{B}^{\prime}=\mathrm{B}_{\varepsilon}\left(x_{1}\right) \cap \varphi^{-1}\left(\mathrm{Y}^{\prime}\right)$ and use $\left(^{*}\right)$.

The assumptions of ( 10. I2) remain valid if we replace $X$ and $Y$ by open neighborhoods of $o$ and $p$. The $\mathrm{X}^{\prime}$ and $\mathrm{Y}^{\prime}$ of (10.12) can hence be chosen arbitrarily small. From this, we will deduce the

Corollary (10.12.2). - The point o has a fundamental system of open neighborhoods $\mathrm{X}^{\prime}$ such that for $\mathrm{Y}^{\prime}$ a suitable open neighborhood of $p, \mathrm{X}^{\prime}-\varphi^{-1}(p)$ is open and closed in $\varphi^{-1}\left(\mathrm{Y}^{\prime}-\{p\}\right)$ and is a covering of $\mathrm{Y}^{\prime}-\{p\}$. 
First take $\mathrm{X}_{0}^{\prime}, \mathrm{Y}_{0}^{\prime}$ as in (Io.I2), and $\mathrm{W}$ a neighborhood of $o$ such that $\mathrm{W}^{-} \subset \mathrm{X}_{0}^{\prime}$. If $\mathrm{X}^{\prime}, \mathrm{Y}^{\prime}$ are as in (10.12), with $\mathrm{X}^{\prime} \subset \mathrm{W}, \mathrm{Y}^{\prime} \subset \mathrm{Y}_{0}^{\prime}$ and if $\mathrm{X}_{1}^{\prime}:=\mathrm{X}_{0}^{\prime} \cap \varphi^{-1} \mathrm{Y}^{\prime}$, then both $\mathrm{X}_{1}^{\prime}-\varphi^{-1}(p)$ and $\mathrm{X}^{\prime}-\varphi^{-1}(p)\left(\subset \mathrm{X}_{1}^{\prime}-\varphi^{-1}(p)\right)$ are coverings of $\mathrm{Y}^{\prime}-\{p\}$; hence $\mathrm{X}^{\prime}-\varphi^{-1}(p)$ is open and closed in $\mathrm{X}_{1}^{\prime}-\varphi^{-1}(p)$. As $\left(\mathrm{X}^{\prime}-\varphi^{-1}(p)\right)^{-} \subset \mathrm{W}^{-} \subset \mathrm{X}_{0}^{\prime}$, $\mathrm{X}^{\prime}-\varphi^{-1}(p)$ is also open and closed in $\varphi^{-1}\left(\mathrm{Y}^{\prime}-\{p\}\right)$.

Corollary (10.12.3). - If 0 is not isolated in $\mathrm{X}$, and if $p$ has a fundamental system of neighborhoods $\mathrm{V}$ such that $\mathrm{V}-p$ is connected (in particular, non empty), then $\varphi$ is open at 0.

If $o$ is not isolated, $\mathrm{X}^{\prime}$ cannot be contained in $\varphi^{-1}(p)$; for applying (10.12) a) to $x$ in $\mathrm{X}^{\prime}$ near $o$ would contradict $\varphi\left(\mathrm{X}^{\prime}\right) \subset\{p\}$. It follows that $\varphi\left(\mathrm{X}^{\prime}-\varphi^{-1}(p)\right)=\mathrm{Y}^{\prime}-\{p\}$ and hence that $\varphi\left(X^{\prime}\right)=Y^{\prime}$. Openness follows.

Proposition (10.13). - In addition to the hypothesis of (10.12), assume that $\mathrm{Y}-\{p\}$ is locally simply connected and that

c) p has a fundamental system of open neighborhoods $\mathscr{V}$, such that each $\mathrm{V}-\{p\}(\mathrm{V}$ in $\mathscr{V})$ is connected and that for $\mathrm{V} \subset \mathrm{V}^{\prime}$, both in $\mathscr{V}, \pi_{1}(\mathrm{~V}-\{p\}) \underset{\sim}{\rightarrow} \pi_{1}\left(\mathrm{~V}^{\prime}-\{p\}\right)$.

d) o has a fundamental system of open neighborhoods $\mathrm{N}$ with $\mathrm{N}-\{0\}$ connected (nonempty).

Then, one can find $\mathrm{X}^{\prime}$ and $\mathrm{Y}^{\prime}$ as in (10.12) such that $\varphi^{\prime}: \mathrm{X}^{\prime} \rightarrow \mathrm{Y}^{\prime}$ has the following additional properties: $\mathrm{Y}^{\prime}$ is in $\mathscr{V}, \varphi^{\prime-1}(p)=\{0\}, \mathrm{X}^{\prime}-\{0\}$ is connected, and $\mathrm{X}^{\prime}$ is the completion of $\mathrm{X}^{\prime}-\{0\}$ above $\mathrm{Y}^{\prime}$.

Proof. of (10.13). - Shrinking $\mathrm{X}$ and $\mathrm{Y}$, we may and do assume that $\mathrm{Y}$ is in $\mathscr{V}$, that $\varphi: X-\varphi^{-1}(p) \rightarrow Y-\{p\}$ is a covering map, and that $\varphi$ is a local homeomorphism at each point $x \neq 0$. Let $\mathrm{N}$ be an open neighborhood of $o$ such that $\mathrm{N}-\{0\}$ is connected.

Lemma (ro.r3.I). $-\mathrm{N}-\varphi^{-1}(p)$ is connected.

Let $A \subset \mathrm{N}-\varphi^{-1}(p)$ be open and closed. Each $x \in \varphi^{-1}(p)-\{0\}$ has a neighborhood $W \subset N$ with $W-\{x\} \stackrel{\sim}{\rightarrow} \varphi(W)-\{p\}$ connected. For such a $W$, $\mathrm{W}-\{x\}=\mathrm{W}-\varphi^{-1}(p)$ lies either in $\mathrm{A}$ or in its complement. It follows that the closure of $\mathrm{A}$ in $\mathrm{N}-\{0\}$ is again open and close $\mathrm{J}$ and, by hypothesis, is empty or the whole of $\mathrm{N}-\{0\}$. If it is empty (resp. the whole of $\mathrm{N}-\{0\}$ ), $\mathrm{A}$ is empty (resp. the whole of $\left.\mathbf{N}-\varphi^{-1}(p)\right)$.

Since $\mathrm{Y}-\{p\}$ is locally connected, so is the covering space $\mathrm{X}-\varphi^{-1}(p)$. Each connected component of $\mathrm{X}-\varphi^{-1}(p)$ is open and closed in $\mathrm{X}-\varphi^{-1}(p)$ and is a covering of $\mathrm{Y}-\{p\}$. Let $\mathrm{N}_{1}$ be the component of $\mathrm{N}$ containing $\mathrm{N}-\varphi^{-1}(p)$.

Lemma (ro.r3.2). $-\mathrm{N}_{1} \cap \varphi^{-1}(p)=\{0\}$.

Any $x \neq 0$ in $\varphi^{-1}(p)$ has an open neighborhood $W^{\prime}$ such that $\varphi$ induces a homeomorpicism of $W^{\prime}$ with an open neighborhood of $p$ in $Y$. Take $V$ in $\mathscr{V}$ with $V \subset \varphi\left(W^{\prime}\right)$

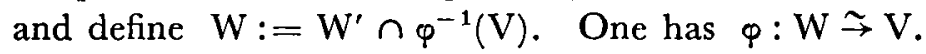


The assumption $\pi_{1}(V-\{p\}) \stackrel{\sim}{\rightarrow} \pi_{1}(Y-\{p\})$ implies that for any covering map $\psi: Z \rightarrow Y-\{p\}$, one has $\pi_{0}\left(\psi^{-1}(V-\{p\})\right) \stackrel{\sim}{\rightarrow} \pi_{0}(Z)$. In particular, as $N_{1}$ is connected, so is $N_{1} \cap \varphi^{-1}(V)$. Both $N_{1} \cap \varphi^{-1}(V)$ and $W-\{x\}$ are connected coverings of $\mathrm{V}-\{p\}$, contained in the covering $\varphi^{-1}(\mathrm{~V}-\{\boldsymbol{p}\})$. They are not equal: $x$ is the only point of $\varphi^{-1}(p)$ in $\mathrm{W}^{-}$, while $o \in \mathrm{N}_{1}^{-}$. They are hence disjoint and $x \notin \mathrm{N}_{1}$. Lcmma (1o. 13.2 ) is proved.

We take $\mathrm{Y}^{\prime}=\mathrm{Y}$ and $\mathrm{X}^{\prime}=\mathrm{N}_{1}^{-}$. It follows from (10.13.2) that $\mathrm{X}^{\prime}$ is open and closed in $\mathrm{X}$ and that $\varphi^{-1}(p)=\{0\}$. By construction, $\mathrm{X}^{\prime}-\varphi^{-1}(p)=\mathrm{X}^{\prime}-\{0\}$ is connected. For each $\mathrm{V}$ in $\mathscr{V}, \pi_{1}(\mathrm{~V}-\{p\}) \stackrel{\sim}{\rightarrow} \pi_{1}\left(\mathrm{Y}^{\prime}-\{p\}\right)$ implies as above that $\varphi^{-1}(\mathrm{~V}-\{p\})$ is connected. It then follows from (Io.12.2) that the $\varphi^{-1}(\mathrm{~V}) \cap \mathrm{X}^{\prime}$ $(\mathrm{V} \in \mathscr{V})$ form a fundamental system of neighborhoods of $o$ in $\mathrm{X}^{\prime}$. Consequently $\mathrm{X}^{\prime}$ is the completion of $\mathrm{X}^{\prime}-\{0\}$ over $\mathrm{Y}^{\prime}$.

Corollary (10.13.3). - If, in addition to the assumptions of (10.12) and (10.13): the $\mathrm{V}-\{\boldsymbol{p}\}$ for $\mathrm{V}$ in $\mathscr{V}$ are simply connected, then $\varphi$ is a local homeomorphism at 0.

Indeed, with the notation of (10.13), $\mathrm{Y}^{\prime}-\{p\}$ is simply connected; hence $\varphi: \mathrm{X}^{\prime}-\{0\} \rightarrow \mathrm{Y}^{\prime}-\{p\}$ is a homeomorphism as well as $\varphi^{\prime}: \mathrm{X}^{\prime} \rightarrow \mathrm{Y}^{\prime}$.

(ro.14) We shall require a simpler variant of (10.12) in our extension of the map $\widetilde{w}_{\mu}$ to cusp points. In that situation, we shall be dealing with a continuous map $\varphi:(\mathrm{X}, 0) \rightarrow(\mathrm{Y}, p)$. Metrics are given only on $\mathrm{X}-\{0\}$ and $\mathrm{Y}-\{p\}$, and we assume

a) The $\varphi^{-1}(\mathrm{~V})$, for $\mathrm{V}$ a neighborhood of $p$ in $\mathrm{Y}$, form a fundamental system of neighborhoods of 0 . In particular, $\varphi^{-1}(p)$ is $\{0\}$.

b) There is a neighborhood $U$ of $o$ such that for any neighborhood $V \subset U$, the conditions $a$ ), b) of (10.12) are satisfied.

Fix a neighborhood $V_{1}$ of $p$ such that $\varphi^{-1}\left(V_{1}\right) \subset U$. For any neighborhood $V_{2}$ of $p$ and $x \in V_{1}-V_{2}$, the map $\varphi_{1}: \varphi^{-1}\left(V_{1}-V_{2}\right) \rightarrow V_{1}-V_{2}$ induces a homeomorphism of $\mathrm{B}_{\varepsilon}(x)$ with an open set containing $\mathrm{B}_{\eta}\left(\varphi_{1}(x)\right)$ for suitable $\varepsilon$ and $\eta$. The map $\varphi_{1}$ is hence a covering map. As this holds for any $V_{2}, \varphi: \varphi^{-1}\left(V_{1}-\{p\}\right) \rightarrow V_{1}-\{p\}$ is also a covering map.

If in acldition

c) $p$ has a fundamental system of neighborhoods $V$ such that $V-\{p\}$ is connected and simply connected,

d) o has a fundamental system of neighborhoods $\mathrm{N}$ such that $\mathrm{N}-\{0\}$ is connected, then an easy argument shows that $\varphi$ is a local homeomorphism at $o$.

(ro.r5) Actually, we require (I0.13.3) for the more general case where 0 and $p$ are replaced by strata. The analogue of (10.12) is:

Proposition (10.15.I). - Let $\varphi:\left(\mathrm{X}, \mathrm{X}_{1}, 0\right) \rightarrow\left(\mathrm{Y}, \mathrm{Y}_{1}, p\right)$ be a continuous map of metric spaces, with $\mathrm{Y}-\mathrm{Y}_{1}$ locally connected. Assume that there exists a neighborhood $\mathrm{U}$ of o such that 
for each neighborhood $\mathrm{V}$ of $\mathrm{X}_{1}$, the conditions a) b) of (10.12) hold. Assume further that $\mathrm{X}_{1}$ is locally compact and that the map $\varphi_{1}: \mathrm{X}_{1} \rightarrow \mathrm{Y}_{1}$ induced by $\varphi$ is a local homeomorphism at 0 . Then there are open neighborhoods $\mathrm{X}^{\prime}$ of $o$ and $\mathrm{Y}^{\prime}$ of $p$ such that $\varphi: \mathrm{X}^{\prime}-\varphi^{-1}\left(\mathrm{Y}_{1}\right) \rightarrow \mathrm{Y}^{\prime}-\mathrm{Y}_{1}$ is a covering map.

Proof. - F $1 \mathrm{x}$ U as above, open and small enough so that for some open set $\mathrm{R}$ disjoint from $U, K:=X_{1}-R$ is compact and so that $\varphi$ is injective on $K$. Note that if we take $\mathrm{R}=\mathrm{X}-\mathrm{U}^{-}$, then $\mathrm{K}=\mathrm{X}_{1} \cap \mathrm{U}^{-}$.

The subset $\varphi(\mathrm{K}-\mathrm{U})$ of $\mathrm{Y}_{1} \subset \mathrm{Y}$ is compact and does not contain $p$. Let $\mathrm{W}$ be an open neighborhood of $\varphi(\mathrm{K}-\mathrm{U})$ whose closure does not contain $p$. Then, $\varphi^{-1} \mathrm{~W}$ is an open neighborhood of $K-U$, and $\varphi^{-1}\left(W^{-}\right)$does not contain 0 .

Select a neighborhood $V$ of $X_{1}$, and $\varepsilon(I)>o$ such that

$$
\mathrm{B}_{\varepsilon(1)}\left((\mathrm{V} \cap \mathrm{U})-\varphi^{-1}\left(\mathrm{~W}^{-}\right)\right) \subset \mathrm{U} \text {; }
$$

this can be done as follows. Since $K-\varphi^{-1} W$ is compact and in $U$, we choose $\varepsilon(I)>0$ so that $B_{2 \varepsilon(1)}\left(K-\varphi^{-1} W\right) \subset U$. One takes $V=B_{\varepsilon(1)}\left(K-\varphi^{-1} W\right) \cup \varphi^{-1} W \cup R$. Then $(V \cap U)-\varphi^{-1} W \subset B_{\varepsilon(1)}\left(K-\varphi^{-1} W\right)$ and $B_{\varepsilon(1)}\left((V \cap U)-\varphi^{-1}\left(W^{-}\right)\right) \subset U$ by choice of $\varepsilon(\mathbf{I})$

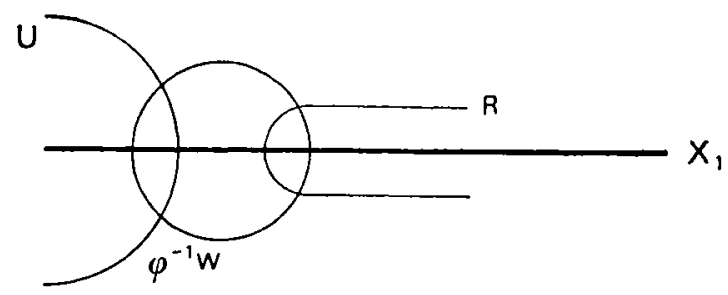

By $a), b)$, there are $\varepsilon$ and $\eta$ such that

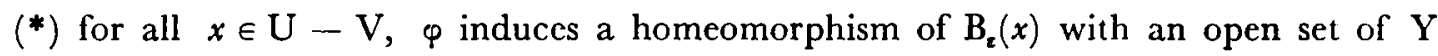
containing $\mathrm{B}_{2 n}(\varphi(x))$.

We may and do choose $\eta$ small enough so that $B_{\eta}(p)$ is disjoint from $W^{-}$. Set $\mathrm{Y}^{\prime}=\mathrm{B}_{n}(p)$ and takc $\mathrm{X}^{\prime}$ to be the set of all $x$ with $\varphi(x) \in \mathrm{Y}^{\prime}$ (hence $x \notin \varphi^{-1}\left(\mathrm{~W}^{-}\right)$) such that cither $x \in \mathrm{U} \cap \mathrm{V}$, or there is $x_{1} \in \mathrm{U}-\mathrm{V}$ with $d\left(x, x_{1}\right)<\varepsilon$ and $\varphi\left(x_{1}\right) \in \mathrm{Y}^{\prime}$.

Any $y \in \mathrm{Y}-\mathrm{Y}_{1}$ has a neighborhood $\mathrm{W}_{y}$ disjoint from a neighborhood of $\mathrm{Y}_{1}$; hence, as in (I0.I2), for $\varepsilon(2)$ small enough there is $\eta(2)$ such that

(**) for all $x \in \mathrm{U}$ with $\varphi(x) \in \mathrm{W}_{y}, \varphi$ induces a homeomorphism from $\mathrm{B}_{\varepsilon(2)}(x)$ to an open set of $\mathrm{Y}$ containing $\mathrm{B}_{2 \eta(2)}(\varphi(x))$.

One can assume that $\varepsilon(2)<\varepsilon(1)$ and $B_{\eta(2)}(y) \subset W$, and one completes the proof just as in (10.12), using that $\varphi(x) \in \mathrm{Y}^{\prime}$ implies $x \notin \varphi^{-1} \mathrm{~W}^{-}$.

(10.15.2) As in (10.12.2), $\mathrm{X}^{\prime}$ can be taken as small as we please. If $o$ is not an interior point of $\mathrm{X}_{1}$, and if $p$ has a fundamental system of neighborhoods $\mathrm{V}$ with $\mathrm{V}-\mathrm{Y}_{1}$ connected, $\varphi$ is open at $o$. The proof is as in (10.12.3). 
Proposition (10.15.3). - Assume that $\mathrm{Y}-\mathrm{Y}_{1}$ is locally simply connected and that

c) $p$ has an open neighborhood $V_{0}$ with $V_{0}-Y_{1}$ connected, and each $p^{\prime} \in Y_{1}$ close enough to $p$ has a fundamental system of neighborhoods $\mathrm{V} \subset \mathrm{V}_{0}$ with $\mathrm{V}-\mathrm{Y}_{1}$ connected and

$$
\pi_{1}\left(\mathrm{~V}-\mathrm{Y}_{1}\right) \stackrel{\sim}{\rightarrow} \pi_{1}\left(\mathrm{~V}_{0}-\mathrm{Y}_{1}\right) \text {. }
$$

d) Each $o^{\prime} \in \mathrm{X}_{1}$ close enough to o has a fundamental system of neighborhoods $\mathrm{N}$ with $\mathrm{N}-\mathrm{X}_{1}$ connected.

Then one can find $\mathrm{X}^{\prime}$ and $\mathrm{Y}^{\prime}$ as in (I0. I5. I) such that $\varphi^{\prime}: \mathrm{X}^{\prime} \rightarrow \mathrm{Y}^{\prime}$ has the following additional properties: c) holds with $\mathrm{V}_{0}=\mathrm{Y}^{\prime}$ for all $p^{\prime} \in \mathrm{Y}_{1} \cap \mathrm{V}_{0}, \mathrm{X}^{\prime}-\mathrm{X}_{1}$ is connected, $\mathrm{X}_{1}^{\prime}:=\mathrm{X}_{1} \cap \mathrm{X}^{\prime}$ is the inverse image of $\mathrm{Y}_{1}^{\prime}:=\mathrm{Y}_{1} \cap \mathrm{Y}^{\prime}$, $\varphi^{\prime}$ induces a homeomorphism from $\mathrm{X}_{1}^{\prime}$ to $\mathrm{Y}_{1}^{\prime}$, and $\mathrm{X}^{\prime}$ is the completion of $\mathrm{X}^{\prime}-\mathrm{X}_{1}^{\prime}$ above $\mathrm{Y}^{\prime}$.

Proof. - Shrinking $\mathrm{X}$ and $\mathrm{Y}$, we may and do assume that c) holds for $\mathrm{V}_{0}=\mathrm{Y}$ and for all $p^{\prime} \in Y_{1} \cap V_{0}$, that $\varphi_{1}: X_{1} \rightarrow Y_{1}$ is a homeomorphism, that $d$ ) holds for all $o^{\prime} \in \mathrm{X}_{1}$, that $\varphi: \mathrm{X}-\varphi^{-1}\left(\mathrm{Y}_{1}\right) \rightarrow \mathrm{Y}_{1}$ is a covering map, and that $\varphi$ is a local homeomorphism at each point $x \notin \mathrm{X}_{1}$. Let $\mathrm{N}$ be an open neighborhood of 0 with $\mathrm{N} \cdots \mathrm{X}_{1}$ connected. $A s$ in (10.13.I), one checks that $N-\varphi^{-1} Y_{1}$ is connected. Let $N_{1}$ be the connected component of $X-\varphi^{-1} Y_{1}$ containing $N-\varphi^{-1} Y_{1}$. It is a covering of $Y-Y_{1}$.

If we shrink $Y$ again without changing the $\pi_{1}\left(Y-Y_{1}\right)$, and replace $X, N, N_{1}$ by their traces on the pull-back of the new $Y$, we get $N-X_{1} \subset N_{1}$ with $N_{1}$ connected and $\mathrm{N} \supset \mathrm{X}_{1}$.

As in (10.13.2), one sees that $N_{1}^{-} \cap \varphi^{-1} Y_{1}=X_{1}$. One takes $Y^{\prime}=Y, X^{\prime}=N_{1}^{-}$ and $\varphi^{\prime}: X^{\prime} \rightarrow Y^{\prime}$ induced by $\varphi$. The set $X^{\prime}$ is open and closed in the (shrunken) $X$ and $\varphi^{\prime-1}\left(Y_{1}^{\prime}\right)=X_{1}^{\prime}$. All the listed properties of $\varphi^{\prime}$ are clear from the construction except for $\mathrm{X}^{\prime}$ being the completion of $\mathrm{X}^{\prime}-\mathrm{X}_{1}^{\prime}$ over $\mathrm{Y}^{\prime \prime}$. For $p \in \mathrm{Y}_{1}^{\prime}$, $p^{\prime}=\varphi^{\prime}\left(o^{\prime}\right)$, and $V$ an open neighborhood of $p^{\prime}$ with $\pi_{1}\left(V-Y_{1}^{\prime}\right) \stackrel{\sim}{\rightarrow} \pi_{1}\left(Y-Y_{1}^{\prime}\right)$, one has $\pi_{0}\left(\varphi^{\prime-1}\left(V-Y_{1}^{\prime}\right)\right) \stackrel{\sim}{\rightarrow} \pi_{0}\left(X^{\prime}-X_{1}^{\prime}\right)$, i.e. $\varphi^{\prime-1}\left(V-Y_{1}^{\prime}\right)$ is connected. The $\varphi^{\prime-1}(\mathrm{~V})$ for such $\mathrm{V}$ form a fundamental system of neighborhoods of $o^{\prime}$ and the assertion about completion follows.

In particular,

Corollary (10.15.4). - In addition to the hypothesis of (I0.I5.I) assume that $\mathrm{Y}-\mathrm{Y}_{1}$ is locally simply connected and that

$\left.c^{\prime}\right)$ Each $p^{\prime} \in \mathrm{Y}_{1}$ close to $p$ has a fundamental system of neighborhood $\mathrm{V}$ with $\mathrm{V}-\mathrm{Y}_{1}$ connected and simply connected.

d) Each $o^{\prime} \in \mathrm{X}_{1}$ close to o has a fundamental system of neighborhood $\mathrm{V}$ with $\mathrm{V}-\mathrm{X}_{1}$ connected.

Then $\varphi$ is a local homeomorphism at 0 .

(10.16) We will apply (10.15.4) iteratively to some stratified spaces. In the next proposition, "stratified space" means a Haussdorf topological space U, provided with a partition $\mathbf{S}$ into locally closed subsets, the strata, where 
a) each stratum is a manifold; b) the closure of any stratum $\mathrm{S}$ is the union of strata; $(\mathbf{U}, \mathbf{S})$ is conical in the following sense and locally constant along each stratum: each point $x$ of any stratum $\mathrm{S}$ has a neighborhood $\mathrm{V}$ isomorphic (with the induced partition) to the product of $\mathrm{V} \cap \mathrm{S}$ with the cone over $(\Sigma, \mathbf{S}(\Sigma))$, where $\Sigma$ is a topological space $\Sigma$, provided with a partition $\mathbf{S}(\Sigma)$. For simplicity, we assume in addition the existence of a metric $d$ for which $d(x, y)$ is the greatest lower bound of lengths of paths from $x$ to $y$. The case we will need is $\mathrm{U}=\mathrm{Q}_{\mathrm{st}}, \mathbf{S}=$ the partition of $\mathbf{Q}_{\mathrm{st}}$ by the $Q_{T}$ for $T$ varying over the stable partitions.

Proposition (10.16.1). - Consider a diagram

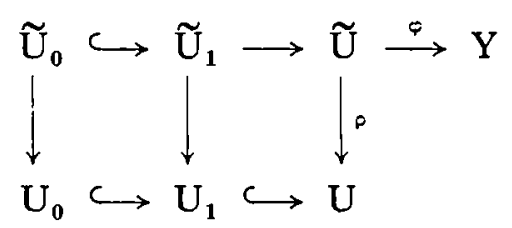

where

a) $\mathrm{U}$ is locally compact stratified space; $\mathrm{U}_{0}$ is a connected open and dense stratum, and $\mathrm{U}_{\mathbf{1}}-\mathrm{U}_{\mathbf{0}}$ is a union of strata; each point has a fundamental system of neighborhoods whose traces on $\mathrm{U}_{0}$ are connected.

b) $\widetilde{\mathrm{U}}_{0}$ is a covering space of $\mathrm{U}_{0}$, and $\tilde{\mathrm{U}}_{1}$ (resp. $\tilde{\mathrm{U}}$ ) is the completion of $\tilde{\mathrm{U}}_{0}$ over $\mathrm{U}_{1}$ (resp. $\mathrm{U}$ ).

c) The covering $\widetilde{U}_{0}$ of $U_{0}$ is Galois, with group $\Gamma$.

d) $\mathrm{Y}$ is a manifold, provided with a metric and an action of $\Gamma$ by isometries. The map $\varphi$ is equivariant.

e) $\varphi \mid \tilde{U}_{1}$ is a local homeomorphism.

f) For any stratum $\mathrm{S}, \widetilde{\mathrm{S}}:=\mathrm{\rho}^{-1}(\mathrm{~S})$ is a covering space of $\mathrm{S}$ because $\mathrm{U}_{0}$ is a stratum. We assume that for $\mathrm{S} \subset \mathrm{U}-\mathrm{U}_{1}$, each point of $\widetilde{\mathrm{S}}$ has a neighborhood $\mathrm{W}$ in $\widetilde{\mathrm{S}}$ such that $\varphi \mid \mathrm{W}$ is a tame embedding, with $\varphi(\mathrm{W})$ a subvariety of codimension $\geqslant 3$ in $\mathrm{Y}$.

Then, $\varphi$ is a local homeomorphism and the (local) decomposition groups of $\widetilde{\mathrm{U}} / \mathrm{U}$ are finite, i.e. $p$ is locally finite to one.

Proof. -.- Let $d$ be a $\Gamma$-invariant metric on $\widetilde{\mathrm{U}}$; for instance take $d(x, y)=$ the greatest lower bound of the lengths, computed in $\mathrm{U}$, of paths in $\tilde{\mathrm{U}}$ from $x$ to $y$.

Let $\mathrm{S}$ bc an open stratum of $\mathrm{U}-\mathrm{U}_{1}$. We first prove that $\varphi$ is a local homeomorphism at any point $o \in \rho^{-1}(S)$. For this, replacing $U$ by a suitable open neighborhood $\mathrm{V}$ of $\rho(0), \widetilde{U}$ by the connected component of $\rho^{-1}(V)$ containing o and $\Gamma$ by the stabilizer of $o$, we may and shall assume that $U-U_{1} \in S$, that $p$ induces an isomorphism $\widetilde{S} \rightarrow \mathrm{S}$ and that $\varphi$ embeds $\widetilde{\mathrm{S}}$ in $\mathrm{Y}$. Replacing $\mathrm{Y}$ by a $\Gamma$-stable open neighborhood of $\varphi(0)$, and $\tilde{U}$ by its inverse image, one may further assume that $\mathrm{Y}_{1}:=\varphi(\widetilde{\mathrm{S}})$ is a tame closed submanifold of $\mathrm{Y}$. Its codimension is $\geqslant 3$. We will apply (I0. 15.4) with $\mathrm{X}=\tilde{\mathrm{U}}, \mathrm{X}_{1}=\widetilde{\mathrm{S}}$ and $0, \mathrm{Y}, \mathrm{Y}_{1}, \rho$ as above. Local connectedness and simple connectedness of $\mathrm{Y}-\mathrm{Y}_{1}$ results from $\mathrm{Y}$ being a manifold. Condition $c^{\prime}$ ) 
of (10.15.4) results from $Y_{1}$ being a tame submanifold of codimension $\geqslant 3$. Condition d) of (10.15.4) results from assumption $a)$. By $e) \varphi \mid\left(X-X_{1}\right)$ is a local homeomorphism, and it remains to check the uniformity conditions of (I0.I2). As neighborhood of 0 , we choose the pull back $p^{-1}(K)$ of a compact neighborhood $K$ of $p(0) \in U$. By definition of the topology of $\tilde{U}$, any neighborhood of $X_{1}=\widetilde{S} \sim \mathcal{S}$ contains $p^{-1}(V)$, for $V$ an open neighborhood of $S$. The required uniformities will follow from the compactness of $\mathrm{K}-\mathrm{V}$. The function from $\mathrm{X}-\mathrm{X}_{1}$ to $\mathbf{R}^{+}: x \mapsto$ the greatest lower bound of the $\varepsilon$ such that $\varphi$ is an embedding on $B_{\varepsilon}(x)$, is $>0$, lower semi-continuous, and $\Gamma$-invariant; hence it is of the form $r_{p}$ with $r$ continuous. On $\mathrm{K}-\mathrm{V}, r$ stays away from $o$, and this gives (10.12a)). If $\varepsilon$ is such that for any $x \in p^{-1}(\mathrm{~K}-\mathrm{V}), \varphi \mid \mathrm{B}_{2 \varepsilon}(x)$ is an embedding, the function from $\rho^{-1}(\mathrm{~K}-\mathrm{V})$ to $\mathbf{R}^{-}: x \mapsto$ the greatest lower bound of the $\eta$ such that $\varphi\left(\mathrm{B}_{\varepsilon}(x)\right) \supset \mathrm{B}_{n}(\varphi(x))$ is $>\mathbf{o}$, lower semi-continuous and $\Gamma$-invariant, hence of the form $r_{\rho}$ and staying away from 0 . This gives (10.12b)).

We now prove that $\varphi: \tilde{U} \rightarrow Y$ is a local homeomorphism. We have just shown that $\varphi$ is a local homeomorphism on $\rho^{-1}\left(U_{1}^{\prime}\right)$, for $U_{1}^{\prime}$ the union of $U_{1}$ and of the open strata of $U-U_{1}$. The assumptions of the Proposition hence hold with $U_{1}$ (resp. $\widetilde{U}_{1}$ ) replaced by $U_{1}^{\prime}$ (resp. $\left.p^{-1} U_{1}^{\prime}\right)$, and one concludes it proof by induction on $\operatorname{dim}\left(U_{-}-U_{1}\right)$.

It remains to show that for any $x \in \tilde{\mathrm{U}}$, the stabilizer $\Delta \subset \Gamma$ of $x$ is finite. Fix a neighborhood $\mathrm{V}$ of $x$ such that $\varphi \mid \mathrm{V}$ is an open embedding, fix a $\Delta$-stable compact neighborhood $\mathrm{K}$ of $\varphi(x)$ in $\varphi(\mathrm{V})$, and define $\mathrm{V}_{1}=\mathrm{V} \cap \varphi^{-1} \mathrm{~K}$. It is a $\Delta$-invariant compact neighborhood of $x$. For $y \in V_{1} \cap \widetilde{U}_{0}, \Gamma y$ is closed and discrete. Therefore $\Delta y$, which is in $\Gamma y \cap V_{1}$, is discrete and compact, and hence finite. Thus $\Delta$ is finite.

We will use (I0.I6. I) to give an alternate proof of lemma (10.7), by passing the purity of the branch locus theorem (which was derived in the special case $Q_{\mathrm{st}}=Q_{\mathrm{st}}$ ).

Lemma (ro.17). - If condition (INT) is satisfied, the map $\widetilde{w}_{\mu}: \widetilde{\mathrm{Q}}_{\mathrm{Bt}} \rightarrow \mathrm{B}^{+}(\alpha)_{0}$ is a local homeomorphism.

Proof. - We apply (10.16.1) with $\mathrm{U}=\mathrm{Q}_{\mathrm{si}}$ stratified by the $Q_{\mathrm{T}}$ for $\mathrm{T}$ varying over the stable partitions, $U_{0}=Q, U_{1}=$ the union of $Q$ and of the (complex) codimension one strata, $\widetilde{\mathrm{U}}_{0}=\widetilde{\mathrm{Q}}$; hence $\widetilde{\mathrm{U}}=\widetilde{\mathrm{Q}}_{\mathrm{st}}$ and $\varphi=\widetilde{w}_{\mu}$. Conditions $a$ ) to $d$ ) are clear, $e)$ is (9.12) and $f)$ follows from (6.9) and (3.9).

(10. 18). - As shown in (8.7), the map $\widetilde{w}_{\mu}$ extends to a continuous map from $\widetilde{Q}_{\text {sst }}$ to $\widetilde{\mathrm{B}}^{+}:=\overline{\mathrm{B}}^{+}(\alpha)_{0}$, where $\overline{\mathrm{B}}^{+}$has the topology (5.4). Fix $\mathrm{J} \in \mathrm{Q}_{\text {cusp }}$ and $0 \in \widetilde{\mathrm{Q}}_{\mathrm{sst}}$ above J. Define $\rho:=\widetilde{w}_{\mu}(o)$.

I.et $\mathrm{V}$ be an open connected neighborhood of $\mathrm{J}$, and $\widetilde{\mathrm{V}}$ be the connected component containing o of its inverse image in $\widetilde{Q}_{\text {sst }}$. For $V$ small enough, one has: $o$ is the only point of $\widetilde{V}$ above $J$; the stabilizer $\Delta C \Gamma$ of a acts on $\widetilde{V}$, and $V=\widetilde{V} / \Delta$; if $\ell$ is a distance to $p$ function $(5 \cdot 3)$ on $\overline{\mathrm{B}}^{+}, \ell \widetilde{w}_{\mu}$ descends to a continuous function $f$ on $\mathrm{V}$, vanishing only at $\mathrm{J}$. As $\mathrm{V}$ is locally compact, it follows, for $\mathrm{V}$ small enough, that the open sets $\{v \mid f(v)<\varepsilon\}$ form a fundamental system of neighborhood of J. As a consequence, 
the $\tilde{w}_{\mu}^{-1}(\mathrm{~A})$, for $\mathrm{A}$ a neighborhood of $p$, form a fundamental system of neighborhood of $o$. We now apply (ro. I4) to prove

\section{Lemma (10.18.x). - If condition (INT) is satisfied, the map $\widetilde{w}_{\mu}: \widetilde{Q}_{\text {sat }} \rightarrow \overline{\mathrm{B}}^{+}$is a local homeomorphism.}

Proof. - It remains to prove it at a point o as above. We apply (10. I4) to $\widetilde{w}_{\mu}:(\tilde{\mathrm{V}}, o) \rightarrow\left(\overline{\mathrm{B}}^{1}, p\right)$. Condition $\left.a\right)$ has been proved. Condition $\left.c\right)$ and $\left.d\right)$ are clear. The map is a local homeomorphism outside of 0 . The required uniformity (10.14 $b$ )) follows as before from the local compactness of $\mathrm{V}$ and $\Delta$-equivariance.

Theorem (10.18.2). - Assume condition (INT). Then the map $\widetilde{w}_{\mu}: \mathbf{Q}_{\mathrm{sat}} \rightarrow \mathrm{B}^{+}$ restricts to a homeomorphism of $\widetilde{Q}_{\mathrm{at}}$ onto $\mathrm{B}^{+}$and maps $\widetilde{Q}_{\mathrm{Bst}}$ homeomorphically onto an open subset of $\overline{\mathrm{B}}^{+}$in the (5.4) topology.

Proof. - By Proposition (10.18.I), $\widetilde{w}_{\mu}$ is an etale map of $\widetilde{\mathrm{Q}}_{\text {stt }}$ into $\overline{\mathrm{B}}^{+}$. Let $\varphi$ denote the restriction of $\widetilde{w}_{\mu}$ to $\widetilde{Q}_{s t}$. It suffices to prove that $\varphi$ is an covering map of $\widetilde{Q}_{s t}$ onto $\mathrm{B}^{+}$.

For any $x \in \widetilde{Q}_{\text {at }}$, set

$f(x)=\sup \left\{r \mid \varphi\right.$ maps a neighborhood of $x$ homeomorphically onto $\left.\mathrm{B}_{r}(\varphi(x))\right\}$.

Then as in the proof of Lemma (I0.16. I), $f$ is continuous, $f(x)>0$ for $x \in \widetilde{Q}_{\text {st }}$ and $f(\gamma x)=f(x)$ for all $\gamma \in \pi_{1}(Q, o)$; that is, $f$ descends to a positive-valued continuous function on $Q_{B t}$.

Since $\widetilde{w}_{\mu}$ is etale at each point of $\widetilde{Q}_{\text {sst }}$ and $Q_{\text {ousp }}$ consists only of finitely many points, one can deduce from the (5.4) topology of $\bar{B}^{+}$the existence of an open neighborhood $W$ of $Q_{\text {cusp }}$ and an $\eta_{1}>0$ such that $f(x) \geq \eta_{1}$ for all $x \in \rho^{-1}(W)$. By $\S 4$, $Q_{\text {sot }}$ is compact. From the compactness of $Q_{\text {ot }}-W$ one deduces that $f$ has a nonzero lower bound on $\widetilde{Q}_{\text {at }}-p^{-1}(W)$. Consequently, $f$ has a non-zero lower bound $\eta$ on $\widetilde{\mathbf{Q}}_{\mathrm{st}}$. From this it follows that $\mathbf{B}_{n}\left(\varphi\left(\widetilde{\mathbf{Q}}_{\mathrm{st}}\right)\right)=\varphi\left(\widetilde{\mathbf{Q}}_{\mathrm{st}}\right)$, and therefore, $\varphi\left(\widetilde{\mathbf{Q}}_{\mathrm{st}}\right)=\mathbf{B}^{+}$. By (IO.II), $\varphi$ is a covering map. Inasmuch as $B^{+}$is simply connected, $\varphi$ is a homeomorphism.

From the fact that each point $y \in Q_{\text {ousp }}$ has a base of neighborhoods $\{\mathrm{V}\}$ with $\mathrm{V}-\mathrm{Y}$ connected, and the fact that $\widetilde{w}_{\mu}$ is a homeomorphism on $\widetilde{Q}_{\text {st }}$, it follows that $\widetilde{w}_{\mu}$ is injective on $\widetilde{Q}_{\text {ast }}$ and hence a homeomorphism onto its image, in the topology of $(5 \cdot 4)$.

(1o.19) Let $S$ be a finite set with at least 3 elements, $N=$ card $S, \mu=\left(\mu_{s}\right)_{, \in s}$ with $0<\mu_{\mathrm{s}}<\mathrm{I}$ and $\Sigma \mu_{\mathrm{a}}=2$. Set $\alpha_{\mathrm{a}}=\exp 2 \pi \sqrt{-\mathrm{I}} \mu_{\mathrm{s}}$ for each $s \in \mathrm{S}, \mathrm{P}$ the projective line over $\mathbf{C}, G=$ Aut $P, M$ the subset of injective elements in $P^{8}, Q=M / G$, $o \in Q$, and $\theta: \pi_{1}(Q, o) \rightarrow \Lambda u t B^{+}(\alpha)_{0}=\mathrm{PU}(\mathrm{r}, \mathrm{N}-2)$ the homomorphism of $\pi_{1}(\mathrm{Q}, 0)$ into the isometry group of the ball $\mathrm{B}^{+}(\alpha)_{0}$, defined in (3.10.2). Set $\Gamma=\theta\left(\pi_{1}(\mathrm{Q}, 0)\right)$, $\mathrm{B}^{+}=\mathrm{B}^{+}(\alpha)_{a}$. 
Theorem (10.19). - If $\mu$ satisfies condition (INT), then $\Gamma$ is a discrete subgroup of Aut $\mathrm{B}^{+}$ and discontinuous on $\mathrm{B}^{+}$.

Proof. - By Theorem (10.17.I), the action of $\Gamma$ on $\mathrm{B}^{+}$is equivalent to the action of $\Gamma$ on $\widetilde{Q}_{s t}$. The latter action is discontinuous on $\widetilde{Q}$ since $\Gamma$ is the covering group of the covering map $\widetilde{Q} \rightarrow Q$. It follows at once that $\Gamma$ is discontinuous on a dense open subset of $\mathrm{B}^{+}$. Since $\Gamma$ acts on $\mathrm{B}^{+}$via isometries, it follows that $\Gamma$ is discrete in Aut $\mathrm{B}^{+}$, the isometry group of $\mathrm{B}^{+}$. Since Aut $\mathrm{B}^{+}$operates transitively on $\mathrm{B}^{+}$with a compact isotropy group, $\Gamma$ operates discontinously on $\mathrm{B}^{+}$.

Corollary (10.19.1). - For any $y \in \widetilde{Q}_{\text {st }}$, the stabilizer $\Gamma_{y}$ of $y$ in $\Gamma$ is a finite group.

Proof. - . Set $p=\tilde{w}_{\mu}(y)$. Then $\mathrm{L}_{y} \approx \Gamma_{p}=\Gamma \cap\left(\text { Aut } \mathrm{B}^{+}\right)_{p}$. Since $\Gamma$ is discrete in Aut $\mathrm{B}^{+}$, and (Aut $\left.\mathrm{B}^{+}\right)_{p}$ is compact, $\Gamma_{p}$ is finite.

Corollary (10.rg.2). - $\widetilde{\mathrm{Q}}$ is the normal covering of $\mathrm{Q}$ universal with respect to the property [R]: for each stable partition $T$ of $\mathrm{S}$ with card $\mathrm{T}=\operatorname{card} \mathrm{S}-\mathrm{I}$, if $k_{\mathrm{T}}$ is defined as in $(9.9)$, the ramification index along $\mathrm{Q}_{\mathrm{T}}$ divides $k_{\mathrm{T}}$.

Proof. -- Let $Q^{*}$ denote the universal covering with the property above. Then the map $Q^{*} \rightarrow \widetilde{Q}$ extends to a covering map of $\widetilde{Q}_{1}$. By Theorem (I0.18.2), $\widetilde{Q}_{1}$ is homeomorphic to the complement in the ball $\mathrm{B}^{+}$of a closed subset of complex codimension 2 and is simply connected. Consequently, $Q^{*} \cong \widetilde{Q}$.

Corollary (ro.19.3). - Above a suitably small neighborhood $\mathrm{U}$ of a point $x \in Q_{\mathrm{sst}}$, each connected component of $\widetilde{\mathrm{Q}} \cap \mathrm{p}^{-1} \mathrm{U}$ is the universal covering of $\mathrm{Q} \cap \mathrm{U}$ with respect to the ramification property $[\mathrm{R}]$.

The proof is the same as the preceding one, with the ball replaced by the trace of a neighborhood of a point in $\overline{\mathbf{B}}^{+}$(in the topology of (5.4)).

Corollary (ro.19.4). - The stabilizer in $\Gamma$ of a point $x$ in the ball is generated by C-reflections.

Proof. -- Choose $\mathrm{U}$ as in Corollary (10.19.3). Then $\mathrm{U} \cap \mathrm{Q}_{1}$ is simply connected. Hence $\pi_{1}(Q \cap U)$ is generated by loops around the ramification locus. These generators yield $\mathbf{C}$-reflections by (9.2), generating $\Gamma_{x}$ if $x \in \mathrm{B}^{+}$.

\section{Finite measure}

In this section, the notation and assumptions (4.0) as well as the assumption $N \geqslant 4$ are continued. We assume moreover that the $\mu^{\prime} s$ are rational. 
(II.x) Let $J \in Q_{8 s t}-Q_{\text {ot }}$ be a semi-stable point of type (S(I), $\left.S(2)\right)$ where $\sum_{s \in \mathrm{S}(1)} \mu_{s}=\mathrm{I}$. Let $a \in \mathrm{S}(\mathrm{I})$ and $b \in \mathrm{S}(2)$. Let $\mathrm{W}$ be the neighborhood of $\mathrm{J}$ in $Q_{88 t}$ lying below the set of all $y \in \mathbf{P}^{\mathrm{S}}$ such that $y(a)=0, y(b)=\infty$ and such that

$$
\sup \{|y(s)| ; s \in \mathbf{S}(1)\} \leq \inf \{|y(s)| ; s \in \mathrm{S}(2)\} \text {. }
$$

Let $\rho: \widetilde{Q}_{\text {sst }} \rightarrow Q_{\text {sst }}$ and let $p \in p^{-1}(J)$. By (8.2.4), $\pi_{1}(Q, o)$ operates on $\widetilde{Q}_{s s t}$, and

$$
\pi_{1}(\mathrm{Q}, o)_{p} \simeq \mathrm{D}_{\mathrm{J}}=\text { image of } \pi_{1}(\mathrm{~W} \cap \mathrm{Q}) \text { in } \pi_{1}(\mathrm{Q}, o) \text {; }
$$

here, $\pi_{1}(Q, 0)_{p}$ denotes the stabilizer of $p$ and $D_{J}$ the decomposition group of $\mathrm{J}$. By (4.5), W $-\{\mathrm{J}\}$ is a simply connected manifold if card $S \geqslant 5$; hence $\pi_{1}(W \cap Q)$ is generated by circuits around the codimension I subspaces $Q_{T}$ for all stable partitions $T$ finer than $\{S(I), S(2)\}$ with $\operatorname{card} T=\operatorname{card} S-I$ if $N \geqslant 5$. By (9.2) the monodromy of such generators are complex reflections which have finite order if $\mu_{1}$ is rational for all $s \in \mathrm{S}$. Thus

(II.I.I) If card $S \geqslant 5$, the quotient $\pi_{1}(Q, o)_{p} / \operatorname{Ker} \theta$ is generated by elements of finite order; here $\theta$ denotes the monodromy action (3.10.2).

(xr.2) Assume that condition (INT) is satisfied. Set $V=\mathrm{H}^{\mathbf{1}}\left(\mathbf{P}_{0}, \mathrm{~L}_{0}\right)$, let $\mathrm{PU}(\mathrm{V})$ denote the projective unitary group on $V$ with respect to the hermitian form of (3.10.2). Let $\mathrm{B}^{+}=\mathrm{B}(\alpha)_{0}^{+}$, i.e. the set of all $v \in \mathrm{V}$ with $(v, v)>0$ modulo $\mathbf{C}^{*}$. Let $\widetilde{w}_{\mu}: \widetilde{Q}_{\mathrm{Bst}} \rightarrow \overline{\mathbf{B}}^{+}$ be the map of Proposition (8.7). Set

$$
\ell=\widetilde{w}_{\mu}(p), \quad \Gamma=\theta\left(\pi_{1}(Q, o)\right) .
$$

By Theorem (10.8. I), $\widetilde{w}_{\mu}$ is a homeomorphism onto an open subset of $\overline{\mathbf{B}}^{+}$. Consequently

$$
\theta\left(\pi_{1}(Q, o)_{p}\right)=\Gamma_{l}
$$

the stabilizer of $\ell$ in $\Gamma$. By (II.I.I) and (10.19.3), we get

(11.2.1) $\Gamma_{l}$ is generated by $\mathbf{C}$-reflections of finite order if $\operatorname{dim} \mathbf{B}^{+}>_{1}$ and by a unipotent element if $\operatorname{dim} \mathbf{B}^{+}=\mathrm{I}$ (cf. $(12 \cdot 3 \cdot 2)$ ).

(xx.3) Let $\delta \in \ell$, let $\mathrm{PU}(\mathrm{V})_{\ell}$ (resp. $\mathrm{PU}(\mathrm{V})_{v}$ ) denote the stabilizer in $\mathrm{PU}(\mathrm{V})$ of $\ell$ (resp. $d_{v}(5 \cdot 3)$ ), and let $N$ denote the unipotent radical of $\mathrm{PU}(\mathrm{V})_{\ell}$. As pointed out in the proof of Proposition (5.5),

$$
\mathrm{PU}(\mathrm{V})_{\mathrm{v}} / \mathrm{N}=\mathrm{U}\left(\mathrm{V}^{\prime \prime}\right)
$$

which is compact, and $\mathrm{PU}(\mathrm{V})_{\ell} / \mathrm{N}=\mathrm{R} \times \mathrm{U}\left(\mathrm{V}^{\prime \prime}\right)$. Hence $\mathrm{PU}(\mathrm{V})_{\mathfrak{v}} / \mathrm{N}$ contains all compact subgroups of $\mathrm{PU}(\mathrm{V})_{\ell} / \mathrm{N}$. Therefore, as is well-known, $\mathrm{PU}(\mathrm{V})_{\mathrm{v}}$ contains all compact subgroups of $\mathrm{PU}(\mathrm{V})_{\ell}$ (because $\mathrm{PU}(\mathrm{V})_{0}$ contains a maximal reductive subgroup and all maximal reductive subgroups of $P U(V)_{\ell}$ are conjugate by an clement of $\left.N\right)$.

From (II.2.I), one infers

(11.3.1) $\quad \Gamma_{\ell} \subset \mathrm{PU}(\mathrm{V})_{\mathrm{v}}$. 
Remark. - A more direct proof of (I .3. I) results from (7.3)-more precisely from the fact that on $\mathrm{W}$, we have a horizontal family of functions $d$. It follows at once that $\mathrm{I}_{p}$ preserves a "distance to $l$ " function $(5 \cdot 3)$.

Theorem (II.4). - Let $\mathrm{S}$ be a finite set, $0<\mu_{\mathrm{s}}<\mathrm{I}, \alpha_{\mathrm{a}}=\exp 2 \pi \sqrt{-\mathrm{I}} \mu_{\mathrm{a}}, \quad \Sigma \mu_{\mathrm{s}}=2$ (all $s \in \mathrm{S})$. Let $\theta: \pi_{1}(\mathrm{Q}, 0) \rightarrow \mathrm{PU}(\mathrm{V})$ be the monodromy action defined in (3.10.2). Assume

(INT) For all $s \neq t$ in $\mathrm{S}$ such that $\mu_{\mathrm{s}}+\mu_{t}<\mathrm{I},\left(\mathrm{I}-\mu_{\mathrm{t}}-\mu_{t}\right)^{-1}$ is an integer.

Then $\Gamma$, the image of 0 , is a lattice in $\mathrm{PU}(\mathrm{V})$.

Proof. - By Theorem (10.19), $\Gamma$ is discrete in PU(V), and by Theorem (10.18.2)

$$
\Gamma \backslash \widetilde{w}_{\mu}\left(\widetilde{Q}_{s s t}\right)=Q_{s s t} \text {, }
$$

where $\widetilde{w}_{\mu}\left(\widetilde{Q}_{\text {sat }}\right)$ is an open subset of $\overline{\mathrm{B}}^{+}$with respect to the topology of $(5 \cdot 4)$. Hence $\Gamma \backslash \widetilde{w}_{\mu}\left(\widetilde{Q}_{\mathrm{Bst}}\right)$ is the union of a compact quotient of a subset of $\mathrm{B}^{+}$and a finite set of neighborhoods $W_{\mathrm{J}}$ (in the topology of $(5 \cdot 4)$ ) of points $\ell_{\mathrm{J}} \in \partial \mathrm{B}^{+}$, where $\ell_{\mathrm{J}} \in \widetilde{w}_{\mu}\left(p^{-1} \mathrm{~J}\right)$, $\rho: \widetilde{Q}_{\text {sst }} \rightarrow Q_{\text {sst }}$, and $J$ varies over $Q_{\text {cusp }}$. By $\S_{4}, Q_{\text {sst }}$ is compact. Any compact subset of $\mathrm{B}^{+}$has finite measure. By (I I.3.I) and Proposition (5.5), the image in $\Gamma \backslash \mathrm{B}^{+}$ of $W_{J}-l_{J}$ has finite measure. It follows that $\Gamma \backslash B^{+}$has finite measure. Thus $\Gamma$ is a lattice in $\mathrm{PU}(\mathrm{V})$.

Lemma (11.5). The subgroup $\Gamma_{\alpha}$ is Zariski-dense in $\mathrm{PU}(\mathrm{V})$.

The proof will be by induction on card S. By (3.10.2), we can lift $\Gamma_{\alpha} \subset \mathrm{PU}(\mathrm{V})$ to a group $\Gamma_{\alpha}^{\prime} \subset \mathrm{U}(\mathrm{V})$ which is generated by pseudo-reflections (cf. (9.2), (12.3.2)); recall the definition: $\gamma$ is a pseudo-reflection if and only if $\gamma-1$ has rank 1 . Hence $\Gamma_{\alpha}^{\prime}$ is irreducible on the linear span $\mathrm{L}$ of the one dimensional subspaces of $\mathrm{V}$ corresponding to its pseudo-reflections.

It is easy to verify that $\mathrm{L}=\mathrm{V}$. Thus $\Gamma_{\alpha}^{\prime}$ stabilizes no proper vector subspace of $\mathrm{V}$.

We start the induction at $\mathrm{N}=4$. Here $\Gamma_{\alpha}$ operates on the complex I-ball as a triangle group, rotating through twice the angle at each vertex of a geodesic triangle. Since $o<\mu_{s}<\mathrm{I}$ and $\sum_{s \in \mathbb{S}} \mu_{s}=2$, the sum of the angles of the geodesic triangle is less than $\pi$ and the orbit of each point in $\mathrm{B}^{+}$under $\Gamma_{\alpha}$ is infinite. Let $\bar{\Gamma}_{\alpha}^{\prime}, \bar{\Gamma}_{\alpha}$ denote the Zariski closure of $\Gamma_{\alpha}^{\prime}, \Gamma_{\alpha}$ in $U(V)$ and $P U(V)$ respectively. The group $\bar{\Gamma}_{\alpha}^{\prime}$ can have no connected normal solvable subgroup non-trivial modulo the center of $U(V)$, otherwise, some $\bar{\Gamma}_{\alpha}^{\prime}$ orbits in $\mathrm{B}^{+}$would be finitc. Consulting the short well known list of closed complex analytic subgroups in $\operatorname{PU}(\mathrm{I}, \mathrm{I})(\approx \operatorname{PGL}(2, \mathbf{R})$, one sees that $\bar{\Gamma}_{\alpha}=\mathrm{PU}(\mathrm{V})$ if card $\mathrm{S}=4$. Suppose now card $\mathrm{S}>4$.

By (6.2I) and two induction hypothesis, $\bar{\Gamma}_{\alpha} \supset \mathrm{PU}(\mathrm{V})$ where $\mathrm{T}$ is a stable partition of $S$ with card $T=\operatorname{card} S-I$ and $V_{T}$ is the corresponding subspace of $V$. Since $\bar{\Gamma}_{\alpha}^{\prime}$ is irreducible on $V$, we infer $\bar{\Gamma}_{\alpha}=\mathrm{PU}(\mathrm{V})$. 


\section{Arithmeticity and Integral Monodromy}

(12.1) Assume that for each $s \in \mathrm{S}, \mu_{\text {, }}$ is a rational number and let $d$ denote the least common denominator of $\left\{\mu_{0} \mid s \in \mathrm{S}\right\}$. Set $\mathbf{F}=\mathbf{Q}(\sqrt[d]{\mathrm{I}})$, $\mathcal{O}$ the ring of integers in $\mathrm{F}$, and $\mathrm{V}=\mathrm{H}^{1}\left(\mathrm{P}_{0}, \mathrm{~L}_{0}\right)$ where $\mathrm{P}_{o}=\mathrm{P}-o(\mathrm{~S})$ as in $(3 . \mathrm{I})$. Then the vector space $\mathrm{V}$ can be defined over the ring $\mathcal{O}$. To see this, let $\mathrm{L}(\mathrm{F})$ (resp. $\mathrm{L}(\mathcal{O})$ ) denote a local subsystem of $L$ on $P_{o}$ (suitably chosen using a base point on $P_{0}$ ) with fiber the subfield $\mathbf{F}$ (resp. subring $\mathcal{O}$ ) of $\mathbf{C}$. The pairing of $\mathrm{L}$ with its complex conjugate induces an $\mathcal{O}$-valued pairing on $\mathrm{L}(\mathcal{O})$. Then one can define cohomology combinatorially as in (2.2) with coefficients in $\mathrm{L}(\mathcal{O})$; one can also define the skew-hermitian cup product

$$
\varphi_{0}: \mathrm{H}^{i}\left(\mathrm{P}_{o}, \mathrm{~L}(\mathcal{O})_{0}\right) \otimes \mathrm{H}_{c}^{2-i}\left(\mathrm{P}_{0}, \check{\mathrm{L}}(\mathcal{O})_{0}\right) \rightarrow \mathrm{H}_{c}^{2}\left(\mathrm{P}_{0}, \mathcal{O}\right) \approx \mathcal{O}
$$

combinatorially; set

$$
\mathrm{V}(\mathcal{O})=\mathrm{H}^{\mathbf{1}}\left(\mathrm{P}_{o}, \mathrm{~L}(\mathcal{O})_{0}\right), \quad \mathrm{V}(\mathrm{F})=\mathrm{H}^{\mathbf{1}}\left(\mathrm{P}_{o}, \mathrm{~L}(\mathrm{~F})_{0}\right)
$$

Define

(12.1.x) $\quad \psi_{\mathcal{O}}(u, v)=j \varphi(u, \bar{v})$,

where $j=b-\bar{b}$ for some $b \in \mathcal{O}-\mathcal{O} \cap \mathbf{R}$. This $\psi_{\mathcal{O}}$ is a hermitian form on $\mathrm{V}$ defined over $\mathcal{O}$ and may be identified with the form defined in (2.18), up to a real scalar factor.

We lift the map $\theta: \pi_{1}(\mathbf{Q}, 0) \rightarrow \mathrm{PU}(\mathrm{V})$ of $(3.10 .2)$ via a local system $\check{\mathrm{L}}$ on $\mathrm{M}(c)$ to $\theta^{\prime}: \pi_{1}(\mathrm{M}(c), 0) \rightarrow \mathrm{U}(\mathrm{V})$ by (3.14). The image of $\theta^{\prime}$ is in $\mathrm{U}(\mathrm{V})(\mathcal{O})$. Set

$$
\text { (12.1.2) } \quad \Gamma_{\alpha}=\operatorname{Im} \theta, \quad \Gamma_{\alpha}^{\prime}=\operatorname{Im} \theta^{\prime}
$$

where $\alpha=\left\{\exp 2 \pi i \mu_{s} \mid s \in \mathrm{S}\right\}$. Then

(12.1.3) $\quad \Gamma_{x}^{\prime}$ stabilizes $\mathrm{V}(\mathcal{O})$.

(12.2) We collect here some remarks and definitions pertaining to arithmeticity of lattices.

Let $\Gamma$ be a Zariski-dense subgroup of $\mathrm{G}(k)$, for $\mathrm{G}$ an adjoint connected semi-simple algebraic group $\mathrm{G}$ over $k$, a field of characteristic o. Set $\mathrm{E}=\mathbf{Q}[\operatorname{Tr}$ Ad $\Gamma$ ], the field spanned over $\mathbf{Q}$ by $\{\operatorname{Tr} \operatorname{Ad} \gamma \mid \gamma \in \Gamma\}$.

\section{Proposition (12.2. r)}

(i) The group $\mathrm{G}$ has a faithful matrix representation $p$ such that $\rho(\Gamma) \subset \mathrm{GL}_{n}(\mathrm{E})$ (and hence $\mathrm{G}$ has $\mathrm{E}$ as a field of definition).

(ii) $\mathrm{E}$ remains unchanged when $\Gamma$ is replaced by a commensurable subgroup of $\mathrm{G}$.

Proof. - Let T denote the function $g \mapsto \operatorname{Tr}$ Ad $g$ on G, and let W denote the C-linear span of the left G-translates of T (we define $(x \cdot f)(y)=f(y x)$ for any function $f: \mathbf{G} \rightarrow \mathbf{C}$, $x$ and $y$ in $\mathrm{G})$. Inasmuch as $\mathrm{T}$ is a sum of matrix coefficients, $\mathrm{W}$ is finite dimensional. Since $\Gamma$ is Zariski dense in $G$, there is a finite set of elements $x_{1}, \ldots, x_{n}$ in $\Gamma$ such that 
$\beta=\left\{x_{1}, T, \ldots, x_{n} . T\right\}$ is a base of $W$. Let $P_{\omega}: G \rightarrow$ Aut $W$ be the representation given by $x \mapsto x . f$ for $x \in \mathrm{G}, f \in \mathrm{W}$. It is well known that the representation $P_{\omega}$ is faithful ([10], Theorem (2.1), p. 123). Let $p(x)$ denote the matrix of $p_{\omega}(x)$ with respect to the base $\beta$. Then $\rho(x)$ has all its entries in the field $\mathrm{F}$ for all $x \in \Gamma$.

To prove (ii) it clearly suffices to consider the case that $\Gamma_{0}$ is a subgroup of $\Gamma$ of finite index. Replacing $\Gamma_{0}$ by $\bigcap_{x \in \Gamma} x \Gamma x^{-1}$, we can suppose moreover that $\Gamma_{0}$ is normal in $\Gamma$. Then $\Gamma_{0}$ is also Zariski-dense in G. Set $E_{0}=\mathbf{Q}\left[\operatorname{Tr} A d \Gamma_{0}\right]$ and select the base $\beta=\left\{x_{1}, \mathrm{~T}, \ldots, x_{n} . \mathrm{T}\right\}$ with $x_{i} \in \Gamma_{0} \quad(i=1, \ldots, n)$. Let $\sigma: \mathrm{E} \rightarrow \mathbf{C}$ be a monomorphism which is the identity on $E_{0}$. To prove (ii), it suffices to prove that $\sigma$ leaves each element of $\mathrm{E}$ fixed. For any $y \in \Gamma$ and $x \in \Gamma_{0}$, we have $y x y^{-1} \in \Gamma_{0}$. Hence, by $(12.2 .1)$,

$$
\rho(y x y)^{-1}={ }^{\circ} p\left(y x y^{-1}\right)={ }^{\circ} p(y){ }^{\circ} p(x){ }^{\circ} p(y)^{-1},
$$

that is $\rho(y) \rho(x) \rho(y)^{-1}={ }^{0} \rho(y) \rho(x){ }^{0} \rho(y)^{-1}$. Hence for all $y \in \Gamma_{0}, \rho(y)^{-1 \sigma} \rho(y)$ centralizes $\rho\left(\Gamma_{0}\right)$ and therefore $\rho(G)$ since $\Gamma_{0}$ is Zariski-dense in $G$. The center of $\rho(G)$ is $\mathrm{I}$ and consequently ${ }^{\circ} \rho(y)=\rho(y)$ for all $y \in \mathrm{G}$. Thercfore $\sigma$ fixes each element of $\mathrm{E}$.

(12.2.2) Take $p$ as in (12.2.1). The Zariski closure of $p(\Gamma)$ in $G_{n}(E)$ is an algebraic group over E. Because taking Zariski closure commutes with field extensions it is a form of $\mathrm{G}$ over $\mathrm{E}$. This provides the group $\mathrm{G}$ with an E-structure, called the natural $\mathrm{E}$-structure of $\mathrm{G}$. If $\rho^{\prime}$ is another faithful matrix representation of $G$ with $\rho^{\prime}(\Gamma) \subset \mathrm{GL}_{m}(E)$, it leads to the same E-structure. To check this, one compares $\rho$ to $\rho^{\prime}$ via $\rho \oplus \rho^{\prime}$.

If $F$ is a subfield of $k$ and $G_{F}$ an $F$-structure on $G$ for which $\Gamma \subset G_{F}(F)$, the field of traces $E$ is contained in $F$ and, by the above construction applied to $G_{F}$ over $F$, the given $F$-structure on $\mathrm{G}$ is deduced from the natural E-structure.

(12.2.3) Let A be a semi-simple algebraic linear group defined over the field $\mathbf{Q}$ of rational numbers, let $\mathrm{V}$ be a finite dimensional vector space defined over $\boldsymbol{Q}$, and $\rho: A \rightarrow G L(V)$ a faithful rational representation defined over $\mathbf{Q}$. By a theorem of Borel-Harish-Chandra [3], the subgroup $\Gamma=\rho^{-1}\left(G L\left(V_{z}\right)\right)$ is a lattice in $A(R)$ for any lattice $V_{z}$ in $V_{Q}$; that is, $\Gamma$ is a discrete subgroup of $A(R)$ and $A(\mathbf{R}) / \Gamma$ has finite measure.

(12.2.4) Let $G$ be an adjoint connected semi-simple real Lie group. It is the topological connected component $\mathbf{G}(\mathbf{R})^{0}$ of $\mathbf{G}(\mathbf{R})$, for $\mathbf{G}$ an adjoint connected semi-simple algebraic group over $\mathbf{R}$. By definition, a subgroup $\Gamma$ of $G$ is arithmetic in $G$ if and only if there exists an algebraic group $A$ over $\mathbf{Q}$, a compact group $K$ and an analytic isomorphism $\theta$ of $\mathbf{A}(\mathbf{R})^{0}$ onto $G \times K$ such that $\theta\left(\mathbf{A}(\mathbf{Z}) \cap A(\mathbf{R})^{0}\right)$ has its projection into $G$ commensurable to $\Gamma$. The group $A$ is necessarily reductive. It can be assumed connected adjoint semi-simple; namely, replace $A$ by $A^{0} /$ center $\left(A^{0}\right)$. The group $A$ is then a product of $\mathbf{Q}$-simple groups $A_{i}$ (cf. [23], p. $4^{6}(3.1 .2)$ ). If $G$ is a simple non- 
compact Lie group, it follows from the definition that all $A_{i}$ but one, $A_{1}$, are such that $A_{i}(\mathbf{R})$ is compact. Replacing $A$ by $A_{1}$, one can then assume that $A$ is $\mathbf{Q}$-simple, i.e. of the form (cf. [23], p. 46 (3.I.2))

$$
A=\operatorname{Restr}_{F / Q} B
$$

for $B$ an absolutely simple group over a finite extension $F$ of $\mathbf{Q}$. One has $\mathbf{F} \otimes \mathbf{R}=\prod_{v} F_{v}$ (product of the completions of $F$ at the infinite places $v$ ) and $A(\mathbf{R})=B(F \otimes \mathbf{R})=\prod_{v} B\left(F_{v}\right)$. The existence of $\theta$ as in (12.2.4) amounts to saying that for all $v$ but one, $v_{1}, B\left(F_{v}\right)$ is compact (hence if $v \neq v_{1}$ the place $v$ is a real place) and that $B\left(F_{v_{1}}\right)^{0} \simeq G$, the isomorphism carrying $B(\mathcal{O})$ to a subgroup of $G$ commensurable to $\Gamma$. If $G$ is an absolutely simple Lie group, the place $v_{1}$ is a real place, $\mathrm{F}$ is totally real, and $\mathrm{B}$ is an F-form of $\mathbf{G}$, $F$ being identified with a subfield of $\mathbf{R}$ via $v_{1}$.

(12.2.5) Assume that $G$ is absolutely simple non compact. In our application, it is $\mathrm{PU}(\mathrm{r}, \mathrm{N}-3)$. By the Borel density theorem, any arithmetic $\Gamma$ in $\mathrm{G}$ is Zariski dense. If $A=\operatorname{Restr}_{F / a} B$ is as above, with $F \subset \mathbf{R}, B$ an $F$-form of $G$, and $\Gamma$ a subgroup commensurable to $B\left(\mathcal{O}_{\mathrm{F}}\right)$, then by (12.2.I), (12.2.2) $\mathrm{F} \supset \mathrm{E}$ and the F-form $\mathrm{B}$ is deduced from the natural $\mathrm{E}$-structure of $\mathbf{G}$ defined by $\Gamma$. For all the real places $\boldsymbol{v}$ of $\mathbf{F}$ above the identity embedding of $\mathrm{E}$, the $\mathbf{G}\left(\mathrm{F}_{\mathrm{v}}\right)$ are isomorphic, hence non compact. There can hence be only one such $v$. Hence $\mathrm{F}=\mathrm{E}$. We conclude:

(12.2.6) Assume $G$ is an adjoint connected absolutely simple non compact Lie group. Then a subgroup $\Gamma \subset G$ is arithmetic if and only if

a) the field of traces $\mathrm{E}$ is totally real;

b) for each embedding $\sigma$ of $E$ in $\mathbf{R}$ distinct from the identity embedding $\left({ }^{\circ} \mathbf{G}\right)(\mathbf{R})$ is a compact group (i.e. the real group $\mathbf{G} \otimes_{E, \sigma} \mathbf{R}$ deduced from the natural E-structure of $\mathbf{G}$ is compact). $\mathbf{G}$ being as in (12.2.4);

c) $\Gamma$ is commensurable with $\mathbf{G}\left(\mathcal{O}_{\mathrm{E}}\right)$.

(12.2.7) Let $G$ be an adjoint connected absolutely simple non compact Lie group, and let $\Gamma$ be a lattice in $G$. Assume a totally real number field $F \subset \mathbf{R}$, and a form $\mathbf{G}_{\boldsymbol{r}}$ of $\mathbf{G}$ over $\mathbf{F}$ are given, such that a subgroup of finite index of $\Gamma$ is contained in $\mathbf{G}\left(\mathcal{O}_{\mathrm{F}}\right)$. Then the field $\mathrm{E}=\mathbf{Q}[\operatorname{Tr} \mathrm{Ad} \Gamma]$ is contained in $\mathrm{F}$ by (12.2.I).

Corollary (12.2.8). - A lattice $\Gamma \subset \mathrm{G}$ is arithmetic in $\mathrm{G}$ if and only if, for each embedding $\sigma$ of $\mathrm{F}$ in $\mathrm{R}$, not inducing the identity embedding of $\mathrm{E}$ in $\mathrm{R}$, the real group $\mathbf{G}_{\mathrm{F}} \otimes_{\mathrm{F}, \mathrm{a}} \mathrm{R}$ is compact (i.e. ${ }^{\circ} \mathbf{G}(\mathrm{R})$ is a compact group).

Proof. - Since EC F, a) of $(12.2 .6)$ is implied by the assumption that $\mathrm{F}$ is totally real. By (12.2.2), the given F-structure of $\mathbf{G}$ is deduced from the natural E-structure and $\Gamma \subset \mathbf{G}(\mathrm{E})$. The condition in (12.2.8) amounts to $b)$ of (12.2.6). Assume it 
holds. Then, $\mathbf{G}\left(\mathcal{O}_{\mathrm{E}}\right)$ is an arithmetic lattice in $\mathbf{G}(\mathbf{R})$. A subgroup of finite index in $\Gamma$ lies in $\mathbf{G}(\mathbf{E}) \cap \mathbf{G}\left(\mathcal{O}_{F}\right)$ and hence $\mathbf{G}\left(\mathcal{O}_{\mathrm{E}}\right)$ contains a subgroup $\Gamma^{\prime}$ of finite index in $\Gamma$. Both $\Gamma^{\prime}$ and $\mathbf{G}\left(\mathcal{O}_{E}\right)$, being lattices in $\mathbf{G}(\mathbf{R})$, have finite covolume; hence the index of $\Gamma^{\prime}$ in $\mathrm{G}\left(\mathcal{O}_{E}\right)$ is finite. Condition $\left.c\right)$ of $(12.2 .6)$ follows.

The criterion (12.2.8) will be applied to test the arithmeticity of $\Gamma_{\alpha}$ in $P U\left(V, \psi_{0}\right)$.

(12.3) We shall need more explicit information about monodromy than given in (9.2) in order to compute $\boldsymbol{Q}\left[\operatorname{Tr} \Gamma_{\alpha}^{\prime}\right]$, where $\Gamma_{\alpha}^{\prime}$ is as in (12.1.2). The result needed is (12.5. I). Actually, by consulting the explicit lists in $\S 14$, one can obtain the required information in a case-by-case inspection except in three of the cases.

For the remainder of $\oint_{12}$, we assume $0<\mu_{8}<I$ and $\mu_{z} \in \mathbf{Q}$ for all $s \in \mathbf{S}$.

Let $S=S_{1} \cup S_{2}$ with card $S_{2}=1$, let $T_{1}, T_{2}$ be trees as in $(2.5)$ with the vertices of $T_{i}$ in $S_{i}(i=1,2)$, and let $\beta: T_{1} \amalg T_{2} \rightarrow P$ be an embedding with $\beta \mid S=0$, the base point of $M$.

Without loss of generality, we can assume that $T_{1}$ is homeomorphic to the interval $0 \leq x \leq 1$

Fix an orientation on $T_{1}$, let $s_{1}, \ldots, s_{\mathrm{N}-1}$ denote the vertices of $T_{1}$ taken in order, and let $a_{i}$ denote the oriented edge from $s_{i}$ to $s_{i+1}(1 \leq i \leq N-2)$. Let $\hat{T}$ denote the cone over $T_{1} \amalg T_{2}$ with apex $\wedge$ and denote by $\beta$ also an extension of $\beta$ to an embedding of $\hat{\mathrm{T}}$ in $\mathrm{P}$. Since $\hat{\mathrm{T}}-\left\{s_{1}, \ldots, s_{\mathrm{N}}\right\}$ is simply connected, the pull-back $\beta^{*} \breve{\mathrm{L}}$ may be identified with the constant system $\mathbf{C}$ on it, and we can choose $\ell\left(a_{i}\right) \in \mathrm{H}^{0}\left(a_{i}, \beta^{*} \check{L}\right)$ for each $i$ so that

$$
\ell\left(a_{i}\right) \cdot \beta\left|a_{i} \approx-\mathrm{I} \cdot \beta\right| \hat{s}_{\mathrm{i}}+\mathrm{I} \cdot \beta \mid \hat{s}_{\mathrm{i}+1}, \quad \mathrm{I} \leq i \leq \mathrm{N}-2
$$

where $\hat{s}_{i}$ is the arc from $\wedge$ to $s_{i}$ and $\approx$ denotes homology. Let $w_{i}$ denote the element in $\mathrm{H}_{1}^{\mathrm{H}}\left(\mathrm{P}_{o}, \breve{\mathrm{L}}(\mathcal{O})\right)$ determined by $\ell\left(a_{i}\right) . \beta \mid a_{i}$; then as in $(2.5)\left\{w_{1}, \ldots, w_{\mathrm{N}-2}\right\}$ is a basis. Let $\gamma_{i, j}$ denote the monodromy effected on $\mathrm{H}_{1}^{\mathrm{lf}}\left(\mathbf{P}_{o}, \check{\mathrm{L}}\right)$ by moving $o\left(s_{j}\right)$ along a path close to $\beta\left(T_{1}\right)$ disjoint from $\beta(\hat{T}) \cup D$, except at its initial point, where $D_{r}$ is a small disc centered at $o\left(s_{i}\right)$, then making one positive turn around $\partial \mathrm{D}_{r}$, and then retracing the path to $o\left(s_{j}\right)$.

Then

Lemma (12.3.2). - Set $w_{k}^{0}=\gamma_{1,2} w_{k}$ and $w_{k}^{\prime}=\gamma_{1,3} w_{k}, k=1, \ldots, N-2$.

and

$$
\begin{aligned}
& w_{1}^{0}=\bar{\alpha}_{1} \bar{\alpha}_{2} w_{1}, \\
& w_{2}^{0}=\bar{\alpha}_{2}\left(1-\bar{\alpha}_{1}\right) w_{1}+w_{2}, \\
& w_{k}^{0}=w_{k}, \quad k>2, \\
& w_{1}^{\prime}=\bar{\alpha}_{3} w_{1}+\alpha_{2}\left(\bar{\alpha}_{3}-1\right) w_{2}, \\
& w_{2}^{\prime}=\bar{\alpha}_{2} \bar{\alpha}_{3}\left(\bar{\alpha}_{1}-1\right) w_{1}+\left(\bar{\alpha}_{3} \alpha_{1}-\bar{\alpha}_{3}+\mathrm{I}\right) w_{2}, \\
& w_{3}^{\prime}=\bar{\alpha}_{2} \bar{\alpha}_{3}\left(1-\bar{\alpha}_{1}\right) w_{1}+\bar{\alpha}_{3}\left(1-\bar{\alpha}_{1}\right) w_{2}+w_{3}, \quad \text { if } \mathrm{N}-3>\mathrm{I}, \\
& w_{k}^{\prime}=w_{k}, \quad k \geq 3 .
\end{aligned}
$$


Proof. - Let $c$ denote the unique element in the $\mathrm{S}_{\mathbf{2}}$. Introduce the local system $\mathrm{L}(c)$ of (3.I5) on $P_{\mathbf{M}(c)}$. Without loss of generality, we can assume that our base point $o \in \mathrm{M}(c)$ i.e. $o(c)=\infty$.

Set $d=\beta(\wedge)$. Without loss of generality we can choose the embedding $\alpha$ of $\hat{T}$ so that $\inf _{s \in \mathrm{S}_{1}}|d-o(s)|>3$ diam $o\left(\mathrm{~S}_{1}\right)$ (here we are identifying $\mathrm{P}$ with $\mathbf{P}$ via a coordinate $z$ as in $(3.15))$.

The effect of horizontal transport of $\left\{w_{1}, \ldots, w_{\mathrm{N}-2}\right\}$ under variation of embeddings of $\mathbf{S}$ in $\mathbf{P}$ is given by an isotopy of $\mathbf{P}$ (cf. (3.6)). In particular, $\gamma_{i, j}$ arises from an isotopy $\left\{\eta_{i, j}^{i} ; 0 \leq t \leq 1\right\}$ of $\mathrm{P}$ which twists $o\left(s_{j}\right)$ one positive turn around $o\left(s_{i}\right)$ along the path described above; here $\eta_{i, j}^{0}=$ identity and $\eta_{i, j}^{1} \mid o(S)=$ identity. The isotopy $\eta_{i,}^{i}$ can be performed so that the only points which move lie in a disc containing $o\left(\mathrm{~S}_{1}\right)$ of diameter less than 2 diam $o\left(\mathrm{~S}_{1}\right)$. Since under the isotopy no point of $o\left(\mathrm{~S}_{1}\right)$. turns around $d$, one sees by inspection of the definition of $\mathrm{L}(c)$ that the restriction of the dual local system $\check{\mathrm{L}}(c)$ to the subset $\left\{\left(d \times \eta_{i, j}^{i} o\left(\mathrm{~S}_{1}\right)\right) ; 0 \leq t \leq \mathrm{I}\right\}$ of $\mathrm{P}_{\mathrm{M}(c)}$ has a trivializing nonzero section $\check{e}, \mathrm{I} \leq i, j \leq \mathrm{N}-\mathrm{I}$. Consequently, for each $i, j \leq \mathrm{N}-\mathrm{I}, \check{e}(d)$ returns to its initial value after horizontal transport via $\eta_{i, j}^{b}, \quad 0 \leq t \leq \mathrm{r}$.

Set $v_{k}=\check{e} \cdot \beta \mid \hat{s}_{k}$ and $v_{k}^{\prime}=\eta_{i, j}^{1}\left(v_{k}\right), \mathrm{I} \leq i, j, k \leq \mathrm{N}-\mathrm{I}$. In view of (12.3.1), $w_{k}^{\prime} \approx-v_{k}^{\prime}+v_{k+1}^{\prime}$ when the section $\check{e}$ is taken to have the same value on $d$ as the section denoted $\ell$ in (12.3.1); we choose $\check{e}$ in this way. The argument above shows that the initial segment of the $\breve{\mathrm{L}}$ valued singular chain $v_{k}$ is unchanged by the map $\eta_{i j}^{1}$, $\mathrm{I} \leq i, j, k \leq \mathrm{N}-\mathrm{I}$.

a)

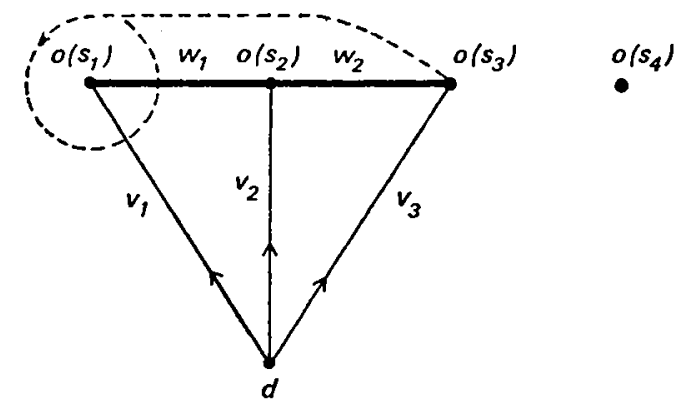

b)

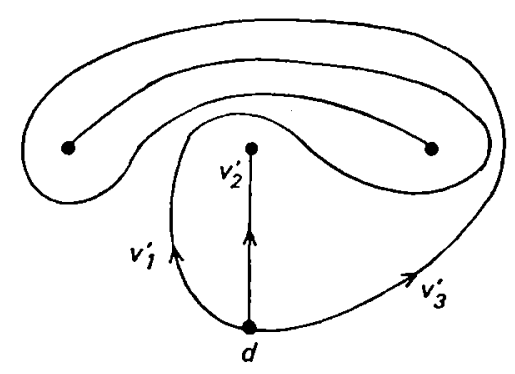


The effect of the isotopy $\eta$ corresponding to $\gamma_{1,3}$ is pictured in diagrams $a$ ) and $b$ ). Let " " denote " homotopic in $\mathrm{P}_{0}$ ". Then

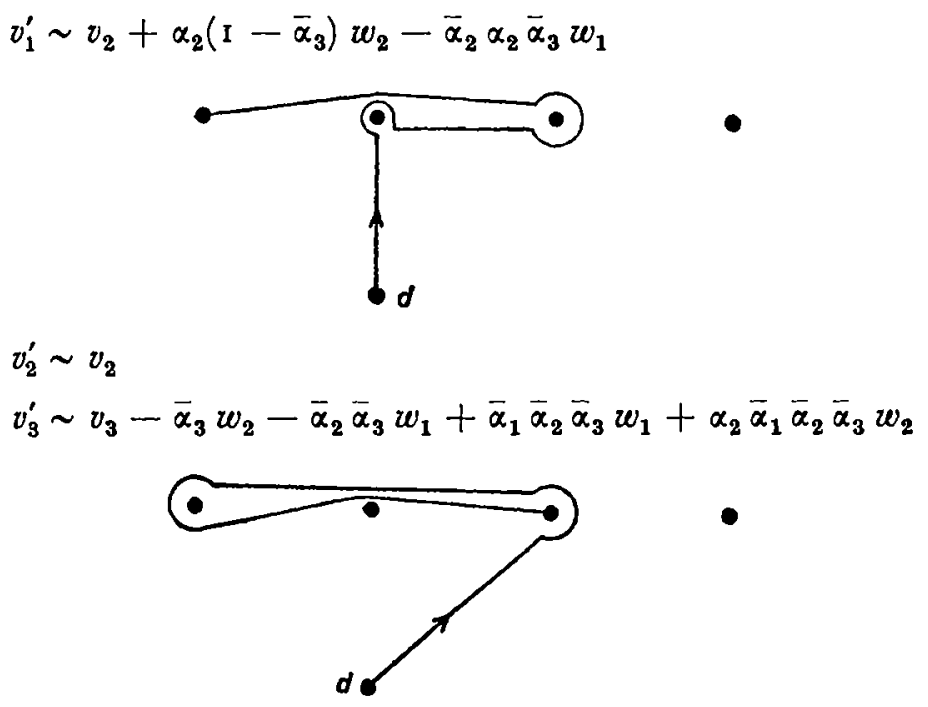

$v_{k}^{\prime} \sim v_{k}$ for $k \geq 4$

Consequently

$$
\begin{aligned}
w_{1}^{\prime} \sim-v_{1}^{\prime}+v_{2}^{\prime} & \approx \bar{\alpha}_{3} w_{1}+\bar{\alpha}_{2}\left(\alpha_{3}-1\right) w_{2}, \\
w_{2}^{\prime} \sim-v_{2}^{\prime}+v_{3}^{\prime} & \sim-v_{2}+v_{3}+\bar{\alpha}_{2} \bar{\alpha}_{3}\left(\bar{\alpha}_{1}-1\right) w_{1}+\left(\bar{\alpha}_{1} \bar{\alpha}_{3}-\bar{\alpha}_{3}\right) w_{2} \\
& \approx \bar{\alpha}_{2} \bar{\alpha}_{3}\left(\bar{\alpha}_{1}-1\right) w_{1}+\left(1-\bar{\alpha}_{3}+\bar{\alpha}_{1} \bar{\alpha}_{3}\right) w_{2}, \\
w_{3}^{\prime} \sim-v_{3}^{\prime}+v_{4}^{\prime} & \sim-v_{3}+v_{4}+\bar{\alpha}_{2} \bar{\alpha}_{3}\left(1-\bar{\alpha}_{1}\right) w_{1}+\left(\bar{\alpha}_{3}-\bar{\alpha}_{1} \bar{\alpha}_{3}\right) w_{2} \\
& \approx \bar{\alpha}_{2} \bar{\alpha}_{3}\left(1-\bar{\alpha}_{1}\right) w_{1}+\bar{\alpha}_{3}\left(1-\bar{\alpha}_{1}\right) w_{2}+w_{3} .
\end{aligned}
$$

The second part of $(12.3 .2)$ follows at once. The proof for $\gamma_{1,2}$ is similar.

(12.4) For $\mathrm{N}=4$, calculations as above yield for matrices with respect to the basis $\left\{w_{1}, w_{2}\right\}$

$$
\begin{aligned}
& \gamma_{1,2}=\left(\begin{array}{cc}
\bar{\alpha}_{1} \bar{\alpha}_{2} & \bar{\alpha}_{2}\left(1-\bar{\alpha}_{1}\right) \\
0 & 1
\end{array}\right), \quad \gamma_{2,3}=\left(\begin{array}{cc}
1 & 0 \\
1-\bar{\alpha}_{3} & \bar{\alpha}_{2} \bar{\alpha}_{3}
\end{array}\right), \\
& \gamma_{1,3}=\left(\begin{array}{cc}
\bar{\alpha}_{3} & \bar{\alpha}_{2} \bar{\alpha}_{3}\left(\bar{\alpha}_{1}-1\right) \\
\alpha_{2}\left(\bar{\alpha}_{3}-1\right) & \bar{\alpha}_{1} \bar{\alpha}_{3}-\bar{\alpha}_{3}+1
\end{array}\right)
\end{aligned}
$$

and we easily verify that

$$
\text { (12.4.x) } \quad \gamma_{1,2} \cdot \gamma_{2,3} \cdot \gamma_{13}=\overline{\alpha_{1} \alpha_{2} \alpha_{3}} \text {. }
$$

This shows that our lift $\theta^{\prime}: \pi_{1}(\mathrm{M}(c), 0) \rightarrow \mathrm{U}(\mathrm{V})$ of $\theta: \pi_{1}(\mathbf{Q}, 0) \rightarrow \mathrm{PU}(\mathrm{V})$ cannot be factored through $\pi_{1}(M, 0) \rightarrow U(V)$ if $\mu_{4} \neq 1 / 2$, since the center of $\pi_{1}(M)$ is $\pi_{1}(\operatorname{PGL}(2))=\mathbf{Z}_{2}$. 
Lemma (12.5). - As in (12.1), set $\mathrm{F}=\mathbf{Q}(\sqrt[d]{\mathrm{I}})$ and $\Gamma_{\alpha}^{\prime}=\operatorname{Im} \theta^{\prime}$. Then

(12.5.1) $\quad \mathbf{Q}\left[\operatorname{Tr} \Gamma_{\alpha}^{\prime}\right]=\mathrm{F}$

(12.5.2) $\quad \mathbf{Q}\left[\operatorname{Tr} A d \Gamma_{\alpha}^{\prime}\right]=\mathrm{F} \cap \mathbf{R}$ if $\operatorname{card} \mathrm{S}>4$.

Proof of (12.5.1). - By (12.1.2), $\mathbf{Q}\left[\operatorname{Tr} \Gamma_{\alpha}^{\prime}\right] \subset$ F. By (9.2) $\mathbf{Q}\left[\operatorname{Tr} \Gamma_{\alpha}^{\prime}\right]$ contains $\alpha_{\varepsilon} \alpha_{z^{\prime}}$, for each distinct pair $s, s^{\prime} \in \mathrm{S}$. It follows that $\mathbf{Q}\left(\operatorname{Tr} \Gamma_{\alpha}^{\prime}\right) \supset \mathbf{Q}(\sqrt[n]{1})$ where $n$ is the least common denominator of $\left\{\mu_{s}+\mu_{s^{\prime}} \mid s, s^{\prime} \in \mathrm{S}, s=s^{\prime}\right\}$. For any 3 distinct elements $s_{1}, s_{2}, s_{3}$ of $\mathrm{S}$, we have

$$
\mu_{s_{1}}-\mu_{s_{1}}=\left(\mu_{s_{1}}+\mu_{s_{2}}\right)-\left(\mu_{s_{2}}+\mu_{s_{2}}\right) \text {. }
$$

Hence for any distinct $s, s^{\prime}$ in $S, Q\left[\operatorname{Tr} \Gamma_{x}^{\prime}\right]$ contains $\alpha_{a^{\prime}} \alpha_{a}^{ \pm 1}$.

We choose a basis $\left\{w_{1}, \ldots, w_{\mathrm{N}-2}\right\}$ in $\mathrm{H}_{1}^{\prime \prime}\left(\mathrm{P}_{0}, \breve{\mathrm{L}}\right)$ as in $(12 \cdot 3)$. Then $\gamma_{1,3} \cdot \gamma_{1,2}$ has as matrix with respect to this basis the upper $2 \times 2$ diagonal of

$$
\left(\begin{array}{ccc}
\bar{\alpha}_{3} & \bar{\alpha}_{2} \bar{\alpha}_{3}\left(\bar{\alpha}_{1}-1\right) & \bar{\alpha}_{1} \bar{\alpha}_{3}\left(1-\bar{\alpha}_{1}\right) \\
\alpha_{2}\left(\bar{\alpha}_{3}-1\right) & \bar{\alpha}_{3} \bar{\alpha}_{1}-\bar{\alpha}_{3}+1 & \bar{\alpha}_{3}\left(1-\bar{\alpha}_{1}\right) \\
0 & 0 & 1
\end{array}\right)\left(\begin{array}{ccc}
\bar{\alpha}_{2} \bar{\alpha}_{1} & \bar{\alpha}_{2}\left(1-\bar{\alpha}_{1}\right) & 0 \\
0 & 1 & 0 \\
0 & 0 & 1
\end{array}\right) .
$$

The diagonal terms of the product are

$$
\bar{\alpha}_{3} \bar{\alpha}_{2} \bar{\alpha}_{1}, \ldots-\left(\bar{\alpha}_{1}-1\right)\left(\bar{\alpha}_{3}-1\right)+\bar{\alpha}_{3} \bar{\alpha}_{1}-\bar{\alpha}_{3}+1,1, \ldots,
$$

therefore, for any $s_{1}, s_{2}, s_{3} \in \mathrm{S}_{1}$ we get

$$
\operatorname{Tr} \gamma_{1,3} \gamma_{1,2}=\bar{\alpha}_{1} \bar{\alpha}_{2} \bar{\alpha}_{3}+\bar{\alpha}_{1}+N-4=\bar{\alpha}_{1}\left(\bar{\alpha}_{2} \bar{\alpha}_{3}+1\right) \bmod \mathbf{Q} .
$$

Hence $\bar{\alpha}_{1} \in \mathbf{Q}\left[\operatorname{Tr} \Gamma_{\alpha}^{\prime}\right]$, provided that $\alpha_{2} \alpha_{3} \neq-\mathrm{I}$ for some $s_{2}, s_{3} \in \mathrm{S}_{1}-\left\{s_{1}\right\}$. This proviso fails only if $\mu_{1}=1 / 4$ for all $s$ in $S_{1}$; in this case, replace $S_{1}$ by a partition $T_{1}$ with card $T_{1}=$ card $S_{1}$ (cf. $(6.2 .1)$ ), and in the corresponding subgroup of $\Gamma_{\alpha}^{\prime}$, the proviso holds. It follows that $\alpha_{\mathbf{q}} \in \mathbf{Q}\left[\operatorname{Tr} \Gamma_{\alpha}^{\prime}\right]$ for all $s \in \mathrm{S}$. This proves (12.5.1).

Proof of (12.5.2). - Set $\mathbf{E}=\mathbf{Q}\left[\operatorname{Tr} A d \Gamma_{\alpha}^{\prime}\right]$ and $\mathscr{A}=\mathbf{C}\left[\operatorname{Int} \Gamma_{\alpha}^{\prime}\right]$ : the C-linear span of the automorphisms Int $\gamma: m \rightarrow{ }_{i} m \gamma^{-1}$ with $\gamma \in \Gamma_{\alpha}^{\prime}$, where $m \in \operatorname{Hom}_{\mathrm{c}}(\mathrm{V}, \mathrm{V})$ and $\mathrm{V}$ is as in $(12.1)$. Let $\sigma \in \operatorname{Gal}(\mathrm{F} / \mathrm{E})$. Inasmuch as $\operatorname{Tr} \operatorname{Int} g=1+\operatorname{Tr} \operatorname{Ad} g$ for any $g \in G L(V)$, it follows by definition of $E$ that for any $\gamma \in \Gamma_{\alpha}^{\prime}$

$$
\text { (12.5.3) } \quad \operatorname{Tr}\left(\operatorname{Int}{ }^{\circ} \gamma\right)={ }^{\circ} \operatorname{Tr}(\operatorname{Int} \gamma)=\operatorname{Tr}(\operatorname{Int} \gamma) \text {. }
$$

It follows from this that

(12.5.4) Int $\gamma \rightarrow{ }^{\circ}$ Int $\gamma$ extends to a C-linear map $\varphi$ of $\mathscr{A}$.

For $\Sigma c_{\gamma} \gamma=0$ with $c_{\gamma} \in \mathbf{C}, \gamma \in \operatorname{Int} \Gamma_{\alpha}^{\prime}$ implies that for any $\left\{d_{\gamma}\right\}$ with $d_{\gamma} \in \mathbf{C}$,

$$
\begin{aligned}
\operatorname{Tr}\left(\Sigma c_{\gamma}{ }^{\sigma} \gamma\right)\left(\Sigma d_{\gamma}{ }^{\sigma} \gamma\right) & =\operatorname{Tr} \Sigma c_{\gamma} d_{\gamma^{\prime}}{ }^{\sigma}\left(\gamma \gamma^{\prime}\right) \\
& =\operatorname{Tr} c_{\gamma} d_{\gamma^{\prime}}\left(\gamma \gamma^{\prime}\right) \\
& =\left(\operatorname{Tr} \Sigma c_{\gamma} \gamma\right)\left(\Sigma d_{\gamma} \gamma\right)=0 .
\end{aligned}
$$


The group Int $\Gamma_{\alpha}^{\prime}$ is Zariski-dense in Int GL(V). Consequently $\mathscr{A}$ coincides with the $\mathbf{C}$-linear span of Int $\mathrm{GL}(\mathrm{V})$; thus $\mathscr{A}$ is an associative algebra acting irreducibly on the subspace $\mathscr{P}$ of trace o elements in $\mathrm{Hom}_{\mathfrak{c}}\left(\mathrm{V}^{r}, \mathrm{~V}\right)$ and stabilizing the line $\mathrm{C}$. I. Since a field automorphism preserves Zariski-density, the $\mathbf{C}$-linear span of 'Int $\Gamma_{\alpha}^{\prime}$ is also $\mathscr{A}$. Therefore

$$
\operatorname{Tr}\left(\left(\Sigma c_{Y}{ }^{\circ} \gamma\right) \cdot m\right)=0 \text { for all } m \in \mathscr{A}
$$

Since $\operatorname{Tr}$ is non-degenerate on $\mathscr{A}$ it follows that $\Sigma c_{\gamma}{ }^{\sigma} \gamma=0$. This implies (12.5.4).

Clearly $\varphi$ preserves products and is an algebra isomorphism. Since $\mathscr{A} \approx \operatorname{Hom}_{\mathrm{c}}(\mathscr{S}, \mathscr{S}), \mathscr{A}$ is a simple associative algebra. The map $\phi$ is an algebra isomorphism and therefore $\varphi$ maps Int GL(V) isomorphically onto the Zariski closure of Int ${ }^{\circ} \Gamma_{\alpha}^{\prime}$, which is also Int GL(V) That is, $\varphi$ stabilizes Int GL(V). Consequently, there is an $S \in G L(V)$ such that for all $g \in \mathrm{GL}(V)$ either

(i) $\varphi($ Int $g)=$ Int $S^{-1} g S$, or

(ii) $\phi($ Int $g)=$ Int $S^{-1} g^{-1} \mathrm{~S}$.

For any $g \in \mathrm{GL}(\mathrm{V})$, Int $g \approx g^{\prime} \otimes^{\prime} g^{-1}$, where $\approx$ denotes equivalence of representations, and for any $g \in \mathrm{U}(\mathrm{V}, \psi)$, the unitary group of some hermitian form $\psi$ in $V$,

$$
' g^{-1} \approx \bar{g} \text {. }
$$

Consequently, for any $\gamma \in \Gamma_{\alpha}^{\prime}$ and $\sigma \in \mathrm{Gal}(\mathrm{F} / \mathrm{E})$,

$$
{ }^{\sigma} \gamma \otimes{ }^{o}(\bar{\gamma})={ }^{o}(\gamma \otimes \bar{\gamma})=\varphi(\text { Int } \gamma) \approx\left\{\begin{array}{l}
\text { Int } \gamma=\gamma \otimes \bar{\gamma}, \\
\text { Int } \bar{\gamma}=\bar{\gamma} \otimes \gamma .
\end{array}\right.
$$

It follows at once that

$$
{ }^{\sigma} \gamma \approx \begin{cases}\lambda(\gamma) \gamma & \text { in Case (i) } \\ \lambda(\gamma) \bar{\gamma} & \text { in Case (ii) }\end{cases}
$$

for all $\gamma \in \Gamma_{\alpha}^{\prime}$ where $\lambda(\gamma) \in \mathbf{C}$. Composing $\sigma$ with complex conjugation, one can assume that we are in Case (i). Then there is an element $S \in G L(V)$ such that for ail $\gamma \in \Gamma_{\alpha}^{\prime}$,

$$
{ }^{\circ} \gamma=S^{-1} \gamma \cdot \lambda(\gamma) S
$$

From ${ }^{\sigma}\left(\gamma_{1} \gamma_{2}\right)={ }^{\sigma} \gamma_{1} \cdot{ }^{\sigma} \gamma_{2}=S^{-1} \gamma_{1} \gamma_{2} \lambda\left(\gamma_{1}\right) \lambda\left(\gamma_{2}\right) S$, we infer that $\lambda: \Gamma_{\alpha}^{\prime} \rightarrow \mathbf{C}$ is a multiplicative homomorphism. For any $\gamma$ such that $\gamma-1$ has rank $1,{ }^{\sigma} \gamma$ has the same property and therefore $\lambda(\gamma)=1$ if $\operatorname{dim} V>2$. Since $\Gamma_{\alpha}$ is generated by pseudo-reflections ((10.19.4), (1 1.2.1)), $\lambda\left(\Gamma_{\alpha}^{\prime}\right)=1$ and ${ }^{\sigma} \operatorname{Tr} \gamma=\operatorname{Tr} \gamma$ for all $\gamma \in \Gamma_{\alpha}^{\prime}$, i.c. $\sigma=1$. This proves $(12 \cdot 5 \cdot 2)$.

We close this scction with a description of the field $\mathbf{E}=\mathbf{Q}\left[\operatorname{Tr}\right.$ Ad $\left.\Gamma_{\alpha}^{\prime \prime}\right]$ in the case $\mathrm{N}=4$, with $\alpha$ satisfying $o<\mu_{\mathrm{s}}<\mathrm{I}$ for all $s \in \mathrm{S}, \Sigma_{\mu_{\mathrm{s}}}=2$, and condition (INT). In this case, set $k_{i}=\left(\mathrm{t}-\mu_{i}-\mu_{j}\right)^{-1}, \mathrm{I} \leq i \neq j \leq 3$. Assume $k_{i j}>0$ (cf. $\S$ I $4 \cdot 3$ ). 
Proposition (12.5 5 ).

$$
\mathrm{E}=\mathbf{Q}\left[\cos ^{2} \frac{\pi}{k_{12}}, \cos ^{2} \frac{\pi}{k_{23}}, \cos ^{2} \frac{\pi}{k_{31}}, \cos \frac{\pi}{k_{12}} \cos \frac{\pi}{k_{23}} \cos \frac{\pi}{k_{31}}\right] .
$$

Proof. - We have $\Gamma_{\alpha} \subset \operatorname{PU}(1,1)$. Thus $\operatorname{Ad} \Gamma_{\alpha}^{\prime}=\operatorname{Ad} \Gamma_{\alpha} \subset \operatorname{SO}(2,1, \mathbf{R})$. Let $\Delta=\Delta\left(k_{12}, k_{23}, k_{31}\right)$ denote the group generated by reflections in the sides of the geodesic triangle in the real hyperbolic 2-space $\mathbf{R} h^{2}$ whose vertices are fixed by the monodromy transformations $\gamma_{12}, \gamma_{23}, \gamma_{31}$ (cf. (12.3) and (12.4.1)), and let $\Delta_{0}$ denote the subgroup of orientation preserving elements in $\Delta$. Then $\operatorname{Ad} \Gamma_{\alpha} \subset \Delta_{0}, \operatorname{card}\left(\Delta / \Delta_{0}\right)=2$, and $\operatorname{Ad} \Gamma_{\alpha}$ is of finite index in $\Delta$.

Let $\langle u, v\rangle$ denote the Killing form on the Lie algebra $\mathscr{L}$ of $\operatorname{PGL}_{2}(\mathbf{C})$, and let $e_{i}$ be an element in $\mathscr{L}$ with $\left\langle e_{i}, e_{i}\right\rangle=\mathrm{I}$ such that (in the projective model of $\mathbf{R} h^{2}$ ) $e_{i}^{\perp}$ contains a side of the above geodesic triangle $(i=1,2,3)$, and $\left\langle e_{i}, e_{i}\right\rangle=-\cos \frac{\pi}{k_{i j}}$ if $i \neq j$. Let $e_{i}^{*}$ denote the element of the dual space of $\mathscr{L}$ such that $e_{i}^{*}(v)=\left\langle v, e_{i}\right\rangle$ for all $v \in \mathscr{L}(i=\mathrm{I}, 2,3)$. Then the three generating reflections of $\Delta$ have the form $s_{i}=1-2 e_{i} \otimes e_{i}^{*}(i=1,2,3)$ and

where

$$
\begin{aligned}
& s_{i_{1}} s_{i_{2}} \ldots s_{i_{r}}=\prod_{j=1}^{r}\left(\mathrm{I}-2 e_{i_{j}} \otimes e_{i_{j}}^{*}\right)=\sum_{k=0}^{r}(-2)^{k} \prod_{\substack{\alpha=1 \\
j_{1}<j_{2} \ldots<j_{k}}}^{k} e_{i_{j_{\alpha}}} \otimes e_{i_{j_{\alpha}}}^{*}, \\
& \operatorname{Tr} s_{i_{1}} \ldots s_{i_{r}}=\sum_{k=0}^{r}(-2)^{k} \prod_{\alpha=1}^{k} c_{i_{j_{\alpha}} i_{j_{\alpha+1}}, \quad j_{k+1}=j_{1}} \\
& c_{i j}=\left\{\begin{array}{cl}
-\cos \frac{\pi}{k_{i j}}, & \mathrm{I} \leq i \neq j \leq 3, \\
\mathrm{I}, & i=j .
\end{array}\right.
\end{aligned}
$$

From this it is clear that $\mathbf{Q}[\operatorname{Tr} \Delta]$ is the ficld $E^{\prime}$ on the right side of (12.5.5). Suppose now $\sigma: \mathbf{E}^{\prime} \rightarrow \mathbf{C}$ is a monomorphism fixing each element of $\mathbf{E}$. By the argument used in (12.2.I) (ii) for any $y \in \Delta$ and $x \in \operatorname{Ad} \Gamma_{\alpha}, y^{-1} \sigma_{y}$ is in the centralizer of $\operatorname{SO}(2,1, \mathbf{R})$. Hence $y^{-1} \sigma_{y}= \pm \mathrm{I}$ and ${ }^{\circ} y= \pm y$. It follows that ${ }^{\circ} \operatorname{det} y= \pm \operatorname{det} y$. But $y \in \mathrm{O}(2,1)$ has determinant $\pm \mathrm{I}$ and this value is fixed by $\sigma$. Therefore ${ }^{\sigma} y=y$ and ${ }^{\circ} \operatorname{Tr} y=\operatorname{Tr} y$ i.e. $\sigma$ fixes each element of $E$. Consequently, $E=E^{\prime}$.

(12.6) Assume card $S \geq 4$. Let $\left\{\mu_{s} \mid s \in S\right\}$ be a family of numbers $0<\mu_{s}<$ I satisfying condition (INT). By Lemma (3.12), $\mu_{s}$ is a rational number for each $s$. By Theorem (II.4), the group $\Gamma_{\alpha}$ is a discrete subgroup of finite covolume in $\operatorname{PU}\left(\mathrm{V}, \psi_{0}\right)$ where $\mathrm{V}=\mathrm{H}^{1}\left(\mathrm{P}_{o}, \mathrm{~L}_{o}\right)$. Set $\mathrm{E}=\mathbf{Q}\left[\operatorname{Tr} \operatorname{Ad} \Gamma_{\alpha}\right], \mathbf{F}=\mathbf{Q}(\sqrt[d]{\mathrm{I}})$ as in (12.1).

Proposition (12.6.1). $-\Gamma_{\alpha}$ is arithmetic in $\mathrm{PU}(\mathrm{V})$ if and only if for each $\sigma \in \mathrm{Gal} F$ whose restriction on $\mathrm{E}$ is not the identity, ${ }^{\sigma} \psi_{0}$ is a definite Hermitian form on ${ }^{\circ} \mathrm{V}$.

Proof. - Let $\mathrm{G}=\mathrm{PU}\left(\mathrm{V}, \psi_{0}\right)$. Inasmuch as the complexification of $\mathrm{G}$ is the simple group PGL(V), (12.6. I) is essentially a restatement of the arithmeticity criterion (12.2.3). 
Proposition (12.6.2). - Assume card S $>$ 4. Then $\Gamma_{\alpha}$ is arithmetic in $\mathrm{PU}(\mathrm{V})$ if and only if for each $\sigma \in \mathrm{Gal} \mathrm{F}$ with $\sigma \neq$ identity or complex conjugation, ${ }^{\circ} \psi_{0}$ is a definite Hermition form on ${ }^{\circ} \mathrm{V}$.

Proof. - This follows at once from (12.6.1) and (12.5.2).

Proposition (12.6.3). - Assume card S $=4$ and $\mu$ satisfies condition (INT). Set

$$
\delta=\operatorname{det}\left(\left\langle e_{i}, e_{j}\right\rangle\right)=\operatorname{det}\left(\begin{array}{ccc}
\mathrm{I} & c_{12} & c_{31} \\
c_{12} & \mathrm{I} & c_{23} \\
c_{31} & c_{23} & \mathrm{I}
\end{array}\right)
$$

where $c_{i j}=-\cos \frac{\pi}{k_{i j}}, \mathrm{I} \leq i, j \leq 3 . \quad$ Then $\Gamma_{\alpha}$ is non-arithmetic in $\mathrm{PU}(\mathrm{V})$ if and only if ${ }^{\circ} \delta<\mathrm{o}$ for some $\sigma \in \mathrm{Gal} \mathrm{E}$ with $\sigma \neq \mathrm{I}$ on $\mathrm{E}$.

Proof. - Wc have $\delta=1-c_{12}^{2}-c_{23}^{2}-c_{31}^{2}-2 c_{12} c_{23} c_{31}$, and thus $\delta \in \mathrm{E}$. Moreover, ${ }^{\circ} \delta>0$ implics that the matrix ${ }^{\circ}\left\langle e_{i}, e_{j}\right\rangle$ is positive definite since all its principal minors are positive. From this (12.6.3) follows.

Gritcrion (12.6.3) applies to the index 2 orientation preserving subgroup of a group gencrated by reflections in the sides of a geodesic triangle in the real hyperbolic 2-space, a so-called " triangle group ", cf. § I 4.3. One can deduce from (12.6.3) that at most a finite number of triangle groups are arithmetic. A complete list of these groups was given by $\mathrm{K}$. Takeuchi in [2I].

Proposition (12.7). - For any $b \in \mathbf{Q}$ let $\langle b\rangle$ denote the fractional part of $b$ i.e. $0 \leq\langle b\rangle\langle\mathbf{I}$ and $b-\langle b\rangle \in \mathbf{Z}$. Let $\mu=\left\{\mu_{s} \mid s \in \mathrm{S}\right\}$ satisfy condition (INT) of (3. I ) and let $d$ denote the least common denominator of $\mu$. Then $\Gamma_{\alpha}$ is an arithmetic lattice in $\mathrm{PU}(\mathrm{V})$ if and only if (12.7.1) for each integer A relatioely prime to $d$ with $\mathrm{I}<\mathrm{A}<d-\mathrm{I}$,

$$
\sum\left\langle A \mu_{s}\right\rangle=1 \text { or card } S-1 \text {. }
$$

Proof. - Let $\mathrm{A}$ be an integer relatively prime to $d$ and let $\varepsilon$ be a primitive root of unity of $\mathbf{F}=\mathbf{Q}(\sqrt[d]{\mathrm{I}})$. Then $\sigma_{\mathrm{A}}: \varepsilon \rightarrow \varepsilon^{\mathrm{A}}$ is an automorphism of $\mathbf{F}$ which is nontrivial on $\mathbf{F} \cap \mathbf{R}$ if and only if $A \neq \equiv \mathrm{I}(\bmod d)$. The automorphism $\sigma_{\mathrm{A}}$ sends the local system with monodromy $\alpha$ to the local system $\left\{\exp 2 \pi i\left\langle\mathrm{~A} \mu_{\mathbf{a}}\right\rangle \mid s \in \mathrm{S}\right\}$. By Corollary (2.21), the hermitian form ${ }^{\sigma_{\Lambda}} \psi_{0}$ has signature

$$
\left(\sum_{s}\left\langle A \mu_{s}\right\rangle-\mathrm{I}, \sum_{s}\left(\mathrm{I}-\left\langle A \mu_{s}\right\rangle\right)-\mathrm{I}\right) \text {. }
$$

The proposition now follows from (12.6.2) and (12.5.2). 
(12.8) Let $\Gamma$ be a subgroup of $\mathrm{GL}(n, \mathbf{Z})$ and let $\mathrm{G}$ denote the Zariski closure of $\Gamma$ in $\mathrm{GL}(n)$. Then $\mathrm{G}$ is an algebraic group defined over $\mathbf{Q}$ with the property

(12.8.1)

Any $\mathbf{Q}$-character $\chi$ of the connected component $G^{c}$ of $I$ in $G$ is trivial.

(For $\chi\left(\Gamma \cap \mathrm{G}^{c}\right)$ is Zariski-dense in the connected subgroup $\chi\left(\mathrm{G}^{c}(\mathbf{C})\right.$ of $\mathbf{C}^{*}$, and it is finite because it has bounded denominators; hencc $\chi\left(\mathrm{G}^{c}\right)=(\mathbf{I})$.)

By a well-known theorem of Borel-Harish Chandra, $\mathrm{G}(\mathbf{R}) / \mathrm{G}(\mathbf{R}) \cap \mathrm{GL}(n, \mathbf{Z})$ has finite Haar volume [3]. Consequently $\Gamma$ is an arithmetic subgroup in $G(R)$ if and only if $\Gamma$ is commensurable with $\mathrm{G}(\mathbf{R}) \cap \mathrm{GL}(n, \mathbf{Z})$ or, equivalently, $\Gamma$ is of finite covolume in $G(\mathbf{R})$.

(12.9) We consider now an algebraic family $X$ defined over $\boldsymbol{Q}$ of curves of the type described in (2.23). We describe an example.

Let $\mu, \alpha, \mathrm{M}$ be as above, let $\mu_{s}=\frac{n_{s}}{d}$ with $d$ the least common denominator for $\left\{\mu_{s} \mid s \in \mathrm{S}\right\}$. Fix $a, b, c \in \mathrm{S}$. Then for each $m \in \mathbf{M}$, there is a unique isomorphism $\mathbf{P} \rightarrow \mathbf{P}^{1}$ mapping $m(a), m(b), m(c)$ respectively to o, $1, \infty$. Let $u: \mathbf{P} \times \mathbf{M} \times \mathbf{P}^{\mathbf{1}}$ be the resulting map. Define

(12.9.1)

$$
\mathrm{X}=\left\{(v, u, m) \in \mathrm{P} \times \mathrm{P} \times \mathrm{M} \mid v^{-d}=\prod_{s \neq c}(u-m(s))^{n_{s}}\right\} .
$$

Let $\pi_{2}, \pi_{3}$ denote the projections of $\mathrm{X}$ onto the second and third factors respectively. Set $\mathrm{X}_{m}=\pi_{3}^{-1}(m)$ and denote by $\pi_{m}$ the restriction of $\pi_{2}$ to $\mathrm{X}_{m}$ for any $m \in \mathrm{M}$.

More generally, let $\pi: \mathrm{X} \rightarrow \mathrm{M}$ be a fiber bundle over $\mathrm{M}$ (i.c., a topological product over small open sets of $M$ ) satisfying

(12.9.2) For each $m \in \mathbf{M}, \pi^{-1}(m)$ is an irreducible abelian cover of $\mathbf{P}$, with covering group $\mathrm{G}$, ramified only at $m(\mathbf{S})$ of orders dividing $d$.

Set $\mathrm{X}_{m}=\pi^{-1}(m)$ for any $m \in \mathrm{M}$. Then $\left\{\mathrm{H}^{1}\left(\mathrm{X}_{m}, \mathbf{Z}\right) \mid m \in \mathrm{M}\right\}$ is a local system on $\mathrm{M}$

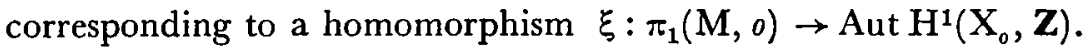

Set $\Gamma=\xi\left(\pi_{1}(\mathrm{M}, o)\right)$; we call $\Gamma$ the $\mathrm{H}^{1}$-monodromy group of the fibration. Moreover the Galois group $\mathscr{G}$ acts fiber by fiber on $\mathrm{X}$ and commutes with horizontal transport of $\mathrm{H}^{1}\left(\mathrm{X}_{m}, \mathbf{Z}\right)$. Consequently, $\mathscr{G}$ commutes elementwise with $\Gamma$. We thus obtain a direct sum decomposition

$(\mathbf{r 2} \cdot \mathbf{9} \cdot \mathbf{3})$

$$
\mathrm{H}^{1}\left(\mathrm{X}_{0}, \mathbf{C}\right)=\bigoplus_{\chi} \mathrm{H}^{1}\left(\mathrm{X}_{o}, \mathbf{C}\right)_{\chi}
$$

where $\chi$ ranges over $\hat{\mathscr{G}}$, the set of characters of $\mathscr{G}$. The group $\mathscr{G}$ is a quotient of $(\mathbf{Z} / d)^{\S} /(\mathbf{Z} / d)$. In the case of (12.9.1), $\mathscr{G}=\mathbf{Z} / d$.

Let $g_{s} \in \mathscr{G}$ be the natural generator of the inertia (= decomposition) group 
at $s$ (cf. (2.23)). Then there is a unique $\chi \in \hat{\mathscr{G}}$ with $\chi\left(g_{s}\right)=\alpha_{s}$ for each $s \in \mathrm{S}$ and by $(2.23 \cdot 1)$

$$
\mathrm{H}^{1}\left(\mathrm{X}_{m}, \mathbf{C}\right)_{\chi}=\mathrm{H}^{1}\left(\mathbf{P}_{m}, \mathrm{~L}_{\chi}\right) \text { for each } m \in \mathrm{M} \text {. }
$$

The vector space $\mathrm{H}^{1}\left(\mathrm{P}_{m}, \mathrm{~L}_{x}\right)$ is defined over the field $\mathbf{Q}(\sqrt[d]{\mathrm{I}})$ and even over its ring of integers by $(12.1 \cdot 3)$.

(12.9.4) Set $F=\mathbf{Q}(\sqrt[d]{\mathrm{I}}), \mathrm{V}=\mathrm{H}^{1}\left(\mathrm{P}_{0}, \mathrm{~L}_{x}\right), \mathrm{W}=\mathrm{V} \otimes_{\mathbf{Q}} \mathrm{F}=\bigoplus_{\sigma \in \mathrm{Gal} F}{ }^{\circ} \mathrm{V}$ where $e_{\mathbf{o}}$ is a primitive idempotent in $\mathrm{C} \otimes_{\mathrm{Q}} \mathrm{F},{ }^{\circ} \mathrm{V}=e_{\sigma} \mathrm{W}$, and $(\mathrm{I} \otimes \tau) e_{\sigma}=e_{\tau \sigma}$ for all $\sigma, \tau \in \mathrm{Gal} \mathrm{F}$. The space $W$ is defined over $\mathbf{Q}$ and we have

$$
W(\mathbf{Q}) \approx \bigoplus_{\sigma \in G \text { al F }} H^{1}\left(P_{o}, L_{\sigma_{x}}(F)\right)
$$

(12.9.5) Set $W(Z)=\bigoplus_{\sigma \in G a l F} H^{1}\left(P_{o}, L_{o_{x}}(\mathcal{O})\right)$ where $\mathcal{O}$ denotes the ring of integers of $F$. The monodromy group $\Gamma$ of the fibration stabilizes $\mathrm{H}^{1}\left(\mathrm{P}_{0}, \mathrm{~L}_{x}(\mathcal{O})\right)$ for each character $\chi \in \hat{\mathscr{G}}$ and in particular stabilizes $\mathrm{W}(\mathbf{Z})$.

Lemma (12.10). - Let $\pi: \mathrm{X} \rightarrow \mathrm{M}$ be an algebraic fiber bundle satisfying (12.9.2) and with non-trivial ramification for each $s \in \mathrm{S}$. Let $\Gamma$ denote the $\mathrm{H}^{1}$-monodromy group of the fibering, let $\mathrm{G}$ be the Zariski-closure of $\Gamma$ in Aut $\mathrm{H}^{\mathbf{1}}\left(\mathrm{X}_{0}, \mathbf{C}\right)$. If $\Gamma$ is of finite covolume in $\mathrm{G}(\mathbf{R})$, then in the euclidean topology $\Gamma_{\chi}$, the projection of $\Gamma$ on Aut $\mathrm{V}$ is dense in $\mathrm{U}\left(\mathrm{V}, \psi_{0}\right)$, where $\chi$ and $\mathrm{V}$ are as in (12.9.4), $\psi_{\mathcal{O}}$ is as in (12.1.1 ), provided there is a $\sigma \in \mathrm{Gal} \mathbf{Q}(\sqrt[d]{1})$ with ${ }^{\circ} \psi_{\mathcal{O}}$ indefinite and $\sigma \neq \pm \mathrm{I}$.

Proof. - Suppose that $\mathrm{G}(\mathbf{R}) / \Gamma$ has finite Haar volume. Then defining the integral structure on $\mathrm{H}^{1}\left(\mathrm{X}_{0}, \mathbf{C}\right)$ as $\mathrm{H}^{1}\left(\mathrm{X}_{0}, \mathbf{Z}\right)$, we infer that $\Gamma$ is commensurable with $\mathrm{G}(\mathbf{Z})=\mathrm{G} \cap$ Aut $\mathrm{H}^{1}\left(\mathrm{X}_{0}, \mathbf{Z}\right)$ by (12.8). Inasmuch as $\mathrm{W}$ is a $\Gamma$-stable subspace defined over $\mathbf{Q}$, it is also G-stable and the restriction of $G(\mathbf{Z})$ to $W$ is an arithmetic subgroup. Consequently $\Gamma_{\mathrm{W}}$, the restriction of $\Gamma$ to $\mathrm{W}$, is arithmetic.

By (12.1.3), $\Gamma_{\chi} \subset \mathrm{U}\left(\mathrm{V}, \psi_{\mathcal{O}}\right)$, and indeed the Zariski-closure of $\Gamma_{x}$ contains $U(\mathrm{~V})$ and even SL(V) - this last fact can be verified by computing the Lie algebras of the Zariski-closures of $\left\{\gamma^{n} ; n \in \mathbf{Z}\right\}$ where $\gamma$ ranges over the pseudo-reflections of $\Gamma_{x}$.

Set $\mathbf{F}=\mathbf{Q}(\sqrt[d]{\mathrm{I}})$. We can regard $\mathrm{U}\left(\mathrm{V}, \psi_{\mathcal{O}}\right)$ as the group of $\mathbf{R}$-rational points of an algebraic group $U$ defined over $F \cap \mathbf{R}$; namely as the subgroup $\left\{(x, \bar{x}) \mid x \in \mathrm{U}\left(\mathrm{V}, \psi_{0}\right)\right\}$ of $\operatorname{Restr}_{\mathbf{C} \mid \mathbf{R}} \mathrm{GL}(\mathrm{V})$. The subgroup $\Gamma_{x}$ then becomes a subgroup of Aut $\mathrm{H}^{1}\left(\mathrm{P}_{o}, \mathrm{~L}_{x}(\mathcal{O})\right) \times$ Aut $^{1}\left(\mathrm{P}_{o}, \mathrm{~L} \sigma_{x}(\mathcal{O})\right)$ and is thus commensurable with $\mathrm{U}(\mathcal{O} \cap \mathbf{R})$. By hypothesis, the group $\mathrm{U}\left({ }^{\circ} \mathrm{V},{ }^{\circ} \psi_{0}\right)={ }^{\circ} \mathrm{U}(\mathbf{R})$ is not compact because the hermitian form ${ }^{\circ} \psi_{\mathcal{O}}$ is indefinite. It remains to explain why $U(\mathcal{O} \cap \mathbf{R})$ is topologically dense in $U(\mathbf{R})$ if $\mathbf{I} \neq \sigma \in \operatorname{Gal}(\mathbf{F} \cap \mathbf{R} / \mathbf{Q})$. This assertion is an immediate consequence of the weak approximation theorem for algebraic groups over an algebraic number field, which applies here since ${ }^{\circ} \mathrm{U}(\mathbf{R})$ is not compact (cf. [I I], p. 192). 
Theorem (12.11). - Let $\mathrm{X}$ be the algebraic family (12.9. I) and let $\Gamma$ be the $\mathrm{H}^{1}$-monodromy group of the fibering. Assume that $\mu$ satisfies condition (INT) of (3.1 I). If $\Gamma$ is of finite covolume in its Zariski-closure $\mathrm{G}(\mathbf{R})$ in Aut $^{1} \mathrm{H}^{1}\left(\mathrm{X}_{0}, \mathbf{R}\right)$, then $\Gamma_{x}$ is an arithmetic lattice in $\mathrm{U}\left(\mathrm{V}, \psi_{\mathcal{O}}\right)$.

Proof. - By Theorem (10.9.1), $\Gamma_{x}$ is discrete in $U\left(V, \psi_{0}\right)$. It follows at once from the Lemma (12.10) that for all $\sigma \in \operatorname{Gal}(\mathbf{Q}(\sqrt[d]{\mathrm{I}}) / \mathbf{Q})$ with $\sigma \neq \pm \mathrm{r},{ }^{\circ} \mathrm{U}\left(\mathrm{V}, \psi_{0}\right)$ is compact. It follows from (12.2) that $\Gamma_{x}$ is arithmetic in $\mathrm{U}\left(\mathrm{V}, \psi_{0}\right)$.

(12. 12) The forcgoing results show that $\Gamma_{x}$ is arithmetic in $U\left(V, \psi_{0}\right)$ if and only if $\Gamma_{w}$ is of finite covolume in its Zariski-closure in Aut W(R).

\section{Elliptic and Euclidean Cases}

The investigation of the map $\widetilde{w}_{\mu}: \widetilde{Q}_{s t} \rightarrow B(\alpha)_{0}$ can be generalized to obtain a class of elliptic and euclidean groups which generalize the stabilizers in $\Gamma_{\alpha}$ of points in $\mathrm{B}(\alpha)_{0}^{+}$and on its boundary. We shall merely sketch the method.

(13. r) Elliptic Case.

Let $\mu_{2}=\left(\mu_{s}\right)_{i \in s_{2}}$ satisfy

a)

$$
\mu_{0}>0 \text {, }
$$

b)

$$
\sum_{0, \mathrm{~s}_{2}} \mu_{3}<\mathrm{I} \text {. }
$$

Augment $S_{1}$ by adjoining an additional element $c$ and set $S=S_{1} \cup\{c\}$. Set $\mu_{e}=2-\sum_{i \in B_{2}} \mu_{\theta}, \mu=\left(\mu_{s}\right)_{s \in S}$. Fix a local system $\mathrm{L}$ on $\mathbf{P}-\mathrm{S}$ with monodromy $\alpha$ ( $\alpha=\exp 2 \pi i \mu$. By (2.2I) (in which $\mu_{0}$ must be replaced by $\mu_{c}-1$ ) the intersection form (, ) is negative definite. Fix $a \in \mathrm{S}_{1}$, take as moduli space $M$ the set of injectivc $y \in \mathbf{P}^{\mathbf{s}}$ with $y(a)=0, y(c)=\infty$ and set

$$
\mathrm{M}_{\mathrm{at}}=\left\{y \in \mathbf{P}^{8} \mid y(c)=\infty, y(a)=0, y\left(\mathrm{~S}_{1}\right) \subset \mathbf{P}-\infty\right\} .
$$

On the family of punctured lines $\mathbf{P}_{\mathbf{k} \mathrm{gt}_{\mathrm{t}}}$ we extend L trivially on each $\mathbf{R}^{+}$near $\infty$ and by monodromy $a$ along each $\mathrm{P}_{y}, y \in \mathrm{M}_{\mathrm{st}}$. Fix $o \in \mathrm{M}$. Let $\widetilde{\mathrm{M}}$ denote the covering of $\mathrm{M}$ corresponding to the monodromy of $\mathrm{H}^{1}\left(\mathrm{P}_{0}, \mathrm{~L}_{0}\right)$, and let $\widetilde{\mathrm{M}}_{\mathrm{at}}$ denote completion of the spread $\widetilde{M} \rightarrow M$ over $M_{\Delta t}$. The section

$$
y \rightarrow w_{\nu}=\prod_{:=e}(z-y(s))^{-\mu_{s}} d z . e
$$

gives a map

$$
\widetilde{w}_{\mu}: \widetilde{\mathrm{M}} \rightarrow \mathrm{H}^{1}\left(\mathrm{P}_{0}, \mathrm{~L}_{0}\right) \text {. }
$$

This map is homogeneous of degree $\mathrm{I}-\sum_{0 \neq c} \mu_{\text {, }}$ relative to the action of the multiplicative group $\mathbf{G}_{m}(y+\lambda y)$ on $\mathbf{M}_{s t}$. By (3.9) the projectivized map is etale on $\mathbf{M}$. Hence $\widetilde{w}_{\mathfrak{k}}$ is 
itself etale. Apply the reasoning of sections 8, 9 and Io replacing the compactness of $Q_{88 t}$ by the compactness of $M_{s t}$-(zero map of $\left.S_{1}\right) / G_{m}$ together with the homogeneity. We then get

Theorem (13.x.1). - I) $\widetilde{w}_{\mu}$ extends to a map $\tilde{\mathrm{M}}_{\mathrm{at}} \rightarrow \mathrm{H}^{1}\left(\mathrm{P}_{o}, \mathrm{~L}_{o}\right)$.

2) If $\mu_{1}$ satisfies (INT): for all $s \neq t$ in $\mathrm{S}_{1},\left(\mathbf{I}-\mu_{s}-\mu_{t}\right)^{-1} \in \mathbf{Z}$, then the extension of $\widetilde{w}_{\mu}$ is an isomorphism.

Corollary (13.1.2). - If $\mu_{1}$ satisfies (INT), then the monodromy group $\Gamma_{\alpha}$ is a finite subgroup of $\mathrm{U}(\mathrm{N}-2), \mathrm{N}=$ card $\mathrm{S}_{1}$ and $\mathrm{H}^{1}\left(\mathrm{P}_{o}, \mathrm{~L}_{\mathrm{o}}\right) / \Gamma_{\alpha} \approx \mathrm{M}_{\mathrm{Bt}}$.

Remark. - The fact that $\mathrm{H}^{1}\left(\mathrm{P}_{o}, \mathrm{~L}_{o}\right) / \Gamma_{\alpha} \approx \mathbf{G}^{\mathrm{X}-\mathbf{2}}$ comes from the fact that $\Gamma_{\alpha}$ is generated by pseudo-reflections (cf. [4] $(\mathrm{V} \cdot 5 \cdot 3)$ théorème 3 ).

(13.2) Euclidean Case.

Let $\mu_{1}=\left(\mu_{s}\right)_{s \in S_{1}}$ satisfy

a)

$$
\begin{aligned}
& \mu_{s}>0, \\
& \sum_{s \in \mathrm{S}_{1}} \mu_{s}=\mathrm{I} .
\end{aligned}
$$

Augment $S_{1}$ as above, setting $S=S_{1} \cup\{c\}, \mu_{c}=2-\sum_{s \in S_{1}} \mu_{s}=1$, and fix $a \in \mathrm{S}_{\mathbf{1}}$. Define

$$
\mathrm{M}_{\mathrm{st}}=\left\{y \in \mathbf{P}^{\mathrm{S}} \mid y(c)=\infty, y(a)=0, y\left(\mathrm{~S}_{\mathbf{1}}\right) \subset \mathbf{P}-\infty, y\left(\mathrm{~S}_{\mathbf{1}}\right) \neq\{\mathrm{o}\}\right\} .
$$

Set $Q_{\mathrm{st}}=\mathrm{M}_{\mathrm{st}} / \mathbf{G}_{m}$; it is compact. We define $\mathrm{L}$ on $\mathrm{P}_{\mathrm{M}_{\mathrm{st}}}$ and define $w_{y}$ as above. The local system $\mathrm{L}$ has no monodromy at $\infty$. Accordingly, we set $\mathrm{S}^{\prime}=\{c\}$ and work with $\mathrm{H}_{\varphi\left(\mathrm{B}^{\prime}\right)}^{1}\left(\mathrm{P}_{o}, \mathrm{~L}_{o}\right)$, cohomology of $\mathrm{P}-o(\mathrm{~S})$ with support in the family $\varphi\left(\mathrm{S}^{\prime}\right)$ of subsets of $\mathrm{P}-o(\mathrm{~S})$ closed in $\mathrm{P}-\{\infty\})$. By (2.15.2), wy represents a non-zero cohomology class in $\mathrm{H}_{\phi\left(\mathbf{S}^{\prime}\right)}^{1}\left(\mathrm{P}_{n}, \mathrm{~L}_{o}\right)$. For all $y \in \mathrm{P}_{\mathrm{M}}$, one has $\operatorname{Res}_{\infty}\left(w_{y}\right)=\mathrm{I}$, the unit element of the local system $\mathrm{L}$ which is trivial near $\infty$. Inasmuch as

$$
\left\langle\text { cycle around } \infty, w_{y}\right\rangle=\mathrm{I}
$$

for all $y \in \mathrm{P}_{\mathrm{M}}$, the image of the map $\widetilde{w}_{k \mu}: \mathrm{M} \rightarrow \mathrm{H}_{\phi\left(\mathrm{S}^{\prime}\right)}^{1}\left(\mathrm{P}_{0}, \mathrm{~L}_{0}\right)$ lies on an affine hyperplane $\mathrm{H}$. This hyperplane $\mathrm{H}$ on which $w_{y}$ lives is parallel to the homogeneous one given by $\langle$ cycle around $\infty\rangle=$,0 . This vector subspace of $\mathrm{H}_{q\left(\mathrm{~S}^{\prime}\right)}^{1}\left(\mathrm{P}_{o}, \mathrm{~L}_{o}\right)$ can be identified with $\mathrm{H}_{c}^{1}\left(\mathbf{P}-o\left(\mathrm{~S}_{1}\right), \mathrm{L}_{\mathbf{1}}\right)$ where $\mathrm{L}_{1}$ is the local system on $\mathbf{P}-o\left(\mathrm{~S}_{1}\right)$ with monodromy $\mu_{1}$ (or equivalently with $\mathrm{H}^{1}\left(\mathbf{P}-o\left(\mathrm{~S}_{1}\right), \mathrm{L}_{1}\right.$ ) by (2.6)). The intersection form on $H^{1}\left(\mathbf{P}-o\left(S_{1}\right), L_{1}\right)$ is negative definite by $(2.2 \mathrm{I})$.

The map $y \mapsto w_{y}$ is homogeneous of degree o for the $\mathbf{G}_{m}$ action and therefore defines a map $\widetilde{w}_{\mu}: \widetilde{\mathbf{Q}} \rightarrow \mathrm{H}$.

Theorem (13.2.1). - I) $\widetilde{w}_{\mu}$ extends to a map $\widetilde{w}_{\mu}$ of $\widetilde{\mathbf{Q}}_{\text {st }} \rightarrow \mathrm{H}$.

2) If $\mu_{1}$ satisfies (INT), then the extended $\widetilde{w}_{\mu}$ is an isomorphism. 
Corollary (13.2.2). - If $\mu_{1}$ satisfies (INT), then the monodromy group $\Gamma_{\alpha}$ contains a subgroup of finite index $\mathrm{T}$ of translations of the affine space $\mathrm{H}, \mathrm{H} / \mathrm{T}$ is an abelian variety, and

$$
\mathbf{P}_{\mathrm{N}-2}=\mathrm{Q}_{\mathrm{st}} \approx \mathrm{H} / \Gamma_{\alpha}=\text { (abelian variety) } /(\Gamma / \mathrm{T}),
$$

where $\mathrm{N}=$ card $\mathrm{S}$.

\section{Lists of $\mu$ associated to discrete groups}

(14.r) Elliptic and Euclidean lists.

Set $\operatorname{card} S_{e}=N$, assume $\mu_{s}>0$ for all $s \in S_{e}$ and $\sum_{s \in B_{e}} \mu_{s} \leq 1$. For any $s \neq t$ in $\mathrm{S}_{e}$, set

$$
\begin{aligned}
k_{s, t} & =\left(\mathrm{I}-\mu_{t}-\mu_{t}\right)^{-1}, \\
\mathrm{D} & =\left(\mathrm{I}-\sum_{\bullet \in \mathrm{B}_{t}} \mu_{s}\right)^{-1} .
\end{aligned}
$$

We assume (INT): each $k_{a, t} \in \mathbf{Z} \cup \infty$. By a " miracle of small numbers" it will be seen that $\mathrm{D}$ is an integer or $\infty$ (cf. (6.10) and (10.4) (i)).

Summing the $N(N-1) / 2$ equalities

$$
\mu_{s}+\mu_{t}=\mathrm{I}-\frac{\mathrm{I}}{k_{\mathrm{at}}}
$$

gives

$$
(\mathrm{N}-\mathrm{I}) \Sigma_{\mu_{s}}=\frac{\mathrm{N}(\mathrm{N}-\mathrm{I})}{2}-\Sigma \frac{\mathrm{I}}{k_{s t}}
$$

Hence

$$
\sum \frac{\mathrm{I}}{k_{s t}}=\frac{\mathrm{N}(\mathrm{N}-\mathrm{I})}{2}-(\mathrm{N}-\mathrm{I})\left(\mathrm{I}-\frac{\mathrm{I}}{\mathrm{D}}\right)=\frac{(\mathrm{N}-\mathrm{I})(\mathrm{N}-2)}{2}+\frac{\mathrm{N}-\mathrm{I}}{\mathrm{D}} .
$$

Since $k_{s, t} \geq 2,(3)$ implics

$$
1-\frac{\mathrm{I}}{\mathrm{D}} \geq \frac{\mathrm{N}}{4}
$$

Hence $\mathrm{N} \leq 4$ and $\mathrm{N}=4$ only if $\mathrm{D}=\infty, k_{s, t}=2$ for all $s, t$ and $\mu_{8}=\frac{\mathrm{I}}{4}$ for all $s \in \mathrm{S}_{e}$. If $\mathrm{N}=3$, (3) yields

$$
\frac{\mathrm{I}}{\mathrm{D}}=\frac{\mathrm{I}}{2}\left(\sum \frac{\mathrm{I}}{k_{\mathrm{i}, t}}-\mathrm{I}\right)
$$

and solving the system (I) yields

$$
\begin{aligned}
\mu_{s} & =\frac{\mathrm{I}}{2}\left(\left(\mathrm{I}-\frac{\mathrm{I}}{k_{s, t}}\right)+\left(\mathrm{I}-\frac{\mathrm{I}}{k_{s u}}\right)-\left(\mathrm{I}-\frac{\mathrm{I}}{k_{t u}}\right)\right) \\
\text { (14.1.1) } \mu_{s} & =\frac{\mathrm{I}}{2}\left(\mathrm{I}-\sum k_{s, t}^{-1}+2 k_{t u}^{-1}\right)=\frac{\mathrm{I}}{k_{t u}}-\frac{\mathrm{I}}{\mathrm{D}} .
\end{aligned}
$$


(14.1.2) The number D satisfying (4), must be an even integer or $\infty$.

For if a sum of three integer reciprocals exceeds $I$, the excess is an integer reciprocal (cf. $(6$. ro)).

We list all the possibilities:

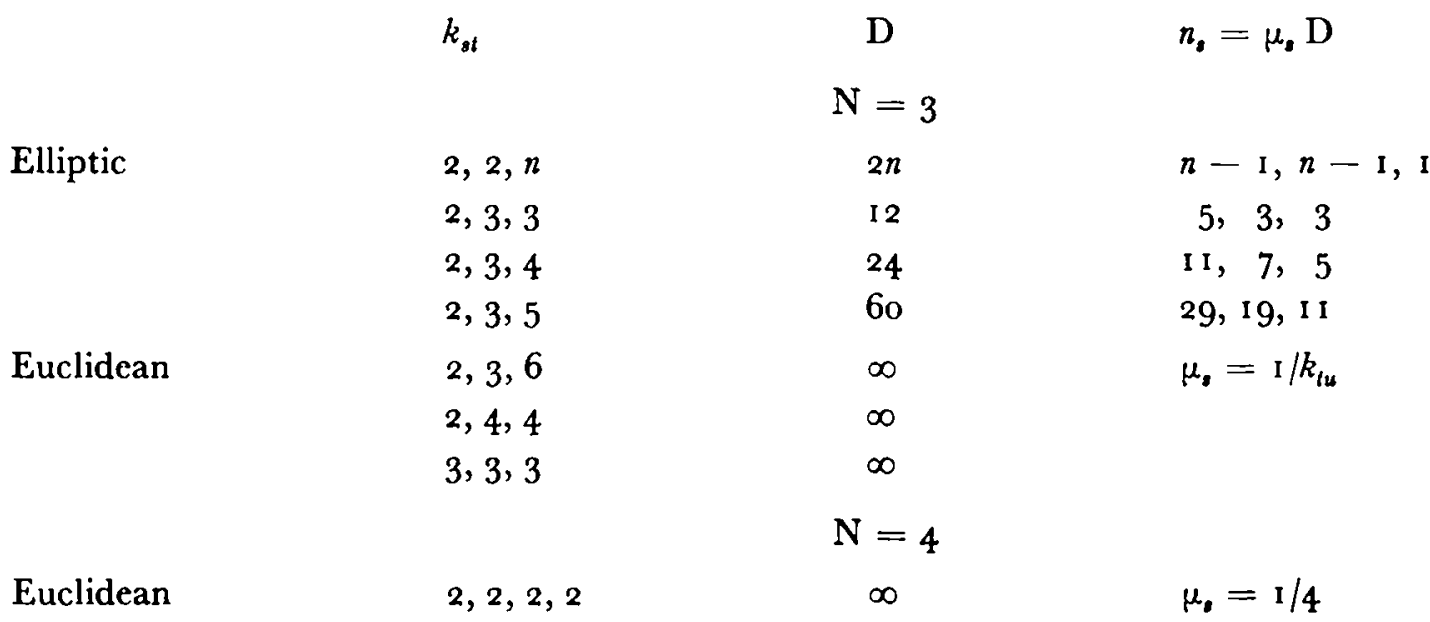

(r4.2) Let $N=$ card $S$ and assume that $N \geq 5$. The foregoing results permit one to infer limitations on the possible $\mu=\left(\mu_{0}\right)_{, \in S}$ satisfying

I) $\mu_{s}>0$ for each $s \in \mathrm{S}$,

2) $\sum_{i \in \mathrm{S}} \mu_{\mathrm{s}}=2$,

3) $k_{a t}=\left(\mathbf{I}-\mu_{\mathrm{s}}-\mu_{t}\right)^{-1} \in \mathbf{Z}$ if $\mu_{\mathrm{s}}+\mu_{t}<\mathrm{I}$.

Case A: for some $s \neq t$ in S, $\mu_{s}+\mu_{t}>1$. By (14.1), applied to the complement of $\{s, t\}$ in $\mathrm{S}$, we have $\mathrm{N}=5$. Set $\mathrm{S}=\{s, t, a, b, c\}$. We define $\mathrm{D}$ by

$$
\mu_{0}+\mu_{t}=\mathrm{I}+\frac{\mathrm{I}}{\mathrm{D}} \text {. }
$$

Then $\mu_{a}+\mu_{b}+\mu_{c}=I-\frac{I}{D}$ and by (I 4.I.I) $\mu_{a}, \mu_{b}, \mu_{c} \leq \frac{I}{2}-\frac{I}{D}$. Choosing $\mu_{a} \geq \mu_{b}$ we find $\mu_{t}+\mu_{u}<\mathrm{I}$ for $u \in\{a, b, c\}$.

(14.2.1) $\mathrm{D}$ is an even integer (by (14.1.2)).

Case B: for some $s, t \in \mathrm{S}, \mu_{\mathrm{s}}+\mu_{t}=1$. The remaining $\mu^{\prime} s$ make up a euclidean $\mu_{1}$ so that $\mathrm{N}=5$ or 6 . If $\mathrm{N}=5, \mathrm{~S}=\{s, t, a, b, c\}$ with $\mu_{u} \leq \frac{\mathrm{I}}{2}$ and $\mu_{t}+\mu_{\mathrm{u}} \leq \mathrm{I}$ for $u \in\{a, b, c\}$ if $\mu_{1} \geq \mu_{t}$. If $\mathrm{N}=6, \mu=\left(\mu_{s}, \mu_{4}, \frac{\mathrm{I}}{4}, \frac{\mathrm{I}}{4}, \frac{\mathrm{I}}{4}, \frac{\mathrm{I}}{4}\right)$. 
Case C: neither A nor $B$ hold. Here we see the $N(N-I) / 2$ equalities $\mu_{s}+\mu_{t}=1-\frac{1}{k_{m}}$ yields

$$
(N-\text { I }) \Sigma_{\mu_{2}} \geq \frac{N(N-I)}{2} \cdot \frac{1}{2} \text {. }
$$

Hence $\mathrm{N} \leq 8$ and $\mathrm{N}=8$ implies $k_{s, t}=2$ for all $s, t \in \mathrm{S}$ i.e. $\mu_{\mathrm{a}}=\frac{1}{4}$ for all $s \in \mathrm{S}$.

In particular

(14.2.2) If $\left(\mu_{s}\right)_{s \in S}$ satisfies 1$\left.\left.), 2\right), 3\right)$, then card $S \leq 8$.

We explain in section 15 how the list for $N=5$ can be gleaned from the thesis of Le Vavasseur. For $\mathrm{N}>5$, the lists are easily obtainable in Case C. Arrange $\mu$ in descending under $\mu_{1} \geq \mu_{2} \geq \ldots \geq \mu_{N}$. Then (cf. (6. Io.I)) $\left(\mu_{1}+\mu_{2}\right), \mu_{3}, \ldots, \mu_{N}$ must be on the list for $\mathrm{N}-\mathrm{I}$ with $\left(\mu_{1}+\mu_{2}\right) \geq 2 \mu_{3}$. Iteration of this criterion leads to the compact quotients $\mathrm{PU}(\mathrm{I}, \mathrm{N}-3) / \Gamma_{\alpha}$ for $\mathrm{N} \geq 5$. As for the non-compact quotients with $\mathrm{N}>5$, by Case $\mathrm{B}$ these are $\left(\mu_{1}, \mu_{2}, \frac{\mathrm{I}}{4}, \frac{\mathrm{I}}{4}, \frac{\mathrm{I}}{4}, \frac{\mathrm{I}}{4}\right)$; only three solutions satisfy condition (INT): $\left(\mu_{1}, \mu_{2}\right)=\left(\frac{1}{2}, \frac{I}{2}\right),\left(\frac{3}{4}, \frac{I}{4}\right),\left(\frac{7}{12}, \frac{5}{12}\right)$.

(14.3) The case $N=4$. Let $\left(\mu_{i}\right)_{1 \leq i \leq 4}$ be a 4 -tuple of real numbers which satisfy $0<\mu_{i}<\mathrm{I}, \Sigma \mu_{i}=2$. If the $\mu^{\prime}$ 's are arranged with $\mu_{1} \leq \mu_{2} \leq \mu_{3} \leq \mu_{4}$, one has $\mu_{1}+\mu_{3} \leq \mu_{2}+\mu_{4}=2-\left(\mu_{1}+\mu_{3}\right)$, hence $\mu_{1}+\mu_{3} \leq \mathrm{I}$. A fortiori, $\mu_{1}+\mu_{2} \leq \mathrm{I}$. If $\mu_{2}+\mu_{3} \leq \mu_{1}+\mu_{4}$, then $\mu_{i}+\mu_{j} \leq 1$ for $i \neq j$ among $\{1,2,3\}$. If not, $\mu_{1}+\mu_{i} \leq \mathrm{I}$ for $i=2,3,4$, hence $\mu_{j}+\mu_{k} \geq \mathrm{I}$ for $j \neq k$ among $\{2,3,4\}$. One goes back and forth between those two cases by the transformation $\mu_{i}^{\prime}=\mathrm{I}-\mu_{i}$, followed by the relabelling $i \mapsto 5-i$. In the first case, if we put $\mu_{1}+\mu_{2}=1-\frac{1}{p}$, $\mu_{1}+\mu_{3}=\mathrm{I}-\frac{\mathrm{I}}{q}, \mu_{2}+\mu_{3}=\mathrm{I}-\frac{\mathrm{I}}{r}$, we get

$(\mathbf{1} \cdot 3 \cdot \mathbf{1})$

$$
\begin{aligned}
& \mu_{1}=\frac{I}{2}\left(I-\frac{I}{p}-\frac{I}{q}+\frac{I}{r}\right), \\
& \mu_{2}=\frac{I}{2}\left(I-\frac{I}{p}+\frac{I}{q}-\frac{I}{r}\right), \\
& \mu_{3}=\frac{I}{2}\left(I+\frac{I}{p}-\frac{I}{q}-\frac{I}{r}\right), \\
& \mu_{4}=\frac{I}{2}\left(I+\frac{I}{p}+\frac{I}{q}+\frac{I}{r}\right) ;
\end{aligned}
$$

these formulas provide a solution of (INT) for each triple of positive integers $[p, q, r]$ satisfying $\frac{\mathrm{I}}{p}+\frac{\mathrm{I}}{q}+\frac{\mathrm{I}}{r} \leq \mathrm{I}, \mathrm{I}<p \leq q \leq r \leq \infty$. The $\mu_{i}^{\prime}=\mathrm{I}-\mu_{i}$ are given by the same formula, with $\mathrm{I} / p, \mathrm{I} / q$ and $\mathrm{I} / r$ replaced by their negatives (see (14.3.2)). All solutions of (INT) are obtained in this way. 
If $\mathrm{L}$ is a rank one local system on $\mathbf{P}^{1}$ minus four points with monodromy $\alpha=\exp (2 \pi i \mu)$, the dual local system has monodromy $\alpha^{-1}=\exp \left(2 \pi i \mu^{\prime}\right)$, with $\mu_{i}^{\prime}=\mathrm{I}-\mu_{i}$. The vector spaces $H^{1}(\mathrm{~L})$ and $\mathrm{H}^{1}(\mathrm{~L})$ are in duality. As they are of dimension 2, this duality provides a canonical isomorphism between $\mathrm{PH}^{1}(\mathrm{~L})$ and $\mathrm{PH}^{1}(\check{\mathrm{L}})$ : to a line in $\mathrm{H}^{\mathbf{1}}(\mathbf{L})$, one associates its orthogonal in $\mathrm{H}^{\mathbf{1}}(\breve{\mathbf{L}})$. On $\mathrm{Q}$, this gives a canonical isomorphism between the flat projective line bundles defined by $\mu$ and $\mu^{\prime}$. Via this isomorphism, the holomorphic sections $w_{\mu}$ and $w_{\mu^{\prime}}$ are identified; for both $w_{\mu}$ and $w_{\mu^{\prime}}$ are of the first kind, and $\left\langle w_{\mu}, w_{\mu^{\prime}}\right\rangle$, given by the integral of the exterior product of two holomorphic differentials is o; cf. (2.18) and the computations in (2.19). In view of this identification, we will limit ourselves to the second case, with $\mu$ 's given in descending order by

$(14 \cdot 3 \cdot 2)$

$$
\begin{aligned}
& \mu_{1}^{\prime}=\frac{\mathrm{I}}{2}\left(\mathrm{I}+\frac{\mathrm{I}}{p}+\frac{\mathrm{I}}{q}-\frac{\mathrm{I}}{r}\right), \\
& \mu_{2}^{\prime}=\frac{\mathrm{I}}{2}\left(\mathrm{I}+\frac{\mathrm{I}}{p}-\frac{\mathrm{I}}{q}+\frac{\mathrm{I}}{r}\right),
\end{aligned}
$$

$$
\begin{aligned}
& \mu_{3}^{\prime}=\frac{1}{2}\left(\mathrm{I}-\frac{\mathrm{I}}{p}+\frac{\mathrm{I}}{q}+\frac{\mathrm{I}}{r}\right), \\
& \mu_{1}^{\prime}=\frac{\mathrm{I}}{2}\left(\mathrm{I}-\frac{\mathrm{I}}{p}-\frac{\mathrm{I}}{q}-\frac{\mathrm{I}}{r}\right),
\end{aligned}
$$

with

$$
\mathrm{I}<\boldsymbol{p} \leq q \leq r \leq \infty, \frac{\mathrm{I}}{p}+\frac{\mathrm{I}}{q}+\frac{\mathrm{I}}{\mathrm{r}}<\mathrm{I} \text {. }
$$

For such a system of $\mu$ 's, $Q_{\text {sot }}$ can be identified with the space of $y:\{1,2,3,4\} \rightarrow \mathbf{P}^{1}$ with $y(\mathrm{I})=0, y(2)=\mathrm{I}, y(3)=\infty$, i.e. (using $x:=y(4)$ as coordinate) with $\mathbf{P}^{1}$. The multivalued map $w$ admits as projective coordinates the integrals (dropping the primes from $\mu^{\prime}$ )

$$
\int z^{-\mu_{1}}(z-1)^{-\mu_{2}}(z-x)^{-\mu_{d}} d z \text {. }
$$

In his cited paper [20], Schwarz proved that the multivalued map $w$ induces a bijection from the upper half plane $\operatorname{Im}(x)>0$ to a geodesic triangle in the hyperbolic ball $\mathrm{B}(\alpha)^{+}$with angles $\pi / p, \pi / q, \pi / r$. It is of interest to deduce this classical result from the theorems proved in this paper.

To begin with, $w$ is étale (Prop. (3.9)) and has the local behaviour described in $\S 9.6$ near 0 , I, and $\infty$.

Next we show that $w$ maps each of the segments $\left.\tau_{1}=\right]-\infty, o\left[, \tau_{2}=\right] 0, \mathrm{I}[$, and $\left.\tau_{3}=\right] \mathrm{I}, \infty\left[\right.$ to circular $\operatorname{arcs}$ in $\mathrm{B}(\alpha)^{+}$. It suffices to prove this for ] $-\infty, o[$, since the segments are permuted by $O \mapsto \mathrm{I} \mapsto \infty \mapsto 0$.

Take $x$ near $]-\infty, o\left[\right.$ and as homogeneous coordinates for $w(x)$ the integrals $\int_{0}^{1}$ and $\int_{1}^{\infty}$ with the principal determination of the integral. In these coordinates, $w(\bar{x})=w(x)^{-}$. Independently of coordinates, we get $w(\bar{x})=\sigma w(x)$ for $\sigma$ an antiholomorphic involution of $\mathbf{P}^{1}$ i.e. a Schwarz reflection. It follows that $w(]-\infty, 0[)$ 
lies on the fixed point set of $\sigma$, a circular arc. Consequently, $w$ induces an injective map of the real axis to the boundary of a triangle in $\mathbf{P}^{1}$ with circular arcs which lic in $\mathbf{B}(\alpha)^{+}$, and the map $w$ has a holomorphic extension to the upper half plane. From the fact that the map $w$ is étale and has image in $\mathrm{B}(\alpha)^{+}$, it follows by a maximum modulus argument that the $w$-image of the closed upper half plane is the closed triangle $\Delta$ lying in $B(\alpha)^{+}$ with the given circular arc boundary. That $w$ is a bijection follows from Theorem (10.18.2) (whose proof simplifies vastly for $\mathrm{N}=4$ ).

We show finally that the circular arcs are geodesic lines in $\mathrm{B}(\alpha)^{+}$. Regarded as a single valued map of $\chi_{a t}, w$ is a $\pi_{1}(Q, o)$-equivariant map. Set $c_{i}=w\left(\tau_{i}\right)$, using the determination of $w$ described above, and let $\sigma_{i}$ denote the reflection in $c_{i}(i=1,2,3)$. Then $\sigma_{i} \sigma_{j}$ is a holomorphic self-map of $\mathbf{P}^{1}$ with $\sigma_{i} \sigma_{j} w$ an analytic continuation of $w$ regarded as a multivalued holomorphic function on $Q$. Indeed if $i \neq j, \sigma_{i} \sigma_{j}$ is the monodromy in $\Gamma_{\mu}$ corresponding to one turn of $x$ around $\partial c_{i} \cap \partial c_{j}$. Consequently $\left\{\sigma_{i} \sigma_{j} ; \mathrm{I} \leq i<j \leq 3\right\}$ generates $\Gamma_{\mu}, \mathrm{B}(\alpha)^{+}=\Gamma_{\mu}\left(\Delta \cup \sigma_{1} \Delta\right)$, and the group generated by $\left\{\sigma_{i} ; i=\mathrm{r}, 2,3\right\}$ is $\Gamma_{\mu} \cup \sigma_{1} \Gamma_{\mu}$. It follows at once that $\sigma_{1} \mathrm{~B}(\alpha)^{+}=\mathrm{B}(\alpha)^{+}$. Hence $\sigma_{1}$ is an isometry of $\mathrm{B}(\alpha)^{+}$. Consequently $c_{1}$ is a geodesic line. Since our arguments persist under permutation of indices, all the sides of $\Delta$ are geodesics.

To sum up,

(14.3.3) The 4-tuples $\mu_{1} \leq \mu_{2} \leq \mu_{3} \leq \mu_{1}$ satisfying $0<\mu_{i}<1$ and $\sum_{i} \mu_{i}=2$ correspond pairwise to triangle groups $[p, q, r]$ with $\mathrm{I}<p \leq q \leq r \leq \infty$, the pairs being related by $\mu_{5-i}^{\prime}=\mathrm{I}-\mu_{i}, i=\mathrm{I}, 2,3,4 . \quad \mu$ and $\mu^{\prime}$ coincide only if $r=\infty$.

(14.3.4) For any $N \geq 4$, the monodromy representations of $\pi_{1}(Q, o)$ corresponding to the pair $\left(\mu, \mu^{\prime}\right)$ are contragredient, but for $\mathrm{N}=4$ these two representations are equivalent via the canonical isomorphism of $\mathrm{PH}^{1}(\mathrm{~L})$ to $\mathrm{PH}^{1}(\breve{\mathrm{L}})$.

(14.3.5) Complex conjugation maps $\mathrm{L}$ to $\breve{\mathrm{L}}$ and induces a semi-linear isomorphism $\kappa$ of $\mathrm{H}^{1}(\breve{\mathrm{L}})$ to $\mathrm{H}^{1}(\check{\mathrm{L}})$ which maps $\mathrm{H}^{0,1}(\mathrm{~L})$ to $\mathrm{H}^{1,0}(\breve{\mathrm{L}})$. Identifying $\mathrm{PH}^{1}(\mathrm{~L})$ to $\mathrm{PH}^{1}(\breve{\mathrm{L}})$ via the canonical map, the map $\boldsymbol{x}$ induces the anti-holomorphic reflection of $\mathbf{P}^{1}$ in the boundary of the ball $\mathrm{B}(\alpha)^{+}$. It is only for $\mathrm{N}=4$ that the complement of the closed ball in $\mathrm{P}^{\mathrm{X}-3}$ is again a ball.

(14.4) We list below all solutions for $\mu$ satisfying condition (INT) where $o<\mu_{\mathrm{a}}<$ I for all $s, \Sigma \mu_{s}=2$, and card $S>4$. Set

$\mathrm{N}=$ card $\mathrm{S}$,

$d=$ lowest common denominator of $\mu=\left(\mu_{s}\right)_{\ell \in 8}$,

$n_{s}=d \mu_{s}$,

NA = non-arithmetic (cf. (12.7.I) for criterion), $\infty$ means that $\mathrm{PU}(\mathrm{I}, \mathrm{N}-3) / \Gamma_{\alpha}$ is not compact.

No entry in the last (resp. next to the last) column indicates compact quotient (resp. arithmetic lattice). 


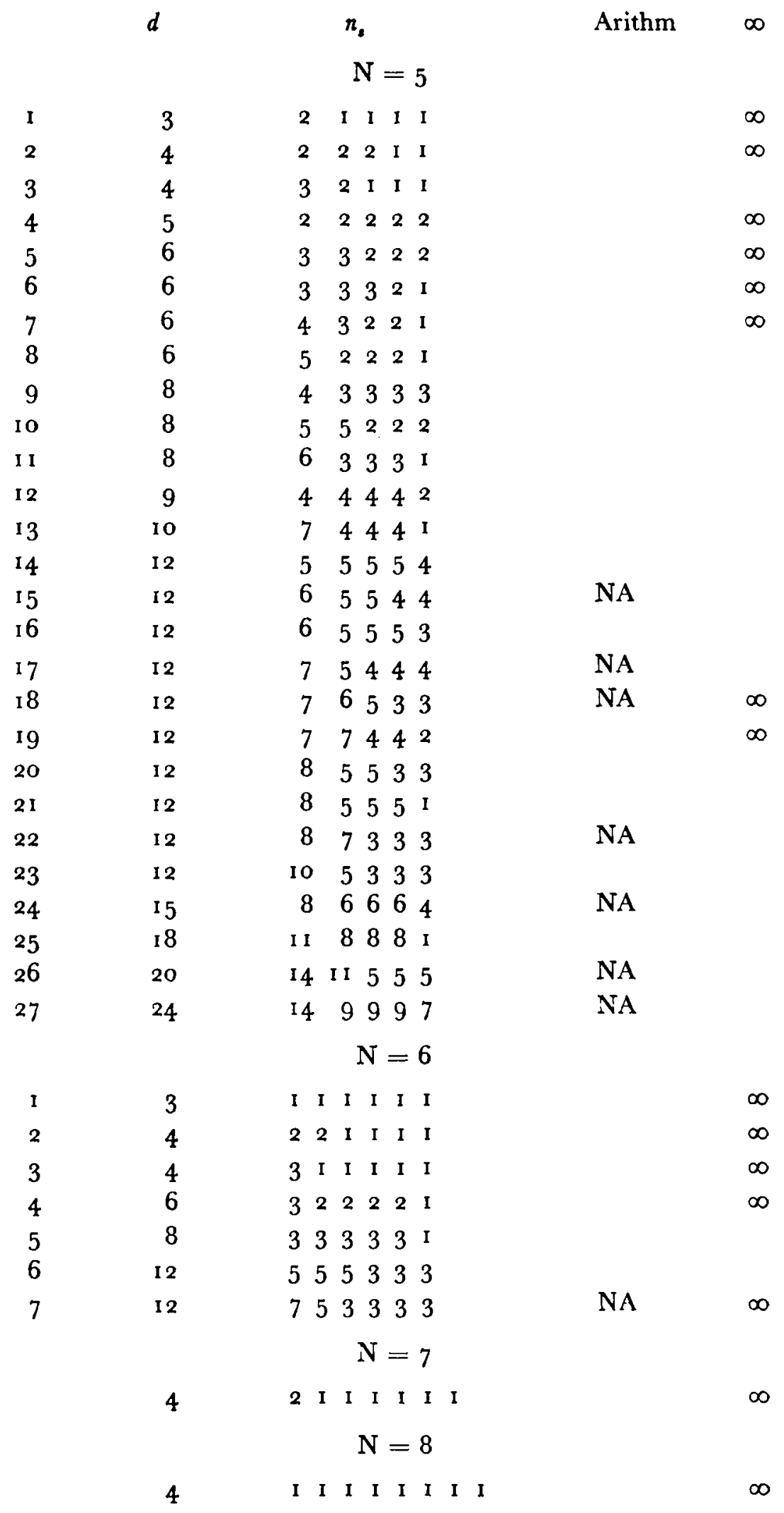




\section{LeVavasseur's list}

In his 1885 paper [ $16 b]$, Picard gave as sufficient condition for the monodromy group $\Gamma$ of the multivalued function

$$
\begin{aligned}
\mathrm{F}(x, y)=\int_{1}^{\infty} u^{\lambda_{0}-1}(u-\mathrm{I})^{\lambda_{\mathrm{s}}-1}(u-x)^{\lambda_{1}-1}(u-y)^{\lambda_{2}-1} d u & \\
& \left(0<\lambda_{i}<\mathrm{I} \text { all } i\right),
\end{aligned}
$$

to be discrete the set of 10 integrality conditions

$$
\text { (r5.x) } \quad\left(\lambda_{i}+\lambda_{j}-\mathrm{I}\right)^{-1} \in \mathbf{Z}^{+} \cup \infty, \quad\left(3-\sum_{j \neq i} \lambda_{i}\right)^{-1} \in \mathbf{Z}^{+}, \quad 0 \leq i \neq j \leq 3 .
$$

In his 1887 note $[16 c]$ Picard asserts without proof that the above Io integers need not be $>o$ but may be negative as well and still $\Gamma$ is discrete. R. Le Vavasseur, in his 1893 dissertation written under Picard's direction found all solutions of

$$
\left(\lambda_{i}+\lambda_{j}-\mathrm{I}\right)^{-1} \in \mathbf{Z} \cup \infty, \quad\left(3-\sum_{j \neq i} \lambda_{j}\right)^{-1} \in \mathbf{Z}, \quad 0 \leq i \neq j \leq 3 .
$$

Setting $\mu_{i}=1-\lambda_{i}(0 \leq i \leq 3)$ and $\mu_{4}=2-\sum_{0}^{3} \mu_{i}$ we can rewrite these 10 conditions as

$$
\begin{aligned}
& \sum_{0}^{4} \mu_{i}=2, \\
& \left(\mu_{i}+\mu_{j}-1\right)^{-1} \in \mathbf{Z} \cup \infty \text { if } 0 \leq i \neq j \leq 4 .
\end{aligned}
$$

In [13], Le Vavasseur lists 102 solutions of [L]. If in addition one imposes the inequalities $0<\mu_{i}<_{1}$ for $0 \leq i \leq 4$, the resulting 5 -tuple solutions, ignoring order, reduce to 27 . These are the 27 listed in (14.3) under $N=5$; that is to say, condition $\left[\mathrm{L}^{\prime}\right]$ :

$$
\begin{aligned}
& 0<\mu_{i}<\mathrm{I} \quad(0 \leq i \leq 4), \\
& \sum_{0}^{4} \mu_{i}=2, \\
& \left(\mathrm{I}-\mu_{i}-\mu_{j}\right)^{-1} \in \mathbf{Z} \cup \infty \quad \text { if } i \neq j,
\end{aligned}
$$

is equivalent to condition (INT) of (3.11). The reason for this striking coincidence is explained by (14.2), Case A. Thus the apparently stronger integrality conditions imposed by Picard in $[16 b]$ and $[16 c]$ are equivalent to our condition (INT).

To complete the historical record we note that Le Vavasseur's condition [L] admits 10 solutions not satisfying $\mathrm{L}^{\prime}$, one of which, $\left(0,0,0, \mathrm{I}-\frac{1}{n}, 1+\frac{1}{n}\right)$, consists 
of an infinite number. The image of the map $\widetilde{w}_{\mu}$ is not a ball for these ro solutions. We illustrate briefly the case of two solutions

and

$$
\begin{aligned}
& \mu=\left(-\frac{2}{3}, \frac{2}{3}, \frac{2}{3}, \frac{2}{3}, \frac{2}{3}\right) \\
& \mu=\left(-\frac{1}{2}, \frac{1}{2}, \frac{1}{2}, \frac{I}{2}, I\right) .
\end{aligned}
$$

(i) Set $\mathrm{S}=\{0,1,2,3,4\}, \mu=\left(-\frac{2}{3}, \frac{2}{3}, \frac{2}{3}, \frac{2}{3}, \frac{2}{3}\right)$. By $(2.21)$ the signature of the hermitian form is $(2, \mathrm{I})$ rather than the usual $(\mathrm{I}, 2)$, and $\int w \wedge \vec{w}>0$ as usual. Consequently $\widetilde{w}_{\mu}: \widetilde{Q}_{s t} \rightarrow P U(V)$ maps $\widetilde{Q}_{s t}$ to the complement of the ball $B(\alpha)_{0}^{+}$. The subset $\widetilde{Q}_{01}$ of $\widetilde{Q}_{8 t}$ corresponding to the two points $x_{0}$ and $x_{1}$ coming together maps to the fixed point set of the monodromy $\gamma_{01}$ of $x_{1}$ around $x_{0}$-which is unipotent since $\mu_{0}+\mu_{1}$ is integral (cf. $(12 \cdot 3 \cdot 2)$ ). Hence $\widetilde{Q}_{01}$ maps to a line tangent to the boundary of the ball at the point fixed by $\gamma_{01}$. It is easy to verify that the monodromy group $\Gamma_{\alpha}$ is a matrix group with coefficients in $\mathbf{Z}(\sqrt[3]{\mathrm{I}})$ and is therefore discrete in $U(2, I)$. However, lattice subgroups of $\mathrm{PU}(\mathrm{V})$ do not operate discontinuously on the complement of the ball, so that the strategy of Section Io cannot be implemented in this case.

(ii) $\mu=\left(-\frac{1}{2}, \frac{I}{2}, \frac{I}{2}, \frac{I}{2}, I\right)$. Set $S^{\prime}=\{4\}$. Here the intersection form is not defined on all of $\mathrm{H}_{\varphi\left(\mathbf{S}^{\prime}\right)}^{1}\left(\mathrm{P}_{0}, \mathrm{~L}_{0}\right)$ but rather on $\mathrm{H}^{\prime}=\mathrm{H}_{c}^{1}\left(\mathrm{P}-\left\{x_{0}, x_{1}, x_{2}, x_{3}\right\}, \mathrm{L}^{\prime}\right)$ and $\mathrm{L}^{\prime}$ has monodromy corresponding to $\left(\frac{\mathrm{I}}{2}, \frac{\mathrm{I}}{2}, \frac{\mathrm{I}}{2}, \frac{\mathrm{I}}{2}\right)$. By $(2.2 \mathrm{I})$, the intersection form has signature $(I, I)$.

As in the euclidean case (13.2), the image of $\widetilde{w}_{\mu}: \widetilde{Q} \rightarrow V=H_{\varphi\left(\mathbf{S}^{\prime}\right)}^{1}\left(\mathbf{P}_{0}, \mathrm{~L}_{0}\right)$ lies on a two-dimensional affine hyperplane $\mathrm{H}$ which is parallel to $\mathrm{H}^{\prime}, \mathrm{H} \approx \mathrm{P}^{2}-\ell$, where $\ell$ is the line in the projective 2-space, which is defined by the vector subspace $\mathrm{H}^{\prime}$ of $\mathrm{V}$. As in the preceding case, $\widetilde{w}_{\mu}\left(\widetilde{Q}_{0 i}\right)$ lies in the line of fixed points of the pseudo-reflection $\gamma_{0 i}$ for $\mathrm{I} \leq i \leq 3$. By contrast, $\widetilde{w}_{\mu}\left(\widetilde{Q}_{i j}\right)$ is the point on the line $\ell$ fixed by the pseudoreflection $\gamma_{i j}$ for $\mathrm{I} \leq i \neq j \leq 3$. The monodromy group $\Gamma_{\alpha}^{\prime}$ is a matrix group with coefficients Gaussian integers and is therefore discrete.

\section{BIBLIOGRAPHY}

[1] Appel, P., a) CR Acad. Sci., Paris, I6 février ı880; b) Sur les fonctions hygergéométriques de deux variables, $J$. de Math., $3^{\text {e }}$ ser., VIII (1882), 173-216.

[2] BorEL, A., a) Density properties for certain subgroups of semi-simple groups without compact components, Ann. of Math., 72 ( 1960 ), I 79-188; b) Reduction theory for arithmetic groups, Proc. Symposia in Pure Math., IX (rg66), 20-25.

[3] Bores, A.-Harish Chandra, Arithmetic subgroups of algebraic groups, Ann. of Math., 75 (1962), 485-535

[4] Bourbaki, N., Groupes et Algebres de Lie, chap. V, Paris, Herman, 1968.

[5] Euler, L., “ Specimen transformationis singularis serierum", Sept. 3, r 778, Nova Acta Petropolitana, XII (I80I), 58-78. 
[6] Fricke, R., and KleIN, F., Vorlesungen über die Theorie der Automorphen Functionen, Bd. I, Leipzig, Teubner, 1897 . [7] Fox, R. H., Covering spaces with singularities, in Lefschetz Symposium, Princeton Univ. Press (1957), 243-262.

[8] Fuchs, L., Zur Theorie der linearen Differential gleichungen mit verändlerichen Coeffizienten, $J$. r. und angew. Math., 66 (1866), 12 I-16o.

[9] Hermite, C., Sur quelques équations différentielles linéaires, J. r. und angew. Math., 79 (1875), I I I-158.

[10] Hochschild, G. P., The Structure of Lie Groups, Holden-Day, San Francisco, 1965.

[11] KNeser, M., Strong approximation, Proc. of Symposia in Pure Math., IX (1966), 187-196.

[12] Lauriarlla, Sulle funzioni ipergeometriche a piu variabili, Rend. di Palermo, VII (1893), i 11-158.

[13] Le Vavasseur, R., Sur le système d'équations aux dérivées partielles simultanées auxquelles satisfait la série hypergéométrique à deux variables, J. Fac. Sci. Toulouse, VII (1896), 1-205.

[14] Mostow, G. D., a) Existence of nonarithmetic monodromy groups, Proc. Nat. Acad. Sci., 78 (1981), 5948-5950; b) Generalized Picard lattices arising from half-integral conditions, Publ. Math. I.H.E.S., this volume, 91-Io6.

[15] Mumford, D., Geometric Invariant Theory, Berlin, Springer, I965.

[16] Picard, E., a) Sur une extension aux fonctions de deux variables du problème de Riemann relatif aux fonctions hypergéométriques, Ann. ENS, 10 ( $188 \mathrm{r}$ ), 305-322; b) Sur les fonctions hyperfuchsiennes provenant des séries hypergéométriques de deux variables, Ann. ENS, III, 2 (1885), 357-384; c) Id., Bull. Soc. Math. Fr., $15(1887), 1^{8-1} 5^{2}$.

[17] Pochнамmer, L., Ueber hypergeometrische Functionen höherer Ordnung, J. r. und angew. Math., 71 (1870), $3^{16-362 .}$

[18] Riemann, B., Beiträge zur Theorie der durch die Gauss'sche Reihe $F(\alpha, \beta, \gamma, x)$ darstellbaren Functionen, Abh. Kon. Ges. d. Wiss zu Göttingen, VII (1857), Math. Classe, A-22.

[19] Sahapli, Ueber die Gauss'sche hypergeometrische Reihe, Math. Ann., III (1871), $286-295$.

[20] Scłrwarz, H. A., Ueber diejenigen Fälle in welchen die Gauss'sche hypergeometrische Reihe eine algebraisches Function ihres vierten Elementes darstellt, J. r. und angew. Math., 75 (1873), 292-335.

[21] TAkeuchi, K., Commensurability classes of arithmetic discrete triangle groups, J. Fac. Sci. Univ. Tokyo, 24 (1977), $201-212$.

[22] Terada, T., Problème de Riemann et fonctions automorphes provenant des fonctions hypergéometriques de plusieurs variables, J. Math. Kyoto Univ., 13 (1973), 557-578.

[23] TrTs, J., Classification of algebraic semi-simple groups, Proc. of Symposia in Pure Math., IX (1966), 33-62.

[24] Whitraker, E. T., and Watson, G. N., A course in modern analysis, Cambridge, University Press, ig62.

[25] Zucker, S., Hodge theory with degenerating coefficients, I, Ann. of Math., 109 (1979), 415-476.

Institute for Advanced Study,

Princeton, N.J., $0854^{\circ}$

Department of Mathematics,

Yale University,

Box 2155, Yale Station,

New Haven, CT, o6520

Manuscrit reçu le 2 juin 1983. 